\title{
ROBIN FUNCTIONS FOR COMPLEX MANIFOLDS AND APPLICATIONS1
}

\section{Kang-Tae Kim, Norman Levenberg and Hiroshi Yamaguchi}

\section{Introduction}

In [20] and [9] we analyzed the second variation of the Robin function associated to a smooth variation of domains in $\mathbb{C}^{n}$ for $n \geq 2$; i.e., $\mathcal{D}=$ $\cup_{t \in B}(t, D(t)) \subset B \times \mathbb{C}^{n}$ is a variation of domains $D(t)$ in $\mathbb{C}^{n}$ each containing a fixed point $z_{0}$ and with $\partial D(t)$ of class $C^{\infty}$ for $t \in B:=\{t \in \mathbb{C}:|t|<\rho\}$. For such $t$ and for $z \in \overline{D(t)}$ we let $g(t, z)$ be the $\mathbb{R}^{2 n}$-Green function for the domain $D(t)$ with pole at $z_{0}$; i.e., $g(t, z)$ is harmonic in $D(t) \backslash\left\{z_{0}\right\}, g(t, z)=0$ for $z \in \partial D(t)$, and $g(t, z)-\frac{1}{\left\|z-z_{0}\right\|^{2 n-2}}$ is harmonic near $z_{0}$. We call

$$
\lambda(t):=\lim _{z \rightarrow z_{0}}\left[g(t, z)-\frac{1}{\left\|z-z_{0}\right\|^{2 n-2}}\right]
$$

the Robin constant for $\left(D(t), z_{0}\right)$. Then

$$
\frac{\partial^{2} \lambda}{\partial t \partial \bar{t}}(t)=-c_{n} \int_{\partial D(t)} k_{2}(t, z)\left\|\nabla_{z} g\right\|^{2} d S_{z}-4 c_{n} \int_{D(t)} \sum_{a=1}^{n}\left|\frac{\partial^{2} g}{\partial t \partial \bar{z}_{a}}\right|^{2} d V_{z} .
$$

Here, $c_{n}=\frac{1}{(n-1) \Omega_{n}}$ is a positive dimensional constant where $\Omega_{n}$ is the area of the unit sphere in $\mathbb{C}^{n}, d S_{z}$ and $d V_{z}$ are the Euclidean area element on $\partial D(t)$ and volume element on $D(t), \nabla_{z} g=\left(\frac{\partial g}{\partial z_{1}}, \cdots, \frac{\partial g}{\partial z_{n}}\right)$ and

$$
k_{2}(t, z):=\left\|\nabla_{z} \psi\right\|^{-3}\left[\frac{\partial^{2} \psi}{\partial t \partial \bar{t}}\left\|\nabla_{z} \psi\right\|^{2}-2 \Re\left\{\frac{\partial \psi}{\partial t} \sum_{a=1}^{n} \frac{\partial \psi}{\partial \bar{z}_{a}} \frac{\partial^{2} \psi}{\partial \bar{t} \partial z_{a}}\right\}+\left|\frac{\partial \psi}{\partial t}\right|^{2} \Delta_{z} \psi\right]
$$

is the so-called Levi-curvature of $\partial \mathcal{D}$ at $(t, z)$. The function $\psi(t, z)$ is a defining function for $\mathcal{D}$ and the numerator is the sum of the Levi-form of $\psi$ applied to the $n$ complex tangent vectors $\left(-\frac{\partial \psi}{\partial z_{j}}, 0, \ldots, \frac{\partial \psi}{\partial t}, 0, \ldots, 0\right)$. In particular, if $\mathcal{D}$ is pseudoconvex (strictly pseudoconvex) at a point $(t, z)$ with $z \in \partial D(t)$, it follows that $k_{2}(t, z) \geq 0\left(k_{2}(t, z)>0\right)$ so that $-\lambda(t)$ is subharmonic (strictly subharmonic) in $B$. Given a bounded domain $D$ in $\mathbb{C}^{n}$, we let $\Lambda(z)$ be the Robin constant for $(D, z)$. If we fix a point $\zeta_{0} \in D$, for $\rho>0$ sufficiently small and $a \in \mathbb{C}^{n}$, the disk $\zeta_{0}+a B:=\left\{\zeta=\zeta_{0}+a t,|t|<\rho\right\}$ is contained in $D$. Using the biholomorphic mapping $T(t, z)=(t, z-a t)$ of

\footnotetext{
${ }^{1}$ This paper is dedicated to Professor John Wermer on the occasion of his 80th birthday.
} 
$B \times \mathbb{C}^{n}$, we get the variation of domains $\mathcal{D}=T(B \times D)$ where each domain $D(t):=T(t, D)=D-a t$ contains $\zeta_{0}$. Letting $\lambda(t)=\Lambda\left(\zeta_{0}+a t\right)$ denote the Robin constant for $\left(D(t), \zeta_{0}\right)$ and using (1.1) yields part of the following surprising result (cf., [20] and [9]).

Theorem 1.1. Let $D$ be a bounded pseudoconvex domain in $\mathbb{C}^{n}$ with $C^{\infty}$ boundary. Then $\log (-\Lambda(z))$ and $-\Lambda(z)$ are real-analytic, strictly plurisubharmonic exhaustion functions for $D$.

We now study a generalization of the second variation formula (1.1) to complex manifolds $M$ equipped with a Hermitian metric $d s^{2}$ and a smooth, nonnegative function $c$. Our purpose is that, with this added flexibility, we are able to give a criterion for a bounded, smoothly bounded, pseudoconvex domain D in a complex homogeneous space to be Stein. In particular, we are able to do the following:

1. Describe concretely all the non-Stein pseudoconvex domains $D$ in the complex torus of Grauert (section 5).

2. Give a description of all the non-Stein pseudoconvex domains $D$ in the special Hopf manifolds $\mathbb{H}_{n}$ (section 6).

3. Give a description of all the non-Stein pseudoconvex domains $D$ in the complex flag spaces $\mathcal{F}_{n}$ (section 7 ).

4. Give another explanation as to why all pseudoconvex subdomains of complex projective space, or, more generally, of complex Grassmannian manifolds, are Stein (Appendix A).

The metric $d s^{2}$ and the function $c$ give rise to a $c$-Green function and $c$-Robin constant associated to an open set $D \subset M$ and a point $p_{0} \in D$. We then take a variation $\mathcal{D}=\cup_{t \in B}(t, D(t)) \subset B \times M$ of domains $D(t)$ in $M$ each containing a fixed point $p_{0}$ and define a $c$-Robin function $\lambda(t)$. The precise definitions of these notions and the new variation formula (2.3) will be given in the next section. In section 3 we impose a natural condition (see (3.1)) on the metric $d s^{2}$ which will be useful for applications. Kähler metrics, in particular, satisfy (3.1). After discussing conditions which insure that the function $-\lambda$ is subharmonic, we will use (2.3) to develop a "rigidity lemma" (Lemma 4.1) which will imply, if $-\lambda$ is not strictly subharmonic, the existence of a nonvanishing, holomorphic vector field on $M$ with certain properties (Corollary 4.2). This will be a key tool in constructing strictly plurisubharmonic exhaustion functions for pseudoconvex subdomains $D$ with smooth boundary 
in certain complex Lie groups and in certain complex homogeneous spaces; i.e., we use these $c$-Robin functions to verify that $D$ is Stein.

Specifically, in section 5 we study pseudoconvex domains $D$ in a complex Lie group $M$. In a sense which will be made precise in Corollary 5.2 the functions $-\Lambda$ we construct in this setting are the "best possible" plurisubharmonic exhaustion functions: if a c-Robin function $\Lambda$ for $D$ is such that $-\Lambda$ is not strictly plurisubharmonic, then $D$ does not admit a strictly plurisubharmonic exhaustion function. We characterize the smoothly bounded, relatively compact pseudoconvex domains $D$ in a complex Lie group $M$ which are Stein in Theorem 5.1. Then we apply this result to describe all of the non-Stein pseudoconvex domains $D$ in the complex torus example of Grauert.

In section 6 we let $M$ be an $n$-dimensional complex homogeneous space with an associated connected complex Lie group $G \subset$ Aut $M$ of complex dimension $m \geq n$. We set up our $c$-Robin function machinery to discuss sufficient conditions on the pair $(M, G)$ such that for every smoothly bounded, relatively compact pseudoconvex domain $D$ in $M$, the function $-\lambda$ is strictly plurisubharmonic on $D$ (Theorem 6.2). In particular, the Grassmann manifolds $M=G(k, n)$ with $G=G L(n, \mathbb{C})$ satisfy one of these conditions.

In Theorems 6.3 and 6.4 we give an analogue of Theorem 5.1 to characterize the smoothly bounded, relatively compact pseudoconvex domains $D$ in a complex homogeneous space $M$ which are Stein. We immediately apply this result to special Hopf manifolds $\mathbb{H}_{n}$. Then in section 7 we apply the result to describe all of the non-Stein pseudoconvex domains $D$ in complex flag spaces $\mathcal{F}_{n}$ (Theorem [7.1).

Some of the results in this paper were announced without proof in [11] and [10]; in this paper, we provide complete proofs and illustrate the significance of this generalization of the second variation formula with applications and concrete examples. The material presented is completely self-contained; in particular, all concepts pertaining to Lie theory and homogeneous spaces are explained.

We thank Professor T. Ueda for his helpful advice in our study of Levi problems for flag spaces. We also thank Professor T. Morimoto for his useful comments regarding Lie algebras. 


\section{The variation formula.}

Our general set-up is this: let $M$ be an $n$-dimensional complex manifold (compact or not) equipped with a Hermitian metric

$$
d s^{2}=\sum_{a, b=1}^{n} g_{a \bar{b}} d z_{a} \otimes d \bar{z}_{b}
$$

and let $\omega:=i \sum_{a, b=1}^{n} g_{a \bar{b}} d z_{a} \wedge d \bar{z}_{b}$ be the associated real $(1,1)$ form. As in the introduction, we take $n \geq 2$. We write $g^{\bar{a} b}:=\left(g_{a \bar{b}}\right)^{-1}$ for the elements of the inverse matrix to $\left(g_{a \bar{b}}\right)$ and $G:=\operatorname{det}\left(g_{a \bar{b}}\right)$. Note that $\omega^{n}=2^{n} n ! G d x_{1} \wedge$ $\cdots \wedge d x_{2 n}$ locally where $z_{k}=x_{2 k-1}+i x_{2 k}$. For a domain $W \subset M$, we let $\mathcal{L}^{p, q}(W)$ denote the $(p, q)$ forms on $W$ with complex-valued, $C^{\infty}(W)$ functions as coefficients. We have the standard linear operators

$$
\begin{gathered}
*: \mathcal{L}^{p, q}(W) \rightarrow \mathcal{L}^{n-q, n-p}(W), \\
\partial: \mathcal{L}^{p, q}(W) \rightarrow \mathcal{L}^{p+1, q}(W), \\
\bar{\partial}: \mathcal{L}^{p, q}(W) \rightarrow \mathcal{L}^{p, q+1}(W), \\
\delta:=-* \partial *: \mathcal{L}^{p, q}(W) \rightarrow \mathcal{L}^{p, q-1}(W), \\
\bar{\delta}:=-* \bar{\partial} *: \mathcal{L}^{p, q}(W) \rightarrow \mathcal{L}^{p-1, q}(W),
\end{gathered}
$$

and $d=\partial+\bar{\partial}$. We get the box Laplacian operator

$$
\delta \bar{\partial}+\bar{\partial} \delta: \mathcal{L}^{p, q}(W) \rightarrow \mathcal{L}^{p, q}(W)
$$

and its conjugate

$$
\bar{\delta} \partial+\partial \bar{\delta}: \mathcal{L}^{p, q}(W) \rightarrow \mathcal{L}^{p, q}(W) .
$$

Adding these, we obtain the Laplacian operator

$$
\Delta=\delta \bar{\partial}+\bar{\partial} \delta+\bar{\delta} \partial+\partial \bar{\delta}
$$

which is a real operator; in local coordinates acting on functions this has the form

$$
\begin{aligned}
\Delta u & =-2\left[\sum_{a, b=1}^{n} g^{\bar{b} a} \frac{\partial^{2} u}{\partial \bar{z}_{b} \partial z_{a}}+\frac{1}{2} \sum_{a, b=1}^{n}\left(\frac{1}{G} \frac{\partial\left(G g^{\bar{b} a}\right)}{\partial z_{a}} \frac{\partial u}{\partial \bar{z}_{b}}+\frac{1}{G} \frac{\partial\left(G g^{\bar{a} b}\right)}{\partial \bar{z}_{a}} \frac{\partial u}{\partial z_{b}}\right)\right] \\
& =:-2[P u+R u] .
\end{aligned}
$$


We call $P u$ the principal part of $\Delta u$. Also, we remark that if $d s^{2}$ is Kähler, i.e., if $d \omega=0$, then $\Delta u=-2 P u=-2 \sum_{a, b=1}^{n} g^{\bar{b} a} \frac{\partial^{2} u}{\partial \bar{z}_{b} \partial z_{a}}$. As usual, we set, for any $\alpha \in \mathcal{L}^{p, q}(W),\|\alpha\|_{W}^{2}=\int_{W} \alpha \wedge \overline{* \alpha} \geq 0$.

Given a nonnegative $C^{\infty}$ function $c=c(z)$ on $M$, we call a $C^{\infty}$ function $u$ on an open set $D \subset M c$-harmonic on $D$ if $\Delta u+c u=0$ on $D$. Choosing local coordinates near a fixed point $p_{0} \in M$ and a coordinate neighborhood $U$ of $p_{0}$ such that $\left[g_{a \bar{b}}\left(p_{0}\right)\right]_{a, b=1, \ldots, n}=\left[\delta_{a b}\right]_{a, b=1, \ldots, n}$, the Laplacian $\Delta$ corresponds to a second-order elliptic operator $\Delta$ in $\mathbb{C}^{n}$. In particular, we can find a $c$-harmonic function $Q_{0}$ in $U \backslash\left\{p_{0}\right\}$ satisfying

$$
\lim _{p \rightarrow p_{0}} Q_{0}(p) d\left(p, p_{0}\right)^{2 n-2}=1
$$

where $d\left(p, p_{0}\right)$ is the geodesic distance between $p$ and $p_{0}$ with respect to the metric $d s^{2}$. We call $Q_{0}$ a fundamental solution for $\Delta$ and $c$ at $p_{0}$. Fixing $p_{0}$ in a smoothly bounded domain $D \Subset M$ and fixing a fundamental solution $Q_{0}$, the $c$-Green function $g$ for $\left(D, p_{0}\right)$ is the $c$-harmonic function in $D \backslash\left\{p_{0}\right\}$ satisfying $g=0$ on $\partial D(g$ is continuous up to $\partial D)$ with $g(p)-Q_{0}(p)$ regular at $p_{0}$. The $c$-Green function always and uniquely exists (cf., [13]) and is nonnegative on $D$. Then

$$
\lambda:=\lim _{p \rightarrow p_{0}}\left[g(p)-Q_{0}(p)\right]
$$

is called the $c$-Robin constant for $\left(D, p_{0}\right)$. Thus we have

$$
g(p)=Q_{0}(p)+\lambda+h(p)
$$

for $p$ near $p_{0}$, where $h\left(p_{0}\right)=0$. In case $M$ is compact, if $c \not \equiv 0$ on $M$, then the $c$-Green function $g$ for $\left(M, p_{0}\right)$ exists and is positive on $M$, hence the $c$-Robin constant is finite. But if $c \equiv 0$ on $M$, a $c$-harmonic function is harmonic and cannot attain its minimum; thus, in this case, $g(z) \equiv+\infty$ on $M$ (cf., [13]). In this case we set $\lambda=+\infty$.

Now let $\mathcal{D}=\cup_{t \in B}(t, D(t)) \subset B \times M$ be a $C^{\infty}$ variation of domains $D(t)$ in $M$ each containing a fixed point $p_{0}$ and with $\partial D(t)$ of class $C^{\infty}$ for $t \in B$. This means that there exists $\psi(t, z)$ which is $C^{\infty}$ in a neighborhood $N \subset B \times M$ of $\{(t, z): t \in B, z \in \partial D(t)\}$, negative in $N \cap\{(t, z): t \in B, z \in D(t)\}$, and for each $t \in B, z \in \partial D(t)$, we require that $\psi(t, z)=0$ and $\frac{\partial \psi}{\partial z_{i}}(t, z) \neq 0$ for some $i=1, \ldots, n$. We call $\psi(t, z)$ a defining function for $\mathcal{D}$. Assume that $B \times\left\{p_{0}\right\} \subset \mathcal{D}$. Let $g(t, z)$ be the $c$-Green function for $\left(D(t), p_{0}\right)$ and $\lambda(t)$ the corresponding $c$-Robin constant. The hypothesis that $\mathcal{D}$ be a $C^{\infty}$ variation implies that for each $t \in B$, the $c$-Green function $g(t, z)$ extends of class $C^{\infty}$ beyond $\partial D(t)$; this follows from the general theory of partial differential 
equations. Most of the calculations and the subsequent results in this paper remain valid under weaker $\left(C^{2}\right.$ or $\left.C^{3}\right)$ regularity assumptions on $\mathcal{D}$.

Our formulas are the following:

$$
\begin{aligned}
& \frac{\partial \lambda}{\partial t}(t)=-c_{n} \int_{\partial D(t)} k_{1}(t, z) \sum_{a, b=1}^{n}\left(g^{\bar{a} b} \frac{\partial g}{\partial \bar{z}_{a}} \frac{\partial g}{\partial z_{b}}\right) d \sigma_{z} \\
& \frac{\partial^{2} \lambda}{\partial t \partial \bar{t}}(t)=-c_{n} \int_{\partial D(t)} k_{2}(t, z) \sum_{a, b=1}^{n}\left(g^{\bar{a} b} \frac{\partial g}{\partial \bar{z}_{a}} \frac{\partial g}{\partial z_{b}}\right) d \sigma_{z} \\
& \quad-\frac{c_{n}}{2^{n-2}}\left\{\left\|\bar{\partial} \frac{\partial g}{\partial t}\right\|_{D(t)}^{2}+\frac{1}{2}\left\|\sqrt{c} \frac{\partial g}{\partial t}\right\|_{D(t)}^{2}+\frac{1}{2} \Re \int_{D(0)} \frac{\partial g}{\partial t}\left[\frac{1}{i} \partial * \omega \wedge \bar{\partial} \frac{\partial g}{\partial \bar{t}}+\frac{1}{i} \bar{\partial} * \omega \wedge \partial \frac{\partial g}{\partial \bar{t}}\right]\right\} \\
& \quad:=-c_{n} I-\frac{c_{n}}{2^{n-2}} J
\end{aligned}
$$

where $d \sigma_{z}$ is the area element on $\partial D(t)$ with respect to the Hermitian metric, $c_{n}=\frac{1}{(n-1) \Omega_{n}}$ and

$$
\begin{aligned}
& k_{1}(t, z):=\left[\sum_{a, b=1}^{n} g^{\bar{a} b} \frac{\partial \psi}{\partial \bar{z}_{a}} \frac{\partial \psi}{\partial z_{b}}\right]^{-1 / 2} \frac{\partial \psi}{\partial t}, \\
& k_{2}(t, z):=\left[\sum_{a, b=1}^{n} g^{\bar{a} b} \frac{\partial \psi}{\partial \bar{z}_{a}} \frac{\partial \psi}{\partial z_{b}}\right]^{-3 / 2} \times \\
& {\left[\frac{\partial^{2} \psi}{\partial t \partial \bar{t}}\left(\sum_{a, b=1}^{n} g^{\bar{a} b} \frac{\partial \psi}{\partial \bar{z}_{a}} \frac{\partial \psi}{\partial z_{b}}\right)-2 \Re\left\{\frac{\partial \psi}{\partial t}\left(\sum_{a, b=1}^{n} g^{\bar{a} b} \frac{\partial \psi}{\partial \bar{z}_{a}} \frac{\partial^{2} \psi}{\partial z_{b} \partial \bar{t}}\right)\right\}-\frac{1}{2}\left|\frac{\partial \psi}{\partial t}\right|^{2} \Delta_{z} \psi\right],}
\end{aligned}
$$

$\psi(t, z)$ being a defining function for $\mathcal{D}$. Here, $k_{i}(t, z)(i=1,2)$ is a real-valued function for $(t, z) \in \partial \mathcal{D}$ which is independent of both the choice of defining function for $\mathcal{D}$ and of the choice of local parameter $z$ in the manifold $M$. We call $k_{2}(t, z)$ the Levi scalar curvature with respect to the metric $d s^{2}$.

Formula (2.2) is a generalization of the classical Hadamard variation formula. For the study of several complex variables the variation formula (2.3) of the second order is fundamental and we now give the proof. First, for each $t \in B$, the equation

$$
g(t, p)=Q_{0}(p)+\lambda(t)+h(t, p),
$$

where $h\left(t, p_{0}\right)=0$ for all $t \in B$, holds; hence we have, for $p \neq p_{0}$,

$$
\frac{\partial^{2} g}{\partial t \partial \bar{t}}(t, p)=\frac{\partial^{2} \lambda}{\partial t \partial \bar{t}}(t)+\frac{\partial^{2} h}{\partial t \partial \bar{t}}(t, p) .
$$


Since $\frac{\partial^{2} h}{\partial t \partial \bar{t}}\left(t, p_{0}\right)=0$ for all $t \in B$, if we set $\frac{\partial^{2} g}{\partial t \partial \bar{t}}\left(t, p_{0}\right)=\frac{\partial^{2} \lambda}{\partial t \partial \bar{t}}(t)$, it follows that $\frac{\partial^{2} g}{\partial t \partial \bar{t}}(t, p)$ is a $c$-harmonic function on all of $D(t)$ even though $g(t, p)$ has a singularity at $p_{0}$. Fix $t_{0} \in B$. Using Green's formula for $c$-harmonic functions, we thus obtain the formula

$$
\frac{\partial^{2} \lambda}{\partial t \partial \bar{t}}\left(t_{0}\right)=\frac{-c_{n}}{2^{n}} \int_{\partial D\left(t_{0}\right)} \frac{\partial^{2} g}{\partial t \partial \bar{t}}\left(t_{0}, z\right) * d g\left(t_{0}, z\right) .
$$

We note that, in $\mathbb{C}^{n}$, from the definition of the $*$-operator, we have

$$
* d g\left(t_{0}, z\right)=-2^{n}\left\|\nabla_{z} g\left(t_{0}, z\right)\right\| d S_{z}=2^{n-1} \frac{\partial g}{\partial n_{z}}\left(t_{0}, z\right) d S_{z}
$$

for $z \in \partial D\left(t_{0}\right)$, where $d S_{z}$ and $n_{z}$ are the Euclidean area element and the unit outer normal vector for $\partial D\left(t_{0}\right)$ at $z$.

We may assume $t_{0}=0$. Now using the fact that $-g(t, z)$ is a defining function for $\mathcal{D}$, we can write, from (2.4),

$$
\begin{aligned}
& k_{2}(0, z) \cdot\left(\sum_{a, b=1}^{n} g^{\bar{a} b} \frac{\partial g}{\partial \bar{z}_{a}} \frac{\partial g}{\partial z_{b}}\right)^{3 / 2}=-\frac{\partial^{2} g}{\partial t \partial \bar{t}}\left(\sum_{a, b=1}^{n} g^{\bar{a} b} \frac{\partial g}{\partial \bar{z}_{a}} \frac{\partial g}{\partial z_{b}}\right) \\
& +2 \Re\left[\left(\sum_{a, b=1}^{n} g^{\bar{a} b} \frac{\partial^{2} g}{\partial z_{b} \partial \bar{t}} \frac{\partial g}{\partial \bar{z}_{a}}\right) \frac{\partial g}{\partial t}\right]+\frac{1}{2}\left|\frac{\partial g}{\partial t}\right|^{2} \Delta g .
\end{aligned}
$$

Since $\Delta g+c g=0$ on $\partial D(0)$ (here we use the fact that $g$ is of class $C^{\infty}$ on $\left.\overline{D(0)} \backslash\left\{p_{0}\right\}\right)$ and $g=0$ on $\partial D(0)$, we have from (2.5) and (2.6)

$$
\begin{aligned}
& \frac{\partial^{2} \lambda}{\partial t \partial \bar{t}}(0)=\frac{-c_{n}}{2^{n}} \int_{\partial D(0)}\left\{-k_{2}(0, z) \sum_{a, b=1}^{n}\left(g^{\bar{a} b} \frac{\partial g}{\partial \bar{z}_{a}} \frac{\partial g}{\partial z_{b}}\right)^{1 / 2}\right. \\
& \left.+\frac{2 \Re \sum_{a, b=1}^{n}\left(g^{\bar{a} b} \frac{\partial^{2} g}{\partial z_{b} \partial \bar{t}} \frac{\partial g}{\partial \bar{z}_{a}}\right) \frac{\partial g}{\partial t}}{\sum_{a, b=1}^{n} g^{\bar{a} b} \frac{\partial g}{\partial \bar{z}_{a}} \frac{\partial g}{\partial z_{b}}}\right\} * d g(0, z) \\
& \equiv(I)+(I I) \text {. }
\end{aligned}
$$

In general, for any $C^{\infty}$ defining function $v$ on $\overline{D(0)}(v=0$ on $\partial D(0)$ and $v<0$ on $D(0))$,

$$
* d v=2^{n}\left(\sum_{a, b=1}^{n} g^{\bar{a} b} \frac{\partial v}{\partial \bar{z}_{a}} \frac{\partial v}{\partial z_{b}}\right)^{1 / 2} d \sigma_{z} \geq 0
$$


for $z \in \partial D(0)$, where $d \sigma_{z}$ is the area element on $\partial D(0)$ at $z$ with respect to the metric $d s^{2}$. We apply this to $v=-g(0, z)$, plug this into $(I)$, and obtain the formula

$$
(I)=-c_{n} \int_{\partial D(0)} k_{2}(0, z) \sum_{a, b=1}^{n}\left(g^{\bar{a} b} \frac{\partial g}{\partial \bar{z}_{a}} \frac{\partial g}{\partial z_{b}}\right) d \sigma_{z}
$$

Now we work with $(I I)$. We need to calculate

$$
\frac{\partial g}{\partial \bar{z}_{a}} * d g(0, z)
$$

on $\partial D(0)$. To this end, we first note that for a function $u$, we have the following formulas for $* \partial u$ and $* \bar{\partial} u$ :

$$
\begin{gathered}
* \partial u=-i^{n} \sum_{a, b=1}^{n} G g^{\bar{a} b} \frac{\partial u}{\partial z_{b}} d z_{a} \wedge d z_{1} \wedge d \bar{z}_{1} \wedge \cdots d \widehat{d z_{a} \wedge d \bar{z}_{a}} \cdots \wedge d z_{n} \wedge d \bar{z}_{n} \\
* \bar{\partial} u=i^{n} \sum_{a, b=1}^{n} G g^{\bar{b} a} \frac{\partial u}{\partial \bar{z}_{b}} d \bar{z}_{a} \wedge d z_{1} \wedge d \bar{z}_{1} \wedge \cdots d \widehat{z_{a} \wedge d \bar{z}_{a}} \cdots \wedge d z_{n} \wedge d \bar{z}_{n} .
\end{gathered}
$$

Now if $u=0$ on $\partial D(0), d u=\partial u+\bar{\partial} u=0$ along $\partial D(0)$ and we obtain

$$
* d u=-2 i^{n} \sum_{a, b=1}^{n} G g^{\bar{a} b} \frac{\partial u}{\partial z_{b}} d z_{a} \wedge d z_{1} \wedge d \bar{z}_{1} \wedge \cdots d \widehat{d z_{a} \wedge d \bar{z}_{a}} \cdots \wedge d z_{n} \wedge d \bar{z}_{n} .
$$

Applying this to $u=g(0, z)$ on $\partial D(0)$, we get

$\frac{\partial g}{\partial \bar{z}_{a}} * d g(0, z)=-2 i^{n} \sum_{i, j=1}^{n} G g^{i j} \frac{\partial g}{\partial \bar{z}_{a}} \frac{\partial g}{\partial z_{j}} d z_{i} \wedge d z_{1} \wedge d \bar{z}_{1} \wedge \cdots d \widehat{d z_{i} \wedge d \bar{z}_{i}} \cdots \wedge d z_{n} \wedge d \bar{z}_{n}$.

Again we use the fact that $g(0, z)=0$ on $\partial D(0)$ so that $d g(0, z)=0$ along $\partial D(0)$. Then, if $i \neq a, \frac{\partial g}{\partial \bar{z}_{a}} d \bar{z}_{a}$ in the above formula can be replaced by $-\frac{\partial g}{\partial \bar{z}_{i}} d \bar{z}_{i}$. It follows that

$\frac{\partial g}{\partial \bar{z}_{a}} * d g(0, z)=-2 i^{n}\left(G \sum_{i, j=1}^{n} g^{i j} \frac{\partial g}{\partial z_{j}} \frac{\partial g}{\partial \bar{z}_{i}}\right) d z_{a} \wedge d z_{1} \wedge d \bar{z}_{1} \wedge \cdots d \widehat{z_{a} \wedge d} \bar{z}_{a} \cdots \wedge d z_{n} \wedge d \bar{z}_{n}$

where the term in parenthesis is independent of $a=1, \ldots, n$. We plug this into $(I I)$ where

$$
(I I)=\frac{-c_{n}}{2^{n}} \int_{\partial D(0)} F
$$


and

$$
\begin{aligned}
& F:=\left\{\frac{2 \Re \sum_{a, b=1}^{n}\left(g^{\bar{a} b} \frac{\partial^{2} g}{\partial z_{b} \partial \bar{t}} \frac{\partial g}{\partial \bar{z}_{a}}\right) \frac{\partial g}{\partial t}}{\sum_{a, b=1}^{n} g^{\bar{a} b} \frac{\partial g}{\partial \bar{z}_{a}} \frac{\partial g}{\partial z_{b}}}\right\} * d g(0, z) \\
&=-4 \Re\left\{i^{n}\left(\sum_{a, b=1}^{n} G g^{\bar{a} b} \frac{\partial^{2} g}{\partial z_{b} \partial \bar{t}} \frac{\partial g}{\partial t}\right)\right. \\
&\left.\quad d z_{a} \wedge d z_{1} \wedge d \bar{z}_{1} \wedge \cdots d \widehat{z_{a} \wedge d \bar{z}_{a}} \cdots \wedge d z_{n} \wedge d \bar{z}_{n}\right\} .
\end{aligned}
$$

Note that the denominator cancels.

Next we use the relation

$$
* \partial\left(\frac{\partial g}{\partial \bar{t}}\right)=-i^{n} \sum_{a, b=1}^{n} G g^{\bar{a} b} \frac{\partial^{2} g}{\partial z_{b} \partial \bar{t}} d z_{a} \wedge d z_{1} \wedge d \bar{z}_{1} \wedge \cdots d \widehat{z_{a} \wedge d \bar{z}_{a}} \cdots \wedge d z_{n} \wedge d \bar{z}_{n}
$$

to obtain

$$
F=4 \Re\left\{\frac{\partial g}{\partial t}\left(* \partial\left(\frac{\partial g}{\partial \bar{t}}\right)\right)\right\}
$$

on $\partial D(0)$.

Thus we obtain

$$
\begin{aligned}
\frac{\partial^{2} \lambda}{\partial t \partial \bar{t}}(0)= & -c_{n} \int_{\partial D(0)} k_{2}(0, z)\left[\sum_{a, b=1}^{n} g^{\bar{a} b} \frac{\partial g}{\partial \bar{z}_{a}} \frac{\partial g}{\partial z_{b}}\right] d \sigma_{z} \\
& -\frac{c_{n}}{2^{n-2}} \Re \int_{\partial D(0)} \frac{\partial g}{\partial t}\left(* \partial\left(\frac{\partial g}{\partial \bar{t}}\right)\right)=:-I-J
\end{aligned}
$$

where

$$
J:=\frac{c_{n}}{2^{n-2}} \Re \int_{\partial D(0)} f
$$

and

$$
f:=\frac{\partial g}{\partial t}\left(* \partial\left(\frac{\partial g}{\partial \bar{t}}\right)\right)
$$

is an $(n, n-1)$ form. We want to convert $J$ into a volume integral; to do so we must compute $\bar{\partial} f$ since, $f$ being $(n, n-1)$ form,

$$
J=\frac{c_{n}}{2^{n-2}} \Re \int_{D(0)} d f=\frac{c_{n}}{2^{n-2}} \Re \int_{D(0)} \bar{\partial} f .
$$

We get two terms whose sum comprise $\bar{\partial} f$ :

$$
\text { (i) }=\left(\bar{\partial} \frac{\partial g}{\partial t}\right) \wedge\left(* \partial\left(\frac{\partial g}{\partial \bar{t}}\right)\right) ;
$$




$$
\text { (ii) }=\frac{\partial g}{\partial t} \bar{\partial}\left(* \partial\left(\frac{\partial g}{\partial \bar{t}}\right)\right) \text {. }
$$

Now

$$
\int_{D(0)}(\mathrm{i})=\int_{D(0)}\left(\bar{\partial} \frac{\partial g}{\partial t}\right) \wedge\left(* \partial\left(\frac{\partial g}{\partial \bar{t}}\right)\right)=\left\|\bar{\partial} \frac{\partial g}{\partial t}\right\|_{D(0)}^{2}
$$

We note that in $\mathbb{C}^{n}$, we have $\left\|\bar{\partial} \frac{\partial g}{\partial t}\right\|_{D(0)}^{2}=2^{n} \int_{D(0)}\left(\sum_{i=1}^{n}\left|\frac{\partial^{2} g}{\partial t \partial \bar{z}_{i}}\right|^{2}\right) d V_{z}$, where $d V_{z}$ is the Euclidean volume element of $\mathbb{C}^{n}$, so that the first term $\frac{c_{n}}{2^{n-2}} \Re \int_{D(0)}(\mathrm{i})$ of $J$ coincides with the last term of formula (1.1).

Next, for (ii),

$\bar{\partial}\left(* \partial\left(\frac{\partial g}{\partial \bar{t}}\right)\right)=i^{n} \sum_{a, b=1}^{n}\left[\frac{\partial\left(G g^{\bar{a} b}\right)}{\partial \bar{z}_{a}} \frac{\partial^{2} g}{\partial z_{b} \partial \bar{t}}+G g^{\bar{a} b} \frac{\partial^{3} g}{\partial \bar{z}_{a} \partial z_{b} \partial \bar{t}}\right] d z_{1} \wedge d \bar{z}_{1} \wedge \cdots \wedge d z_{n} \wedge d \bar{z}_{n}$.

Using the relation

$$
\partial * \omega=-i^{n+1} \sum_{a, b=1}^{n} \frac{\partial\left(G g^{\bar{a} b}\right)}{\partial z_{b}} d z_{1} \wedge d \bar{z}_{1} \wedge \cdots d z_{a} \wedge \widehat{d \bar{z}}_{a} \cdots \wedge d z_{n} \wedge d \bar{z}_{n},
$$

with an analogous formula for $\bar{\partial} * \omega$, if $u$ is a complex-valued function, we obtain

$$
(\bar{\partial} * \omega) \wedge \partial u=i^{n+1} \sum_{a, b=1}^{n} \frac{\partial\left(G g^{\bar{a} b}\right)}{\partial \bar{z}_{a}} \frac{\partial u}{\partial z_{b}} d z_{1} \wedge d \bar{z}_{1} \wedge \cdots \wedge d z_{n} \wedge d \bar{z}_{n} .
$$

Also

$$
\begin{aligned}
\partial * \bar{\partial} u & =i^{n} \sum_{a, b=1}^{n}\left[G g^{\bar{b} a} \frac{\partial^{2} u}{\partial z_{a} \partial \bar{z}_{b}}+\frac{\partial\left(G g^{\bar{b} a}\right)}{\partial z_{a}} \frac{\partial u}{\partial \bar{z}_{b}}\right] d z_{1} \wedge d \bar{z}_{1} \wedge \cdots \wedge d z_{n} \wedge d \bar{z}_{n} \\
& =: D_{2} u+D_{1} u
\end{aligned}
$$

Similarly,

$$
\bar{\partial} * \partial u=D_{2} u+\overline{D_{1} \bar{u}} .
$$

Finally, it is straightforward to verify that

$$
(\bar{\partial} * \omega) \wedge \partial u=i \overline{D_{1} \bar{u}} .
$$

Using these relations, we obtain

$$
(\bar{\partial} * \omega) \wedge \partial \frac{\partial g}{\partial \bar{t}}=i \overline{D_{1}\left(\frac{\partial g}{\partial t}\right)}
$$


and

$$
(\partial * \omega) \wedge \bar{\partial} \frac{\partial g}{\partial \bar{t}}=-i D_{1}\left(\frac{\partial g}{\partial \bar{t}}\right)
$$

Now since $g$ is a function, $\delta g=\bar{\delta} g=0$; thus we rewrite $\Delta g+c g=0$ as

$$
(\bar{\delta} \partial+\delta \bar{\partial}) g+c g=-(* \bar{\partial} * \partial+* \partial * \bar{\partial}) g+c g=0 .
$$

Applying $*$ to this relation and using $* * K=K$, we have

$$
-(\bar{\partial} * \partial+\partial * \bar{\partial}) g+* c g=0 .
$$

This last equation can be written using $D_{1}$ and $D_{2}$ as

$$
-\left(D_{2} g+\overline{D_{1} g}+D_{2} g+D_{1} g\right)+* c g=0 .
$$

Hence

$$
D_{2} g=\frac{-1}{2}\left[D_{1} g+\overline{D_{1} g}-* c g\right]
$$

Equation (2.8) is valid in $D(0) \backslash\left\{p_{0}\right\}$ - indeed, in each $D(t) \backslash\left\{p_{0}\right\}$ - as an equality of $(n, n)$ forms. Differentiating (2.8) with respect to $\bar{t}$, we thus obtain

$$
D_{2}\left(\frac{\partial g}{\partial \bar{t}}\right)=\frac{-1}{2}\left[D_{1}\left(\frac{\partial g}{\partial \bar{t}}\right)+\overline{D_{1}\left(\frac{\partial g}{\partial t}\right)}-* c \frac{\partial g}{\partial \bar{t}}\right]
$$

in $D(t)$.

We use (2.9) in (ii):

$$
\begin{gathered}
\bar{\partial}\left(* \partial\left(\frac{\partial g}{\partial \bar{t}}\right)\right)=D_{2}\left(\frac{\partial g}{\partial \bar{t}}\right)+\overline{D_{1}\left(\frac{\partial g}{\partial t}\right)}=\frac{-1}{2}\left[D_{1}\left(\frac{\partial g}{\partial \bar{t}}\right)-\overline{D_{1}\left(\frac{\partial g}{\partial t}\right)}-* c \frac{\partial g}{\partial \bar{t}}\right] \\
=\frac{-1}{2}\left[\frac{-1}{i} \partial * \omega \wedge \bar{\partial} \frac{\partial g}{\partial \bar{t}}-\frac{1}{i} \bar{\partial} * \omega \wedge \partial \frac{\partial g}{\partial \bar{t}}-* c \frac{\partial g}{\partial \bar{t}}\right]
\end{gathered}
$$

Inserting parts (i) and (ii) of $\bar{\partial} f$ back in to $J=\frac{c_{n}}{2^{n-2}} \Re \int_{D(0)} \bar{\partial} f$, we obtain $J=\frac{c_{n}}{2^{n-2}} \Re \int_{D(0)}\left\{\bar{\partial} \frac{\partial g}{\partial t} \wedge * \partial \frac{\partial g}{\partial \bar{t}}+\frac{1}{2} \frac{\partial g}{\partial t}\left[\frac{1}{i} \partial * \omega \wedge \bar{\partial} \frac{\partial g}{\partial \bar{t}}+\frac{1}{i} \bar{\partial} * \omega \wedge \partial \frac{\partial g}{\partial \bar{t}}+* c \frac{\partial g}{\partial \bar{t}}\right]\right\}$.

Hence,

$$
J=\frac{c_{n}}{2^{n-2}} \int_{D(0)}\left\{\bar{\partial} \frac{\partial g}{\partial t} \wedge * \partial \frac{\partial g}{\partial \bar{t}}+\Re\left(\frac{1}{2} \frac{\partial g}{\partial t}\left[\frac{1}{i} \partial * \omega \wedge \bar{\partial} \frac{\partial g}{\partial \bar{t}}+\frac{1}{i} \bar{\partial} * \omega \wedge \partial \frac{\partial g}{\partial \bar{t}}\right]\right)+\frac{1}{2} c\left|\frac{\partial g}{\partial t}\right|^{2} \frac{\omega^{n}}{n !}\right\} .
$$


Thus we obtain

$$
\begin{gathered}
\frac{\partial^{2} \lambda}{\partial t \partial \bar{t}}(0)=-c_{n} \int_{\partial D(0)} k_{2}(0, z) \sum_{a, b=1}^{n}\left(g^{\bar{a} b} \frac{\partial g}{\partial \bar{z}_{a}} \frac{\partial g}{\partial z_{b}}\right) d \sigma_{z} \\
-\frac{c_{n}}{2^{n-2}}\left\{\left\|\bar{\partial} \frac{\partial g}{\partial t}\right\|_{D(0)}^{2}+\frac{1}{2}\left\|\sqrt{c} \frac{\partial g}{\partial t}\right\|_{D(0)}^{2}+\frac{1}{2} \Re \int_{D(0)} \frac{\partial g}{\partial t}\left[\frac{1}{i} \partial * \omega \wedge \bar{\partial} \frac{\partial g}{\partial \bar{t}}+\frac{1}{i} \bar{\partial} * \omega \wedge \partial \frac{\partial g}{\partial \bar{t}}\right]\right\}
\end{gathered}
$$

which is (2.3) for $t=0$. The second order variation formula (2.3) is thus proved.

Remark 2.1. Note that (2.3) reduces to (1.1) if $M=\mathbb{C}^{n}, d s^{2}=|d z|^{2}$ is the Euclidean metric, and $c \equiv 0$.

\section{Subharmonicity of $-\lambda$.}

We impose the following condition on the Hemitian metric $d s^{2}$ on $M$ :

$$
\partial * \omega=0 \quad \text { on } M
$$

Remark 3.1. A Kähler metric $d s^{2}$ on $M$ (i.e., $d \omega=0$ ) satisfies (3.1). Indeed, since $* \omega=\frac{\omega^{n-1}}{n-1}$ on $M$ and $\omega$ is real, it follows that

$$
\partial * \omega=\partial \omega \wedge \omega^{n-2}=\frac{1}{2} d \omega \wedge \omega^{n-2}=0 \quad \text { on } M
$$

Theorem 3.1. Assume that $d s^{2}$ satisfies condition (3.1). Then:

1. The Levi scalar curvature $k_{2}(t, z)$ of $\partial \mathcal{D}$ reduces to:

$$
\begin{aligned}
& K_{2}(t, z):=\left[\sum_{a, b=1}^{n} g^{\bar{a} b} \frac{\partial \psi}{\partial \bar{z}_{a}} \frac{\partial \psi}{\partial z_{b}}\right]^{-3 / 2} \times \\
& {\left[\frac{\partial^{2} \psi}{\partial t \partial \bar{t}}\left(\sum_{a, b=1}^{n} g^{\bar{a} b} \frac{\partial \psi}{\partial \bar{z}_{a}} \frac{\partial \psi}{\partial z_{b}}\right)-2 \Re\left\{\frac{\partial \psi}{\partial t}\left(\sum_{a, b=1}^{n} g^{\bar{a} b} \frac{\partial \psi}{\partial \bar{z}_{a}} \frac{\partial^{2} \psi}{\partial z_{b} \partial \bar{t}}\right)\right\}+\left|\frac{\partial \psi}{\partial t}\right|^{2}\left(\sum_{a, b=1}^{n} g^{\bar{a} b} \frac{\partial^{2} \psi}{\partial \bar{z}_{a} \partial z_{b}}\right)\right] .}
\end{aligned}
$$

2. The second variation formula (2.3) of $\lambda(t)$ reduces to:

$$
\begin{aligned}
\frac{\partial^{2} \lambda}{\partial t \partial \bar{t}}(t)=-c_{n} \int_{\partial D(t)} & K_{2}(t, z) \sum_{a, b=1}^{n}\left(g^{\bar{a} b} \frac{\partial g}{\partial \bar{z}_{a}} \frac{\partial g}{\partial z_{b}}\right) d \sigma_{z} \\
- & \frac{c_{n}}{2^{n-2}}\left\{\left\|\bar{\partial} \frac{\partial g}{\partial t}\right\|_{D(t)}^{2}+\frac{1}{2}\left\|\sqrt{c} \frac{\partial g}{\partial t}\right\|_{D(t)}^{2}\right\}
\end{aligned}
$$


Proof. In local coordinates $z=\left(z_{1}, \ldots, z_{n}\right)$ we see from formula (2.7) that the condition $\partial * \omega=0$ on $M$ is equivalent to

$$
I_{a}:=\sum_{a=1}^{n} \frac{\partial\left(G g^{\bar{a} b}\right)}{\partial z_{b}}=0, a=1, \ldots, n, \quad \text { on } M,
$$

so that the Laplacian $\Delta$ on functions $u$ has the form

$$
\Delta u=-2 \sum_{a, b=1}^{n} g^{\bar{a} b} \frac{\partial^{2} u}{\partial \bar{z}_{a} \partial z_{b}} .
$$

Thus 1. and 2. follow from formulas (2.1) and (2.3).

\section{Remark 3.2.}

1. Under the condition in Theorem 3.1 in case $\operatorname{dim} M=n=2$, if $\frac{\partial^{2} \lambda}{\partial t \partial \hat{t}}\left(t_{0}\right)=0$, then $\partial D\left(t_{0}\right)$ is Levi flat. If $n>2$, this conclusion is not necessarily true.

2. As mentioned in section $2, k_{2}(t, z)$ is a well-defined function on $\partial \mathcal{D}$ for any Hermitian metric $d s^{2}$ on $M$; i.e., it is independent of the local coordinates $z$. This is not the case for $K_{2}(t, z)$. From (2.1) and (3.3) it follows that $K_{2}(t, z)$ is a well-defined function on $\partial \mathcal{D}$ if and only if $d s^{2}$ satisfies condition (3.1).

We now turn to the question as to when $\frac{\partial^{2} \lambda}{\partial t \partial \bar{t}} \leq 0$; i.e., the subharmonicity of $-\lambda(t)$. We begin with the following:

Theorem 3.2. Assume that $d s^{2}$ satisfies condition (3.1). If $\mathcal{D}$ is pseudoconvex in $B \times M$, then $-\lambda(t)$ is subharmonic on $B$.

Proof. From the second variation formula for $\lambda(t)$ in 2. of Theorem 3.1 it suffices to prove that $K_{2}(t, z) \geq 0$ on $\partial \mathcal{D}$.

Fix $\left(t_{0}, z_{0}\right) \in \partial \mathcal{D}$; we may assume $\left(t_{0}, z_{0}\right)=(0,0)$. We choose coordinates $z=\left(z_{1}, \ldots, z_{n}\right)$ in a neighborhood $V$ of 0 so that $g_{\bar{a} b}(0)=\delta_{a b}$. Let $\psi(t, z)$ be a defining function for $\mathcal{D}$. We take a sufficiently small disk $B_{0} \subset B$ centered at $t=0$ so that $\psi(t, z)$ is defined in $B_{0} \times V$. Then from Theorem 3.1 we have

$$
\begin{gathered}
K_{2}(0,0)=\left\|\nabla_{z} \psi\right\|^{-3} L \psi(0,0) \\
:=\left\|\nabla_{z} \psi\right\|^{-3}\left[\frac{\partial^{2} \psi}{\partial t \partial \bar{t}}\left\|\nabla_{z} \psi\right\|^{2}-2 \Re\left\{\frac{\partial \psi}{\partial t} \sum_{a=1}^{n} \frac{\partial \psi}{\partial \bar{z}_{a}} \frac{\partial^{2} \psi}{\partial \bar{t} \partial z_{a}}\right\}+\left|\frac{\partial \psi}{\partial t}\right|^{2} \Delta_{z} \psi\right] .
\end{gathered}
$$


Here $\Delta_{z} \psi=\sum_{\alpha=1}^{n} \frac{\partial^{2} \psi}{\partial z_{\alpha} \partial \bar{z}_{\alpha}}, \quad \nabla_{z} \psi=\left(\frac{\partial \psi}{\partial z_{1}}, \cdots, \frac{\partial \psi}{\partial z_{n}}\right)$ and the terms on the right hand side are evaluated at $(t, z)=(0,0)$. Let $\mathcal{D}_{0}:=\mathcal{D} \cap\left(B_{0} \times V\right)$. Then $\psi(t, z)$ is a defining function for $\mathcal{D}_{0}$ near $\partial \mathcal{D} \cap \mathcal{D}_{0}$; i.e., $\psi$ is negative on $\mathcal{D}_{0}$ and $\nabla_{z} \psi(t, z) \neq 0$ on $\partial \mathcal{D} \cap \mathcal{D}_{0}$. For $j \in\{1, \ldots, n\}$, let

$$
\mathcal{D}_{0, j}:=\mathcal{D}_{0} \cap\left\{z_{1}=\ldots=\widehat{z_{j}}=\ldots=z_{n}=0\right\} .
$$

Since $\mathcal{D}_{0}$ is pseudoconvex in $\mathbb{C}^{n+1}$, it follows that $\mathcal{D}_{0, j}$ is pseudoconvex in $\mathbb{C}^{2}$ in the variables $\left(t, z_{j}\right)$; hence, for $j=1, \ldots, n$,

$$
\frac{\partial^{2} \psi}{\partial t \partial \bar{t}}\left|\frac{\partial \psi}{\partial z_{j}}\right|^{2}-2 \Re\left\{\frac{\partial \psi}{\partial t} \frac{\partial \psi}{\partial \bar{z}_{j}} \frac{\partial^{2} \psi}{\partial \bar{t} \partial z_{j}}\right\}+\left|\frac{\partial \psi}{\partial t}\right|^{2} \frac{\partial^{2} \psi}{\partial \bar{z}_{j} \partial z_{j}} \geq 0
$$

at the point $(0,0)$. Summing up from $j=1, \ldots, n$, we get

$$
L \psi(0,0) \geq 0 .
$$

Using (3.5) yields the result.

Remark 3.3. We consider the following condition on the metric $d s^{2}$ on $M$ :

$$
\mathcal{W}=\mathcal{W}_{d s^{2}}:=\frac{1}{i} \bar{\partial} \partial * \omega-\|\partial * \omega\|^{2} \frac{\omega^{n}}{n !} \geq 0
$$

as an $(n, n)$ form on $M$. This is a weaker condition than (3.1). We can prove from (2.3) that, if $d s^{2}$ satisfies condition $(W)$ and if $\mathcal{D}$ is pseudoconvex in $B \times M$, then $-\lambda(t)$ is subharmonic on $B$. We put $\mathcal{W}=W(z) \frac{\omega^{n}}{n !}$. In local coordinates, $W(z)$ has the form

$$
W(z)=\frac{1}{G} \sum_{\alpha, \beta=1}^{n}\left[\frac{\partial^{2}\left(G g^{\bar{\alpha} \beta}\right)}{\partial \bar{z}_{\alpha} \partial z_{\beta}}-\frac{g_{\alpha \bar{\beta}}}{G} \overline{\left(\sum_{l=1}^{n} \frac{\partial\left(G g^{\bar{\alpha} l}\right)}{\partial z_{l}}\right)}\left(\sum_{k=1}^{n} \frac{\partial\left(G g^{\bar{\beta} k}\right)}{\partial z_{k}}\right)\right] .
$$

Using standard notation from classical differential geometry, we define complex Christoffel symbols

$$
\Gamma_{\lambda \beta}^{\alpha}:=\sum_{\gamma=1}^{n} g^{\bar{\gamma} \alpha} \frac{\partial g_{\beta \bar{\gamma}}}{\partial z_{\lambda}}
$$

and complex torsion

$$
T_{\lambda \beta}^{\gamma}:=\Gamma_{\lambda \beta}^{\gamma}-\Gamma_{\beta \lambda}^{\gamma} .
$$

Summing, we define $T_{\alpha}:=\sum_{\lambda=1}^{n} T_{\lambda \alpha}^{\lambda}$. Then

$$
W(z)=\sum_{\alpha, \beta=1}^{n} g^{\bar{\beta} \alpha} \frac{\partial T_{\alpha}}{\partial \bar{z}_{\beta}}
$$


which is a type of scalar curvature function on $M$. Thus the condition $(W)$ means that this scalar curvature $W(z)$ is nonnegative. As an example, on the unit ball $M=\left\{z \in \mathbb{C}^{n}:\|z\|<1\right\}$ in $\mathbb{C}^{n}, n \geq 2$, take $d s^{2}:=\frac{|d z|^{2}}{\left(1-\|z\|^{2}\right)^{2}}$, where $|d z|^{2}$ is the Euclidean metric in $\mathbb{C}^{n}$. Then $W(z)=2(n-1)\left(n-(n-1)\|z\|^{2}\right) \geq$ $2(n-1)>0$ on $M$. On the other hand, the special Hopf manifold $\mathbb{H}_{n}, n \geq 2$ (see definition (6.38) in section 6) admits the Hermitian metric $d s^{2}:=\frac{|d z|^{2}}{\|z\|^{2}}$. A calculation shows that $W(z)=-(n-1)^{2}<0$ on $\mathbb{H}_{n}$. We plan to investigate condition $(W)$ in a future paper.

J-C. Joo [6] has a generalization of our second variation formula (2.3) to certain almost complex manifolds.

\section{Rigidity.}

We continue under the same hypotheses: $M$ is an $n$-dimensional complex manifold equipped with a Hermitian metric $d s^{2} ; \mathcal{D}=\cup_{t \in B}(t, D(t)) \subset B \times M$ is a $C^{\infty}$ variation of domains $D(t)$ in $M$ each containing a fixed point $p_{0}$ and with $\partial D(t)$ of class $C^{\infty}$ for $t \in B$; and $c$ is a nonnegative $C^{\infty}$ function on $M$.

Throughout this section we will assume that

(1) $d s^{2}$ satisfies condition (3.1) on $M$;

(2) $\mathcal{D}$ is pseudoconvex in $B \times M$.

Lemma 4.1 (Rigidity). If there exists $t_{0} \in B$ at which $\frac{\partial^{2} \lambda}{\partial t \partial \bar{t}}\left(t_{0}\right)=0$, then $\frac{\partial g}{\partial t}\left(t_{0}, z\right) \equiv 0$ for $z \in D\left(t_{0}\right)$ provided at least one of the following conditions hold:

i) $c(z) \not \equiv 0$ on $D\left(t_{0}\right)$;

ii) $\partial D\left(t_{0}\right)$ is not Levi flat.

Proof. Since $\mathcal{D}$ is pseudoconvex in $B \times M$, Theorems 3.1 and 3.2 imply that $k_{2}(t, z) \geq 0$ on $\partial \mathcal{D}$ and we obtain the following estimate from (3.2):

$$
\frac{\partial^{2} \lambda}{\partial t \partial \bar{t}}\left(t_{0}\right) \leq \frac{-c_{n}}{2^{n-1}}\left\{2\left\|\bar{\partial} \frac{\partial g}{\partial t}\right\|_{D\left(t_{0}\right)}^{2}+\left\|\sqrt{c} \frac{\partial g}{\partial t}\right\|_{D\left(t_{0}\right)}^{2}\right\} .
$$

Thus if the left hand side $\frac{\partial^{2} \lambda}{\partial t \partial \bar{t}}\left(t_{0}\right)=0$, then (see (2.3) $) k_{2}\left(t_{0}, z\right) \equiv 0$ on $\partial D\left(t_{0}\right)$, and each term on the right in (4.1) must vanish. Hence

1. $\bar{\partial} \frac{\partial g}{\partial t}\left(t_{0}, z\right) \equiv 0$ on $D\left(t_{0}\right)$; 
2. $\sqrt{c} \frac{\partial g}{\partial t}\left(t_{0}, z\right) \equiv 0$ on $D\left(t_{0}\right)$.

In particular, 1 . says that $\frac{\partial g}{\partial t}\left(t_{0}, z\right)$ is holomorphic on $D\left(t_{0}\right)$. First assume i) holds. Since $c$ is of class $C^{\infty}, c(z) \not \equiv 0$ on $D\left(t_{0}\right)$ together with 2. implies that the holomorphic function $\frac{\partial g}{\partial t}\left(t_{0}, z\right)$ vanishes on an open set in $D\left(t_{0}\right)$ and hence vanishes identically on $D\left(t_{0}\right)$.

Now assume ii) holds but i) does not; i.e., $c(z) \equiv 0$ on $D\left(t_{0}\right)$. We want to show that $\frac{\partial g}{\partial t}\left(t_{0}, \cdot\right) \equiv 0$ on $\partial D\left(t_{0}\right)$. To see this, fix a point $q_{0} \in \partial D\left(t_{0}\right)$. Using local coordinates $z=\left(z_{1}, \ldots, z_{n}\right)$ in a neighborhood $V$ of $q_{0}$ where $z=0$ corresponds to $q_{0}$, we can assume that $g_{a \bar{b}}(0)=\delta_{a b}$. We show that $\frac{\partial g}{\partial t}\left(t_{0}, 0\right)=0$.

Since $\frac{\partial g}{\partial t}\left(t_{0}, z\right)$ is holomorphic in $D\left(t_{0}\right)$ and of class $C^{\infty}$ on $\overline{D\left(t_{0}\right)}$, we have $\bar{\partial} \frac{\partial g}{\partial t}=0$ on $\partial D\left(t_{0}\right)$. Then formula (2.6)

$$
\begin{aligned}
& k_{2}\left(t_{0}, z\right) \cdot\left(\sum_{a, b=1}^{n} g^{\bar{a} b} \frac{\partial g}{\partial \bar{z}_{a}} \frac{\partial g}{\partial z_{b}}\right)^{3 / 2}=-\frac{\partial^{2} g}{\partial t \partial \bar{t}}\left(\sum_{a, b=1}^{n} g^{\bar{a} b} \frac{\partial g}{\partial \bar{z}_{a}} \frac{\partial g}{\partial z_{b}}\right) \\
& +2 \Re\left[\left(\sum_{a, b=1}^{n} g^{\bar{a} b} \frac{\partial^{2} g}{\partial z_{b} \partial \bar{t}} \frac{\partial g}{\partial \bar{z}_{a}}\right) \frac{\partial g}{\partial t}\right]-\left|\frac{\partial g}{\partial t}\right|^{2}\left(\sum_{a, b=1}^{n} g^{\bar{a} b} \frac{\partial^{2} g}{\partial \bar{z}_{a} \partial z_{b}}\right)
\end{aligned}
$$

simplifies at the point $\left(t_{0}, 0\right)$ to

$$
k_{2}\left(t_{0}, 0\right) \cdot\|\nabla g\|^{3}+\frac{\partial^{2} g}{\partial t \partial \bar{t}}\|\nabla g\|^{2}+\left|\frac{\partial g}{\partial t}\right|^{2} \sum_{i=1}^{n} \frac{\partial^{2} g}{\partial z_{i} \partial \bar{z}_{i}}=0 .
$$

Since $k_{2}\left(t_{0}, z\right)=0$ for $z \in \partial D\left(t_{0}\right)$, we have

$$
\frac{\partial^{2} g}{\partial t \partial \bar{t}}\|\nabla g\|^{2}+\left|\frac{\partial g}{\partial t}\right|^{2} \sum_{i=1}^{n} \frac{\partial^{2} g}{\partial z_{i} \partial \bar{z}_{i}}=0 \quad \text { at }\left(t_{0}, 0\right) .
$$

Note that $-g$ is a defining function for $\mathcal{D}$. Using pseudoconvexity of $\mathcal{D}$ at $\left(t_{0}, z\right), z \in \partial D\left(t_{0}\right)$, and using $\bar{\partial} \frac{\partial g}{\partial t}=0$ on $\partial D\left(t_{0}\right)$, it follows that

$$
\frac{\partial^{2} g}{\partial t \partial \bar{t}}\left|\frac{\partial g}{\partial z_{i}}\right|^{2}+\left|\frac{\partial g}{\partial t}\right|^{2} \frac{\partial^{2} g}{\partial z_{i} \partial \bar{z}_{i}} \leq 0 \quad \text { on } \quad \partial D\left(t_{0}\right) \cap V
$$

for each $i=1, \ldots, n$. Now since $\partial * \omega \equiv 0$ on $M$, it follows from (3.4) and $g_{a \bar{b}}(0)=\delta_{a b}$ that the Laplacian $\Delta$ on functions $u$ at $z=0$ has the form

$$
\Delta u(0)=-2 \sum_{i=1}^{n} \frac{\partial^{2} u}{\partial z_{i} \partial \bar{z}_{i}}(0)
$$


We are assuming that $c \equiv 0$ on $D\left(t_{0}\right)$ and hence on $\partial D\left(t_{0}\right)$; thus $\Delta g\left(t_{0}, z\right)=0$ for $z \in D\left(t_{0}\right)$ except at the pole $p_{0}$. We thus have

$$
\sum_{i=1}^{n} \frac{\partial^{2} g}{\partial z_{i} \partial \bar{z}_{i}}\left(t_{0}, 0\right)=0 .
$$

Using (4.2), we conclude that $\frac{\partial^{2} g}{\partial t \partial \bar{t}}\|\nabla g\|^{2}=0$ at $\left(t_{0}, 0\right)$. Hence

$$
\left|\frac{\partial g}{\partial t}\right|^{2} \frac{\partial^{2} g}{\partial z_{i} \partial \bar{z}_{i}} \leq 0 \quad \text { at }\left(t_{0}, 0\right), \quad i=1, \ldots, n
$$

From the assumption ii), $\frac{\partial^{2} g}{\partial z_{i} \partial \bar{z}_{i}}\left(t_{0}, 0\right)>0$ for some $i$, and we conclude that $\frac{\partial g}{\partial t}=0$ at $\left(t_{0}, 0\right)$. Thus the holomorphic function $\frac{\partial g}{\partial t}$ vanishes identically on $\partial D\left(t_{0}\right)$, and hence on $D\left(t_{0}\right)$.

Corollary 4.1 (Trivial variation). If $\lambda(t)$ is harmonic in $B$ and i) or ii) in Lemma 4.1 holds, then $\mathcal{D}=B \times D(0)$.

Proof. By Lemma 4.1, $\frac{\partial g}{\partial t}=0$ on $\mathcal{D}$, i.e., $g(t, z)$ does not depend on $t \in B$. By analytic continuation it suffices to prove that there exists a small disk $B_{0} \subset B$ centered at $t=0$ such that $\left.\mathcal{D}\right|_{B_{0}}=B_{0} \times D(0)$, where $\left.\mathcal{D}\right|_{B_{0}}=$ $\cup_{t \in B_{0}}(t, D(t))$. If not, there exists a sequence $\left\{t_{n}\right\}$ such that $t_{n} \neq 0, t_{n} \rightarrow 0$ as $n \rightarrow \infty$, and $D\left(t_{n}\right) \neq D(0)$. Thus we can find a point $z_{n} \in(\partial D(0) \cap$ $\left.D\left(t_{n}\right)\right) \cup\left(D(0) \cap \partial D\left(t_{n}\right)\right)$ for each $n$. If $z_{n} \in \partial D(0) \cap D\left(t_{n}\right)$, then, $g\left(0, z_{n}\right)=0$ and $g\left(t_{n}, z_{n}\right)>0$, which is a contradiction; if $z_{0} \in D(0) \cap \partial D\left(t_{n}\right), g\left(0, z_{n}\right)>0$ and $g\left(t_{n}, z_{n}\right)=0$ which is again a contradiction.

We next consider the following set-up. Let $F: B \times M \rightarrow M$ be a holomorphically varying, one-parameter family of automorphisms of $M$; i.e., $F(t, z)$ is holomorphic in $(t, z)$ with $\frac{\partial F}{\partial t} \neq 0$ for $(t, z) \in B \times M$, and, for each $t \in B$, the mapping $F_{t}: M \rightarrow M$ via $F_{t}(z):=F(t, z)$ is an automorphism of $M$. Then the mapping $T: B \times M \rightarrow B \times M$ defined as

$$
T(t, z)=(t, w):=(t, F(t, z))
$$

provides a fiber-wise automorphism of $M$; i.e., for each $t \in B$, the map $w=F(t, z)$ is an automorphism of $M$.

Let $d s^{2}$ be a Hermitian metric on $M$ satisfying condition (3.1) and let $c(z) \geq 0$ be a $C^{\infty}$ function on $M$. Fix a pseudoconvex domain $D \Subset M$ and let $\mathcal{D}:=T(B \times D)$. This is a variation of pseudoconvex domains $D(t)=F(t, D)$ in the image space $B \times M$ of $T$. Assume there exists a common point $w_{0}$ in each domain $D(t), t \in B$. Let $g(t, w)$ and $\lambda(t)$ denote the $c$-Green function and $c$-Robin constant of $\left(D(t), w_{0}\right)$ for the Laplacian $\Delta$ associated to $d s^{2}$. We obtain the following fundamental result, utilizing the rigidity lemma, Lemma 4.1 . 


\section{Corollary 4.2. If}

$$
\frac{\partial^{2} \lambda}{\partial t \partial \bar{t}}\left(t_{0}\right)=0
$$

at some $t_{0} \in B$, and either $\left\{z \in D\left(t_{0}\right): c(z) \neq 0\right\} \neq \emptyset$ or $\partial D\left(t_{0}\right)$ (or equivalently $\partial D$ ) is not Levi flat, then there exists a nonvanishing holomorphic vector field $\Theta$ on $M$ which is tangential on $\partial D ; i . e$. , the entire integral curve $I\left(z_{0}\right)$ associated to $\Theta$ for any initial point $z_{0} \in \partial D$ lies on $\partial D$.

Proof. We may take $t_{0}=0$. For fixed $t$, we let $\phi(t, w):=F_{t}^{-1}(w)$ so that $z=\phi(t, w)$ if $w=F(t, z)$. Let $z_{0}(t):=\phi\left(t, w_{0}\right)$ for $t \in B$; thus, in the $(t, z)$-coordinates in $B \times M$ we have a constant variation of domains $t \rightarrow D(t) \equiv D$ with a varying family of poles $z_{0}(t)$; letting $c(t, z):=c(F(t, z))$ and $d s_{t}^{2}(z):=F(t, \cdot)^{*}\left(d s^{2}(w)\right)$ (the pull-back of $d s^{2}$ by $\left.F(t, \cdot)\right)$ - note this is a varying family of Hermitian metrics on the fixed domain $D$ - we have the $c(t, z)$-Green function $\mathcal{G}(t, z)$ for $\left(D, z_{0}(t)\right)$ for the Laplacian $\tilde{\Delta}_{t}$ associated to $d s_{t}^{2}(z)$. Indeed,

$$
\mathcal{G}(t, z):=g(t, F(t, z))
$$

satisfies $\Delta g(t, w)=\tilde{\Delta}_{t} g(t, F(t, z))$ by the invariance of the Hodge-* operator under holomorphic mappings.

The assumption that $\frac{\partial^{2} \lambda}{\partial t \partial \bar{t}}(0)=0$ implies $\frac{\partial g}{\partial t}(0, w) \equiv 0$ on $D(0)$ by the rigidity lemma. For $p_{0} \in M$, we let $q_{0}=F\left(0, p_{0}\right)$ and we choose local coordinates $z$ on a coordinate neighborhood $U$ of $p_{0}$ and $w$ on a coordinate neighborhood $V$ of $q_{0}$ and write

$$
z=\phi(t, w)=\left(z_{1}, \ldots, z_{n}\right)=\left(\phi_{1}(t, w), \ldots, \phi_{n}(t, w)\right) .
$$

Using these coordinates and the relation $\mathcal{G}(t, \phi(t, w))=g(t, w)$, and noting that each $\phi_{j}, j=1, \ldots, n$ is holomorphic, the condition $\frac{\partial g}{\partial t}(0, w) \equiv 0$ on $D(0)$ becomes

$$
\frac{\partial \mathcal{G}}{\partial t}(0, z)+\sum_{k=1}^{n} \frac{\partial \mathcal{G}}{\partial z_{k}}(0, z) \frac{\partial \phi_{k}}{\partial t}(0, F(0, z))=0
$$

for $z \in D$. Since $g(t, w)$ is of class $C^{\infty}$ on $\overline{\mathcal{D}}, \mathcal{G}(t, \phi(t, w))$ is of class $C^{\infty}$ on $B \times \bar{D}$. Thus, the above equality is valid for $z \in \bar{D}$. Since $\mathcal{G}(t, z)=0$ for $z \in \partial D$ for all $t \in B$, we have $\frac{\partial \mathcal{G}}{\partial t}(0, z)=0$ for $z \in \partial D$, and hence

$$
\sum_{k=1}^{n} \frac{\partial \mathcal{G}}{\partial z_{k}}(0, z) \frac{\partial \phi_{k}}{\partial t}(0, F(0, z))=0, \quad \text { for } z \in \partial D .
$$

The vector field defined by

$$
\Theta(z):=\sum_{k=1}^{n} \frac{\partial \phi_{k}}{\partial t}(0, F(0, z)) \frac{\partial}{\partial z_{k}}
$$


in each coordinate neighborhood $U$ with coordinates $z$ (and coordinate neighborhood $V$ for $w=\phi(t, z), z \in U$ ) is easily seen to be a holomorphic vector field on all of $M$. Furthermore, since $\frac{\partial F}{\partial t} \neq 0$ on $B \times M$ and $\phi^{-1}=F$, we have $\Theta(z) \neq 0$ on $M$.

We shall verify the property that the entire integral curve $I\left(z_{0}\right)$ associated to $\Theta$ for any initial point $z_{0} \in \partial D$ lies on $\partial D$, i.e., writing $\Theta(z)=$ $\sum_{k=1}^{n} \zeta_{k}(z) \frac{\partial}{\partial z_{k}}$, if we consider the ordinary differential equation

$$
\frac{d Z_{j}}{d t}=\zeta_{j}\left(Z_{1}, \ldots, Z_{n}\right), \quad j=1, \ldots, n,
$$

on $M$, then the solution curve $Z(t)=\left(Z_{1}(t), \ldots, Z_{n}(t)\right), t \in \mathbb{C}_{t}$ with initial value $Z(0)=z_{0}$ always lies on $\partial D$.

To see this, we fix such a $z_{0}$ which we take to be 0 . Since the $c(0, z)$-Green function $\mathcal{G}(0, z)$ vanishes on $\partial D$, the vector

$$
\left(\frac{\partial \mathcal{G}}{\partial z_{1}}(0, z), \ldots, \frac{\partial \mathcal{G}}{\partial z_{n}}(0, z)\right)
$$

is a nonvanishing complex normal vector to $\partial D$ at $z$. By (4.3) we have $\sum_{k=1}^{n} \zeta_{k}(z) \frac{\partial \mathcal{G}(0, z)}{\partial z_{k}}=0$ on $\partial D$. Thus, at each point $z \in \partial D$, the vector $\left(\zeta_{1}(z), \ldots, \zeta_{n}(z)\right)$ is a holomorphic tangent vector. This observation gives the intuitive reason for the property of the solution $Z(t)$ of the system of equations (4.5) in the statement of the corollary.

To verify this property of $Z(t)$, we set up local coordinates $z=\left(z_{1}, \ldots, z_{n}\right)$ near the point $z_{0}$ where $z_{k}=x_{k}+i y_{k}$ so that

$$
z=(x, y)=\left(x_{1}, y_{1}, \ldots, x_{n}, y_{n}\right) \in \mathbb{R}^{2 n} .
$$

We let $z_{0}$ correspond to $z=0$ and define $\Psi(z)=\Psi(x, y):=\mathcal{G}(0, z)$. Then $\Psi$ is a $C^{\infty}$ real-valued function near $\partial D$ with $\Psi=0$ and $\frac{\partial \Psi}{\partial n_{z}}<0$ on $\partial D$, where $n_{z}$ is the unit outer normal vector to $\partial D$ at $(x, y)$; i.e.,

$$
\operatorname{Grad} \Psi_{(x, y)}=\left(\frac{\partial \Psi}{\partial x_{1}}, \frac{\partial \Psi}{\partial y_{1}}, \ldots, \frac{\partial \Psi}{\partial x_{n}}, \frac{\partial \Psi}{\partial y_{n}}\right)
$$

is a normal vector to $\partial D$ at $(x, y)$.

The holomorphic vector field

$$
\Theta:=\sum_{k=1}^{n} \zeta_{k}(z) \frac{\partial}{\partial z_{k}} \quad \text { on } M
$$

satisfies

$$
\sum_{k=1}^{n} \zeta_{k}(z) \frac{\partial \Psi}{\partial z_{k}}(z)=0 \quad \text { on } \partial D
$$


We set

$$
\zeta_{k}(z):=a_{k}(x, y)+i b_{k}(x, y), \quad k=1, \ldots, n,
$$

where $a_{k}, b_{k}$ are real-valued on $M$. Since $\Psi$ is real-valued, it follows that

$$
\sum_{k=1}^{n}\left(a_{k} \frac{\partial \Psi}{\partial x_{k}}+b_{k} \frac{\partial \Psi}{\partial y_{k}}\right)+i \sum_{k=1}^{n}\left(b_{k} \frac{\partial \Psi}{\partial x_{k}}-a_{k} \frac{\partial \Psi}{\partial y_{k}}\right)=0 \quad \text { on } \partial D .
$$

Consequently, if we set

$$
\begin{aligned}
& \Theta_{1}:=\Re \Theta=\sum_{k=1}^{n}\left(a_{k} \frac{\partial}{\partial x_{k}}+b_{k} \frac{\partial}{\partial y_{k}}\right) \\
& \Theta_{2}:=\Im \Theta=\sum_{k=1}^{n}\left(b_{k} \frac{\partial}{\partial x_{k}}-a_{k} \frac{\partial}{\partial y_{k}}\right),
\end{aligned}
$$

then $\Theta_{1}, \Theta_{2}$ are real vector fields on $M$ which are tangential on $\partial D$.

We consider the integral curve $Z(t)=\left(Z_{1}(t), \ldots, Z_{n}(t)\right), t \in \mathbb{C}$, with initial value $Z(0)=z_{0}$ as mentioned above, so that

$$
\frac{d Z_{k}(t)}{d t}=\zeta_{k}(Z(t)), \quad t \in \mathbb{C}, \quad k=1, \ldots, n .
$$

We write $t=t_{1}+i t_{2}$ where $\left(t_{1}, t_{2}\right) \in \mathbb{R}^{2} ; Z_{k}(t)=x_{k}\left(t_{1}, t_{2}\right)+i y_{k}\left(t_{1}, t_{2}\right)$ and

$$
x\left(t_{1}, t_{2}\right)=\left(x_{k}\left(t_{1}, t_{2}\right)\right)_{k=1, \ldots, n}, \quad y\left(t_{1}, t_{2}\right)=\left(y_{k}\left(t_{1}, t_{2}\right)\right)_{k=1, \ldots, n}
$$

for $\left(t_{1}, t_{2}\right) \in \mathbb{R}^{2}$, so that $Z(t)=x\left(t_{1}, t_{2}\right)+i y\left(t_{1}, t_{2}\right)$. Since $Z_{k}(t)$ is holomorphic, we have $\frac{d Z_{k}(t)}{d t}=\frac{\partial Z_{k}(t)}{\partial t_{1}}$ and hence

$$
\text { (1) }\left\{\begin{array}{l}
\frac{\partial x_{k}\left(t_{1}, t_{2}\right)}{\partial t_{1}}=a_{k}\left(x\left(t_{1}, t_{2}\right), y\left(t_{1}, t_{2}\right)\right) \\
\frac{\partial y_{k}\left(t_{1}, t_{2}\right)}{\partial t_{1}}=b_{k}\left(x\left(t_{1}, t_{2}\right), y\left(t_{1}, t_{2}\right)\right)
\end{array}\right.
$$

for $\left(t_{1}, t_{2}\right) \in \mathbb{R}^{2}$. Analogously, $\frac{d Z_{k}(t)}{d t}=\frac{1}{i} \frac{\partial Z_{k}(t)}{\partial t_{2}}$ implies

$$
\left\{\begin{array}{l}
\frac{\partial x_{k}\left(t_{1}, t_{2}\right)}{\partial t_{2}}=-b_{k}\left(x\left(t_{1}, t_{2}\right), y\left(t_{1}, t_{2}\right)\right) \\
\frac{\partial y_{k}\left(t_{1}, t_{2}\right)}{\partial t_{2}}=a_{k}\left(x\left(t_{1}, t_{2}\right), y\left(t_{1}, t_{2}\right)\right)
\end{array}\right.
$$

for $\left(t_{1}, t_{2}\right) \in \mathbb{R}^{2}$. If we set $t_{2}=0$ in $(1)$, then $\left(x\left(t_{1}, 0\right), y\left(t_{1}, 0\right)\right)$ satisfies

$$
\left\{\begin{aligned}
\frac{d x_{k}\left(t_{1}, 0\right)}{d t_{1}} & =a_{k}\left(x\left(t_{1}, 0\right), y\left(t_{1}, 0\right)\right) \\
\frac{d y_{k}\left(t_{1}, 0\right)}{d t_{1}} & =b_{k}\left(x\left(t_{1}, 0\right), y\left(t_{1}, 0\right)\right)
\end{aligned}\right.
$$


for $t_{1} \in(-\infty, \infty)$ and $(x(0,0), y(0,0))=z_{0}$. Thus $\left(x\left(t_{1}, 0\right), y\left(t_{1}, 0\right)\right)$ is an integral curve for $\Theta_{1}$ with initial vaue $z_{0}$. Since $\Theta_{1}$ is tangential on $\partial D$, it follows that

$$
\left(x\left(t_{1}, 0\right), y\left(t_{1}, 0\right)\right) \in \partial D, \quad t_{1} \in(-\infty, \infty) .
$$

Next, considering equation (2) with fixed $t_{1} \in(-\infty,+\infty)$ and variable $t_{2} \in$ $(-\infty, \infty)$, we see that $\left(x\left(t_{1}, t_{2}\right), y\left(t_{1}, t_{2}\right)\right)$ is an integral curve for $\Theta_{2}$ with initial vaue $\left(x\left(t_{1}, 0\right), y\left(t_{1}, 0\right)\right) \in \partial D$. Since $\Theta_{2}$ is tangential on $\partial D$, we have

$$
\left(x\left(t_{1}, t_{2}\right), y\left(t_{1}, t_{2}\right)\right) \in \partial D, \quad t_{2} \in(-\infty, \infty) .
$$

Hence

$$
Z(t)=x\left(t_{1}, t_{2}\right)+i y\left(t_{1}, t_{2}\right) \in \partial D, \quad t \in \mathbb{C},
$$

as required.

We remark that the hypothesis of the corollary is satisfied if, for example, $c>0$ in $M$.

\section{Remark 4.1.}

1. By an elementary topological argument, the corollary immediately implies that the integral curve $I\left(z_{0}\right)$ of the vector field $\Theta$ with initial value $z_{0} \in D\left(\bar{D}^{c}\right)$ always lies in $D\left(\bar{D}^{c}\right)$. However, this does not necessarily imply that $I\left(z_{0}\right) \Subset D\left(\bar{D}^{c}\right)$.

2. If $\partial D$ contains at least one strictly pseudoconvex point, then $\frac{\partial^{2} \lambda}{\partial t \partial \bar{t}}(t)>0$ on $B$.

3. In the case where $\operatorname{dim} M=n=2, \frac{\partial^{2} \lambda}{\partial t \partial \bar{t}}\left(t_{0}\right)=0$ for some $t_{0} \in B$ implies that $\partial D$ is Levi flat.

Corollary 4.2 is fundamental for the applications in this paper. We now prove a partial converse. First we remark that for any fixed $t \in B$,

$$
\Theta(t, z):=\sum_{k=1}^{n} \frac{\partial \phi_{k}}{\partial t}(t, F(t, z)) \frac{\partial}{\partial z_{k}}
$$

is a holomorphic vector field on all of $M$. Thus

$$
\Theta: t \in B \rightarrow \Theta(t, z)
$$

is a variation of holomorphic vector fields on $M$. We have the following result. 
Corollary 4.3. Assume that either $\{z \in M: c(z) \neq 0\}$ is a dense, open set in $M$ or that $\partial D \subset M$ is not Levi flat. Then $\frac{\partial^{2} \lambda}{\partial t \partial \bar{t}}\left(t_{0}\right)=0$ for some $t_{0} \in B$ if and only if $\Theta\left(t_{0}, z\right)$ is a tangential vector field on $\partial D$.

Proof. We verified the sufficiency in the proof of the previous corollary. We verify the necessity. From the relation $\mathcal{G}(t, \phi(t, w))=g(t, w)$, for any fixed $t \in B$,

$$
\frac{\partial g}{\partial t}(t, w)=\frac{\partial \mathcal{G}}{\partial t}(t, z)+\sum_{k=1}^{n} \frac{\partial \mathcal{G}}{\partial z_{k}}(t, z) \frac{\partial \phi_{k}}{\partial t}(t, F(t, z))
$$

for $z \in \bar{D}$ and $w=F(t, z)$. Since $\mathcal{G}(t, z)=0$ on $\partial D$ for all $t \in B$, it follows that

$$
\frac{\partial g}{\partial t}(t, w)=\sum_{k=1}^{n} \frac{\partial \mathcal{G}}{\partial z_{k}}(t, z) \frac{\partial \phi_{k}}{\partial t}(t, F(t, z)), \quad \text { for } z \in \partial D .
$$

Suppose that for some $t_{0} \in B, \Theta\left(t_{0}, z\right)$ is tangential on $\partial D$. Since $-\mathcal{G}\left(t_{0}, z\right)$ is a defining function for $\partial D$, this is equivalent to

$$
\sum_{k=1}^{n} \frac{\partial \mathcal{G}}{\partial z_{k}}\left(t_{0}, z\right) \frac{\partial \phi_{k}}{\partial t}\left(t_{0}, F\left(t_{0}, z\right)\right)=0, \quad \text { for } z \in \partial D,
$$

and hence

$$
\frac{\partial g}{\partial t}\left(t_{0}, w\right)=0, \quad \text { for } w \in \partial D\left(t_{0}\right) .
$$

Since $\frac{\partial g}{\partial t}\left(t_{0}, w\right)$ is a continuous function on $\overline{D\left(t_{0}\right)}$ and is $c$-harmonic on all of $D\left(t_{0}\right)$, it follows from the uniqueness theorem that $\frac{\partial g}{\partial t}\left(t_{0}, w\right) \equiv 0$ on $D\left(t_{0}\right)$. From our second variation formula (3.2) we have

$$
\begin{aligned}
\frac{\partial^{2} \lambda}{\partial t \partial \bar{t}}\left(t_{0}\right) & =-c_{n} \int_{\partial D\left(t_{0}\right)} K_{2}\left(t_{0}, w\right) \sum_{a, b=1}^{n}\left(g^{\bar{a} b} \frac{\partial g}{\partial \bar{w}_{a}} \frac{\partial g}{\partial w_{b}}\right) d \sigma_{w} \\
-\frac{c_{n}}{2^{n-2}} & \left\{\left\|\bar{\partial} \frac{\partial g}{\partial t}\right\|_{D\left(t_{0}\right)}^{2}+\frac{1}{2}\left\|\sqrt{c} \frac{\partial g}{\partial t}\right\|_{D\left(t_{0}\right)}^{2}\right\} \\
& =-c_{n} \int_{\partial D\left(t_{0}\right)} K_{2}\left(t_{0}, w\right) \sum_{a, b=1}^{n}\left(g^{\bar{a} b} \frac{\partial g}{\partial \bar{w}_{a}} \frac{\partial g}{\partial w_{b}}\right) d \sigma_{w} .
\end{aligned}
$$

Thus to finish the proof it suffices to show that $K_{2}\left(t_{0}, w\right)=0$ on $\partial D\left(t_{0}\right)$.

Recall that $\mathcal{D}=T(B \times D)$ where $T(t, z)=(t, w)=(t, F(t, z))$ and we write $z=\phi(t, w)$. Let $\psi(z)$ be a defining function for $\partial D$ and set

$$
\begin{array}{ll}
\varphi(t, w):=\psi(\phi(t, w)) & \text { on } \mathcal{D} \text {; i.e., } \\
\psi(z)=\varphi(t, F(t, z)) & \text { on } B \times D .
\end{array}
$$


Then $\varphi(t, w)$ is a defining function for $\partial \mathcal{D}$. From the definition of $K_{2}(t, w)$, we can choose local coordinates $w$ so that

$$
K_{2}\left(t_{0}, w\right)\left\|\nabla_{w} \varphi\right\|^{3}=\frac{\partial^{2} \varphi}{\partial t \partial \bar{t}}\left\|\nabla_{w} \varphi\right\|^{2}-2 \Re\left\{\frac{\partial \varphi}{\partial t} \sum_{a=1}^{n} \frac{\partial \varphi}{\partial \bar{w}_{a}} \frac{\partial^{2} \varphi}{\partial \bar{t} \partial w_{a}}\right\}+\left|\frac{\partial \varphi}{\partial t}\right|^{2} \Delta_{w} \varphi .
$$

Writing

$$
\phi(t, w)=\left(\phi_{1}(t, w), \ldots, \phi_{n}(t, w)\right), \quad \text { for }(t, w) \in \mathcal{D}
$$

we have

$$
\frac{\partial \varphi}{\partial t}(t, w)=\sum_{i=1}^{n} \frac{\partial \psi}{\partial z_{i}}(t, \phi(t, w)) \frac{\partial \phi_{i}}{\partial t}(t, w) \quad \text { near } \partial \mathcal{D} .
$$

Since $\phi(t, w)$ is holomorphic in $(t, w)$, it follows that

$$
\frac{\partial^{2} \varphi}{\partial \bar{t} \partial t}=\sum_{i, j=1}^{n} \frac{\partial^{2} \psi}{\partial z_{i} \partial \overline{z_{j}}}\left(t, \phi(t, w) \overline{\left(\frac{\partial \phi_{j}}{\partial t}\right)} \frac{\partial \phi_{i}}{\partial t} \quad \text { near } \partial \mathcal{D} .\right.
$$

By assumption, $\Theta\left(t_{0}, z\right)$ is tangential on $\partial D$ so that

$$
\sum_{i=1}^{n} \frac{\partial \phi_{i}}{\partial t}\left(t_{0}, F\left(t_{0}, z\right)\right) \frac{\partial \psi}{\partial z_{i}}(z)=0 \quad \text { on } \partial D
$$

This implies

$$
\frac{\partial \varphi}{\partial t}\left(t_{0}, F\left(t_{0}, z\right)\right)=0 \quad \text { on } \partial D ;
$$

i.e., $\frac{\partial \varphi}{\partial t}\left(t_{0}, w\right)=0$ on $\partial D\left(t_{0}\right)$. Hence

$$
K_{2}\left(t_{0}, w\right)\left\|\nabla_{w} \varphi\right\|=\frac{\partial^{2} \varphi}{\partial t \partial \bar{t}}\left(t_{0}, w\right) \quad \text { on } \quad \partial D\left(t_{0}\right) .
$$

Again using (4.7) and the fact that $\psi=0$ on $\partial D$, we have

$$
\sum_{i=1}^{n} \frac{\partial \phi_{i}}{\partial t}\left(t_{0}, F\left(t_{0}, z\right)\right) \frac{\partial \psi}{\partial z_{i}}(z)=f(z) \psi(z) \text { near } \partial D
$$

where $f(z)$ is a $C^{\infty}$ function near $\partial D$. Since $F\left(t_{0}, z\right)$ is holomorphic in $z$ and $\psi=0$ on $\partial D$, if we differentiate both sides of the previous equation with respect to $\bar{z}_{j}$ we obtain

$$
\sum_{i=1}^{n} \frac{\partial \phi_{i}}{\partial t}\left(t_{0}, F\left(t_{0}, z\right)\right) \frac{\partial^{2} \psi}{\partial z_{i} \partial \bar{z}_{j}}(z)=f(z) \frac{\partial \psi}{\partial \bar{z}_{j}}(z) \text { on } \partial D .
$$


Multiplying by $\overline{\left(\frac{\partial \phi_{j}}{\partial t}\right)\left(t_{0}, F\left(t_{0}, z\right)\right)}$ and adding for $j=1, \ldots, n$ gives

$$
\sum_{i, j=1}^{n} \frac{\partial \phi_{i}}{\partial t} \overline{\left(\frac{\partial \phi_{j}}{\partial t}\right)} \frac{\partial^{2} \psi}{\partial z_{i} \partial \bar{z}_{j}}=f \sum_{j=1}^{n} \frac{\partial \psi}{\partial \bar{z}_{j}}(z) \overline{\left(\frac{\partial \phi_{j}}{\partial t}\right)} \quad \text { on } \partial D \text {. }
$$

Since $\psi$ is real-valued, formula (4.7) implies that the right-hand side of this equation vanishes on $\partial D$; thus (4.8) implies that $K_{2}\left(t_{0}, w\right)=0$ on $\partial D\left(t_{0}\right)$.

\section{Complex Lie Groups.}

We apply Corollary 4.2 of the previous section to the study of complex Lie groups. Let $M$ be a complex Lie group of complex dimension $n$ with identity e. We always take $M$ to be connected. By a theorem of Kazama, Kim and $\mathrm{Oh}$ [8], there always exists a Kähler metric on $M$; thus using Remark 3.1. we conclude that $M$ is equipped with a Hermitian metric $d s^{2}$ satisfying condition (3.1). We fix such a Hermitian metric and a strictly positive $C^{\infty}$ function $c=c(z)$ on $M$ throughout this section.

We recall some general properties of finite-dimensional complex Lie groups. Let $M$ be a complex Lie group of complex dimension $n$. We let $\mathfrak{X}$ denote the set of all left-invariant holomorphic vector fields $X$ on $M$ and we write $\exp t X$ for the integral curve of $X$ with initial value $e$. We note that for any $\zeta \in M$ the integral curve of $X$ with initial value $\zeta$ is given by $\zeta \exp t X$. Moreover, for $s, t \in \mathbb{C}$, we have the relations

$$
(\exp t X)^{-1}=\exp (-t X), \quad(\exp s X)(\exp t X)=\exp (s+t) X .
$$

For $z \in M$, we let $T_{z} M$ denote the complex tangent space of $M$ at $z$ and we define

$$
X(z):=\left.\left[\frac{d(z \exp t X)}{d t}\right]\right|_{t=0} \in T_{z} M .
$$

We identify $\mathfrak{X}$ with the Lie algebra $\mathfrak{g}$ for $M$ with the usual Lie bracket $[X, Y]$ for vector fields $X, Y \in \mathfrak{X}$. Note that $\operatorname{dim} \mathfrak{g}=n$. Finally, by the connectedness of $M$, given $g \in M$, there exists $t_{i} \in \mathbb{C}$ and $X_{i} \in \mathfrak{g}, i=$ $1, \ldots, N=N(g)$ such that $g=\prod_{i=1}^{N} \exp t_{i} X_{i}$; i.e.,

$$
M=\left\{\prod_{i=1}^{\nu} \exp t_{i} X_{i} \in M: \nu \in Z^{+}, t_{i} \in \mathbb{C}, X_{i} \in \mathfrak{g}\right\} .
$$

Given a Lie subalgebra $\mathfrak{g}_{0}$ of $\mathfrak{g}$ with $\operatorname{dim} \mathfrak{g}_{0}=d<n$, there is a corresponding connected complex Lie subgroup $H$, called the integral manifold of $\mathfrak{g}_{0}$, with the following properties: 
1. There is a holomorphic isomorphism from a neighborhood $V$ of $e$ in $H$ onto a neighborhood $U$ of 0 in $\mathfrak{g}_{0}$ whose inverse is given by the exponential map: $X \in U \mapsto g=\exp X \in V$.

2. $M$ is foliated by cosets $\{z H: z \in M\}$ of $H$; i.e., given $z_{0} \in M$, there exist local coordinates $\varphi: V \mapsto \Delta=\prod_{i=1}^{n}\left\{\left|w_{i}\right|<1\right\} \subset \mathbb{C}_{w}^{n}$ defined on a neighborhood $V$ of $z_{0}$ in $M$ with $\varphi\left(z_{0}\right)=0$ such that for each $\left|b_{j}\right|<$ $1, j=d+1, \ldots, n$, the $d$-dimensional polydisk $\left\{w \in \Delta: w_{j}=b_{j}, j=\right.$ $d+1, \ldots, n\}$ corresponds to the connected component of $(z H) \cap V$ where $z=\varphi^{-1}\left(0, \ldots, 0, b_{d+1}, \ldots, b_{n}\right)$. We call $(\varphi ; V, \Delta) \mathfrak{g}_{0}$-local coordinates at $z_{0}$ in $M$.

3. If $H$ is closed in $M$; i.e., if $H$ is a $d$-dimensional, non-singular, complex analytic set in $M$, the quotient space $M / H$ is an $(n-d)$-dimensional complex manifold with canonical projection

$$
\pi: z \in M \mapsto z H \in M / H
$$

We remark that $H$ is obtained as in (5.1) with $\mathfrak{g}_{0}$ replacing $\mathfrak{g}$. Note that in property 2., regardless of the size of the neighborhood $V$ of $z_{0}$, we may have $(z H \cap V) \cap\left(z^{\prime} H \cap V\right) \neq \emptyset$ for some $z^{\prime}=\varphi^{-1}\left(0, \ldots, 0, b_{d+1}^{\prime}, \ldots, b_{n}^{\prime}\right) \neq z$. If $H$ is closed in $M$, however, we can always find a neighborhood $V$ of $z_{0}$ with $(z H \cap V) \cap\left(z^{\prime} H \cap V\right)=\emptyset$ if $z \neq z^{\prime}$.

These three items concerning the subalgebra $\mathfrak{g}_{0}$ of $\mathfrak{g}$ form part of a holomorphic Frobenius theorem for complex Lie subgroups and subalgebras. Unless otherwise noted, all of our Lie subgroups will be complex Lie subgroups.

Let $D \Subset M$ be a pseudoconvex domain in $M$ with $C^{\infty}$ boundary. Fix $X \in \mathfrak{X} \backslash\{0\} ; \zeta \in D$ and

$$
B=\{t \in \mathbb{C}:|t|<\rho\}
$$

with $\rho>0$ sufficiently small so that $\zeta \exp t X \in D$ for $t \in B$. Given $w \in M$, let $R_{w}: M \rightarrow M$ via right multiplication by $w: R_{w}(z):=z w$. We consider the holomorphic map $T: B \times M \rightarrow B \times M$ defined as

$$
T(t, z)=(t, w):=(t, F(t, z)) \quad \text { where } F(t, z):=z(\zeta \exp t X)^{-1} .
$$

As in Corollary 4.2, we write $z=\phi(t, w)$ if $w=F(t, z)=z(\zeta \exp t X)^{-1}$ so that $\phi(t, w)=w(\zeta \exp t X)$. Let $\mathcal{D}=T(B \times D)$. Fixing a value of $t \in B$, we will write $D(t):=F(t, D)=D \cdot(\zeta \exp t X)^{-1}$. Note for $t \in B$ fixed,

$$
F(t, \cdot)=R_{(\zeta \exp t X)^{-1}} \in \operatorname{Aut}(M)
$$


Furthermore, for each $t \in B$, we have $e \in D(t)$ since, by hypothesis, $\zeta \exp t X \in$ $D$. Thus we can construct the $c$-Robin constant $\lambda(t)$ for $(D(t), e)$; and we have the following.

Lemma 5.1. Suppose

$$
\frac{\partial^{2} \lambda}{\partial t \partial \bar{t}}\left(t_{0}\right)=0
$$

for some $t_{0} \in B$. Then the integral curve $z \exp t X, t \in \mathbb{C}$, with initial value $z$, of the holomorphic vector field $X$ satisfies, for $A=D, \partial D$, or $\bar{D}^{c}$,

$$
\begin{aligned}
& \text { 1. } z \in A \text { implies } z \exp t X \in A \text { for all } t \in \mathbb{C} \text {; } \\
& \text { 2. } A \cdot z^{-1}=A \cdot(z \exp t X)^{-1} \text { for all } t \in \mathbb{C} \text { and all } z \in M \text {. }
\end{aligned}
$$

Proof. From the proof of Corollary 4.2 the nonvanishing holomorphic vector field $\Theta=\Theta\left(t_{0}, \cdot\right)$ on $M$ defined in (4.4) has the property that the entire integral curve $I\left(z_{0}\right)$ associated to $\Theta$ for any initial point $z_{0} \in \partial D$ lies on $\partial D$. We claim that $X=\Theta$. To verify the claim, write, in local coordinates,

$$
X=\sum_{k=1}^{n} \eta_{k} \frac{\partial}{\partial z_{k}}
$$

and

$$
\Theta=\sum_{k=1}^{n} \zeta_{k} \frac{\partial}{\partial z_{k}} .
$$

By left-invariance of $X$, for $z \in M$,

$$
\left.\left[\frac{d(z \exp t X)_{k}}{d t}\right]\right|_{t=t_{0}}=\eta_{k}\left(z \exp t_{0} X\right), \quad k=1, \ldots, n .
$$

On the other hand, by (4.4),

$$
\zeta_{k}(z)=\frac{\partial \phi_{k}}{\partial t}\left(t_{0}, F\left(t_{0}, z\right)\right)=\left.\left[\frac{\partial \phi_{k}(t, w)}{\partial t}\right]\right|_{t=t_{0}, w=z\left(\zeta \exp t_{0} X\right)^{-1}}
$$

But $z=\phi(t, w)=w(\zeta \exp t X)$; in particular,

$$
z_{k}=\phi_{k}(t, w)=(w \zeta \exp t X)_{k}, \quad k=1, \ldots, n .
$$

Hence, by (5.3)

$$
\begin{aligned}
\zeta_{k}(z) & =\left.\left[\frac{\partial \phi_{k}(t, w)}{\partial t}\right]\right|_{t=t_{0}, w=z\left(\zeta t_{0} X\right)^{-1}} \\
& =\left.\left[\eta_{k}\left(w \zeta \exp t_{0} X\right)\right]\right|_{w=z\left(\zeta \exp t_{0} X\right)^{-1}} \\
& =\eta_{k}(z)
\end{aligned}
$$


which proves $X=\Theta$.

By Corollary 4.2, this proves 1 . for the case $A=\partial D$. Using this result, as mentioned in 1. of Remark 4.1, 1. holds for $A=D$ and $A=\bar{D}^{c}$.

To prove 2., we first observe that

$$
z_{1} z_{2}^{-1}=\left[z_{1}(\exp t X)\right]\left[z_{2}(\exp t X)\right]^{-1}, \quad t \in \mathbb{C} .
$$

Take $A=\partial D$ and let $z \in M$ be fixed. For $z_{1} \in \partial D$, by 1 ., $z_{1}(\exp t X) \in \partial D$ for all $t \in \mathbb{C}$. Thus

$$
z_{1} z^{-1}=\left[z_{1}(\exp t X)\right][z(\exp t X)]^{-1} \in(\partial D) \cdot[z(\exp t X)]^{-1}
$$

so that $(\partial D) \cdot z^{-1} \subset(\partial D) \cdot[z(\exp t X)]^{-1}$. For the reverse inclusion, take $z_{1} \in \partial D$; then $z_{1}(\exp (-t X)) \in \partial D$ for all $t \in \mathbb{C}$ by 1 .; hence

$$
z_{1}[z(\exp t X)]^{-1}=z_{1}\left(\exp (-t X) z^{-1}\right) \in(\partial D) \cdot z^{-1}
$$

so that $(\partial D)[z(\exp t X)]^{-1} \subset(\partial D) \cdot z^{-1}$. Similar arguments show that 2 . holds for $A=D$ and $A=\bar{D}^{c}$.

We make another observation based on Lemma 5.1. From the proof, we see by using (5.2) that the holomorphic vector field $\Theta(t, z)$ on $M$ defined in (4.6) is independent of $t \in B$; indeed, it coincides with $X$. Suppose that $\frac{\partial^{2} \lambda}{\partial t \partial \bar{t}}\left(t_{0}\right)=0$ for some $t_{0} \in B$. From the necessity condition in Corollary 4.3, $X=\Theta\left(t_{0}, z\right)$ is thus a tangential vector field on $\partial D$. Fix $t \in B$. Since $\Theta(t, z)=X$, the suffciency condition of the corollary implies that $\frac{\partial^{2} \lambda}{\partial t \partial \bar{t}}(t)=$ 0 . From Theorem 4.1 we conclude that $D(t)=D(0)$ for $t \in B$. Thus, $D(\zeta \exp t X)^{-1}=D \zeta^{-1}$ for $t \in B$, and, aposteriori, $D(\zeta \exp t X)^{-1}=D \zeta^{-1}$ for all $t \in \mathbb{C}$.

We continue in the setting of a complex Lie group $M$ as before. Consider the following automorphism $T$ of $M \times M$ :

$$
T(z, w)=(z, W):=\left(z, w z^{-1}\right)=\left(z, R_{z^{-1}}(w)\right) .
$$

Let $D \Subset M$ be a domain with $C^{\infty}$ boundary and let

$$
\mathcal{D}:=T(D \times D)=\bigcup_{z \in D}(z, D(z))
$$

where

$$
D(z):=D \cdot z^{-1}=R_{z^{-1}}(D)=\left\{w z^{-1}: w \in D\right\} .
$$

This is a variation of domains $D(z)$ in $M$ with parameter space $D \subset M$. Note that $e \in D(z)$ for all $z \in D$ since, for $z \in D, z z^{-1} \in D(z)$. Let $G(z, W)$ be the $c$-Green function for $(D(z), e)$ and $\Lambda(z)$ the $c$-Robin constant. Then 
$\Lambda(z)$ is a $C^{\infty}$ function on $D$, called the $c$-Robin function for $D$. Finally, let $\left\{a_{k}(z)\right\}_{k=1, \ldots, n}$ be the eigenvalues (repeated with multiplicity) of the complex Hessian matrix $\left[\frac{\partial^{2}(-\Lambda)}{\partial \bar{z}_{j} \partial z_{k}}\right]_{j, k}$ at $z \in D$.

The following elementary result, which we state without proof, will be used repeatedly in this paper.

Proposition 5.1. Let $V$ be a domain in $\mathbb{C}^{n}$ and let $s(z)$ be a $C^{2}$ function on $V$. Assume that $\left.\left[\frac{\partial^{2} s\left(z_{0}+\mathbf{a} t\right)}{\partial t \partial \bar{t}}\right]\right|_{t=0}=0$ for some $z_{0} \in V$ and some $\mathbf{a} \in \mathbb{C}^{n} \backslash\{0\}$. Then for any holomorphic curve $z=\zeta(t),|t| \ll 1$ in $V$ with $\zeta(0)=z_{0}$ and $\left.\left[\frac{d \zeta(t)}{\partial t}\right]\right|_{t=0}=\mathbf{a}$, we have $\left.\left[\frac{\partial^{2} s(\zeta(t))}{\partial t \partial \bar{t}}\right]\right|_{t=0}=0$.

We use Lemma 5.1 to prove the following result, which will be crucial in all that follows.

Lemma 5.2. Suppose $D$ is pseudoconvex. Then $-\Lambda(z)$ is a plurisubharmonic exhaustion function for $D$. Furthermore, if $a_{i}(\zeta)=0$ for $i=1, \ldots, k \leq$ $n$ for some $\zeta \in D$, then there exist $k$ linearly independent holomorphic vector fields $X_{1}, \ldots, X_{k}$ in $\mathfrak{X}$ which satisfy the following conditions: For each $z \in D$ and each $X=X_{i}(i=1, \ldots, k)$,

$$
\begin{aligned}
\text { o. } & {\left.\left[\frac{\partial^{2} \Lambda(z \exp t X)}{\partial t \partial \bar{t}}\right]\right|_{t=0}=0 ; } \\
\text { i. } & z \exp t X \in D, t \in \mathbb{C} \text { and } D \cdot(z \exp t X)^{-1}=D \cdot z^{-1}, t \in \mathbb{C} ; \\
\text { ii. } & \Lambda(z \exp t X)=\Lambda(z), t \in \mathbb{C} ; \\
\text { iii. } & \{z \exp t X \in M: t \in \mathbb{C}\} \Subset D .
\end{aligned}
$$

Proof. We know that $-\Lambda$ is plurisubharmonic by Theorem 3.2 since $\mathcal{D}$ is pseudoconvex in $D \times M$. To show that $-\Lambda$ is an exhaustion function for $D$, since $D(z):=D \cdot z^{-1}$, if $z \rightarrow \eta \in \partial D$, then $\eta z^{-1} \in \partial D(z)$ and $\eta z^{-1} \rightarrow e$; hence we have $\Lambda(z) \rightarrow-\infty$ from standard potential theory arguments.

Now suppose $a_{i}(\zeta)=0$ for $i=1, \ldots, k \leq n$ for some $\zeta \in D$; i.e.,

$$
\left.\left[\frac{\partial^{2} \Lambda\left(\zeta+b_{i} t\right)}{\partial t \partial \bar{t}}\right]\right|_{t=0}=0, \quad i=1, \ldots, k
$$

for linearly independent vectors $b_{1}, \ldots, b_{k} \in \mathbb{C}^{n}$ (in local coordinates). We take any $X_{i} \in \mathfrak{X}$ such that

$$
X_{i}(\zeta)=\left.\left[\frac{d\left(\zeta \exp t X_{i}\right)}{d t}\right]\right|_{t=0}=b_{i} .
$$

Then $X_{1}, \ldots, X_{k}$ are linearly independent in $\mathfrak{X}$ and satisfy, by Proposition 5.1 .

$$
\left.\left[\frac{\partial^{2} \Lambda\left(\zeta \exp t X_{i}\right)}{\partial t \partial \bar{t}}\right]\right|_{t=0}=0
$$


For sufficiently small $|t|$, say for $t$ in $B=\{t:|t|<\rho\}$, we can assume that $\zeta \exp t X_{i} \subset D, i=1, \ldots, k$. Consider the transformation

$$
T_{i}(t, z)=(t, w)=\left(t, F_{i}(t, z)\right)
$$

where $w=F_{i}(t, z)=z\left(\zeta \exp t X_{i}\right)^{-1}$. Then $\mathcal{D}_{i}:=T_{i}(B \times D)$ defines a variation of domains $D_{i}(t):=F_{i}(t, D)=\left\{z\left(\zeta \exp t X_{i}\right)^{-1} \in M: z \in D\right\}=$ $D \cdot\left(\zeta \exp t X_{i}\right)^{-1}$; moreover, $e \in D_{i}(t), t \in B$. Let $g_{i}(t, w)$ be the $c$-Green function for $\left(D_{i}(t), e\right)$ and let $\lambda_{i}(t)$ be the $c$-Robin constant for $\left(D_{i}(t), e\right)$. By definition, we have $\lambda_{i}(t)=\Lambda\left(\zeta \exp t X_{i}\right)$, so that

$$
\frac{\partial^{2} \lambda_{i}}{\partial t \partial \bar{t}}(0)=\left.\left[\frac{\partial^{2} \Lambda\left(\zeta \exp t X_{i}\right)}{\partial t \partial \bar{t}}\right]\right|_{t=0}=0, \quad i=1, \ldots, k .
$$

We now make direct use of the previous result for $A=D$ to conclude that $z \in D$ if and only if $z \exp t X_{i} \in D$ for all $t \in \mathbb{C}$; also $D \cdot z^{-1}=D \cdot\left(z \exp t X_{i}\right)^{-1}$ for all $t \in \mathbb{C}$, so that i. is proved. Assertion i. and the definition of $\Lambda(z)$ imply ii. Furthermore, since $-\Lambda$ is an exhaustion function for $D$, for a fixed $z \in D$ the level set $S_{z}:=\left\{z^{\prime} \in D: \Lambda\left(z^{\prime}\right)=\Lambda(z)\right\}$ is relatively compact in $D$. Together with ii. this implies iii. Finally, since $-\Lambda$ is a plurisubharmonic exhaustion function for $D,-\Lambda$ is subharmonic and bounded on the complex curve $\{z \exp t X, t \in \mathbb{C}\}$. This curve is conformally equivalent to $\mathbb{C}, \mathbb{C} \backslash\{0\}$ or a one-dimensional compex torus and hence $-\Lambda$ is constant on this curve, which implies $O$.

Remark 5.1. Condition $i$. for $X \in \mathfrak{X}$ is clearly equivalent to

$$
D \cdot \exp t X=D, t \in \mathbb{C}
$$

i.e., $D$ is invariant under the one parameter transformation group $\exp t X$ of $M$. Indeed, the proof of the lemma shows that $o$. $-i i i$. for $X \in \mathfrak{X}$ are all equivalent; and hence equivalent to (5.4).

Corollary 5.1. Suppose $D \Subset M$ is pseudoconvex with smooth boundary. Then $-\Lambda(z)$ is not strictly plurisubharmonic in $D$ if and only if there exists $X \in \mathfrak{X} \backslash\{0\}$ for which one of the four equivalent conditions o. $\sim$ iii. in Lemma 5.2 hold. Moreover, for such $X,\{z \exp t X \in M: t \in C\} \Subset A$ for $z \in A$ where $A=D, \partial D$ or $\bar{D}^{c}$.

Proof. We have already verified the first part; and iii. verifies the last statement for $A=D$. To prove the last part for $A=\partial D$ or $\bar{D}^{c}$, from Lemma 5.1] we have $z \in A$ if and only if $z \exp t X \in A$ for all $t \in \mathbb{C}$; and 
$A \cdot z^{-1}=A \cdot(z \exp t X)^{-1}$. Since $\partial D$ is compact, this verifies the corollary for $A=\partial D$. To verify the corollary for $A=\bar{D}^{c}$, fix $z \in \bar{D}^{c}$ and $z_{0} \in \partial D$. Then

$$
z \exp t X=z z_{0}^{-1}\left(z_{0} \exp t X\right)
$$

and $\left\{z_{0} \exp t X: t \in \mathbb{C}\right\} \Subset \partial D$ since $z_{0} \in \partial D$ so that $\{z \exp t X: t \in \mathbb{C}\} \Subset M$. Thus it suffices to show that

$$
\overline{z \exp t X} \cap \partial D=\emptyset .
$$

If (5.5) does not hold, there exists a point $\eta \in \partial D$ and a sequence $\left\{t_{\nu}\right\}_{\nu}$ in $\mathbb{C}$ such that $z\left(\exp t_{\nu} X\right) \rightarrow \eta$ as $\nu \rightarrow \infty$. We write $D_{1}(z):=\bar{D}^{c} \cdot z^{-1}$ for $z \in \bar{D}^{c}$. Then $e \in D_{1}(z)$ for such $z$, so that we can consider the $c$-Green function $G_{1}(z, \cdot)$ and the $c$-Robin constant $\Lambda_{1}(z)$ for $\left(D_{1}(z), e\right)$. (Note that although $D_{1}(z)$ is not necessarily relatively compact in $M$, we can define the $c$-Green function $G_{1}(z, \cdot)$ and the $c$-Robin constant $\Lambda_{1}(z)$ for $\left(D_{1}(z), e\right)$ by a standard exhaustion method.) If $Z \in \bar{D}^{c} \rightarrow \eta \in \partial D$, then $\eta Z^{-1} \in$ $\partial D_{1}(Z)$ and $\eta Z^{-1} \rightarrow e$; thus $\Lambda_{1}(Z) \rightarrow-\infty$ as $Z \rightarrow \eta$. In particular, $\Lambda_{1}\left(z\left(\exp t_{\nu} X\right)\right) \rightarrow-\infty$ as $\nu \rightarrow \infty$. On the other hand, from the previous results, $D_{1}(z)=D_{1}\left(z \exp t_{\nu} X\right), \nu=1,2, \ldots$ Thus, $\Lambda_{1}(z)=\Lambda_{1}\left(z \exp t_{\nu} X\right)$ for all $\nu$, and since $\Lambda_{1}(z)>-\infty$, we obtain a contradiction, verifying (5.5).

Corollary 5.2. Suppose $D \Subset M$ is pseudoconvex with $C^{\infty}$ boundary. Let $c>0$ be a $C^{\infty}$ function on $M$ and let $\Lambda(z)$ be the c-Robin function for D. If the complex Hessian matrix $\left[\frac{\partial^{2}(-\Lambda)}{\partial z_{j} \partial \bar{z}_{k}}(\zeta)\right]$ has a zero eigenvalue with multiplicity $k \geq 1$ at some point $\zeta \in D$, then the complex Hessian matrix of any plurisubharmonic exhaustion function $s(z)$ for $D$ has a zero eigenvalue with multiplicity at least $k$ at each point $z \in D$.

Proof. Take $X_{i} \in \mathfrak{X} \backslash\{0\}, i=1, \ldots, k$ as in the lemma. Given $z \in D$, $\left\{z \exp t X_{i}: t \in \mathbb{C}\right\} \Subset D$. Thus if $s$ is any plurisubharmonic exhaustion function for $D, s$ is subharmonic and bounded on this complex curve and hence, as in the proof of Corollary [5.1, $s$ is constant on the curve. Thus $\left[\frac{\partial^{2} s}{\partial z_{a} \partial \bar{z}_{b}}(z)\right]$ has a zero eigenvalue of multiplicity at least $k$ (cf., [17] Proposition 2.1). This is valid for each $z \in D$.

We remark that the conclusion of the corollary implies, in particular, that $D$ is not Stein. We now address the following question: for a complex Lie group $M$, when is a pseudoconvex domain $D \Subset M$ with $C^{\infty}$ boundary Stein? An answer is provided in the following result.

Theorem 5.1. Let $D \Subset M$ be a pseudoconvex domain with smooth boundary which is not Stein. Then 
I. there exists a unique connected complex Lie subgroup $H$ of $M$ such that

1. $\operatorname{dim} H \geq 1$;

2. $D$ is foliated by cosets of $H ; D=\bigcup_{z \in D} z H$ with $z H \Subset D$;

3. any holomorphic curve $\ell:=\{z=z(t) \in D: t \in \mathbb{C}\}$ with $\ell \Subset D$ is necessarily contained in some coset $z H$ in $D$.

II. Furthermore, if $H$ is closed in $M$ and $\pi: M \mapsto M / H$ is the canonical projection, then $H$ is a complex torus and there exists a Stein domain $D_{0} \Subset M / H$ with smooth boundary such that $D=\pi^{-1}\left(D_{0}\right)$.

Proof. Let $\Lambda(z)$ denote the $c$-Robin function on $D$; this is a $C^{\infty}$ plurisubharmonic exhaustion function on $D$. We utilize $\Lambda(z)$ to define a Lie subalgebra $\mathfrak{g}_{0}$ of $\mathfrak{g}$ and a candidate Lie subroup $H$ using (5.1) with $\mathfrak{g}_{0}$ replacing $\mathfrak{g}$. We begin with the construction of $\mathfrak{g}_{0}$, then of $H$; finally we verify the properties of $H$. We divide this procedure into several steps.

$1^{\text {st }}$ step. Let $\zeta \in D$ and define

$$
\mathfrak{X}_{\zeta}:=\left\{X \in \mathfrak{X}:\left.\left[\frac{\partial^{2} \Lambda(\zeta \exp t X)}{\partial t \partial \bar{t}}\right]\right|_{t=0}=0\right\} .
$$

Then $\mathfrak{X}_{\zeta}$ is independent of $\zeta \in D$ and $\operatorname{dim} \mathfrak{X}_{\zeta}=d \geq 1$.

Indeed, since $D$ is not Stein, $-\Lambda(z)$ is not strictly plurisubharmonic on $D$; i.e., in local coordinates there exists a point $\zeta \in D$ and $b \in \mathbb{C}^{n} \backslash\{0\}$ such that $\left.\left[\frac{\partial^{2} \Lambda(\zeta+b t)}{\partial t \partial \bar{t}}\right]\right|_{t=0}=0$. The $1^{\text {st }}$ step now follows from Lemma $[5.2$ and Corollary 5.1.

Thus we write $\mathfrak{g}_{0}:=\mathfrak{X}_{\zeta}$. We will show in the $3^{\text {rd }}$ step that $\mathfrak{g}_{0}$ is, indeed, a Lie subalgebra of $\mathfrak{g}$.

$2^{\text {nd }}$ step. Fix $\zeta \in D$ and an integer $m \geq 1$. Then

$$
\Lambda\left(\zeta \prod_{k=1}^{m} \exp t_{k} X_{k}\right)=\Lambda(\zeta), \text { for all } t_{k} \in \mathbb{C}, X_{k} \in \mathfrak{g}_{0} .
$$

To see this, from Corollary 5.1 and (5.4) we have

$$
D \cdot\left(\exp t_{m} X_{m}\right)^{-1}=D, \quad \text { for all } t_{m} \in \mathbb{C}, X_{m} \in \mathfrak{g}_{0} .
$$

Thus for $t_{m-1} \in \mathbb{C}$,

$$
\begin{aligned}
D \cdot\left(\exp t_{m-1} X_{m-1} \exp t_{m} X_{m}\right)^{-1} & =D \cdot\left(\exp t_{m} X_{m}\right)^{-1}\left(\exp t_{m-1} X_{m-1}\right)^{-1} \\
& =D \cdot\left(\exp t_{m-1} X_{m-1}\right)^{-1} \\
& =D
\end{aligned}
$$


Inductively, we have

$$
D \cdot\left[\prod_{k=1}^{m} \exp t_{k} X_{k}\right]^{-1}=D, \quad \text { for all } t_{k} \in \mathbb{C}, X_{k} \in \mathfrak{X}_{0} .
$$

This is equivalent to

$$
D \cdot\left[\zeta \prod_{k=1}^{m} \exp t_{k} X_{k}\right]^{-1}=D \cdot \zeta^{-1}, \quad \text { for all } t_{k} \in \mathbb{C}, X_{k} \in \mathfrak{g}_{0} .
$$

The $2^{\text {nd }}$ step now follows immediately from the definition of $\Lambda(z)$.

$3^{\text {rd }}$ step. The space $\mathfrak{g}_{0}$ is a Lie subalgebra of $\mathfrak{g}$; i.e., $\mathfrak{g}_{0}$ is a linear subspace of $\mathfrak{g}$ and $X, Y \in \mathfrak{g}_{0}$ implies $[X, Y] \in \mathfrak{g}_{0}$.

To prove that $\mathfrak{g}_{0}$ is a linear subspace of $\mathfrak{g}$, fix $\zeta \in D$; let $X, Y \in \mathfrak{g}_{0}$; and let $\alpha, \beta \in \mathbb{C}$. Since it is clear that $\alpha X, \beta Y \in \mathfrak{g}_{0}$, from the $2^{\text {nd }}$ step we have

$$
\Lambda(\zeta \exp (\alpha t) X \exp (\beta t) Y)=\Lambda(\zeta), \quad \text { for all } t \in \mathbb{C} .
$$

Thus

$$
\left.\left[\frac{\partial^{2} \Lambda(\zeta \exp (\alpha t) X \exp (\beta t) Y)}{\partial t \partial \bar{t}}\right]\right|_{t=0}=0
$$

It is a standard result that for any $X, Y \in \mathfrak{g}$

$$
\exp (\alpha t) X \exp (\beta t) Y=\exp \left[t(\alpha X+\beta Y)+O\left(t^{2}\right)\right]
$$

near $t=0$ and hence it follows that

$$
\left.\left[\frac{d(\zeta \exp t(\alpha X+\beta Y))}{d t}\right]\right|_{t=0}=\left.\left[\frac{d(\zeta \exp (\alpha t) X \exp (\beta t) Y)}{d t}\right]\right|_{t=0} .
$$

Using Proposition 5.1 we see that $\alpha X+\beta Y \in \mathfrak{g}_{0}$; i.e.,

$$
\left.\left[\frac{\partial^{2} \Lambda(\zeta \exp t(\alpha X+\beta Y))}{\partial t \partial \bar{t}}\right]\right|_{t=0}=\left.\left[\frac{\partial^{2} \Lambda(\zeta(\exp (\alpha t) X \exp (\beta t) Y))}{\partial t \partial \bar{t}}\right]\right|_{t=0}=0 .
$$

To prove that $X, Y \in \mathfrak{g}_{0}$ implies $[X, Y] \in \mathfrak{g}_{0}$, fix $\zeta \in D$. From the $2^{\text {nd }}$ step we have

$$
\Lambda(\zeta \exp (-\sqrt{t} X) \exp (-\sqrt{t} Y) \exp \sqrt{t} X \exp \sqrt{t} Y)=\Lambda(\zeta), \quad \text { for all } t \in \mathbb{C} .
$$

On the other hand, it is known that

$$
\left.\exp (-\sqrt{t} X) \exp (-\sqrt{t} Y) \exp \sqrt{t} X \exp \sqrt{t} Y=\exp (t[X, Y])+O\left(t^{3 / 2}\right)\right)
$$


near $t=0$ so that

$\left.\left[\frac{d(\zeta \exp (-\sqrt{t} X) \exp (-\sqrt{t} Y) \exp \sqrt{t} X \exp \sqrt{t} Y)}{d t}\right]\right|_{t=0}=\left.\left[\frac{d(\zeta \exp t[X, Y])}{d t}\right]\right|_{t=0}$.

It follows from Proposition 5.1 that

$$
\begin{aligned}
& {\left.\left[\frac{\partial^{2} \Lambda(\zeta \exp (t[X, Y])}{\partial t \partial \bar{t}}\right]\right|_{t=0} } \\
= & {\left.\left[\frac{\partial^{2} \Lambda(\zeta \exp (-\sqrt{t} X) \exp (-\sqrt{t} Y) \exp \sqrt{t} X \exp \sqrt{t} Y)}{\partial t \partial \bar{t}}\right]\right|_{t=0} } \\
= & {\left.\left[\frac{\partial^{2} \Lambda(\zeta)}{\partial t \partial \bar{t}}\right]\right|_{t=0}=0 . }
\end{aligned}
$$

Since $[X, Y] \in \mathfrak{X}$, we see from Lemma 5.2 and Corollary 5.1 that $[X, Y] \in \mathfrak{g}_{0}$.

From the Frobenius theorem described at the beginning of this section and the fact that $\mathfrak{g}_{0}$ is a Lie subalgebra of $\mathfrak{g}$, we have the integral manifold $H$ in $M$ for $\mathfrak{g}_{0}$ passing through the identity $e$, i.e., from (5.1),

$$
H=\left\{\prod_{i=1}^{\nu} \exp t_{i} X_{i} \in M: \nu \in Z^{+}, t_{i} \in \mathbb{C}, X_{i} \in \mathfrak{g}_{0}\right\} .
$$

$4^{\text {th }}$ step. $H$ satisfies conditions 1., 2. and 3. in Theorem 5.1.

Since $\operatorname{dim} H=\operatorname{dim} \mathfrak{g}_{0} \geq 1, H$ satisfies 1 . Fix $\zeta \in D$. We consider the level set

$$
S_{\zeta}:=\{z \in D: \Lambda(z)=\Lambda(\zeta)\} .
$$

Since $-\Lambda$ is an exhaustion function for $D$, we have $S_{\zeta} \Subset D$. Fix $X \in \mathfrak{g}_{0}$. We have

$$
\Lambda(\zeta \exp t X)=\Lambda(\zeta), \quad \text { for all } t \in \mathbb{C}
$$

so that $\zeta \exp t X \subset S_{\zeta}$. Since $t \in \mathbb{C}$ and $X \in \mathfrak{g}_{0}$ were arbitrary, it follows from (5.10) that we have $\Lambda(\zeta g)=\Lambda(\zeta)$ for all $g \in H$. Hence $\zeta H \subset S_{\zeta} \Subset D$. The conclusion that $D$ has a foliation $D=\cup_{z \in D} z H$ follows from this observation together with the fact that $M$ itself is foliated by cosets $M=\cup_{z \in M} z H$ (the second item of the Frobenius theorem). Thus $H$ satisfies 2.

We shall prove that $H$ satisfies 3 . by contradiction. Thus we assume that there exists a holomorphic curve $\ell:=\{z=z(t) \in D: t \in \mathbb{C}\}$ with $\ell \Subset D$ and with the property that there exists a coset $z_{0} H$ in $D$ with $\ell \cap z_{0} H \neq \emptyset$ and $\ell \not \subset z_{0} H$. Since $D$ is foliated by cosets $z H$, we may assume that $\ell$ and $z_{0} H$ intersect transversally at some point $\zeta$ in $D$. Let $a \in \mathbb{C} \backslash\{0\}$ be a tangent vector of $\ell$ at $\zeta$ and choose $X \in \mathfrak{X}$ such that $X(\zeta)=a$. By assumption $\Lambda=$ const. $=\Lambda(\zeta)$ on $\ell$. It follows from Lemma 5.1 that $X \in \mathfrak{g}_{0}$, so 
that $C:=\{\zeta \exp t X: t \in \mathbb{C}\} \subset \zeta H$. However, the curve $C$ intersects $z_{0} H$ transversally; in particular, $C \not \subset z_{0} H$. From property 2., since $z_{0} H \cap \zeta H \neq \emptyset$, we have $z_{0} H=\zeta H$, which yields a contradiction.

$5^{\text {th }}$ step. $H$ satisfying 1., 2. and 3. is uniquely determined.

Let $\widehat{H}$ be another Lie subgroup of $M$ which satisfies 1., 2. and 3. in the theorem. We write $\widehat{\mathfrak{g}}_{0}$ for the connected Lie subalgebra of $\mathfrak{g}$ which corresponds to $\widehat{H}$. Thus from (5.1),

$$
\widehat{H}=\left\{\prod_{i=1}^{\nu} \exp t_{i} X_{i} \in M: \nu \in \boldsymbol{Z}^{+}, t_{i} \in \mathbb{C}, \widehat{X}_{i} \in \widehat{\mathfrak{g}}_{0}\right\} .
$$

Fix $\widehat{X} \in \widehat{\mathfrak{g}}_{0}$ and $\zeta \in D$. Since $\zeta \widehat{H} \Subset D$, it follows from condition 3. for $H$ that the analytic curve $\{\zeta \exp t \widehat{X}, t \in \mathbb{C}\}$ passing through $\zeta$ is contained in $\zeta H$. By the above description of $\widehat{H}$ we inductively have $\zeta \widehat{H} \subset \zeta H$, so that $\widehat{H} \subset H$. The converse inclusion is proved in a similar fashion.

$6^{\text {th }}$ step. Assertion II is true.

Suppose $H$ is closed in $M$. By 2. $H$ is compact in $M$, so that $H$ is a compact complex Lie group, i.e., a complex torus. Let $\pi: M \mapsto M / H$ be the canonical projection. We set $D_{0}:=\pi(D) \subset M / H$. Since $D$ is foliated by cosets of $H$ (property 2.), $\pi^{-1}\left(D_{0}\right)=D$; moreover, $\pi^{-1}\left(\partial D_{0}\right)=\partial D$, and since $\partial D$ is smooth in $M$ we have $\partial D_{0}$ is smooth in $M / H$. Given $\xi \in D_{0}$, we choose a point $\zeta \in \pi^{-1}(\xi) \subset D$ and define

$$
\Lambda_{0}(\xi):=\Lambda(\zeta)
$$

Again from property 2., this does not depend on the choice of $\zeta \in D$ with $\pi(\zeta)=\xi$; i.e., $\Lambda_{0}$ is a well-defined function on $D_{0}$. We show that $\Lambda_{0}$ is a $C^{\infty}$ strictly plurisubharmonic exhaustion function for $D_{0}$.

To this end, let $\xi_{n} \in D_{0} \rightarrow \xi \in \partial D_{0} \subset M / H$ as $n \rightarrow \infty$. We can choose $\zeta_{n} \in D$ and $\zeta \in \partial D$ with $\pi\left(\zeta_{n}\right)=\xi_{n}$ and $\pi(\zeta)=\xi$ in such a way that $\zeta_{n} \rightarrow \zeta$ in $M$ as $n \rightarrow \infty$. Since $-\Lambda$ is an exhaustion function for $D$, it follows that $-\Lambda_{0}\left(\xi_{n}\right)=-\Lambda\left(\zeta_{n}\right) \rightarrow \infty$, so that $-\Lambda_{0}$ is an exhaustion function for $D_{0}$. Next, fix $\xi_{0} \in D_{0}$ and take a point $\zeta_{0} \in D$ with $\pi^{-1}\left(\zeta_{0}\right)=\xi_{0}$. From 2 . of the Frobenius theorem we have $\mathfrak{g}_{0}$-local coordinates $\varphi: V \Subset D \mapsto \Delta=$ $\prod_{i=1}^{n}\left\{\left|w_{i}\right|<1\right\}$ with $\varphi\left(\zeta_{0}\right)=0$. We write

$$
w=\left(w_{1}, \ldots, w_{d} ; w_{d+1}, \ldots, w_{n}\right)=\left(w^{\prime} ; w^{\prime \prime}\right) \in \Delta^{\prime} \times \Delta^{\prime \prime}=\Delta
$$

where $\Delta^{\prime}=\prod_{i=1}^{d}\left\{\left|w_{i}\right|<1\right\}$ and $\Delta^{\prime \prime}=\prod_{i=d+1}^{n}\left\{\left|w_{i}\right|<1\right\}$; we also write $0=\left(0^{\prime} ; 0^{\prime \prime}\right) \in \Delta^{\prime} \times \Delta^{\prime \prime}$. We define, for $w^{\prime \prime} \in \Delta^{\prime \prime}, s\left(w^{\prime \prime}\right):=\varphi^{-1}\left(0^{\prime} ; w^{\prime \prime}\right) \subset V$. Then, since $H$ is closed in $M, V=\cup_{w^{\prime \prime} \in \Delta^{\prime \prime}}\left(s\left(w^{\prime \prime}\right) H\right) \cap V$ and this is a disjoint union (if necessary, take a smaller neighborhood $V$ of $\zeta_{0}$ ). Setting 
$\pi(V)=\delta \subset D_{0}$, for any $\xi \in \delta$ there exists a unique point $w^{\prime \prime} \in \Delta^{\prime \prime}$ such that $\pi\left(s\left(w^{\prime \prime}\right)\right)=\xi$. Consequently,

$$
\varphi_{0}: \xi \in \delta \mapsto w^{\prime \prime} \in \Delta^{\prime \prime}
$$

gives local coordinates for the neighborhood $\delta$ of $\xi_{0}$ in $D_{0}$. Identifying $s\left(w^{\prime \prime}\right)$ with $\left(0^{\prime} ; w^{\prime \prime}\right)$ via the mapping $\varphi$ and identifying $\xi$ with $w^{\prime \prime}$ via the mapping $\varphi_{0}$, from the definition of $\Lambda_{0}$ for $w^{\prime \prime}=\left(w_{d+1}, \ldots, w_{n}\right) \in \Delta^{\prime \prime}$ we have

$$
\Lambda_{0}\left(w_{d+1}, \ldots, w_{n}\right)=\Lambda\left(0, \ldots, 0 ; w_{d+1}, \ldots, w_{n}\right) .
$$

Since $-\Lambda$ is plurisubharmonic on $\Delta$, it follows that $-\Lambda_{0}$ is plurisubharmonic on $\Delta^{\prime \prime}$, and hence $-\Lambda_{0}$ is plurisubharmonic on $D_{0}$. It remains to verify that $-\Lambda_{0}$ is strictly plurisubharmonic on $D_{0}$. If not, there exists a point $\xi_{0} \in D_{0}$ at which $-\Lambda_{0}$ is not strictly plurisubharmonic. From formula (5.11) $-\Lambda_{0}\left(w_{d+1}, \ldots, w_{n}\right)$ is not strictly plurisubharmonic at the origin $0^{\prime \prime}$. Thus $-\Lambda\left(0, \ldots, 0 ; w_{d+1}, \ldots, w_{n}\right)$ is not strictly plurisubharmonic at $w^{\prime \prime}=0^{\prime \prime}$. This means that there exists $\left(b_{d+1}, \ldots, b_{n}\right) \in \mathbb{C}^{n-d} \backslash\left\{0^{\prime \prime}\right\}$ such that

$$
\left.\left[\frac{\partial^{2} \Lambda\left(0, \ldots, 0 ; b_{d+1} t, \ldots, b_{n} t\right)}{\partial t \partial \bar{t}}\right]\right|_{t=0}=0
$$

On the other hand, from the previous steps we know that $\Lambda\left(w^{\prime} ; 0^{\prime \prime}\right) \equiv \Lambda\left(0^{\prime} ; 0^{\prime \prime}\right)$ on $\Delta^{\prime}$; thus it follows that there exist at least $d+1$ linearly independent eigenvectors with eigenvalue 0 for the matrix $\left[\frac{\partial^{2}(-\Lambda)}{\partial z_{i} \partial \bar{z}_{j}}\right]_{i, j=1, \ldots, n}$ at $\zeta_{0}=s\left(0^{\prime \prime}\right) \in$ $D$. This contradicts the definition of the dimension $d$ of $\mathfrak{g}_{0}$.

Remark 5.2. In Theorem 5.1 the complex Lie group $M$ is a real Lie group of dimension $2 n$ with real Lie algebra $\mathfrak{g}$. If $H$ is not closed in $M$ the closure $\bar{H}$ of $H$ in $M$ is a closed real Lie subgroup of $M$ whose real dimension $m$ is less than $2 n$. In this case, we have the real Lie subalgebra $\mathfrak{g}_{0} \subset \mathfrak{g}$ corresponding to $\bar{H}$, and the projection $\widetilde{\pi}: M \rightarrow M / \bar{H}$, where $M / \bar{H}$ is a real manifold of dimension $2 n-m$. From properties 1 . and 2., $\bar{H}$ is a compact real submanifold in $M$ with $\bar{H} \subset D$ and $D$ is foliated by cosets of $\bar{H}$, so that $\Lambda=$ const. on each $z \bar{H}, z \in D$. Furthermore, $D_{0}:=\widetilde{\pi}(D)$ is a relatively compact domain with smooth boundary in $M / \bar{H}$ and $D=\widetilde{\pi}^{-1}\left(D_{0}\right)$.

Corollary 5.3. The subgroup $H$ of $M$ in Theorem 5.1 is normal.

Proof. We may assume that $D$ contains the identity, e. For if $z_{0} \in D$ and we define $D_{1}:=z_{0}^{-1} D$, then $D$ is a pseudoconvex domain with smooth boundary; $D_{1}$ is not Stein; and $e \in D_{1}$. Let $\Lambda_{1}$ be the $c$-Robin function for $D_{1}$. Assume that $X \in \mathfrak{X}$ satisfies

$$
\left.\left[\frac{\partial^{2} \Lambda\left(z_{0} \exp t X\right)}{\partial t \partial \bar{t}}\right]\right|_{t=0}=0
$$


Then by Corollary 5.2 we have $C:=\left\{z_{0} \exp t X \in M: t \in \mathbb{C}\right\} \Subset D$. We thus have $C_{1}:=\{\exp t X \in M: t \in \mathbb{C}\} \Subset z_{0}^{-1} D=D_{1}$, so that $\Lambda_{1}=$ const. on $C_{1}$. Thus,

$$
\left.\left[\frac{\partial^{2} \Lambda_{1}(\exp t X)}{\partial t \partial \bar{t}}\right]\right|_{t=0}=0 .
$$

A similar argument shows that $(* *)$ implies $(*)$. It follows that the Lie subroup $H_{1}$ in Theorem 5.1 corresponding to $D_{1}$ coincides with $H$. Thus we may indeed assume that $e \in D$.

We consider the isomorphism $\widetilde{T}(\zeta, z)$ of $M$ for $z \in M$ given by left multiplication by $z^{-1}$ :

$$
\widetilde{T}(\zeta, z): \zeta \in M \mapsto w=z^{-1} \zeta \in M
$$

This induces the variation of domains $\widetilde{D}(z)$ in $M$ :

$$
\widetilde{\mathcal{D}}: z \in D \mapsto \widetilde{D}(z)=z^{-1} D .
$$

Since each $\widetilde{D}(z), z \in D$, contains the identity element $e$, we can form the $c$-Robin function $\tilde{\lambda}(z)$ on $D$. We let $\widetilde{\mathfrak{g}}$ denote the Lie algebra consisting of all right-invariant holomorphic vector fields on $M$. For a fixed $\zeta \in D$ we define

$$
\widetilde{\mathfrak{g}}_{0}:=\left\{\widetilde{X} \in \widetilde{\mathfrak{g}}:\left.\left[\frac{\partial^{2} \widetilde{\lambda}((\exp t \tilde{X}) \zeta)}{\partial t \partial \bar{t}}\right]\right|_{t=0}=0\right\}
$$

As with $\mathfrak{g}_{0}$, the set $\widetilde{\mathfrak{g}}_{0}$ is a Lie subalgebra of $\widetilde{\mathfrak{g}}$. We construct the connected Lie subgroup $\widetilde{H}$ corresponding to $\widetilde{\mathfrak{g}}_{0}$ :

$$
\widetilde{H}:=\left\{\prod_{i=1}^{\nu} \exp t_{i} X_{i} \in M: \nu \in Z^{+}, t_{i} \in \mathbb{C}, X_{i} \in \widetilde{\mathfrak{g}}_{0}\right\}
$$

with $\operatorname{dim} \widetilde{H}=\widetilde{d} \geq 1$ and satisying the corresponding properties $1 ., 2$. and 3 . in Theorem 5.1. We first prove that

$$
z H=\widetilde{H} z, \quad \text { for any } z \in D .
$$

To prove this, fix $z \in D$ and $p \in \widetilde{H} z$ with $p=\left(\prod_{i=1}^{\nu} \exp t_{i} \widetilde{X}_{i}\right) z$ for some $t_{i} \in \mathbb{C}$ and $\widetilde{X}_{i} \in \widetilde{H}$. Condition 2. for $\widetilde{H}$ implies that the analytic curve $C_{\nu}:=\left\{\left(\exp t \widetilde{X}_{\nu}\right) z: t \in \mathbb{C}\right\}$ is relatively compact in $D$. Condition 3 . for $H$ thus implies that $C_{\nu} \Subset z H$. In paricular, $z_{\nu}:=\left(\exp t_{\nu} \widetilde{X}_{\nu}\right) z \in z H$ and hence $z_{\nu} H \subset z H$. If we consider the analytic curve $C_{\nu-1}:=\left\{\left(\exp t \widetilde{X}_{\nu-1}\right) z_{\nu} \in\right.$ $M: t \in \mathbb{C}\}$, then a similar argument shows that $C_{\nu-1} \Subset z_{1} H$, so that $z_{\nu-1}:=\left(\exp t_{\nu-1} \widetilde{X}_{\nu-1}\right) z_{\nu} \in z_{\nu} H \subset z H$, and hence $z_{\nu-1} H \subset z H$. Repeating this argument we obtain $\left(\exp t_{1} \widetilde{X}_{1}\right) z_{2} \in z_{2} H \subset z H$, i.e., $p \in z H$. We thus 
have $\widetilde{H} z \subset z H$. The reverse inclusion is proved similarly and equality (5.12) holds for $z \in D$.

Since $e \in D$ it follows that $\widetilde{H}=H$. Thus $z H=H z$ for $z \in D$. To extend this equality to all $z \in M$, we consider

$$
\mathcal{A}:=\left\{z \in M: z^{-1} H z=H\right\} \subset M .
$$

Then $D \subset \mathcal{A}$ and we want to show that $\mathcal{A}=M$. Clearly $H \subset \mathcal{A}$ and $\mathcal{A}$ is a subgroup of $M$. Since $e \in D \subset \mathcal{A}$, we can choose a neighborhood $V \subset \mathcal{A}$ of $e$. We utilize this neighborhood $V$ to show that $\mathcal{A}$ is open and closed in $M$. To show that $\mathcal{A}$ is open, fix $z_{0} \in \mathcal{A}$. Then $V z_{0}$ is a neighborhood of $z_{0}$ in $M$. Since $V \subset \mathcal{A}$, it follows that $V z_{0} \subset \mathcal{A}$ (since $\mathcal{A}$ is a group), and hence $\mathcal{A}$ is open. To show that $\mathcal{A}$ is closed, fix a point $\zeta \in \partial \mathcal{A}$. Choose $z \in \mathcal{A}$ sufficiently close to $\zeta$ so that $a:=z^{-1} \zeta \in V \subset \mathcal{A}$. Hence $\zeta=z a \in \mathcal{A}$, and $\mathcal{A}$ is closed.

We shall give two concrete examples of complex Lie groups $M$ in Theorem 5.1 or Remark 5.2.

1. Consider

$$
M=\text { Aut } \mathbb{C}=\{a z+b: a \in \mathbb{C} \backslash\{0\}, b \in \mathbb{C}\} .
$$

This is a complex Lie group of complex dimension 2 with the group operation of composition. This composition gives a group multiplication on $\mathbb{C} \backslash\{0\} \times \mathbb{C}$ which is noncommutative: $(a, b) \cdot(c, d)=(a c, a d+b)$. That is, if $f(z)=a z+b$ and $g(z)=c z+d$, then

$$
(f \circ g)(z)=(a c) z+(a d+b) .
$$

Note that any left-invariant holomorphic vector field on $M$ is of the form

$$
X=\alpha X_{1}+\beta X_{2}, \quad \alpha, \beta \in \mathbb{C},
$$

where

$$
X_{1}=a \frac{\partial}{\partial a}, \quad X_{2}=a \frac{\partial}{\partial b} .
$$

We have

$$
\exp t X_{1}=\left(e^{t}, 0\right) \quad \text { and } \quad \exp t X_{2}=(1, t) .
$$

Hence, $\exp t X=\left(e^{\alpha t}, \beta \frac{e^{\alpha t}-1}{\alpha}\right)$, so that the integral curve $I_{\left(a_{0}, b_{0}\right)}$ for $X$ with given initial value $\left(a_{0}, b_{0}\right) \in \mathbb{C} \backslash\{0\} \times \mathbb{C}$ is

$$
\left(a_{0}, b_{0}\right) \cdot \exp t X=\left(a_{0} \exp \alpha t, a_{0} \beta \frac{e^{\alpha t}-1}{\alpha}+b_{0}\right)
$$

so that $I_{\left(a_{0}, b_{0}\right)}$ is a complex line in $\mathbb{C} \backslash\{0\} \times \mathbb{C}$ defined by $\beta a-\alpha b=\beta a_{0}-\alpha b_{0}$. Thus a non-constant, entire integral curve for $X$ is never relatively compact 
in $M$ (this fact also follows from the Liouville theorem). It follows that any smoothly bounded pseudoconvex domain $D \Subset M$ is Stein.

2. Grauert gave an example of a pseudoconvex domain $D$ with smooth boundary which is not Stein. Moreover, $D$ admits no nonconstant holomorphic functions. This domain lies in a complex torus $\mathbf{T}$ of complex dimension 2 (cf., [5] and Example 3 in p. 324 in [15]). Our goal is to describe all pseudoconvex subdomains $D$ of $\mathbf{T}$ with smooth boundary which are not Stein. The key tools we will use are Theorem 5.1 and Remark 5.2

We begin with real 4-dimensional Euclidean space $\mathbb{R}^{4}$ with coordinates $x=\left(x_{1}, x_{2}, x_{3}, x_{4}\right)$. Let

$$
e_{1}=(1,0,0,0), e_{2}=(0,1,0,0), e_{3}=(0,0,1,0), e_{4}=(0,0, \xi, 1) \quad \text { in } \mathbb{R}^{4},
$$

where $\xi$ is an irrational number. Initially we consider the real 4-dimensional torus:

$$
T:=\mathbb{R}^{4} /\left[e_{1}, e_{2}, e_{3}, e_{4}\right]=T_{1} \times T_{2},
$$

where $T_{1}=\mathbb{R}_{x_{1}, x_{2}} /\left[e_{1}^{\prime}, e_{2}^{\prime}\right]$ and $T_{2}=\mathbb{R}_{x_{3}, x_{4}} /\left[e_{3}^{\prime}, e_{4}^{\prime}\right]$, with

$$
\begin{array}{ll}
e_{1}^{\prime}=(1,0), e_{2}^{\prime}=(0,1) & \text { in } \mathbb{R}_{x_{1}} \times \mathbb{R}_{x_{2}} ; \\
e_{3}^{\prime}=(1,0), e_{4}^{\prime}=(\xi, 1) & \text { in } \mathbb{R}_{x_{3}} \times \mathbb{R}_{x_{4}} .
\end{array}
$$

This torus $T$ is a real Lie group with real Lie algebra

$$
\mathfrak{h}=\left\{\sum_{j=1}^{4} c_{j} \frac{\partial}{\partial x_{j}}: c_{j} \in \mathbb{R}\right\}
$$

Following Grauert, we impose the complex structure

$$
z=x_{1}+i x_{3}, \quad w=x_{2}+i x_{4}
$$

on $T$. Then $T$, equipped with this complex structure, becomes a complex torus $\mathbf{T}$ of complex dimension 2. Note that $e_{1}, e_{2}, e_{3}, e_{4}$ correspond to $(1,0),(0,1),(i, 0),(i \xi, i)$ in $\mathbb{C}^{2}$. Grauert showed that

$$
D=D\left(c_{1}, c_{2}\right):=\left\{c_{1}<\Re z<c_{2}\right\} \subset \mathbf{T},
$$

where $0 \leq c_{1}<c_{2}<1$, is a pseudoconvex domain which admits no nonconstant holomorphic functions.

The complex Lie algebra of the complex Lie group $\mathbf{T}$ is

$$
\mathfrak{g}=\left\{\alpha \frac{\partial}{\partial z}+\beta \frac{\partial}{\partial w}: \alpha, \beta \in \mathbb{C}\right\} .
$$




\section{Remark 5.3.}

1. A point $\left(x_{1}, x_{2}, x_{3}, x_{4}\right)$ in $\mathbb{R}^{4}$ (and, more generally, a subset $K \subset \mathbb{R}^{4}$ ) will often be identified with the point $\left(x_{1}, x_{2}, x_{3}, x_{4}\right) /\left[e_{1}, e_{2}, e_{3}, e_{4}\right]$ in $\mathbf{T}$ (and $K /\left[e_{1}, e_{2}, e_{3}, e_{4}\right]$ in $\left.\mathbf{T}\right)$. A similar remark applies to points and subsets in $\mathbb{R}_{x_{1}} \times \mathbb{R}_{x_{2}}$ (or $\mathbb{R}_{x_{3}} \times \mathbb{R}_{x_{4}}$ ) being identified with points and subsets in $T_{1}$ (or $T_{2}$ ). In particular, let $L_{1}$ be a line in $\mathbb{R}_{x_{1}} \times \mathbb{R}_{x_{2}}$ containing the origin. Then $L_{1}$ defines a simple closed curve $l_{1}=L_{1} /\left[e_{1}^{\prime}, e_{2}^{\prime}\right]$ in $T_{1}$ if and only if $L_{1}$ contains a point in $\mathbb{R}_{x_{1}} \times \mathbb{R}_{x_{2}}$ of the form $(m, n)$ where $m, n \in \boldsymbol{Z}$. In this case, there exist unique such $m, n \in \boldsymbol{Z}$ up to a sign with $(m, n)= \pm 1$, and $l_{1}$ may be identified with the segment joining $(0,0)$ and $(m, n)$ in $\mathbb{R}_{x_{1}} \times \mathbb{R}_{x_{2}}$. If we need to consider a directed line $L_{1}$ and an oriented curve $l_{1}$, we fix the direction of $L_{1}$ as that given by the directed line segment from $(0,0)$ to $(m, n)$, and this induces what we will call the positive orientation on $l_{1}$. Moreover, if we fix $m_{1}, n_{1} \in \boldsymbol{Z}$ such that $m_{1} n-m n_{1}=1$, then the parallelogram $\Delta_{1}$ with vertices $(0,0),\left(m_{1}, n_{1}\right),(m, n),\left(m_{1}+m, n_{1}+n\right)$ is a fundamental domain of $T_{1}$, which we call a standard fundamental domain associated to $L_{1}$. (For future convention we choose $m_{1} n-n_{1} m=1$.) The segment joining $(0,0)$ and $\left(m_{1}, n_{1}\right)$ in $\mathbb{R}_{x_{1}} \times \mathbb{R}_{x_{2}}$ defines a simple closed curve in $T_{1}$, which we denote by $l_{1}^{*}$. Note that $\left(m_{1}, n_{1}\right) \in L_{1}+(1 / n, 0)$.

2. Let $L_{2}$ be a line in $\mathbb{R}_{x_{3}} \times \mathbb{R}_{x_{4}}$ containing the origin. Then $L_{2}$ defines a simple closed curve $l_{2}=L_{2} /\left[e_{3}^{\prime}, e_{4}^{\prime}\right]$ in $T_{2}$ if and only if $L_{2}$ contains a point $\left(M^{\prime}, n^{\prime}\right)$ where $M^{\prime}=m^{\prime}+n^{\prime} \xi$ with $m^{\prime}, n^{\prime} \in Z$. Then there exist unique such $m^{\prime} \in \boldsymbol{Z}, n^{\prime} \in \boldsymbol{Z}^{+}$with $\left(m^{\prime}, n^{\prime}\right)= \pm 1$, and $l_{2}$ may be identified with the segment joining $(0,0)$ and $\left(M^{\prime}, n^{\prime}\right)$ in $\mathbb{R}_{x_{3}} \times \mathbb{R}_{x_{4}}$. We fix the direction of $L_{2}$ as that given by the directed line segment from $(0,0)$ to $\left(m^{\prime}+n^{\prime} \xi, n^{\prime}\right)$, and this induces what we will call the positive orientation on $l_{2}$. If we fix $m_{1}^{\prime}, n_{1}^{\prime} \in \boldsymbol{Z}$ such that $m_{1}^{\prime} n^{\prime}-m^{\prime} n_{1}^{\prime}=1$, then the parallelogram $\Delta_{2}$ with vertices $(0,0),\left(M_{1}^{\prime}, n_{1}^{\prime}\right),\left(M_{1}, n_{1}\right),\left(M_{1}^{\prime}+M_{1}, n_{1}^{\prime}+n_{1}\right)\left(\right.$ where $\left.M_{1}^{\prime}=m_{1}^{\prime}+n_{1}^{\prime} \xi\right)$ is a fundamental domain of $T_{2}$, which we call a standard fundamental domain associated to $L_{2}$. The segment joining $(0,0)$ and $\left(M_{1}^{\prime}, n_{1}^{\prime}\right)$ in $\mathbb{R}_{x_{3}} \times \mathbb{R}_{x_{4}}$ defines a simple closed curve in $T_{2}$, which we denote by $l_{2}^{*}$. Note that $\left(M_{1}^{\prime}, n_{1}^{\prime}\right) \in$ $L_{2}+\left(1 / n^{\prime}, 0\right)$.

3. Finally, we mention that if $L_{1}, L_{1}^{\prime}\left(L_{2}, L_{2}^{\prime}\right)$ are distinct parallel lines in $\mathbb{R}_{x_{1}} \times \mathbb{R}_{x_{2}}\left(\mathbb{R}_{x_{3}} \times \mathbb{R}_{x_{4}}\right)$, then for the corresponding curves $l_{1}, l_{1}^{\prime}\left(l_{2}, l_{2}^{\prime}\right)$ in $T_{1}$ $\left(T_{2}\right)$ either $l_{1} \cap l_{1}^{\prime}=\emptyset\left(l_{2} \cap l_{2}^{\prime}=\emptyset\right)$ or $l_{1}=l_{1}^{\prime}\left(l_{2}=l_{2}^{\prime}\right)$.

Let $D \Subset \mathbf{T}$ be a pseudoconvex domain with smooth boundary which is not Stein. We consider the $c$-Robin function $\Lambda(z, w)$ on $D$, where $c \equiv 1$ on T. By Theorem 5.1 there exists $X=\alpha \frac{\partial}{\partial z}+\beta \frac{\partial}{\partial w} \in \mathfrak{g}$ with $(\alpha, \beta) \neq(0,0)$ such that $D(\exp t X)^{-1}=D, t \in \mathbb{C}$. Since the integral curve $\exp t X, t \in \mathbb{C}$ 
for $X$ passing through $\mathbf{0}$ in $\mathbf{T}$ is

$$
(z, w)=(\alpha t, \beta t) \in \mathbb{C}_{z} \times \mathbb{C}_{w}, \quad t \in \mathbb{C},
$$

the above formula means simply that $D+(\alpha t, \beta t)=D, t \in \mathbb{C}$. Since $\operatorname{dim} \mathbf{T}=2$, the Lie subalgebra $\mathfrak{g}_{0}$ associated to $D$ from Theorem 5.1 and the corresponding Lie subgroup $H$ are of the form

$$
\mathfrak{g}_{0}=\{c X \in \mathfrak{g}: c \in \mathbb{C}\}, \quad H=\{(\alpha t, \beta t) \in \mathbf{T}: t \in \mathbb{C}\} .
$$

We consider three cases in (5.13):

$$
\text { (1) } \alpha=0, \quad \text { (2) } \beta=0, \quad \text { (3) } \alpha, \beta \neq 0 \text {. }
$$

In case (1), the subalgebra $\mathfrak{g}_{0}$ is $\left\{c \frac{\partial}{\partial w}: c \in \mathbb{C}\right\}$ and the Lie subgroup

$$
H=\{(0, \beta t): t \in \mathbb{C}\}=\{0\} \times \text { the } w \text {-plane }=\{0\} \times \mathbb{C}_{w} ;
$$

i.e.,

$$
H=\{(0, w) /[(1,0),(0,1),(i, 0),(i \xi, i)]
$$

We shall show that $H$ is not closed in $\mathbf{T}$ and the closure $\bar{H}$ in $\mathbf{T}$ is diffeomorphic to $S^{1} \times T_{2}$. In fact, as a set in $\mathbf{T}=T_{1} \times T_{2}, H$ is equal to

$$
A \times B:=\left(\left(0, \mathbb{R}_{x_{2}}\right) /[(1,0),(0,1)]\right) \times\left(\left(0, \mathbb{R}_{x_{4}}\right) /[(1,0),(\xi, 1)]\right) \subset T_{1} \times T_{2}
$$

Clearly $A$ is closed, and $A=\{0\} \times \mathbb{R}_{x_{2}} /[1]=\{0\} \times S^{1}$. Since $\xi$ is irrational, it is standard that $B$ is not closed in $T_{2}$ and $\bar{B}=T_{2}$. Thus the closure $\bar{H}$ of $H$ in $\mathbf{T}$ can be identified with

$$
\bar{H}=\left(\{0\} \times S^{1}\right) \times T_{2}=\left\{x_{1}=0\right\} /[(1,0),(0,1),(i, 0),(i \xi, i)],
$$

as claimed. We note that $\bar{H}$ is a closed real Lie subgroup of $T$ with real Lie subalgebra $\left\{\sum_{j=2}^{4} c_{j} \frac{\partial}{\partial x_{j}}\right\}$. The quotient space $\mathbf{T} / \bar{H}=S^{1}:=\left\{x_{1} \in \mathbb{R}_{x_{2}} /[1]\right\}$ and the projection $\tilde{\pi}: \mathbf{T} \mapsto \mathbf{T} / \bar{H}$ is $\tilde{\pi}(z, w)=x_{1}$. From Remark [5.2, $D$ is foliated by cosets of $\bar{H}$; thus there exists a subdomain $D_{0}=\left(c_{1}, c_{2}\right) \subset S^{1}$ such that $D=\widetilde{\pi}^{-1}\left(D_{0}\right)$; i.e., $D=\left\{c_{1}<x_{1}<c_{2}\right\} \subset \mathbf{T}$. This is the Grauert example.

In case (2), the subalgebra $\mathfrak{g}_{0}$ is $\left\{c \frac{\partial}{\partial z}: c \in \mathbb{C}\right\}$ and the Lie subgroup

$$
H=\{(\alpha t, 0): t \in \mathbb{C}\}=\text { the } z \text {-plane } \times\{0\}=\mathbb{C}_{z} \times\{0\} ;
$$

i.e.,

$$
H=\left\{(z, 0) /[(1,0),(0,1),(i, 0),(i \xi, i)] \in \mathbf{T}: z \in \mathbb{C}_{z}\right\}
$$


We shall show that $H$ is closed in $\mathbf{T}$ and is holomorphically equivalent to the standard one-dimensional torus $\mathbf{T}_{1}=\mathbb{C} /[1, i]$. In fact, since $z_{1} \sim z_{2}$ modulo $[1, i]$ in $\mathbb{C}_{z}$ if and only if $\left(z_{1}, 0\right) \sim\left(z_{2}, 0\right)$ modulo $[(1,0),(0,1),(i, 0),(i \xi, i)]$ in $\mathbb{C}^{2}$, it follows that

$$
H=\mathbb{C}_{z} /[1, i] \times\{0\}
$$

so that $H=\mathbf{T}_{1} \times\{0\}$, as claimed. Moreover, we have $\left(z_{1}, w_{1}\right)+H=\left(z_{2}, w_{2}\right)+$ $H$ in $\mathbf{T}$ if and ony if $w_{1} \sim w_{2}$ modulo [1,i]. The "only if" direction is clear. To prove the reverse direction, fix $\left(z_{1}, w_{1}\right),\left(z_{2}, w_{2}\right) \in \mathbb{C}^{2}$ with $w_{1} \sim w_{2}$ modulo $[1, i]$, i.e., $w_{2}-w_{1}=p+i q$ for some $p, q \in \boldsymbol{Z}$. Since $(z, p+q i)-(z-q \xi i, 0)=$ $p(0,1)+q(i \xi, i)=(0,0)$ in $\mathbf{T}$ for any $z \in \mathbb{C}$, it follows that

$$
\begin{aligned}
\left(z_{2}, w_{2}\right) & =\left(z_{1}, w_{1}\right)+\left(z_{2}-z_{1}, p+q i\right) \\
& =\left(z_{1}, w_{1}\right)+\left(z_{2}-z_{1}-q \xi i, 0\right) \in\left(z_{1}, w_{1}\right)+H \quad \text { in } \mathbf{T},
\end{aligned}
$$

as claimed. In particular, $(z, w)+H=(0, w)+H$ in $\mathbf{T}$ for any $z \in \mathbb{C}$, and $(0, w)+H=\left(0, w^{\prime}\right)+H$ in $\mathbf{T}$ if and only if $w \sim w^{\prime}$ modulo $[1, i]$. Hence $\mathbf{T} / H=\{0\} \times \mathbb{C}_{w} /[1, i]=\{0\} \times \mathbf{T}_{1}$, so that $\mathbf{T}=\mathbf{T}_{1} \times \mathbf{T}_{1}$. By II in Theorem 5.1, using the projection $\pi: \mathbf{T} \mapsto \mathbf{T} / H=\mathbf{T}_{1}$, we see that there exists a domain $D_{0} \subset \mathbf{T}_{1}$ with smooth boundary such that $D=\pi^{-1}\left(D_{0}\right)$.

We consider case (3). Write $\beta / \alpha=a+i b$. Then the integral curve $\{\exp t X, t \in \mathbb{C}\}$ starting at $(0,0)$ can be written as

$$
S: \quad w=(a+i b) z \quad \text { in } \mathbb{C}_{z} \times \mathbb{C}_{w}
$$

so that $S /[(1,0),(0,1),(i, 0),(i \xi, \xi)]$ is the Lie subgroup $H$ of $\mathbf{T}$. For future use we note that

$$
S: x_{2}+i x_{4}=(a+i b)\left(x_{1}+i x_{3}\right) \quad \text { in } \mathbb{R}^{4}
$$

defines a real two-dimensional plane passing through the origin in $\mathbb{R}^{4}$; moreover we may identify $H$ with $S /\left[e_{1}, e_{2}, e_{3}, e_{4}\right]$.

We consider two subcases:

$$
\text { (i) } b=0 ; \quad \text { (ii) } b \neq 0 \text {. }
$$

Note that in case (i) $S=\left\{x_{2}=a x_{1}, x_{4}=a x_{3}\right\}$ is the product of two (not necessarily closed) curves $H_{1}=\left\{x_{2}=a x_{1}\right\} \subset T_{1}$ and $H_{2}=\left\{x_{4}=a x_{3}\right\} \subset T_{2}$ in $\mathbf{T}$.

We also make the distinction between the two options:

(i') $a$ is rational; $a=q / p, p, q$ relatively prime; (ii') $a$ is irrational. 
In case (i'), $H_{1}$ defines a closed curve $\gamma_{1}$ in $T_{1}$ determined by the segment $[(0,0),(p, q)]$ in $\mathbb{R}^{2}$, while $H_{2}$ is dense in $T_{2}$. Hence $\bar{H}=\gamma_{1} \times T_{2}$ is a closed real Lie subgroup in $\mathbf{T}$ with corresponding real Lie subalgebra given by

$$
\mathfrak{h}_{0}=\left\{c_{1}\left(\frac{\partial}{\partial x_{1}}+a \frac{\partial}{\partial x_{2}}\right)+c_{2} \frac{\partial}{\partial x_{3}}+c_{3} \frac{\partial}{\partial x_{4}}: c_{1}, c_{2}, c_{3} \in \mathbb{R}\right\} .
$$

Using the projection $\widetilde{\pi}: \mathbf{T} \rightarrow \mathbf{T} / \bar{H}$ yields that $\mathbf{T} / \bar{H}$ is diffeomorphic to $S^{1}=\mathbb{R} /[1 / q]$. From Remark 5.2 there exist $0 \leq a_{1}<a_{2}<1 / q$ such that $D=\pi^{-1}\left(a_{1}, a_{2}\right)=\cup_{a_{1}<s<a_{2}}\left((s, 0 ; 0,0)+\gamma_{1} \times T_{2}\right)$. Thus, in case (i'),

$$
D \approx\left(a_{1}, a_{2}\right) \times \gamma_{1} \times T_{2}
$$

as a two-dimensional complex manifold.

Case (ii') will be divided further into two subcases depending on whether $\frac{1}{a}-\xi$ is rational (i.e., $\frac{1}{a}-\xi=p / q$ ) or irrational. The former case is similar to case (i'): since $q=a(q \xi+p)$, we have $(q \xi+p, q) \in H_{2}$. This point is equivalent to $(0,0) \in T_{2}$, so that $H_{2}$ defines a simple, closed curve $\gamma_{2}$ in $T_{2}$ (see Remark 5.3). On the other hand, since $a$ is irrational, $H_{1}$ is dense in $T_{1}$. Hence $\bar{H}=T_{1} \times \gamma_{2}$, which is a real Lie subgroup of $\mathbf{T}$. The quotient space $\mathbf{T} / \bar{H}=\mathbb{R} /[1 / q]=: S^{1}$. Again from Remark 5.2 there exist $0 \leq a_{1}<a_{2}<1 / q$ such that

$$
D=\bigcup_{a_{1}<s<a_{2}}\left(T_{1} \times \gamma_{2}+(0,0 ; s, 0)\right) .
$$

In case $\frac{1}{a}-\xi$ is irrational, $H_{1}$ and $H_{2}$ are dense in $T_{1}$ and $T_{2}$. But $H_{1} \times H_{2} \Subset D$, so that $T_{1} \times T_{2} \Subset D$, which yields a contradiction since $D \neq \mathbf{T}$. In other words, the case $\frac{1}{a}-\xi$ irrational cannot occur.

We turn to case (ii) in our previous dichotomy (5.15): $b \neq 0$. Here $a$ may be either rational or irrational. From (5.14) $S \subset \mathbb{R}^{4}$ may be written as

$$
\left\{\begin{array}{l}
x_{2}=a x_{1}-b x_{3}, \\
x_{4}=b x_{1}+a x_{3},
\end{array} \quad\left(x_{1}, x_{3}\right) \in \mathbb{R}_{x_{1}} \times \mathbb{R}_{x_{3}}\right.
$$

or, equivalently,

$$
\left\{\begin{array}{l}
x_{3}=A x_{1}+B x_{2}, \\
x_{4}=C x_{1}-A x_{2},
\end{array} \quad\left(x_{3}, x_{4}\right) \in \mathbb{R}_{x_{3}} \times \mathbb{R}_{x_{4}},\right.
$$

where

$$
A=\frac{a}{b}, \quad B=-\frac{1}{b}, \quad C=\frac{a^{2}+b^{2}}{b} \neq 0 .
$$

Note that $A, B, C$ are related via the Jacobian:

$$
\frac{\partial\left(x_{3}, x_{4}\right)}{\partial\left(x_{1}, x_{2}\right)}=-A^{2}-B C=1 .
$$


We will show the following:

1. (i) There exists a unique set of six integers $m, n, m^{\prime} \in \boldsymbol{Z} ; n^{\prime}, p, q \in \boldsymbol{Z}^{+}$ where $(m, n),\left(m^{\prime}, n^{\prime}\right)= \pm 1,(p, q)=1$, such that $a, b$ can be written in the following form:

$$
a=\frac{p_{1} p_{2}+q_{1} q_{2}}{p_{1}^{2}+q_{1}^{2}}, \quad b=\frac{p_{2} q_{1}-p_{1} q_{2}}{p_{1}^{2}+q_{1}^{2}}
$$

where

$$
M^{\prime}:=m^{\prime}+n^{\prime} \xi ; \quad p_{1}:=M^{\prime} p, \quad p_{2}:=n^{\prime} p, \quad q_{1}:=m q, \quad q_{2}:=n q .
$$

1. (ii) The integral curve $S$ in (5.14) contains the following two points:

$$
\left(q(m, n), p\left(M^{\prime}, n^{\prime}\right)\right), \quad\left(p(1 / n, 0), q\left(1 / n^{\prime}, 0\right)+\eta\left(M^{\prime}, n^{\prime}\right)\right),
$$

where $\eta=\frac{p}{n n^{\prime}} \frac{p_{2}^{2}+q_{2}^{2}}{p_{2} q_{1}-p_{1} q_{2}}$. Note that $\eta$ is irrational.

2. (i) The closure $\bar{H}$ of $H$ in $\mathbf{T}$ is a closed real Lie subgroup of $\mathbf{T}$ whose corresponding real Lie subalgebra $\mathfrak{h}_{0}$ of $\mathfrak{h}$ is generated by

$$
\begin{aligned}
& \left\{q_{1} \frac{\partial}{\partial x_{1}}+q_{2} \frac{\partial}{\partial x_{2}}, p_{1} \frac{\partial}{\partial x_{3}}+p_{2} \frac{\partial}{\partial x_{4}}\right. \\
& \left.\quad\left(p_{2} q_{1}-p_{1} q_{2}\right) \frac{\partial}{\partial x_{1}}+\left(p_{1} p_{2}+q_{1} q_{2}\right) \frac{\partial}{\partial x_{3}}+\left(p_{2}^{2}+q_{2}^{2}\right) \frac{\partial}{\partial x_{4}}\right\} .
\end{aligned}
$$

We proceed to give a more precise description of $\bar{H}$. Assuming 1., let

$$
\begin{array}{lll}
L_{1}:\left\{\left(x_{1}, x_{2}\right): m x_{2}=n x_{1}\right\}=\{t(m, n): t \in \mathbb{R}\} & \text { in } \mathbb{R}_{x_{1}} \times \mathbb{R}_{x_{2}} \\
L_{2}:\left\{\left(x_{3}, x_{4}\right): M^{\prime} x_{4}=n^{\prime} x_{3}\right\}=\left\{t\left(M^{\prime}, n^{\prime}\right): t \in \mathbb{R}\right\} & \text { in } \mathbb{R}_{x_{3}} \times \mathbb{R}_{x_{4}} .
\end{array}
$$

Since $m, n \in \boldsymbol{Z}, L_{1}$ defines a simple closed curve $l_{1}$ with positive orientation in the real torus $T_{1}$ (see 1. in Remark 5.3), and from (5.18), specifically, the relation $M^{\prime}=m^{\prime}+n^{\prime} \xi, L_{2}$ defines a simple closed curve $l_{2}$ with positive orientation in the real torus $T_{2}$ (see 2. in Remark 5.3). Given $0 \leq s \leq 1$, define

$$
\begin{array}{lll}
\mathcal{L}_{1}(s):=L_{1}+p s(1 / n, 0)=\{t(m, n)+p s(1 / n, 0): t \in \mathbb{R}\} & \text { in } \mathbb{R}_{x_{1}} \times \mathbb{R}_{x_{2}} \\
\mathcal{L}_{2}(s):=L_{2}+q s\left(1 / n^{\prime}, 0\right)=\left\{t\left(M^{\prime}, n^{\prime}\right)+q s\left(1 / n^{\prime}, 0\right): t \in \mathbb{R}\right\} & \text { in } \mathbb{R}_{x_{3}} \times \mathbb{R}_{x_{4}}
\end{array}
$$

Then $\mathcal{L}_{1}(s)$ and $\mathcal{L}_{2}(s)$ also define simple closed curves $l_{1}(s)$ and $l_{2}(s)$ in $T_{1}$ and $T_{2} ; l_{1}(s)$ is a curve in $T_{1}$, which, in $\mathbb{R}_{x_{1}} \times \mathbb{R}_{x_{2}}$, is parallel to $l_{1}$ translated 
by the vector $p s\left(\frac{1}{n}, 0\right)$. Similarly, $l_{2}(s)$ is parallel to $l_{2}$ in $\mathbb{R}_{x_{3}} \times \mathbb{R}_{x_{4}}$ translated by the vector $q s\left(\frac{1}{n^{\prime}}, 0\right)$. We have $l_{i}=l_{i}(0)=l_{i}(1)$ for $i=1,2$ and

$$
\left(l_{1}\left(s^{\prime}\right) \times l_{2}\left(s^{\prime}\right)\right) \cap\left(l_{1}\left(s^{\prime \prime}\right) \times l_{2}\left(s^{\prime \prime}\right)\right)=\emptyset \text { if } s^{\prime} \neq s^{\prime \prime} .
$$

Note that we may have, e.g., $l_{1}\left(s^{\prime}\right)=l_{1}\left(s^{\prime \prime}\right)$ if $s^{\prime} \neq s^{\prime \prime}$ but then $l_{2}\left(s^{\prime}\right) \neq l_{2}\left(s^{\prime \prime}\right)$ from $(p, q)=1$ so that (5.20) holds (cf., 3. in Remark 5.3).

2. (ii) The set

$$
\Sigma:=\bigcup_{0 \leq s \leq 1} l_{1}(s) \times l_{2}(s)
$$

is a real, 3-dimensional compact submanifold of $\mathbf{T}$, and $\bar{H}=\Sigma$. Given $0 \leq t \leq 1$, if we define

$$
\Sigma(t):=(t, 0 ; 0,0)+\bar{H}=(t, 0 ; 0,0)+\Sigma,
$$

a coset of $\bar{H}$, then $\Sigma(0)=\Sigma(1)=\Sigma=\bar{H}$ and otherwise $\Sigma\left(t^{\prime}\right) \cap \Sigma\left(t^{\prime \prime}\right)=\emptyset$ in $\mathbf{T}$ if $t^{\prime} \neq t^{\prime \prime}$.

3. We have

$$
\mathbf{T}=\bigcup_{0 \leq t \leq 1} \Sigma(t) .
$$

The quotient space $\mathbf{T} / \bar{H}=\mathbb{R} /[1]=S^{1}$ and

$$
D=\bigcup_{t_{1}<t<t_{2}} \Sigma(t)
$$

where $0 \leq t_{1}<t_{2}<1$.

We will also prove a converse statement:

4. Given integers $m, n, m^{\prime} \in \boldsymbol{Z} ; n^{\prime}, p, q \in \boldsymbol{Z}^{+}$with $(m, n),\left(m^{\prime}, n^{\prime}\right)=$ $\pm 1,(p, q)=1$, we can find $a, b \in \mathbb{R}$ satisfying (5.17) and (5.18) to construct $a$ pseudoconvex domain $D \subset \mathbf{T}$ with smooth boundary which is not Stein. This domain has the property that $D(\tau \exp t X)^{-1}=D$ for all $t \in \mathbb{C}$ and for all $\tau \in$ $D$ where $X$ is a nonzero holomorphic vector field with the property that the Lie subgroup $H$ of $\mathbf{T}$ corresponding to the Lie subalgebra $\mathfrak{g}_{0}=\{c X \in \mathfrak{g}: c \in \mathbb{C}\}$ is equal to $\{w=(a+b i) z\} /\left[e_{1}, e_{2}, e_{3}, e_{4}\right]$. Moreover, every holomorphic function on $D$ is constant.

We proceed with the proofs of items 1 . through 4. Following (5.16) we write

$$
\begin{aligned}
F:\left(x_{1}, x_{2}\right) & \mapsto\left(x_{3}, x_{4}\right)=\left(A x_{1}+B x_{2}, C x_{1}-A x_{2}\right), \\
F^{-1}:\left(x_{3}, x_{4}\right) & \mapsto\left(x_{1}, x_{2}\right)=\left(-A x_{3}-B x_{4},-C x_{3}+A x_{4}\right)
\end{aligned}
$$


so that $F, F^{-1}$ are linear mappings and

$$
H=\left\{\left(x_{1}, x_{2}, F\left(x_{1}, x_{2}\right)\right) \in \mathbb{R}^{4}:\left(x_{1}, x_{2}\right) \in \mathbb{R}_{x_{1}} \times \mathbb{R}_{x_{2}}\right\} /\left[e_{1}, e_{2}, e_{3}, e_{4}\right] \quad \text { in } \mathbf{T} .
$$

By Sard's theorem there exists a point $x^{0} \in D$ such that

$$
\nabla \Lambda\left(x^{0}\right)=\left(\frac{\partial \Lambda}{\partial x_{1}}, \ldots, \frac{\partial \Lambda}{\partial x_{4}}\right)\left(x^{0}\right) \neq \mathbf{0}
$$

The $c$-Robin function $\Lambda(x)$ is invariant under parallel translation in $\mathbb{R}^{4}$ since the operator $\Delta-c=\Delta-1$ associated to the Euclidean metric $\sum_{i=1}^{4} d x_{i}^{2}$ is invariant under parallel translation. Thus if we write $D_{1}:=D-x^{0}$ in $\mathbf{T}$ and $\Lambda_{1}(x)$ is the $c$-Robin function for $D_{1}$, then $\Lambda_{1}(x)=\Lambda\left(x+x^{0}\right)$. Therefore we may assume that $D$ contains the origin $\mathbf{0}$ and $\nabla \Lambda(\mathbf{0}) \neq \mathbf{0}$. Thus $H \Subset D$ and $\Lambda(x) \equiv \Lambda(\mathbf{0})$ on $H$.

For $m, n \in \boldsymbol{Z}$, we set

$$
P(m, n):=(m, n, F(m, n))=(m, n, A m+B n, C m-A n) \in H .
$$

Note that such a point is equal to $(0,0, F(m, n))=(0,0, A m+B n, C m-A n)$ in $\mathbf{T}$. We set $\mathcal{P}:=\left\{F(m, n) \in \mathbb{R}_{x_{3}} \times \mathbb{R}_{x_{4}}: m, n \in \boldsymbol{Z}\right\}$ and

$$
\widehat{\mathcal{P}}=\left\{F(m, n)+(K, l) \in \mathbb{R}_{x_{3}} \times \mathbb{R}_{x_{4}}: m, n, k, l \in \boldsymbol{Z}\right\}
$$

where $K=k+l \xi$. Then $\mathcal{P} /\left[e_{3}^{\prime}, e_{4}^{\prime}\right]=\widehat{\mathcal{P}} /\left[e_{3}^{\prime}, e_{4}^{\prime}\right]$; i.e., $\mathcal{P}$ and $\widehat{\mathcal{P}}$ define the same set in $T_{2}$. We show:

The closure $C l[\widehat{\mathcal{P}}]$ of $\widehat{\mathcal{P}}$ in $\mathbb{R}_{x_{3}} \times \mathbb{R}_{x_{4}}$ consists of an infinite number of parallel lines the same distance apart, one of which passes through the origin $(0,0)$.

Note that, by definition, $\widehat{\mathcal{P}}$ is an additive subgroup in $\mathbb{R}_{x_{3}} \times \mathbb{R}_{x_{4}}$. Moreover, $\widehat{\mathcal{P}}$ cannot lie on a single line so that $C l[\widehat{\mathcal{P}}]$ is not a single line containing the origin. To verify the italicized statement we must first rule out two other possibilities:

i. $\widehat{\mathcal{P}}$ is an isolated set in $\mathbb{R}_{x_{3}} \times \mathbb{R}_{x_{4}}$;

ii. $C l[\widehat{\mathcal{P}}]=\mathbb{R}_{x_{3}} \times \mathbb{R}_{x_{4}}$.

To prove that i. does not occur, let $\Delta$ be a fundamental parallelogram of $T_{2}$ with vertices $(0,0),(1,0),(\xi, 1)$, and $(1+\xi, 1)$. Then each point $P(m, n)$ in $\mathcal{P}$ is equivalent to some point $P^{\prime}(m, n)$ in $\Delta$. Since $\Delta$ is bounded, it is enough 
to show that $\left\{P^{\prime}(m, n)\right\}_{m, n \in \boldsymbol{Z}}$ consists of infinitely many distinct points in $\Delta$. We first verify that for any three pairs $\left(m_{i}, n_{i}\right) \in \boldsymbol{Z} \times \boldsymbol{Z}, i=0,1,2$,

$$
P^{\prime}\left(m_{0}, n_{0}\right)=P^{\prime}\left(m_{1}, n_{1}\right)=P^{\prime}\left(m_{2}, n_{2}\right) \text { implies } \frac{m_{1}-m_{0}}{n_{1}-n_{0}}=\frac{m_{2}-m_{0}}{n_{2}-n_{0}} .
$$

For the sake of obtaining a contradiction, suppose $\frac{m_{1}-m_{0}}{n_{1}-n_{0}} \neq \frac{m_{2}-m_{0}}{n_{2}-n_{0}}$. The condition $P^{\prime}\left(m_{0}, n_{0}\right)=P^{\prime}\left(m_{1}, n_{1}\right)=P^{\prime}\left(m_{2}, n_{2}\right)$ implies that there exist $\left(p_{i}, q_{i}\right) \in \boldsymbol{Z} \times \boldsymbol{Z}, i=0,1,2$ such that the three points

$$
\left\{\left(A m_{i}+B n_{i}-\left(p_{i}+q_{i} \xi\right), C m_{i}-A n_{i}-q_{i}\right)\right\}_{i=0,1,2}
$$

are the same in $\Delta$. This means that

$$
\begin{gathered}
A\left(m_{1}-m_{0}\right)+B\left(n_{1}-n_{0}\right)=\left(p_{1}-p_{0}\right)+\left(q_{1}-q_{0}\right) \xi, \\
A\left(m_{2}-m_{0}\right)+B\left(n_{2}-n_{0}\right)=\left(p_{2}-p_{0}\right)+\left(q_{2}-q_{0}\right) \xi, \\
C\left(m_{1}-m_{0}\right)-A\left(n_{1}-n_{0}\right)=q_{1}-q_{0}, \\
C\left(m_{2}-m_{0}\right)-A\left(n_{2}-n_{0}\right)=q_{2}-q_{0} .
\end{gathered}
$$

From the last two equations it follows that $C$ and $A$ are rational. Using the relation $A^{2}+B C+1=0, B$ must be rational as well. Since $\xi$ is irrational, we have $q_{1}-q_{0}=q_{2}-q_{0}=0$; hence $A=C=0$, yielding a contradiction. This proves (5.25). Now suppose $\left\{P^{\prime}(m, n)\right\}_{m, n \in \boldsymbol{Z}}$ is a finite set in $\Delta$. Then $\left\{\frac{m_{1}-m_{0}}{n_{1}-n_{0}}\right\}_{m_{0}, m_{1}, n_{0}, n_{1} \in \boldsymbol{Z}}$ would have to be a finite set of rational numbers, which it is not.

We next rule out case ii. Assume, for the sake of obtaining a contradiction, that $C l[\widehat{\mathcal{P}}]=\mathbb{R}_{x_{3}} \times \mathbb{R}_{x_{4}}$. Since $\Lambda(x) \equiv \Lambda(\mathbf{0})$ on $H$ and since $(0,0 ; \mathcal{P}) \subset H$, from continuity it follows that $\Lambda(x) \equiv \Lambda(\mathbf{0})$ on $(0,0 ; C l[\widehat{\mathcal{P}}])$. Hence $\Lambda\left(0,0 ; x_{3}, x_{4}\right) \equiv \Lambda(\mathbf{0})$ on $T_{2}$. In particular, $\frac{\partial \Lambda}{\partial x_{3}}=\frac{\partial \Lambda}{\partial x_{4}}=0$ at $\mathbf{0}$. Moreover, since $\Lambda \equiv \Lambda(\mathbf{0})$ on $H=\{w=(a+b i) z\} /\left[e_{1}, e_{2}, e_{3}, e_{4}\right]$, i.e., $\Lambda(z,(a+b i) z) \equiv \Lambda(\mathbf{0})$ in $\mathbb{C}_{z}$ where $z=x_{1}+i x_{3}$, we have

$$
\frac{\partial \Lambda}{\partial z}+(a+b i) \frac{\partial \Lambda}{\partial w} \equiv 0
$$

on $H$. Equivalently,

$$
\frac{\partial \Lambda}{\partial x_{1}}-i \frac{\partial \Lambda}{\partial x_{3}}+(a+b i)\left(\frac{\partial \Lambda}{\partial x_{2}}-i \frac{\partial \Lambda}{\partial x_{4}}\right) \equiv 0 \quad \text { at } \quad \mathbf{0} .
$$

Since $\frac{\partial \Lambda}{\partial x_{3}}=\frac{\partial \Lambda}{\partial x_{4}}=0$ at $\mathbf{0}$ and $b \neq 0$ it follows that $\frac{\partial \Lambda}{\partial x_{1}}=\frac{\partial \Lambda}{\partial x_{2}}=0$ at $\mathbf{0}$. This contradicts the hypothesis that $\nabla \Lambda(\mathbf{0}) \neq \mathbf{0}$.

We conclude that $C l[\widehat{\mathcal{P}}]=\cup_{\nu=-\infty}^{\infty} L_{2}^{\nu}$, where $L_{2}:=L_{2}^{0}$ passes through the origin, and $L_{2}^{\nu}=L_{2}+\nu(d, 0)$ for some $d>0$; i.e., $L_{2}^{\nu}$ is parallel to $L_{2}$ 
translated by $\nu(d, 0)$ along the $x_{3}$-axis. By a similar calculation as in (5.26) one can check that the line $L_{2}$ cannot be either the $x_{3}$-axis or the $x_{4}$-axis.

We claim that the line $L_{2}$ defines a simple closed curve $l_{2}:=L_{2} /[(1,0),(\xi, 1)]$ in $T_{2}$. Indeed, if not, $L_{2}$ defines a non-closed curve which must be dense in $T_{2}$. Since $\Lambda\left(0,0 ; L_{2}\right) \equiv \Lambda(\mathbf{0})$, we have $\Lambda\left(0,0 ; T_{2}\right)=\Lambda(\mathbf{0})$. As above, this contradicts $\nabla \Lambda(\mathbf{0}) \neq \mathbf{0}$.

There thus exists a unique $m^{\prime} \in \boldsymbol{Z}, n^{\prime} \in \boldsymbol{Z}^{+}$with $\left(m^{\prime}, n^{\prime}\right)= \pm 1$ such that $L_{2}$ passes through

$$
m^{\prime}(1,0)+n^{\prime}(\xi, 1)=\left(m^{\prime}+n^{\prime} \xi, n^{\prime}\right)=:\left(M^{\prime}, n^{\prime}\right)
$$

which is equal to $(0,0)$ as a point in $T_{2}$. Following 2. in Remark 5.3, we give $l_{2}$ a positive orientation determined from that of $L_{2}$ directed from $(0,0)$ to $\left(M^{\prime}, n^{\prime}\right)$. Considering the standard fundamental domain $\left(\Delta^{\prime \prime}\right)$ of $T_{2}$ associated to $L_{2}$ defined by

$$
\left\{(0,0),\left(M_{1}^{\prime}, n_{1}^{\prime}\right),\left(M^{\prime}, n^{\prime}\right),\left(M_{1}^{\prime}+M^{\prime}, n_{1}^{\prime}+n^{\prime}\right)\right\}
$$

with $m_{1}^{\prime} n_{1}-m_{1} n_{1}^{\prime}=1$, we see that the distance $d$ between successive curves $L_{2}^{\nu}$, e.g., between $L_{2}$ and $L_{2}(1)$, must be of the form $d=\frac{l}{k n^{\prime}}$ where $k, l \in \boldsymbol{Z}$ with $(k, l)= \pm 1$. For otherwise $\cup_{\nu=-\infty}^{\infty} L_{2}^{\nu}$ is dense in $\mathbb{R}_{x_{3}} \times \mathbb{R}_{x_{4}}$. Since $(0,0 ; \widehat{\mathcal{P}}) \subset H$ and hence $(0,0 ; C l[\widehat{\mathcal{P}}])=\left(0,0 ; \cup_{\nu=-\infty}^{\infty} L_{2}^{\nu}\right) \subset \bar{H}$, we again get a contradiction to $\nabla \Lambda(\mathbf{0}) \neq \mathbf{0}$. We will determine $k$ and $l$ explicitly in Remark 5.4 .

Define $L_{1}:=F^{-1}\left(L_{2}\right) \subset \mathbb{R}_{x_{1}} \times \mathbb{R}_{x_{2}}$. We show:

1. $L_{1} \times L_{2} \subset \bar{H}$ and $H+L_{1} \times L_{2} \subset \bar{H}$;

2. $L_{1}$ defines a simple closed curve $l_{1}$ in $T_{1}$;

3. $\Lambda(x) \equiv \Lambda(\mathbf{0})$ on $L_{1} \times L_{2}$.

We first prove the following inclusion:

$$
\text { (*) } H+\left(0,0 ; L_{2}\right) \subset \bar{H} .
$$

To prove this, fix $\left(x_{1}, x_{2} ; F\left(x_{1}, x_{2}\right)\right) \in H$ and $F(m, n)+[k(1,0)+l(\xi, 1)] \in \widehat{\mathcal{P}}$. Then, as points in $\mathbf{T}$,

$$
\begin{aligned}
& \left(x_{1}, x_{2} ; F\left(x_{1}, x_{2}\right)+F(m, n)+[k(1,0)+l(\xi, 1)]\right) \\
& =\left(x_{1}+m, x_{2}+n ; F\left(x_{1}+m, x_{2}+n\right)\right) .
\end{aligned}
$$

By definition, this point is in $H$. We conclude that for all $\left(x_{1}, x_{2} ; F\left(x_{1}, x_{2}\right)\right) \in$ $H$ we have $\left(x_{1}, x_{2} ; F\left(x_{1}, x_{2}\right)+\widehat{\mathcal{P}}\right) \subset H$. Since $L_{2} \subset C l[\widehat{\mathcal{P}}]$, it follows that $(*)$ holds. 
We verify 1 . and 3.: Fix $p:=\left(x_{1}^{0}, x_{2}^{0} ; x_{3}^{\prime}, x_{4}^{\prime}\right) \in L_{1} \times L_{2}$. Then there exists $\left(x_{3}^{0}, x_{4}^{0}\right) \in L_{2}$ with $F\left(x_{1}^{0}, x_{2}^{0}\right)=\left(x_{3}^{0}, x_{4}^{0}\right)$ so that

$$
p=\left(x_{1}^{0}, x_{2}^{0} ; x_{3}^{\prime}, x_{4}^{\prime}\right)=\left(x_{1}^{0}, x_{2}^{0} ; F\left(x_{1}^{0}, x_{2}^{0}\right)\right)+\left(0,0 ; x_{3}^{\prime}-x_{3}^{0}, x_{4}^{\prime}-x_{4}^{0}\right) .
$$

Since $\left(x_{3}^{\prime}-x_{3}^{0}, x_{4}^{\prime}-x_{4}^{0}\right) \in L_{2}-L_{2}=L_{2}$, we have $p \in H+\left(0,0 ; L_{2}\right)$. Then (*) implies $p \in \bar{H}$, so that $L_{1} \times L_{2} \subset \bar{H}$. Since $\bar{H}$ is an additive group, we conclude that $H+L_{1} \times L_{2} \subset \bar{H}$ and 1. is proved. Since $\Lambda(x) \equiv \Lambda(\mathbf{0})$ on $\bar{H}$, 1. implies 3 .

We prove 2 . by contradiction. If 2 . is not true, then $L_{1}$ is dense in $\mathbb{R}_{x_{1}} \times \mathbb{R}_{x_{2}}$, or, equivalently, $L_{1} /\left[e_{1}^{\prime}, e_{2}^{\prime}\right]$ is dense in $T_{1}$. It follows from 1 . that $T_{1} \times L_{2} \subset \bar{H}$, and hence $\Lambda(x) \equiv \Lambda(\mathbf{0})$ on $T_{1} \times L_{2}$. In particular, this holds on $\left(T_{1} ; 0,0\right)$. Using an argument similar to that used in the proof that $C l[\widehat{\mathcal{P}}] \neq \mathbb{R}_{x_{3}} \times \mathbb{R}_{x_{4}}$, this together with $\Lambda\left(x_{1}, x_{2} ; F\left(x_{1}, x_{2}\right)\right) \equiv \Lambda(\mathbf{0})$ for $\left(x_{1}, x_{2}\right) \in \mathbb{R}_{x_{1}} \times \mathbb{R}_{x_{2}}$ implies $\nabla \Lambda(\mathbf{0})=\mathbf{0}$, a contradiction.

Before proceeding, we make a remark about the orientation of the curves $l_{1}$ and $l_{2}$. Recall we fix the direction of the line $L_{2}$ as that given by the directed line segment from $(0,0)$ to $\left(M^{\prime}, n^{\prime}\right)$ in $\mathbb{R}_{x_{3}} \times \mathbb{R}_{x_{4}}$; this determines the positive orientation of $l_{2}$ in $T_{2}$. Similary, we fix the direction of $L_{1}$ as that given by the directed line segment from $(0,0)$ to $F^{-1}\left(M^{\prime}, n^{\prime}\right)$ in $\mathbb{R}_{x_{1}} \times \mathbb{R}_{x_{2}}$, which induces the positive orientation of $l_{1}$ in $T_{1}$. We say that $F$ maps the positively oriented curve $l_{1}$ to the positively oriented curve $l_{2}$. It follows from 1. in Remark 5.3 and assertion 2. that there exist unique $m, n \in \boldsymbol{Z}$ with the property that $(m, n)= \pm 1$ such that the closed curve $l_{1}$ has a positive orientation associated with that of the directed line segment from $(0,0)$ to $(m, n)$.

We replace $\mathcal{P}=\left\{F(m, n) \in \mathbb{R}_{x_{3}} \times \mathbb{R}_{x_{4}}: m, n \in \boldsymbol{Z}\right\}$ in $T_{1}$ by $\mathcal{Q}:=$ $\left\{F^{-1}\left(M^{\prime}, n^{\prime}\right) \in \mathbb{R}_{x_{1}} \times \mathbb{R}_{x_{2}}: m^{\prime}, n^{\prime} \in \boldsymbol{Z}\right\}$ in $T_{2}$, where $M^{\prime}=m^{\prime}+n^{\prime} \xi$. Similarly, we replace

$$
\widehat{\mathcal{P}}=\left\{F(m, n)+(K, l) \in \mathbb{R}_{x_{3}} \times \mathbb{R}_{x_{4}}: m, n, k, l \in \boldsymbol{Z}\right\}
$$

by

$$
\widehat{\mathcal{Q}}=\left\{F^{-1}\left(M^{\prime}, n^{\prime}\right)+\left(k^{\prime}, l^{\prime}\right) \in \mathbb{R}_{x_{1}} \times \mathbb{R}_{x_{2}}: m^{\prime}, n^{\prime}, k^{\prime}, l^{\prime} \in \boldsymbol{Z}\right\} .
$$

As we found $L_{2} \subset C l[\widehat{\mathcal{P}}]$, there exists a line $\widetilde{L}_{1} \subset C l[\widehat{\mathcal{Q}}]$ in $\mathbb{R}_{x_{1}} \times \mathbb{R}_{x_{2}}$ passing through the origin $(0,0)$. This line is neither the $x_{1}$-axis nor the $x_{2}$-axis. We claim that

$$
\widetilde{L}_{1}=L_{1} \quad \text { in } \mathbb{R}_{x_{1}} \times \mathbb{R}_{x_{2}}
$$

To verify this we set $\widetilde{L}_{2}=F\left(\widetilde{L}_{1}\right)$ and we assume, for the sake of obtaining a contradiction, that $\widetilde{L}_{2} \neq L_{2}$. From 3 . applied to $\widetilde{L}_{1}$ and $\widetilde{L}_{2}$, we have 
$\Lambda(x) \equiv \Lambda(\mathbf{0})$ on $\widetilde{L}_{1} \times \widetilde{L}_{2}$. It follows that $\Lambda(x) \equiv \Lambda(\mathbf{0})$ on the following four distinct lines passing through the origin in $\mathbb{R}^{4}$ :

$$
\left(L_{1} ; 0,0\right), \quad\left(0,0 ; L_{2}\right), \quad\left(\widetilde{L}_{1} ; 0,0\right), \quad\left(0,0 ; \widetilde{L}_{2}\right) .
$$

We conclude that $\nabla \Lambda(\mathbf{0})=\mathbf{0}$, a contradiction.

We define the lines $L_{2}(\nu):=L_{2}+\nu\left(\frac{1}{n^{\prime}}, 0\right), \nu=0, \pm 1, \pm 2, \ldots$, and $L_{1}(\nu):=$ $F^{-1}\left(L_{2}(\nu)\right)$. Then $L_{2}(0)=L_{2} ; \quad L_{1}(0)=L_{1}$, and the lines $\left\{L_{1}(\nu)\right\}_{\nu=0, \pm 1, \pm 2, \ldots}$ are parallel translates of $L_{1}$ in $\mathbb{R}_{x_{1}} \times \mathbb{R}_{x_{2}}$ by integer multiples of the vector $\left(d^{*}, 0\right)$ for some $d^{*}>0$. We note that each line $L_{2}(\nu)$ defines the same closed curve $l_{2}$ in $T_{2}$, and each $L_{1}(\nu)$ defines a closed curve $l_{1}(\nu)$ in $T_{1}$, but the curves $l_{1}(\nu)$ for distinct $\nu$ may be different. We note also that

$$
L_{1}(\nu) \times L_{2}(\nu) \subset \bar{H} .
$$

To verify this, fix a point $\left(x_{1}^{0}, x_{2}^{0}\right) \in L_{1}(\nu)$ so that $F\left(x_{1}^{0}, x_{2}^{0}\right) \in L_{2}(\nu)$. Since $L_{1}(\nu)=\left(x_{1}^{0}, x_{2}^{0}\right)+L_{1}$ and $L_{2}(\nu)=F\left(x_{1}^{0}, x_{2}^{0}\right)+L_{2}$, it follows that $L_{1}(\nu) \times L_{2}(\nu)=\left(\left(x_{1}^{0}, x_{2}^{0}\right)+L_{1}, F\left(x_{1}^{0}, x_{2}^{0}\right)+L_{2}\right) \in H+L_{1} \times L_{2}$. From 1., $H+L_{1} \times L_{2} \subset \bar{H}$ and the result follows. We thus have that

$$
\Lambda(z) \equiv \Lambda(\mathbf{0}) \quad \text { on } l_{1}(\nu) \times l_{2}(\nu), \nu=0, \pm 1, \ldots
$$

Our next goal is to show:

$(*)$ there exist unique $p, q \in \boldsymbol{Z}^{+}$with $(p, q)=1$ such that

$$
L_{1}(q)=L_{1}+p\left(\frac{1}{n}, 0\right) \text {, or, equivalently, } L_{2}+q\left(\frac{1}{n^{\prime}}, 0\right)=F\left(L_{1}+p\left(\frac{1}{n}, 0\right)\right) .
$$

We prove $(*)$ by contradiction. If $(*)$ is false, then writing $d^{*}=d_{0} \frac{1}{n}$, we see that $d_{0}$ must be irrational. Hence the set of curves $l_{1}(\nu), \nu= \pm 1, \pm 2, \ldots$ is dense in $T_{1}$. By the previous paragraph, $\Lambda \equiv \Lambda(\mathbf{0})$ on $T_{1} \times l_{2}$, which contradicts $\nabla \Lambda(\mathbf{0}) \neq \mathbf{0}$. Thus $(*)$ is proved.

For $s \in(-\infty, \infty)$, we define

$$
\begin{array}{ll}
\mathcal{L}_{1}(s):=L_{1}+p s(1 / n, 0) & \text { in } \mathbb{R}_{x_{1}} \times \mathbb{R}_{x_{2}} ; \\
\mathcal{L}_{2}(s):=L_{2}+q s\left(1 / n^{\prime}, 0\right) & \text { in } \mathbb{R}_{x_{3}} \times \mathbb{R}_{x_{4}}
\end{array}
$$

so that $\mathcal{L}_{2}(s)=F\left(\mathcal{L}_{1}(s)\right)$. Each line $\mathcal{L}_{1}(s)$ defines a simple closed curve $l_{1}(s)$ in $T_{1}$, and each line $\mathcal{L}_{2}(s)$ defines a simple closed curve $l_{2}(s)$ in $T_{2}$. Apriori, we define

$$
\Sigma:=\bigcup_{-\infty<s<\infty} l_{1}(s) \times l_{2}(s) \subset \mathbf{T} .
$$

However, $\mathcal{L}_{i}(s+k)=\mathcal{L}_{i}(s), i=1,2$ as a set in $T_{i}$, for all $k \in \boldsymbol{Z}$, so that

$$
\Sigma=\bigcup_{0 \leq s \leq 1} l_{1}(s) \times l_{2}(s) \subset \mathbf{T} .
$$


By the definition of $p, q \in \boldsymbol{Z}^{+}$we have $\left(\mathcal{L}_{1}(s) \times \mathcal{L}_{2}(s)\right) \cap\left(\mathcal{L}_{1}\left(s^{\prime}\right) \times \mathcal{L}_{2}\left(s^{\prime}\right)\right)=\emptyset$ for $s \neq s^{\prime}, 0 \leq s, s^{\prime} \leq 1$, so that

$$
\left(l_{1}(s) \times l_{2}(s)\right) \cap\left(l_{1}\left(s^{\prime}\right) \times l_{2}\left(s^{\prime}\right)\right)=\emptyset, \quad s \neq s^{\prime}, 0 \leq s<s^{\prime} \leq 1 .
$$

Then, since $l_{1}(s+1)=l_{1}(s), l_{2}(s+1)=l_{2}(s)$, and each $l_{1}(s), l_{2}(s)$ is a simple closed curve, it follows that $\Sigma$ is a three-dimensional, compact manifold in T.

We show that

$$
\Sigma=\bar{H}=H+L_{1} \times L_{2} \quad \text { in } T_{1} \times T_{2} .
$$

We proved in 1 . that $H+L_{1} \times L_{2} \subset \bar{H}$. We next show $\Sigma \subset H+L_{1} \times L_{2}$. Since

$$
\mathcal{L}_{2}(s)=F\left(\mathcal{L}_{1}(s)\right)=F\left(L_{1}+p s(1 / n, 0)\right)=L_{2}+F(p s(1 / n, 0)),
$$

it follows that

$$
\begin{aligned}
\mathcal{L}_{1}(s) \times \mathcal{L}_{2}(s) & =\left(\left(p s(1 / n, 0)+L_{1} ; F\left(p s(1 / n, 0)+L_{2}\right)\right.\right. \\
& =(p s(1 / n, 0) ; F(p s(1 / n, 0)))+\left(L_{1}, L_{2}\right) \in H+L_{1} \times L_{2} .
\end{aligned}
$$

Finally we show $H \subset \Sigma$; and hence $\bar{H} \subset \bar{\Sigma}=\Sigma$. Fix $\left(\left(x_{1}, x_{2}\right), F\left(x_{1}, x_{2}\right)\right) \in$ $H$. There is a unique $k \in \boldsymbol{Z}$ and $0 \leq s<1$ such that $\left(x_{1}, x_{2}\right) \in \mathcal{L}_{1}(k+s)$, or, equivalently, $\mathcal{L}_{1}(k+s)=\left(x_{1}, x_{2}\right)+L_{1}$. Then we have $F\left(x_{1}, x_{2}\right) \in \mathcal{L}_{2}(k+s)$, and hence $\mathcal{L}_{2}(k+s)=F\left(x_{1}, x_{2}\right)+L_{2}$. Since $\mathcal{L}_{1}(k+s)=\mathcal{L}_{1}(s)$ in $T_{1}$ and $\mathcal{L}_{2}(k+s)=\mathcal{L}_{2}(s)$ in $T_{2}$, it follows that

$$
\left(\left(x_{1}, x_{2}\right), F\left(x_{1}, x_{2}\right)\right) \in\left(\left(x_{1}, x_{2}\right)+L_{1}, F\left(x_{1}, x_{2}\right)+L_{2}\right)=\left(\mathcal{L}_{1}(s), \mathcal{L}_{2}(s)\right),
$$

which proves the inclusion $H \subset \Sigma$.

Equation (5.28) will lead us to the concrete formulas for the quotient space $\mathbf{T} / \bar{H}$ in $\mathbf{3}$. and the canonical projection $\widetilde{\pi}: \mathbf{T} \mapsto \mathbf{T} / \bar{H}$.

For $-\infty<t<\infty$, define

$$
\Sigma(t):=(p t(1 / n, 0) ; 0,0)+\Sigma \quad \text { in } \mathbf{T},
$$

which is a coset of $\Sigma$ and hence is a real three-dimensional compact manifold in $\mathbf{T}$. To be precise,

$$
\begin{aligned}
\Sigma(t) & =\bigcup_{0 \leq s \leq 1}\left(p t(1 / n, 0)+\mathcal{L}_{1}(s), \mathcal{L}_{2}(s)\right) /\left[e_{1}, e_{2}, e_{3}, e_{4}\right] \\
& =\bigcup_{0 \leq s \leq 1}\left(\mathcal{L}_{1}(t+s), \mathcal{L}_{2}(s)\right) /\left[e_{1}, e_{2}, e_{3}, e_{4}\right] \\
& =\bigcup_{0 \leq s \leq 1} l_{1}(t+s) \times l_{2}(s) .
\end{aligned}
$$


By (5.27), we have $\Sigma(t)=\Sigma\left(t^{\prime}\right)$ for $t-t^{\prime} \in \boldsymbol{Z}$ and $\Sigma(t) \cap \Sigma\left(t^{\prime}\right)=\emptyset$ for $t-t^{\prime} \notin \boldsymbol{Z}$. We shall show

$$
(* *) \quad \mathbf{T}=\bigcup_{0 \leq t \leq 1} \Sigma(t) .
$$

Note since $l_{1}(s)=l_{1}(s+1)$ in $T_{1}$, this is equivalent to (5.22). Fix $p_{0}=$ $\left(x_{1}, x_{2} ; x_{3}, x_{4}\right) /\left[e_{1}, e_{2}, e_{3}, e_{4}\right] \in \mathbf{T}$. Clearly we can choose real numbers $s_{1}, s_{2}$ so that $s_{1} p(1 / n, 0)$ is the $x_{1}$-intercept of the line $\left(x_{1}, x_{2}\right)+L_{1}$ and $s_{2} q\left(1 / n^{\prime}, 0\right)$ is the $x_{3}$-intercept of the line $\left(x_{3}, x_{4}\right)+L_{2}$. Since $\mathcal{L}_{1}\left(s_{1}\right)=\mathcal{L}_{1}\left(s_{2}\right)+t^{\prime} p(1 / n, 0)$ where $t^{\prime}=s_{1}-s_{2}$, it follows that

$$
\left(x_{1}, x_{2} ; x_{3}, x_{4}\right) \in\left(\mathcal{L}_{1}\left(s_{1}\right), \mathcal{L}_{2}\left(s_{2}\right)\right)=\left(\mathcal{L}_{1}\left(s_{2}\right), \mathcal{L}_{2}\left(s_{2}\right)\right)+\left(t^{\prime} p(1 / n, 0) ; 0,0\right),
$$

showing that $p_{0} \in \Sigma+\left(l_{2}\left(t^{\prime}\right) ; 0,0\right)=\Sigma\left(t^{\prime}\right)$. We now simply choose $n_{0} \in \boldsymbol{Z}$ and $t \in[0,1)$ so that $t^{\prime}=n_{0}+t$; then $\Sigma(t)=\Sigma\left(t^{\prime}\right)$, and hence $p_{0} \in \Sigma(t)$, proving $(* *)$ and thus 2 .(ii). Since $n_{0}, t$ are uniquely determined by the point $p_{0}=\left(x_{1}, x_{2}, x_{3}, x_{4}\right) /\left[e_{1}, e_{2}, e_{3}, e_{4}\right]$ in $\mathbf{T}$, it follows that

$$
\mathbf{T} / \bar{H} \approx \mathbb{R} /[1]=S^{1} \text { as real manifolds; }
$$

$\tilde{\pi}: \mathbf{T} \rightarrow \mathbf{T} / \bar{H}$ where $\tilde{\pi}\left(p_{0}\right)=p t(1 / n, 0)+\Sigma$, or equivalently, $\widetilde{\pi}\left(p_{0}\right)=t$.

Applying Remark 5.2 to our domain $D \subset \mathbf{T}$, there exist $0 \leq t_{1}<t_{2}<1$ such that $D=\cup_{t_{1}<t<t_{2}} \Sigma(t)$. Thus we have proved $\mathbf{3}$. The proofs of $\mathbf{1}$. and 2.(i) are elementary calculations. Note that $F\left(L_{1}\right)=L_{2} ; L_{1}$ is the line in $\mathbb{R}_{x_{1}} \times \mathbb{R}_{x_{2}}$ of slope $n / m \neq 0, \infty$ passing through the origin; and $L_{2}$ is the line in $\mathbb{R}_{x_{3}} \times \mathbb{R}_{x_{4}}$ of slope $n^{\prime} / M^{\prime} \neq 0, \infty$ passing through the origin. It follows from (5.16) that

$$
\frac{C-A(n / m)}{A+B(n / m)}=\frac{n^{\prime}}{M^{\prime}} .
$$

Furthermore, since $F\left(\mathcal{L}_{1}\right)=\mathcal{L}_{2}(1)$, the point $F(p / n, 0)$ lies on the line $\mathcal{L}_{2}(1)$ : $x_{4}=\frac{n^{\prime}}{M^{\prime}}\left(x_{3}-\frac{q}{n^{\prime}}\right)$. Hence

$$
\frac{C p}{n}=\frac{n^{\prime}}{M^{\prime}}\left(\frac{A p}{n}-\frac{q}{n^{\prime}}\right) .
$$

From these two equations and the Jacobian condition $A^{2}+B C+1=0$, a simple calculation yields

$$
A=\frac{p_{1} p_{2}+q_{1} q_{2}}{p_{2} q_{1}-p_{1} q_{2}}, \quad B=-\frac{p_{1}^{2}+q_{1}^{2}}{p_{2} q_{1}-p_{1} q_{2}}, \quad C=\frac{p_{2}^{2}+q_{2}^{2}}{p_{2} q_{1}-p_{1} q_{2}},
$$

where $p_{1}, p_{2}, q_{1}, q_{2}$ are defined by (5.18). Then we have

$$
b=-1 / B=\frac{p_{2} q_{1}-p_{1} q_{2}}{p_{1}^{2}+q_{1}^{2}}, \quad a=b A=\frac{p_{1} p_{2}+q_{1} q_{2}}{p_{1}^{2}+q_{1}^{2}},
$$


which proves (5.17) and hence 1.(i). By direct calculation

$$
F(m, n)=(A m+B n, C m-A n)=\frac{p}{q}\left(M^{\prime}, n^{\prime}\right),
$$

so that $\left(q(m, n), p\left(M^{\prime}, n^{\prime}\right)\right)$ lies on the integral curve $S$ in (5.14). We also have

$$
F(1 / n, 0)=(A / n, C / n)=\frac{q}{p}\left(1 / n^{\prime}, 0\right)+\frac{C}{n n^{\prime}}\left(M^{\prime}, n^{\prime}\right) .
$$

Since $((p / n, 0), F(p / n, 0)) \in S$, we have $\left(p(1 / n, 0), q\left(1 / n^{\prime}, 0\right)+\eta\left(M^{\prime}, n^{\prime}\right)\right) \in$ $S$, where $\eta=\frac{p C}{n n^{\prime}}=\frac{p}{n n^{\prime}} \frac{p_{2}^{2}+q_{2}^{2}}{p_{2} q_{1}-p_{1} q_{2}}$. This proves 1 .(ii).

Since $F(p / n, 0)=(A p / n, C p / n)$, using $F(0,0)=(0,0)$ it follows from the linearity of $F$ that $\left(x_{1}, 0 ; A x_{1}, C x_{1}\right) \in H$. This together with

$$
\left(L_{1}, 0,0\right),\left(0,0, L_{2}\right) \subset \bar{H}
$$

implies that the real Lie subalgebra $\mathfrak{h}_{0}$ of $\mathfrak{h}$ corresponding to the real threedimensional Lie subgroup $\bar{H}$ of $\mathbf{T}$ is generated by $\left\{X_{1}, X_{2}, X_{3}\right\}$ in $\mathfrak{g}$ where

$$
X_{1}=m \frac{\partial}{\partial x_{1}}+n \frac{\partial}{\partial x_{2}}, \quad X_{2}=M^{\prime} \frac{\partial}{\partial x_{3}}+n^{\prime} \frac{\partial}{\partial x_{4}}, \quad X_{3}=\frac{\partial}{\partial x_{1}}+A \frac{\partial}{\partial x_{3}}+C \frac{\partial}{\partial x_{4}} \text {. }
$$

Substituting $A$ and $C$ in the above formulas, we have by simple calculation that $\left\{q X_{1}, p X_{2},\left(p_{1}^{2}+p_{2}^{2}\right) X_{3}\right\}$ is equal to (5.19). Thus 2 . is proved.

It remains to prove 4 . Given integers $m, n, m^{\prime}, n^{\prime}, p, q$ as in 4 , we define $a, b$ by (5.17). Using the same method as in the above proof, we construct $D$ of the form (5.23). Since each $\Sigma(t)$ is a smooth, closed, Levi flat surface in $\mathbf{T}$ and $\Sigma(t) \Subset D$ for $t_{1}<t<t_{2}$, it follows that $D \subset \mathbf{T}$ is a pseudoconvex domain with smooth boundary which is not Stein. Moreover, there are no nonconstant holomorphic functions on $D$. To verify this, we may asume $\mathbf{0} \in D$. Suppose $f$ is a holomorphic function on $D$. Since $H$ is an analytic curve which is relatively compact in $D, f \equiv c$ on $H$. Hence $f \equiv c$ on $\bar{H}=\Sigma(0)=\Sigma$. Since $\Sigma$ is a real three-dimensional manifold, $f \equiv c$ on $D$.

Remark 5.4. The explicit formulas (5.29) for $a$ and $b$ in terms of $m, n, m^{\prime} \in$ $\boldsymbol{Z}, n^{\prime}, p, q \in \boldsymbol{Z}^{+}$and hence the explicit formulas involving the mapping $F:\left(x_{1}, x_{2}\right) \mapsto\left(x_{3}, x_{4}\right)$ imply the following results.

1. Recall that $\widehat{\mathcal{P}}:=\left\{F(i, j)+(K, l) \in \mathbb{R}_{x_{3}} \times \mathbb{R}_{x_{4}}: i, j, k, l \in \boldsymbol{Z}\right\}$ where $K=k+l \xi$. We showed that $C l[\widehat{\mathcal{P}}]$ consists of the family of parallel lines $L_{2}^{\nu}, \quad \nu=0, \pm 1, \pm 2, \ldots$, where $L_{2}^{\nu}=L_{2}+\nu(d, 0)$ and $L_{2}=\left\{t\left(M^{\prime}, n^{\prime}\right) \in\right.$ $\left.\mathbb{R}_{x_{3}} \times \mathbb{R}_{x_{4}}: t \in \mathbb{R}\right\}$. We shall show that $d=\frac{1}{p n^{\prime}}$. Fix $F(i, j)+(K, l) \in \widehat{\mathcal{P}}$. From our previous calculations, we have

$$
F(m, n)=\frac{p}{q}\left(M^{\prime}, n^{\prime}\right) \quad \text { and } \quad F(1 / n, 0)=\frac{q}{p}\left(1 / n^{\prime}, 0\right)+\eta\left(M^{\prime}, n^{\prime}\right)
$$


where $\eta$ is irrational. Since

$$
(i, j)=\frac{j}{n}(m, n)+(n i-j m)(1 / n, 0), \quad(K, l)=\frac{l}{n^{\prime}}\left(M^{\prime}, n^{\prime}\right)+\left(n^{\prime} k-m^{\prime} l\right)\left(1 / n^{\prime}, 0\right),
$$

it follows that

$$
\begin{aligned}
& F(i, j)+(K, l)=\frac{j p}{n q}\left(M^{\prime}, n^{\prime}\right)+(n i-j m)\left(\frac{q}{p}\left(1 / n^{\prime}, 0\right)+\eta\left(M^{\prime}, n^{\prime}\right)\right) \\
&+\frac{l}{n^{\prime}}\left(M^{\prime}, n^{\prime}\right)+\left(n^{\prime} k-m l\right)\left(1 / n^{\prime}, 0\right) \\
&=t\left(M^{\prime}, n^{\prime}\right)+\nu\left(\frac{1}{p n^{\prime}}, 0\right),
\end{aligned}
$$

where $t=\frac{j p}{n q}+\frac{l}{n^{\prime}}+(n i-j m) \eta \in \mathbb{R}$ and $\nu=(n i-j m) q+\left(n^{\prime} k-m^{\prime} l\right) p \in \boldsymbol{Z}$. This shows that $\widehat{\mathcal{P}} \subset L_{1}+\cup_{\nu=-\infty}^{\infty} \nu\left(\frac{1}{p n^{\prime}}, 0\right)$, so that $d / \frac{1}{p n^{\prime}}$ is a positive integer. On the other hand, in the above proof, from $(p, q)=1,(m, n)= \pm 1$ and $\left(m^{\prime}, n^{\prime}\right)= \pm 1$ we can choose $i, j, k, l \in \boldsymbol{Z}$ such that $\nu=1$. Hence $\frac{1}{p n^{\prime}} / d$ is an integer, so that $d=\frac{1}{p n^{\prime}}$.

2. Let

$$
\Delta_{1}:(0,0),\left(m_{1}, n_{1}\right),(m, n),\left(m_{1}+m, n_{1}+n\right)
$$

and

$$
\Delta_{2}:(0,0),\left(M_{1}^{\prime}, n_{1}^{\prime}\right),\left(M^{\prime}, n^{\prime}\right),\left(M_{1}^{\prime}+M^{\prime}, n_{1}^{\prime}+n^{\prime}\right)
$$

be fundamental domains associated to $L_{1}$ and $L_{2}$ in $\mathbb{R}_{x_{1}} \times \mathbb{R}_{x_{2}}$ and $\mathbb{R}_{x_{3}} \times \mathbb{R}_{x_{4}}$. Since $(m, n)=\left(\frac{1}{n}, 0\right)+\frac{n_{1}}{n}(m, n)$, we obtain from (5.30)

$$
\begin{aligned}
F(q(m, n)) & =p\left(M^{\prime}, n^{\prime}\right), \\
F\left(p\left(m_{1}, n_{1}\right)\right) & =q F\left(\frac{1}{n}, 0\right)+\frac{p n_{1}}{n} F(m, n)=q\left(\frac{1}{n^{\prime}}, 0\right)+\eta^{\prime \prime}\left(M^{\prime}, n^{\prime}\right),
\end{aligned}
$$

where $\eta^{\prime \prime}=\frac{p^{2} n_{1}}{n q}+p n$, so that $\eta^{\prime \prime}$ is irrational. 

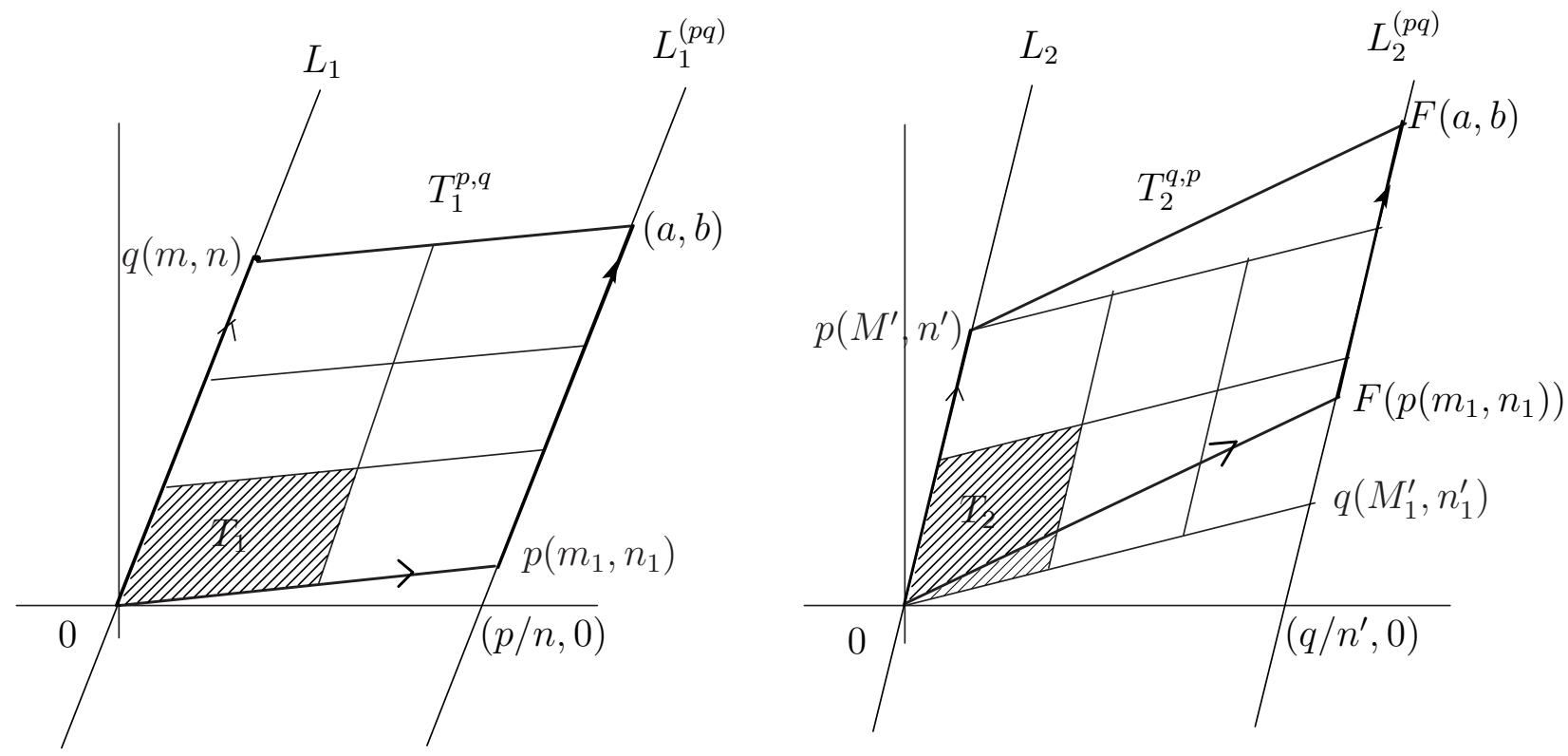

where $(a, b)=\left(q m+p m_{1}, q n+p n_{1}\right)$

Recall from Remark 5.4 that $l_{1}, l_{1}^{*}$ and $l_{2}, l_{2}^{*}$ are simple closed curves in $T_{1}$ and $T_{2}$. The set $T_{1}^{p, q}$ is the $p q$-sheeted torus over $T_{1}$ winding $p$ times along $l_{1}^{*}$ and $q$ times along $l_{1}$, while $T_{2}^{q, p}$ is the $p q$-sheeted torus over $T_{2}$ winding $q$ times along $l_{2}^{*}$ and $p$ times along $l_{2}$ (see the figure for the case $p=2, q=3$ ). This gives a visual interpretation of the Lie subgroup $H$ of $\mathbf{T}$ and its closure $\bar{H}$.

The topic of Levi flat surfaces in two dimensional complex tori has recently been studied by T. Ohsawa [16]. See also O. Suzuki [18].

\section{Complex homogeneous spaces.}

In this section, we let $M$ be an $n$-dimensional complex space with the property that there exists a connected complex Lie group $G \subset$ Aut $M$ of complex dimension $m \geq n$ which acts transitively on $M$. As prototypical examples, we can take $M=\mathbb{P}^{n}$ (complex projective space) and $G=G L(n+1, \mathbb{C}$ ), or more generally, we can take $M=G(k, n)$ (complex Grassmannian manifold) or $M=\mathcal{F}_{n}$ (complex flag space), and $G=G L(n, \mathbb{C})$. As noted in the previous section, another example is provided by $M=\mathbb{C}$ in which case $G=$ Aut $M$ is a noncommutative complex Lie group of complex dimension 2 .

We summarize some well-known results concerning homogeneous spaces which will be used in this section. We write $e$ for the identity element of $G$. 
1. For a fixed $z \in M$, let

$$
H_{z}:=\{g \in G: g(z)=z\}
$$

be the isotropy subgroup of $G$ for $z$. Then $H_{z}$ is a complex $(m-n)$ dimensional analytic set in $G$ without singular points. In particular, $H_{z}$ is closed and hence is a Lie subgroup of $G$, but $H_{z}$ is not always connected. We let $G / H_{z}$ denote the set of all left cosets $g H_{z}$. This quotient space $G / H_{z}$ has the structure of a complex $n$-dimensional manifold; moreover, if we let $\pi_{z}: G \mapsto G / H_{z}$ be the coset mapping $\pi_{z}(g)=g H_{z}$ and we let $\psi_{z}: G \mapsto M$ be the mapping $\psi_{z}(g)=g(z)$, then there exists an isomorphism $\alpha_{z}: G / H_{z} \rightarrow M$ such that $\alpha_{z} \circ \pi_{z}=\psi_{z}$ on $G$. This is equivalent to $\alpha_{z}\left(g H_{z}\right)=g(z)$ for $g \in G$. These three mappings are holomorphic; hence $\psi_{z}$ gives a holomorphic fibering of $G$ over $M$ with fibers $\psi_{z}^{-1}(\zeta) \approx H_{z}$ as complex manifolds; and, given $g \in G, \psi_{z}^{-1}(g(z))=g H_{z}$ as sets in $G$.

2. Fix $z \in M$ as in 1. Let $g_{0} \in G$ and set $z_{0}=g_{0}(z) \in M$. Then there exists a local holomorphic section $\sigma$ of $G$ with respect to $\psi_{z}$ over a neighborhood $V \subset M$ of $z_{0}$ passing through $g_{0}$; i.e.,

$$
\sigma\left(z_{0}\right)=g_{0} \quad \text { and } \quad \psi_{z} \circ \sigma=\text { identity on } V
$$

so that $\sigma(\zeta)(z)=\zeta$ for $\zeta \in V$. If we set $\tau: g \in \psi_{z}^{-1}(V) \mapsto \sigma(g(z))^{-1} g \in H_{z}$, then $\tau(g)$ depends holomorphically on $g$ and, for a fixed $z^{\prime} \in V$, the restriction of $\tau$ to $\psi_{z}^{-1}\left(z^{\prime}\right)$ is a bijection from $\psi_{z}^{-1}\left(z^{\prime}\right)$ onto $H_{z}$. It follows that

$$
T_{z}: g \in \psi_{z}^{-1}(V) \rightarrow(g(z), \tau(g)) \in V \times H_{z}
$$

provides a local trivialization of $G$ over $M$ with $T_{z}: \sigma(\zeta) \rightarrow(\zeta, e), \zeta \in V$. Similarly we have the following result. Fix $z, w \in M$ and $g \in G$ with $g(z)=w$. There exists a neighborhood $V$ of $z$ in $M$ and for each $\zeta \in V$, there exists an element $g_{\zeta} \in G$ such that $g_{z}=g ; g_{\zeta}(z)=w$ for $\zeta \in V$; and $g_{\zeta}$ depends holomorphically on $\zeta \in V$.

3. Fix $z_{0}, z_{1} \in M$. Let $h\left(z_{1}\right)=z_{0}$ where $h \in G$, so that $h^{-1} H_{z_{0}} h=H_{z_{1}}$. We then have two fiberings of $G$ over $M$ with respect to $\psi_{z_{0}}$ and $\psi_{z_{1}}$. We consider the right translation $R_{h}: G \rightarrow G$ where $R_{h}(g)=g h, g \in G$. Then $R_{h}$ induces a holomorphic isomorphism $\mathcal{R}_{h}: G / H_{z_{0}} \rightarrow G / H_{z_{1}}$ via $\mathcal{R}_{h}\left(g H_{z_{0}}\right)=g h H_{z_{1}}, g \in G$. In other words, for $z \in M$, take any $g, k \in G$ with $z=g\left(z_{0}\right)=k\left(z_{1}\right)$. Then $\mathcal{R}_{h}\left(g H_{z_{0}}\right)=k H_{z_{1}}$.

Let $D \Subset M$ be a domain with $C^{\infty}$ boundary in $M$. For $z \in M$, we let

$$
D(z):=\{g \in G: g(z) \in D\}=\psi_{z}^{-1}(D)=\pi_{z}^{-1} \circ \alpha_{z}^{-1}(D)
$$

be a possibly unbounded and possibly disconnected domain in $G$. Thus 


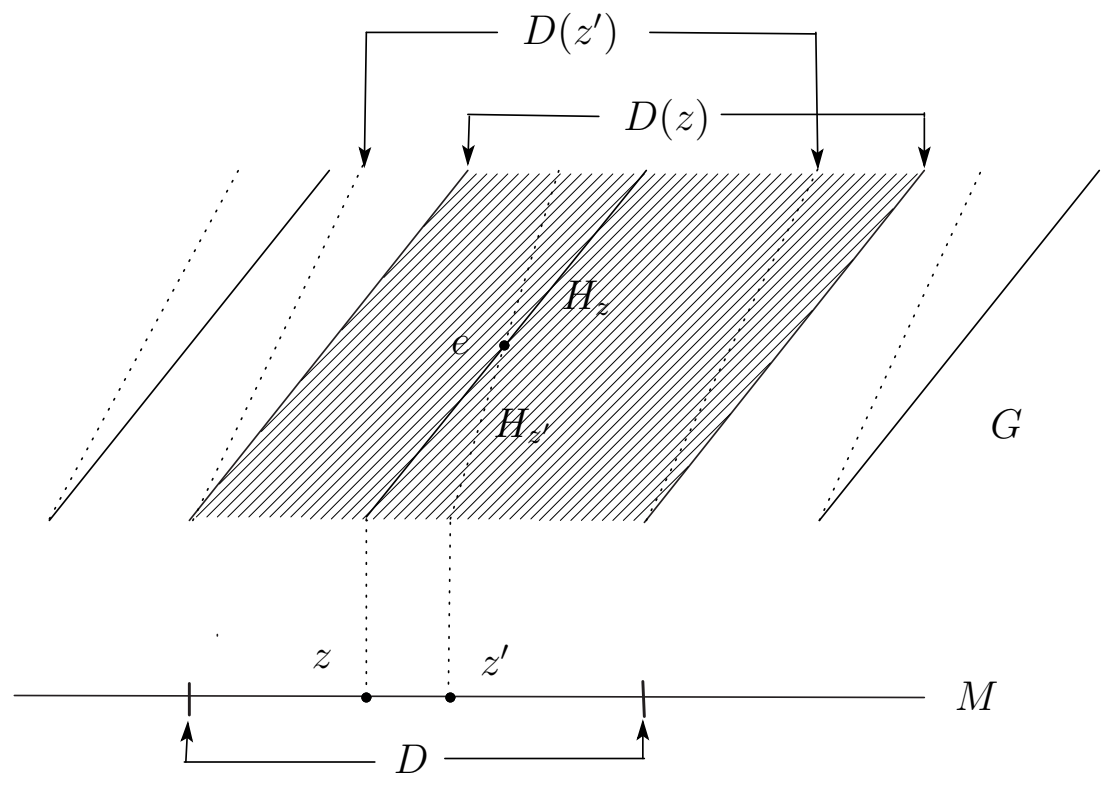

$D(z)$ is a set of cosets modulo $H_{z}$ in $G$, i.e., $D(z)$ may be considered as a subset of $G / H_{z}$ in such a way that $\alpha_{z}$ maps $D(z) / H_{z}$ biholomorphically to $D$. We remark that the connectedness or disconnectedness of $D(z)$ in $G$ is independent of the point $z \in M$. Indeed, given $z^{\prime} \in M$, take $h \in G$ such that $h(z)=z^{\prime}$. Since the equality $D(h(z))=D(z) h^{-1}$ holds (this will be proved as II. in Proposition [6.2), we have $D\left(z^{\prime}\right)=D(z) h^{-1}$, so that $D(z)$ and $D\left(z^{\prime}\right)$ are simultaneously connected or disconnected in $G$. Note that by homogeneity we have, for any $z \in M$,

$$
D=\{g(z) \in M: g \in D(z)\} .
$$

We also mention that in the case when the isotropy subgroup $H_{z}$ of $G$ is connected, $D(z)$ is connected in $G$. For, we may assume $z \in D$, so that $e \in D(z)$ and $H_{z} \subset D(z)$. Let $g \in D(z)$, i.e., $g(z) \in D$. We take a continuous curve $\gamma: t \in[0,1] \rightarrow \gamma(t)$ in $D$ such that $\gamma(0)=g(z)$ and $\gamma(1)=z$. We use property 2. to piece together a continuous section $\sigma: t \in[0,1] \rightarrow \sigma(t)$ of $G$ over $\gamma$ via $\psi_{z}$, i.e., $\sigma(t)(z)=\gamma(t)$ for $t \in[0,1]$, with $\sigma(0)=g$. Since $\gamma \subset D$ and $\sigma(0) \in D(z)$, we have $\sigma \subset D(z)$. Also, since $\sigma(1)(z)=z$, we have $\sigma(1) \in H_{z}$. From the assumption that $H_{z}$ is connected, we can find a continuous curve $\eta: t \in[0,1] \rightarrow \eta(t)$ in $H_{z}$ such that $\eta(0)=\sigma(1)$ and $\eta(1)=e$. Putting together the curves $\sigma$ and $\eta$; i.e., defining the concatenation

$$
\Gamma(t):=\sigma(2 t) \text { for } t \in[0,1 / 2] ; \text { and } \Gamma(t)=\eta(2 t-1) \text { for } t \in[1 / 2,1],
$$

gives a continuous curve $\Gamma: t \in[0,1] \rightarrow \Gamma(t)$ in $G$ starting at $g$ and terminating at $e$ which lies entirely in $D(z)$. Hence $D(z)$ is a connected subset of 
$G$.

We next consider the case when $H_{z}$ is not connected in $G$. We write $H_{z}=\cup_{k=1}^{\infty} H_{z}^{(k)}$ as the union of its connected components in $G$. We let $H_{z}^{\prime}=H_{z}^{(1)}$ denote the connected component of $H_{z}$ which contains the identity e. Note that $\left\{H_{z}^{(k)}\right\}_{k}$ are isolated sets in $G$ (see 1. in Proposition 6.4). If we take $h_{k} \in H_{z}^{(k)}, k=1,2, \ldots$, then

$$
H_{z}^{(k)}=h_{k} H_{z}^{\prime}=H_{z}^{\prime} h_{k}, \quad k=1,2, \ldots
$$

To see this, since $h_{k} H_{z}^{\prime}$ is connected in $G$ and contains $h_{k}$, it follows from the inclusion $h_{k} H_{z}^{\prime} \subset H_{z}$ that $h_{k} H_{z}^{\prime} \subset H_{z}^{(k)}$. Conversely, since $h_{k}^{-1} H_{z}^{(k)}$ is connected in $G$, it follows from $e \in h_{k}^{-1} H_{z}^{(k)} \subset H_{z}$ that $h_{k}^{-1} H_{z}^{(k)} \subset H_{z}^{\prime}$. Thus the first equality holds. The second one can be verified in a similar fashion; it also follows from the first by the normality of $H_{z}^{\prime}$ in $H_{z}$.

In the case when $H_{z}$ is not connected the connectivity of $D(z)$ depends on the domain $D$ in $M$. In fact, if $D$ is very small and $z \in D$, then each connected component of $D(z)$ contains only one component of $H_{z}$. For a general domain $D \Subset M$ and $z \in D$, we decompose $D(z)$ into a finite or a countably infinite collection of connected components:

$$
D(z)=\bigcup_{i=1}^{\infty} D^{(i)}(z) .
$$

We write $D^{\prime}(z):=D^{(1)}(z)$ for the connected component which contains the identity $e$, so that $H_{z}^{\prime} \subset D^{\prime}(z)$. We set $\mathcal{H}^{\prime}(z):=H_{z} \cap D^{\prime}(z)$. Then $\mathcal{H}^{\prime}(z)$ consists of a finite or a countably infinite collection of components $\left\{H_{z}^{(k)}\right\}_{k=1}^{\infty}$, say

$$
\mathcal{H}^{\prime}(z)=\left\{H_{z}^{\prime}, H_{z}^{\left(\alpha_{2}\right)}, H_{z}^{\left(\alpha_{3}\right)}, \ldots\right\}=\bigcup_{j=1}^{\infty} h_{j}^{\prime} H_{z}^{\prime}
$$

where $h_{1}^{\prime}=e$ and $h_{j}^{\prime} \in H_{z}^{\left(\alpha_{j}\right)}, j=2,3, \ldots$ (using (6.3) ). We have that $g \in D^{\prime}(z)$ implies that $g \mathcal{H}^{\prime}(z) \subset D^{\prime}(z)$. To see this, let $h \in \mathcal{H}^{\prime}(z)$. We connect $e$ and $g$ by a continuous curve $\gamma: t \in[0,1] \rightarrow \gamma(t)$ in $D^{\prime}(z)$. Then the continuous curve $\widetilde{\gamma}: t \in[0,1] \rightarrow \gamma(t) h$ in $G$ connects $h$ and $g h$. Since $\psi_{z}(\widetilde{\gamma})=\psi_{z}(\gamma) \subset D$ and $\widetilde{\gamma}(0)=h \in \mathcal{H}^{\prime}(z) \subset D^{\prime}(z)$, we have $\widetilde{\gamma} \subset D^{\prime}(z)$, and hence $g h=\widetilde{\gamma}(1) \in D^{\prime}(z)$.

Next we show that for each $i \geq 2$ we have $D^{(i)}(z) \cap H_{z} \neq \emptyset$. We begin with $g_{0} \in D^{(i)}(z)$ and set $z_{0}:=g_{0}(z) \in D$. Take a continuous curve $\gamma: t \in[0,1] \rightarrow$ $\gamma(t)$ in $D$ such that $\gamma(0)=z_{0}$ and $\gamma(1)=z$. Using property 2., we can construct a continuous section $\sigma: t \in[0,1] \rightarrow \sigma(t)$ of $G$ over $\gamma$ with respect to $\psi_{z}$ such that $\sigma(0)=g_{0}$. Hence $\sigma \subset D(z)$. Then $\sigma(0)=g_{0} \in D^{(i)}(z)$ implies that $\sigma \subset D^{(i)}(z)$. On the other hand, since $\sigma(1)(z)=\gamma(1)=z$, we have $\sigma(1) \in H_{z}$, so that $\sigma(1) \in H_{z} \cap D^{(i)}(z)$. 
Thus for $i=2, \ldots$ we define the non-empty sets $\mathcal{H}^{(i)}(z):=D^{(i)}(z) \cap H_{z}$ and we can write

$$
H_{z}=\bigcup_{i=1}^{\infty} \mathcal{H}^{(i)}(z)
$$

where $\mathcal{H}^{(1)}=\mathcal{H}^{\prime}(z)$ and the union is disjoint. If we fix $\mathbf{h}^{(i)} \in \mathcal{H}^{(i)}(z), i=$ $2,3, \ldots$, then we have

$$
D^{(i)}(z)=D^{\prime}(z) \mathbf{h}^{(i)}, \quad \mathcal{H}^{(i)}(z)=\mathcal{H}^{\prime}(z) \mathbf{h}^{(i)} .
$$

To verify these equalities, first observe that since $D^{\prime}(z) \mathbf{h}^{(i)}$ is a connected subset of $D(z)$ which contains $\mathbf{h}^{(i)}$, we have $D^{\prime}(z) \mathbf{h}^{(i)} \subset D^{(i)}(z)$. A similar argument implies that $D^{(i)}(z)\left(\mathbf{h}^{(i)}\right)^{-1} \subset D^{\prime}(z)$, so that the first formula holds. An interpretation of this formula is that each $D^{(i)}(z), i=2,3, \ldots$ is isomorphic to $D^{\prime}(z)$ via right translation $\mathcal{R}_{\mathbf{h}_{i}}: g \in D^{\prime}(z) \rightarrow g \mathbf{h}^{(i)} \in D^{(i)}(z)$. The second formula clearly follows from the first and the definitions of $\mathcal{H}^{(i)}(z)$ and $\mathcal{H}^{\prime}(z)$.

Proposition 6.1. Let $z \in D$. Then

1. $D=\left\{g(z) \in M: g \in D^{\prime}(z)\right\}$;

2. $D^{\prime}(z) h \subset D^{\prime}(z)$ for $h \in \mathcal{H}^{\prime}(z)$;

3. let $g \in D^{\prime}(z)$ and $h \in H_{z}$. If $g h \in D^{\prime}(z)$, then $h \in \mathcal{H}^{\prime}(z)$.

Proof. To prove 1., we have from (6.2) that

$$
\left\{g(z) \in M: g \in D^{\prime}(z)\right\} \subset D .
$$

For the reverse inclusion, fix $\hat{z} \in D$ and consider a continuous curve $\gamma: t \in$ $[0,1] \rightarrow \gamma(t)$ in $D$ such that $\gamma(0)=z$ and $\gamma(1)=\hat{z}$. By property 2., we can find a continuous section $\sigma: t \in[0,1] \rightarrow \sigma(t)$ of $G$ over $\gamma$ via $\psi_{z}$ such that $\sigma(0)=e$. Consequently, since $\sigma(0)=e \in D^{\prime}(z)$, we have $\sigma \subset D^{\prime}(z)$. In particular, $\sigma(1) \in D^{\prime}(z)$ and $\sigma(1)(z)=\gamma(1)=\hat{z}$, so that taking $g:=\sigma(1)$ verifies the reverse inclusion. To prove 2 ., let $g \in D^{\prime}(z)$ and $h \in \mathcal{H}^{\prime}(z)$. We take a continuous curve $\eta: t \in[0,1] \rightarrow \eta(t)$ in $D^{\prime}(z)$ such that $\eta(0)=e$ and $\eta(1)=g$. Then the continuous curve $\xi: t \in[0,1] \rightarrow \xi(t):=\eta(t) h$ in $G$ satisfies $\xi(t)(z)=\eta(t) h(z)=\eta(t)(z) \in D, t \in[0,1]$. It follows from $\xi(0)=h \in D^{\prime}(z)$ that $\xi(t) \in D^{\prime}(z), t \in[0,1]$, and hence $g h=\xi(1) \in D^{\prime}(z)$, which proves 2 . To prove (3), we set $g^{\prime}=g h \in D^{\prime}(z)$. Since $g \in D^{\prime}(z)$ we can find a continuous curve $\tau: t \in[0,1] \rightarrow g(t)$ in $D^{\prime}(z)$ such that $g(0)=e$ and $g(1)=g$. Consider the continuous curve $\tau^{\prime}: t \in[0,1] \rightarrow \tau^{\prime}(t):=g(t) h$ in $G$. We have $\tau^{\prime}(t)(z)=g(t) h(z)=g(t)(z) \in D$. Since $\tau^{\prime}(1)=g h \in D^{\prime}(z)$, 
it follows that $\tau^{\prime} \subset D^{\prime}(z)$. In particular, $h=e h=\tau^{\prime}(0) \in D^{\prime}(z)$, which proves (3).

We remark that we can rephrase 1 . of Proposition 6.1 as $\left[D^{\prime}\left(z_{0}\right)\right]\left(z_{0}\right)=D$. In a similar fashion, it can be shown that

$$
\left[\partial D^{\prime}\left(z_{0}\right)\right]\left(z_{0}\right)=\partial D
$$

where $\partial D^{\prime}\left(z_{0}\right)$ is the boundary of $D^{\prime}\left(z_{0}\right)$ in $G$ and $\partial D$ is the boundary of $D$ in $M$.

Corollary 6.1. Let $z \in D$. Then $\mathcal{H}^{\prime}(z)$ is a closed Lie subgroup of $H_{z}$.

Proof. We first prove that $\mathcal{H}^{\prime}(z)$ is a subgroup of $H$. Since $e \in \mathcal{H}^{\prime}(z)$, it suffices to prove that $g:=h_{1} h_{2}^{-1} \in \mathcal{H}^{\prime}(z)$ for $h_{1}, h_{2} \in \mathcal{H}^{\prime}(z)$. Since $g(z)=z$, we have $g \in H_{z}$. Then using $h_{1}=g h_{2}$ and $h_{1} \in D^{\prime}(z)$, it follows from 3 . in Proposition 6.1 that $g \in \mathcal{H}^{\prime}(z)$. We next verify that $\mathcal{H}^{\prime}(z)$ is closed in $G$; since a closed subgroup of a Lie group is a Lie subgroup, this will complete the proof. Let $h_{n} \in \mathcal{H}^{\prime}(z), n=1,2, \ldots$ and fix $g \in G$ with $h_{n} \rightarrow g(n \rightarrow \infty)$. Since $H_{z}$ is closed, we have $g \in H_{z}$. Since $e \in D^{\prime}(z), D^{\prime}(z)$ is open in $G$, and $g h_{n}^{-1} \rightarrow e$, it follows that $g h_{n}^{-1} \in D^{\prime}(z)$ for sufficiently large $n$. Thus $g \in D^{\prime}(z) h_{n}$. Using 2. in Proposition 6.1 we conclude that $g \in D^{\prime}(z)$, and hence $g \in \mathcal{H}^{\prime}(z)$.

Proposition 6.2. We have

$$
\begin{aligned}
& \text { I. } e \in D(z)\left(\partial D(z), \overline{D(z)}^{c}\right) \text { if and only if } z \in D\left(\partial D, \bar{D}^{c}\right) . \\
& \text { II. } D(h(z))=D(z) h^{-1} \text { for } z \in M \text { and } h \in G . \\
& \text { III. } D^{\prime}(h(z))=D^{\prime}(z) h^{-1} \text { for } z \in D \text { and } h \in D^{\prime}(z) .
\end{aligned}
$$

Proof. By definition, we have $e \in D(z)$ if and only if $z \in D$. Let $e \in \partial D(z)$. Then there exists a sequence $\left\{g_{n}\right\}_{n} \subset D(z)$ such that $g_{n} \rightarrow e$. Hence, $g_{n}(z) \rightarrow z$ in $M$. Since $g_{n}(z) \in D$ and $z \notin D$, we have $z \in \partial D$. Conversely, let $z \in \partial D$. Then $e \notin D(z)$. We fix a holomorphic section $\sigma$ of $G$ via $\psi_{z}$ over a neighborhood $V \subset M$ of $z$ with $\sigma(z)=e$; thus $\sigma(\zeta)(z)=\zeta$ for all $\zeta \in V$. We take $\left\{\zeta_{n}\right\}_{n} \subset V \cap D$ such that $\zeta_{n} \rightarrow z$ as $n \rightarrow \infty$. It follows that $\sigma\left(\zeta_{n}\right) \in D(z)$ and $\sigma\left(\zeta_{n}\right) \rightarrow e$ as $n \rightarrow \infty$, so that $e \in \partial D(z)$. We thus have $e \in \partial D(z) \Leftrightarrow z \in \partial D$, which proves I.

Property II. is based on (6.2). Indeed, $g \in D(h(z))$ if and only if $g h(z) \in$ $D$ if and only if $g h \in D(z)$ if and only if $g \in D(z) h^{-1}$.

To prove III., we fix $z \in D$ and $h \in D^{\prime}(z)$. Then II. implies $D(h(z))=$ $D(z) h^{-1}$. From (6.4) we thus obtain $D(h(z))=\cup_{i=1}^{\infty}\left[D^{(i)}(z) h^{-1}\right]$ where this 
is a disjoint union. Hence $D^{\prime}(h(z))$ coincides with some $D^{(i)}(z) h^{-1}$ which contains the identity element $e$. On the other hand, since $h \in D^{\prime}(z)$, we have $e \in D^{\prime}(z) h^{-1}$; hence $i=1$ and $D^{\prime}(h(z))=D^{\prime}(z) h^{-1}$.

Let $\partial D$ denote the boundary of $D$ in $M$ and let $\partial D(z)$ denote the relative boundary of $D(z)$ in $G$. Note that $\partial D(z)=\psi_{z}^{-1}(\partial D)$ is smooth in $G$, but it is not necessarily compact in $G$. A similar argument to that used in proving I. shows that for $g \in G$ and $z \in M, g \in \partial D(z)$ if and only if $g(z) \in \partial D$. Moreover, we note that, for $z \in D$ and $h \in D^{\prime}(z)$,

$$
\partial D^{\prime}(h(z))=\left(\partial D^{\prime}(z)\right) h^{-1} \quad \text { and } \quad{\overline{D^{\prime}(h(z))}}^{c}=\left[{\overline{D^{\prime}(z)}}^{c}\right] h^{-1} .
$$

It suffices to prove the first formula. Let $g \in \partial D^{\prime}(h(z))$. Then $g \notin$ $D^{\prime}(h(z))$ and there exists $g_{n} \in D^{\prime}(h(z))$ such that $\lim _{n \rightarrow \infty} g_{n}=g$ in $G$. From III. there exists $k_{n} \in D^{\prime}(z)$ such that $g_{n}=k_{n} h^{-1}$. Thus, $k:=\lim _{n \rightarrow \infty} k_{n}=$ $g h$. Since $k=g h \notin D^{\prime}(h(z)) h=D^{\prime}(z)$ by III., it follows that $k \in \partial D^{\prime}(z)$, and hence $g=k h^{-1} \in\left(\partial D^{\prime}(z)\right) h^{-1}$, so that $\partial D^{\prime}(h(z)) \subset\left(\partial D^{\prime}(z)\right) h^{-1}$. To prove the reverse inclusion, let $g \in \partial D^{\prime}(z)$. Then $g h^{-1} \notin D^{\prime}(z) h^{-1}=D^{\prime}(h(z))$. We take $\left\{g_{n}\right\} \subset D^{\prime}(z), n=1,2, \ldots$ with $g_{n} \rightarrow g$ in $G$ as $n \rightarrow \infty$. Since $g_{n} h^{-1} \in D^{\prime}(z) h^{-1}=D^{\prime}(h(z))$, we have $g h^{-1} \in \partial D^{\prime}(h(z))$.

From II. we see that for $z, z^{\prime} \in M, D(z)$ and $D\left(z^{\prime}\right)$ are biholomorphically equivalent. In case $z, z^{\prime} \in D$, from III. and 1. in Proposition 6.1, $D^{\prime}(z)$ and $D^{\prime}\left(z^{\prime}\right)$ are also biholomorphically equivalent.

Lemma 6.1. Let $\zeta \in \partial D$ and let $\left\{z_{n}\right\}_{n} \subset D$ converge to $\zeta$ as $n \rightarrow \infty$. Then there exist $g_{n} \in \partial D^{\prime}\left(z_{n}\right), n=1,2, \ldots$, such that $g_{n} \rightarrow e$ in $G$ as $n \rightarrow \infty$.

Proof. We take a holomorphic section $\sigma: V \rightarrow G$ of the fiber space $\psi_{\zeta}: G \rightarrow$ $M$ over a neighborhood $V \subset M$ of $\zeta$ with $\sigma(\zeta)=e$. For sufficiently large $n$ we have $z_{n} \in V$ and hence $\sigma\left(z_{n}\right)(\zeta)=z_{n}$. It follows that $g_{n}:=\sigma\left(z_{n}\right)^{-1}$ is close to $e$ in $G$; moreover, we have $g_{n} \in \partial D\left(z_{n}\right)$, for $g_{n}\left(z_{n}\right)=\sigma\left(z_{n}\right)^{-1} \sigma\left(z_{n}\right)(\zeta)=$ $\zeta \in \partial D$. Thus, $g_{n} \in \partial D^{\prime}\left(z_{n}\right)$.

Proposition 6.3. Assume that $D$ is pseudoconvex in $M$, and fix $z \in M$. Then we have

1. $D(z)$ is pseudoconvex in $G$; and

2. there exists a sequence of piecewise smooth pseudoconvex domains $\left\{D_{n}(z)\right\}_{n}$ in $G$ such that $D_{n}(z) \subset D_{n+1}(z) \Subset D(z)$ and $D(z)=\bigcup_{n=1}^{\infty} D_{n}(z)$.

Proof. Recall that $\partial D(z)=\psi_{z}^{-1}(\partial D)$ is smooth in $G$. Let $g_{0} \in \partial D(z)$ in $G$ and set $z_{0}=g_{0}(z)$ in $M$. Then $z_{0} \in \partial D$. By (6.1), we can find a neighborhood $V$ of $z_{0}$ in $M$ such that setting $\mathcal{V}=\psi_{z}^{-1}(V) \subset G$, we have 
$\mathcal{V} \cap \partial D(z)$ is biholomorphically equivalent to $(V \cap \partial D) \times H_{z}$. Noting that $H_{z}$ is an $(m-n)$-dimensional complex manifold, the pseudoconvexity of $\partial D$ at $z_{0}$ yields the same property for $\partial D(z)$ at $g_{0}$, proving 1.

To show 2., we need the following result of Kazama [7]: a complex Lie group $G$ is weakly complete: $G$ admits a $C^{\infty}$ plurisubharmonic exhaustion function; i.e., there exists a $C^{\infty}$ plurisubharmonic function $\varphi$ in $G$ with $\{g \in$ $G: \varphi(g)<\alpha\} \Subset G$ for each $\alpha \in \mathbb{R}$. Given $n \gg 1$, we set

$$
D_{n}(z):=D(z) \cap\{g \in G: \varphi(g)<n\} .
$$

By 1., we see that $D_{n}(z)$ is a pseudoconvex domain with piecewise smooth boundary in $G$; clearly $D_{n}(z) \subset D_{n+1}(z) \Subset D(z)$ and $D(z)=\cup_{n=1}^{\infty} D_{n}(z)$.

Now we discuss two types of variations of domains in $G$. First, we have a variation of domains $D(z)$ in $G$ with parameter $z \in M$ :

$$
\mathcal{D}_{M}: z \in M \rightarrow D(z) \subset G \text {. }
$$

As usual we identify the variation $\mathcal{D}_{M}$ with the $(m+n)$-dimensional domain $\mathcal{D}_{M}=\cup_{z \in M}(z, D(z)) \subset M \times G$. Given a domain $V \subset M$, we consider the variation $\mathcal{D}_{V}: z \in V \rightarrow D(z)$, and identify $\mathcal{D}_{V}$ with $\cup_{z \in V}(z, D(z)) \subset V \times G$.

Next we define $\mathcal{D}:=\cup_{z \in D}(z, D(z))$, a variation of domains $D(z) \subset G$ with parameter $z \in D$ :

$$
\mathcal{D}: z \in D \rightarrow D(z) \subset G .
$$

Furthermore we define $\mathcal{D}^{\prime}:=\cup_{z \in D}\left(z, D^{\prime}(z)\right)$, where recall that $D^{\prime}(z)$ is the connected component of $D(z)$ containing the identity $e$. This is a variation of domains $D^{\prime}(z) \subset G$ with parameter $z \in D$ :

$$
\mathcal{D}^{\prime}: z \in D \rightarrow D^{\prime}(z) \subset G .
$$

Lemma 6.2. $\mathcal{D}$ and $\mathcal{D}^{\prime}$ are locally holomorphically trivial variations.

Proof. Since the proofs are similar, we only give the proof of the lemma for $\mathcal{D}^{\prime}$. Let $z_{0} \in D$. By taking $z=z_{0}$ in property 2 ., we can find a neighborhood $V \subset D$ of $z_{0}$ and a holomorphic section $\sigma$ over $V$ via $\psi_{z_{0}}$ such that $\sigma(\zeta)\left(z_{0}\right)=$ $\zeta$ for $\zeta \in V$ with $\sigma\left(z_{0}\right)=e$. Since $\sigma(\zeta)\left(z_{0}\right)=\zeta \in V \subset D$, we have $\sigma(\zeta) \in D\left(z_{0}\right)$. It follows from $\sigma\left(z_{0}\right)=e \in D^{\prime}\left(z_{0}\right)$ that $\sigma(\zeta) \in D^{\prime}\left(z_{0}\right)$. By III in Proposition 6.2 we have $D^{\prime}\left(z_{0}\right)=D^{\prime}\left(\sigma(\zeta)^{-1}(\zeta)\right)=D^{\prime}(\zeta) \sigma(\zeta)$. Hence,

$$
T:(\zeta, g) \in \mathcal{D}_{V}^{\prime}:=\cup_{\zeta \in V}\left(\zeta, D^{\prime}(\zeta)\right) \rightarrow(\zeta, g \sigma(\zeta)) \in V \times D^{\prime}\left(z_{0}\right)
$$

provides a trivialization. 
Fix a Kähler metric $d s^{2}$ on $G$ (such a metric exists by [8]) and let $c$ be a strictly positive $C^{\infty}$ function on $G$. As shown in the previous section, for a fixed $z \in D$, since $e \in D(z)$, we can form the $c$-Robin constant $\lambda(z)$ for $(D(z), e)$. We recall that the $c$-Robin constant is defined by the usual exhaustion method in the case of an unbounded connected domain $D(z)$ (see, for example, Chapter IV in [2]). Furthermore, if $D(z)$ is not connected, we consider the $c$-Robin constant $\lambda(z)$ for $\left(D^{\prime}(z), e\right)$ and we call this the $c$-Robin constant for $(D(z), e)$. Using standard methods from potential theory, from Lemma 6.2 we see that $\lambda(z)$ is smooth in $D$. Furthermore, since $\partial D$ is smooth in $G$, Lemma 6.1 implies that $\lambda(z) \rightarrow-\infty$ as $z \rightarrow \partial D$; i.e., $-\lambda(z)$ is a smooth exhaustion function for $D$. We call $\lambda(z)$ the $c$-Robin function for $D$. Under these circumstances we have the following result.

Theorem 6.1. If $D \Subset M$ is a smoothly bounded pseudoconvex domain, then the $c$-Robin function $-\lambda(z)$ for $D$ is a plurisubharmonic exhaustion function for $D$.

Proof. It remains to prove the plurisubharmonicity of $-\lambda(z)$ on $D$. Using $D_{n}(z) \Subset G$ from 2. of Proposition 6.3, we set $\mathcal{D}_{n}=\cup_{z \in D}\left(z, D_{n}(z)\right)$ and $\mathcal{D}_{n}^{\prime}=\cup_{z \in D}\left(z, D_{n}^{\prime}(z)\right)$ where $D_{n}^{\prime}(z)$ is the connected component of $D_{n}(z)$ containing $e$. By Lemma 6.2, $\mathcal{D}_{n}^{\prime}$ as well as $\mathcal{D}_{n}$ is pseudoconvex in $D \times G$. Let $V \Subset D$ be a domain. We take $n$ sufficiently large so that $e \in D_{n}(z)$ for $z \in V$. Then for $z \in V$ we may consider the $c$-Robin constant $\lambda_{n}(z)$ for $\left(D_{n}(z), e\right)$, i.e., for $\left(D_{n}^{\prime}(z), e\right)$. It follows from Theorem 3.2 that $-\lambda_{n}(z)$ is a plurisubharmonic function on $V$. (We remark that we assumed $\partial \mathcal{D}$ was smooth in this theorem; however, it is standard to see that the result remains true in the case $\partial \mathcal{D}$ is only assumed to be piecewise smooth.) Since $-\lambda_{n}(z)$ decreases to $-\lambda(z)$ in $V,-\lambda(z)$ is plurisubharmonic on $V$, and hence on $D$.

We next discuss conditions under which $-\lambda(z)$ is strictly plurisubharmonic on $D$. Suppose not; i.e., suppose there exists a point $z_{0} \in D$ at which the complex Hessian $\left[\frac{\partial^{2}(-\lambda)}{\partial z_{j} \partial \bar{z}_{k}}\left(z_{0}\right)\right]$ has a zero eigenvalue so that $\left.\left[\frac{\partial^{2}\left(-\lambda\left(z_{0}+a t\right)\right)}{\partial t \partial \bar{t}}\right]\right|_{t=0}$ $=0$ for some direction $a \in \mathbb{C}^{n}(a \neq 0)$. A standard result in the theory of homogeneous spaces is that $M \approx G / H_{z_{0}}$ via the map $\psi_{z_{0}}(g)=g\left(z_{0}\right)$; using this it follows that there exists a left-invariant holomorphic vector field $X$ on $G$ such that the tangent vector of $(\exp t X)\left(z_{0}\right)$ at $t=0$ in $M$ is equal to $a$; and thus $\left.\left[\frac{\partial^{2}\left(-\lambda\left(\exp t X\left(z_{0}\right)\right)\right.}{\partial t \partial \bar{t}}\right]\right|_{t=0}=0$.

Note that $\exp t X \in D^{\prime}\left(z_{0}\right)$ for $|t|<\rho$ if $\rho$ is sufficiently small. It follows from III. in Proposition 6.2 that

$$
D^{\prime}\left(\exp t X\left(z_{0}\right)\right)=D^{\prime}\left(z_{0}\right) \cdot(\exp t X)^{-1} .
$$


We thus apply Lemma 5.1 to $G, D^{\prime}\left(z_{0}\right)$, and $e$ corresponding to $M, D$, and $\zeta$ in the lemma - note that the domain $D$ in the lemma is bounded, but the argument is valid for unbounded $D$ - to obtain the following facts: if we assume $g \in D^{\prime}\left(z_{0}\right)$, then

a. the integral curve $\{g \exp t X: t \in \mathbb{C}\}$ for $X$ is contained in $D^{\prime}\left(z_{0}\right)$ and;

b. $D^{\prime}\left(z_{0}\right) \cdot g^{-1}=D^{\prime}\left(z_{0}\right) \cdot(g \exp t X)^{-1}$ for all $t \in \mathbb{C}$; i.e., $D^{\prime}\left(g\left(z_{0}\right)\right)=$ $D^{\prime}\left(g \exp t X\left(z_{0}\right)\right)$ for all $t \in \mathbb{C}$.

Moreover, from the above formula and the definition of $\lambda(z)$,

c. $\lambda\left(g\left(z_{0}\right)\right)=\lambda\left(g \exp t X\left(z_{0}\right)\right)$ for all $t \in \mathbb{C}$.

The set $\left\{g \exp t X\left(z_{0}\right): t \in \mathbb{C}\right\}$ is a one-dimensional holomorphic curve in $D$ since $a \neq 0$. Since $-\lambda$ is an exhaustion function for $D$, we have

d. $\left\{g \exp t X\left(z_{0}\right): t \in \mathbb{C}\right\}$ is relatively compact in $D$.

Using the same argument that we used for the last assertion in Corollary 5.1 for $A=\partial D^{\prime}\left(z_{0}\right)$ or $A={\overline{D^{\prime}\left(z_{0}\right)}}^{c}$, we have

$$
\begin{array}{ll}
g \exp t X \in A, & \text { for } t \in \mathbb{C}, \\
A g^{-1}=A(g \exp t X)^{-1}, & \text { for } t \in \mathbb{C},
\end{array}
$$

for $g \in A$.

We conclude that if $-\lambda$ is not strictly plurisubharmonic at $z_{0}$, then for any $g \in D^{\prime}\left(z_{0}\right)$ we obtain a one-dimensional holomorphic curve $g(C) \Subset D$ (where $\left.C:=\left\{\exp t X\left(z_{0}\right): t \in \mathbb{C}\right\}\right)$ on which $\lambda$ is constant, with value $\lambda\left(g\left(z_{0}\right)\right)$. Now take any point $z^{\prime} \in D$. Using 1 . in Proposition 6.1, there exists $g^{\prime} \in D^{\prime}\left(z_{0}\right)$ such that $g^{\prime}\left(z_{0}\right)=z^{\prime}$. Then, as mentioned above, the one-dimensional curve $C^{\prime}:=g^{\prime}(C)$ passes through $z^{\prime}$ and is relatively compact in $D$. Thus if $-\lambda$ is not strictly plurisubharmonic at some point $z_{0} \in D$, then for each point $z^{\prime} \in D$, there exists a one-dimensional holomorphic curve $C^{\prime} \Subset D$ passing through $z^{\prime}$. (We mention that as a curve in $D, C^{\prime}$ is conformally equivalent to $\mathbb{C}, \mathbb{C}^{*}:=\mathbb{C} \backslash\{0\}, \mathbb{P}^{1}$ or a torus.) Hence any exhaustion function $s(z)$ which is plurisubharmonic on $D$, in particular, the function $-\lambda(z)$, must be constant on $C^{\prime}$, and hence the complex Hessian $\left[\frac{\partial^{2} s}{\partial z_{j} \partial \bar{z}_{k}}\left(z^{\prime}\right)\right]$ has a zero eigenvalue.

In this vein, we mention a result of Michel [12] for compact $M$ : if $D$ admits a strictly pseudoconvex boundary point, then $D$ is Stein. This follows in the case of a domain $D$ with $C^{\infty}$ boundary from our results. For if $p \in \partial D$ is a strictly pseudoconvex boundary point and if $-\lambda$ is not strictly plurisubharmonic on $D$, then $\left[\frac{\partial^{2}(-\lambda)}{\partial z_{j} \partial \bar{z}_{k}}\left(z_{0}\right)\right]$ has a zero eigenvalue at some $z_{0} \in D$; 
i.e., $\left.\left[\frac{\partial^{2} \lambda\left(z_{0}+a t\right)}{\partial t \partial \bar{t}}\right]\right|_{t=0}=0$ for some direction $a \in \mathbb{C}^{n}(a \neq 0)$. This implies (a. above) that there exists a nonvanishing left-invariant holomorphic vector field $X$ on $G$ such that if $g \in \partial D^{\prime}\left(z_{0}\right)$, then $g \exp t X \in \partial D^{\prime}\left(z_{0}\right)$ for all $t \in \mathbb{C}$. Take $g^{\prime} \in \partial D^{\prime}\left(z_{0}\right)$ with $g^{\prime}\left(z_{0}\right)=p$; such a $g^{\prime}$ exists by homogeneity. Since $a \neq 0$, the projection of this integral curve $\{g \exp t X: t \in \mathbb{C}\}$ under $\psi_{z_{0}}$ is a one-dimensional holomorphic curve through $p$ lying in $\partial D$, contradicting the strict pseudoconvexity of $\partial D$ at $p$.

We also mention that Diederich-Ohsawa [4] show that if $D$ is a pseudoconvex domain with $C^{\omega}$ boundary in a complex 2-dimensional manifold and $D$ admits a strictly pseudoconvex boundary point, then $D$ is Stein.

What are some sufficient conditions on the pair $(M, G)$ which insure that for any relatively compact, smoothly bounded pseudoconvex domain $D$ in $M$, the $c$-Robin function $\lambda(z)$ for $(D(z), e)$ has the property that $-\lambda(z)$ is strictly plurisubharmonic on $D$ ? We discuss two such conditions. Recall that $H_{z_{0}}^{\prime}$ denotes the connected component of the isotropy subroup $H_{z_{0}}$ of $G$ for $z_{0}$ which contains the identity $e$. First, we say that the pair $(M, G)$ satisfies the three point property if for any triple of distinct points $z_{0}, z_{1}$ and $z_{2}$ in $M$, there exists an element $g \in H_{z_{0}}^{\prime}$ with $g\left(z_{1}\right)=z_{2}$. As an example, take $M=\mathbb{C}$ and $G=\operatorname{Aut} M$, or for, $n>1, M=\mathbb{C}^{n}$ and $G$ the subgroup of Aut $M$ generated by $G L(n, \mathbb{C})$ and translations. Another example is $M=$ $\mathbb{P}^{n}$ and $G=G L(n+1, \mathbb{C})$. However the complex Grassmannian manifold $M=G(k, n)$ with $G=G L(n, \mathbb{C})$ does not satisfy the three point condition if $2 \leq k \leq n-2$ (see 2. in Appendix A). Next, we say that the pair $(M, G)$ satisfies the spanning property if for any $z_{0} \in M$ and for any one-dimensional complex curve $C$ in a neighborhood of $z_{0}$ in $M$ passing through $z_{0}$, we can find $h_{i}, i=1, \ldots, n$, in $H_{z_{0}}^{\prime}$ with the following property: let $C_{i}:=h_{i}(C)$ be the image of $C$ under $h_{i}$; this is a curve in $M$ passing through $z_{0}$; if $a_{i}$ denotes the complex tangent vector of $C_{i}$ at $z_{0}$, then we require that the $n$ vectors $\left\{a_{1}, \ldots, a_{n}\right\}$ span $\mathbb{C}^{n}$. The Grassmannian manifolds $M=G(k, n)$ with $G=G L(n, \mathbb{C})$ satisfy the spanning condition for any $k=1, \ldots, n$; this is $\mathbf{3}$. in Appendix A.

The importance of these notions occurs in the following result.

Theorem 6.2. Let $(M, G)$ satisfy the three point property or the spanning property. Then for any pseudoconvex domain $D \Subset M$ with $C^{\infty}$ boundary, if we let $\lambda(z)$ be the $c$-Robin function for $D$, then $-\lambda(z)$ is a strictly plurisubharmonic exhaustion function for $D$; i.e., $D$ is Stein.

Proof. By Theorem 6.1, $-\lambda(z)$ is a plurisubharmonic exhaustion function for $D$. We prove the strict plurisubharmonicity by contradiction. Suppose that $-\lambda(z)$ is not strictly plurisubharmonic at $z_{0} \in D$. Then the complex 
Hessian $\left[\frac{\partial^{2}(-\lambda)}{\partial z_{j} \partial \bar{z}_{k}}\left(z_{0}\right)\right]$ has a zero eigenvalue. From the above discussion, we conclude that there exists a left-invariant holomorphic vector field $X$ on $G$ such that setting $C:=\left\{\exp t X\left(z_{0}\right): t \in \mathbb{C}\right\}$, the tangent vector of $C$ at $t=0$ is not zero, and for any $g \in D^{\prime}\left(z_{0}\right)$ we obtain a one-dimensional holomorphic curve $g(C):=\left\{g \exp t X\left(z_{0}\right): t \in \mathbb{C}\right\} \Subset D$ on which $\lambda$ is constant with value $\lambda\left(g\left(z_{0}\right)\right)$, and $D^{\prime}\left(g \exp t X\left(z_{0}\right)\right)=D^{\prime}\left(g\left(z_{0}\right)\right)$.

We first treat the case when $(M, G)$ satisfies the three point property. Fix $z_{1} \in C \backslash\left\{z_{0}\right\}$. Given $z \in D$ with $z \neq z_{0}, z_{1}$, from the three-point property we can find $g \in H_{z_{0}}^{\prime}$ such that $g\left(z_{1}\right)=z$. Since $g \in H_{z_{0}}^{\prime} \subset D^{\prime}\left(z_{0}\right)$, it follows that $\lambda$ is constant on the curve $g(C)$ with value $\lambda\left(g\left(z_{0}\right)\right)=\lambda\left(z_{0}\right)$. From $z=g\left(z_{1}\right) \in g(C)$, we have, in particular, $\lambda(z)=\lambda\left(z_{0}\right)$, i.e., $\lambda \equiv$ const. on $D$, a contradiction.

We next treat the case when $(M, G)$ satisfies the spanning condition. Then we can find $h_{i}, i=1, \ldots, n$ in $H_{z_{0}}^{\prime} \subset D^{\prime}\left(z_{0}\right)$ such that $\left\{a_{1}, \ldots, a_{n}\right\}$ span $\mathbb{C}^{n}$, where $a_{i}$ is the complex tangent vector of the curve $h_{i}(C):=$ $h_{i} \exp t X\left(z_{0}\right), t \in \mathbb{C}$ at $z_{0}$. It follows from c. that

$$
\lambda\left(h_{i} \exp t X\left(z_{0}\right)\right)=\lambda\left(h_{i}\left(z_{0}\right)\right)=\lambda\left(z_{0}\right), \quad \text { for all } t \in \mathbb{C} .
$$

In other words, $\lambda$ assumes the same value $\lambda\left(z_{0}\right)$ on each curve $h_{i}(C), i=$ $1, \ldots, n$ with different tangent directions at $z_{0}$ which span $\mathbb{C}^{n}$. Therefore all eigenvalues of the complex Hessian $\left[\frac{\partial^{2}(-\lambda)}{\partial z_{j} \partial \bar{z}_{k}}\left(z_{0}\right)\right]$ are 0 . By the positive semidefiniteness of this matrix, we conclude that $\left[\frac{\partial^{2}(-\lambda)}{\partial z_{j} \partial \bar{z}_{k}}\left(z_{0}\right)\right]$ is the zero matrix.

Now take $z^{\prime} \in D$ with $z^{\prime} \neq z_{0}$. By 1 . in Proposition 6.1 we can find $g^{\prime} \in D^{\prime}\left(z_{0}\right)$ such that $g^{\prime}\left(z_{0}\right)=z^{\prime}$. Let $C^{\prime}=g^{\prime}(C)$. Then $\lambda(z)$ is constant on $C^{\prime}$ by property c., so that $\left[\frac{\partial^{2}(-\lambda)}{\partial z_{j} \partial \bar{z}_{k}}\left(z^{\prime}\right)\right]$ has a zero eigenvalue. Then we can find $h_{i}^{\prime} \in H_{z_{0}}^{\prime}, i=1, \ldots, n$ such that $\left\{b_{1}, \ldots, b_{n}\right\}$ span $\mathbb{C}^{n}$, where $b_{i}$ is the complex tangent vector of the curve $h_{i}^{\prime}\left(C^{\prime}\right)$ at $z^{\prime}$. By the previous argument, all eigenvalues of the complex Hessian $\left[\frac{\partial^{2}(-\lambda)}{\partial z_{j} \partial \bar{z}_{k}}\left(z^{\prime}\right)\right]$ are 0 ; hence this matrix is the zero matrix. Since this argument is valid for any $z^{\prime} \in D$, we conclude that $-\lambda$ is pluriharmonic on $D$. Since $-\lambda$ is an exhaustion function on $D$, $-\lambda$ is constant on $D$ by the minimum principle. This is a contradiction.

As mentioned, the complex Grassmannian manifold $G(k, n)$ satisfies the spanning condition. Therefore, the $c$-Robin function $-\lambda(z)$ for $D$ is a strictly plurisubharmonic exhaustion function for any pseudoconvex domain $D$ with $C^{\infty}$ boundary in $G(k, n)$. This result has been obtained by T. Ueda [19] (also see A. Hirschowitz [3]) by a different method in the more general situation when $D$ is any finite or infinitely sheeted unramified pseudoconvex domain over $G(k, n)$. However, as will be shown in 4. of Appendix A, the flag space $M=\mathcal{F}_{n}$ with $G=G L(n, \mathbb{C})$ for $n \geq 3$ does not satisfy the spanning property. 
To analyze the pseudoconvex domains in $\mathcal{F}_{n}$ with smooth boundary which are not Stein, we shall establish Theorem 6.3 which addresses this issue for general homogeneous spaces.

We use the same notation $M, G, e$ with $\operatorname{dim} G=m$ and $\operatorname{dim} M=n \leq m$. Fix $z \in M$ and let $H_{z}$ be the isotropy subgroup of $G$ for $z$. We let $\psi_{z}$ denote the projection from $G$ onto $M \approx G / H_{z}$. We write $H_{z}^{\prime}$ for the connected component of $H_{z}$ containing $e$. Then $H_{z}^{\prime}$ is a connected, closed, normal Lie subgroup of $H_{z}$ with $\operatorname{dim} H_{z}^{\prime}=m_{0}:=m-n$. Let $\mathfrak{X}$ denote the (complex) Lie algebra consisting of all left-invariant holomorphic vector fields $X$ on $G$. Finally, $\mathfrak{h}_{z}$ denotes the Lie subalgebra of $\mathfrak{X}$ corresponding to the Lie subgroup $H_{z}^{\prime}$ of $G$ with $\operatorname{dim} \mathfrak{h}_{z}=m_{0}$; thus

$$
H_{z}^{\prime}=\left\{\prod_{j=1}^{\nu} \exp t_{j} X_{j}: \nu \in \boldsymbol{Z}^{+}, t_{j} \in \mathbb{C}, X_{j} \in \mathfrak{h}_{z}\right\} .
$$

Let $D \Subset M$ be a pseudoconvex domain with smooth boundary which is not Stein. We form the $c$-Robin function $\lambda(z)$ for $D$. Recall that we defined, for a given $z \in D$,

$$
D(z):=\{g \in G \mid g(z) \in D\}=\psi_{z}^{-1}(D) \subset G,
$$

and considered the variation $\mathcal{D}$ of domains $D(z)$ in $G$ with parameter $z \in D$ :

$$
\mathcal{D}: z \in D \rightarrow D(z) \subset G .
$$

The $c$-Robin function $\lambda(z)$ was defined as the $c$-Robin constant for $(D(z), e)$; precisely, for the connected component $D^{\prime}(z)$ of $D(z)$ containing $e$. Then $-\lambda(z)$ is a plurisubharmonic exhaustion function for $D$. Under the assumption that $D$ is not Stein, there exists a point $z_{0} \in D$ such that $-\lambda(z)$ is not strictly plurisubharmonic at $z_{0}$, i.e., there exists $a \in \mathbb{C}^{n}(a \neq 0)$ such that $\left.\left[\frac{\partial^{2} \lambda\left(z_{0}+a t\right)}{\partial t \partial \bar{t}}\right]\right|_{t=0}=0$. If we take an arbitrary vector field $X \in \mathfrak{X}$ such that $\left.\left[\frac{d \exp t X\left(z_{0}\right)}{d t}\right]\right|_{t=0}=a$, then we have:

$$
\begin{aligned}
\text { o. } & {\left.\left[\frac{\partial^{2} \lambda\left(\exp t X\left(z_{0}\right)\right)}{\partial t \partial \bar{t}}\right]\right|_{t=0}=0 ; } \\
\text { i. } & \left\{\exp t X\left(z_{0}\right): t \in \mathbb{C}\right\} \subset D \text { and } \lambda\left(\exp t X\left(z_{0}\right)\right)=\lambda\left(z_{0}\right), t \in \mathbb{C} ; \text { indeed } \\
\text { ii. } & \left\{\exp t X\left(z_{0}\right): t \in \mathbb{C}\right\} \Subset D ; \text { moreover } \\
\text { iii. } & \{\exp t X: t \in \mathbb{C}\} \subset D^{\prime}\left(z_{0}\right) \text { and } D^{\prime}\left(\exp t X\left(z_{0}\right)\right)=D^{\prime}\left(z_{0}\right), t \in \mathbb{C} .
\end{aligned}
$$

From properties of the Robin function $\lambda(z)$, these four conditions are equivalent. We write $o$. $\sim$ iii. and we consider

$$
\mathfrak{X}_{z_{0}}:=\{X \in \mathfrak{X}: X \text { satisfies one (all) conditions(s) o. } \sim \text { iii. }\} \text {. }
$$


We note that

$$
\mathfrak{h}_{z_{0}} \subsetneq \mathfrak{X}_{z_{0}} \subsetneq \mathfrak{X}
$$

For suppose $X \in \mathfrak{h}_{z_{0}}$. Then $\exp t X \in H_{z_{0}}^{\prime}$ for $t \in \mathbb{C}$. Hence $\exp t X\left(z_{0}\right)=z_{0}$, so that $X \in \mathfrak{X}_{z_{0}}$. Under the assumption that $D$ is not Stein, we have already shown that $\operatorname{dim} \mathfrak{X}_{z_{0}}>\operatorname{dim} \mathfrak{h}_{z_{0}}=m_{0}$. Next, since $-\lambda$ is a plurisubharmonic exhaustion function for $D,-\lambda$ is not pluriharmonic. Following the argument at the end of the proof of Theorem 6.2, we see that $\operatorname{dim} \mathfrak{X}>\operatorname{dim} \mathfrak{X}_{z_{0}}$.

We want to prove that $\mathfrak{X}_{z_{0}}$ is a complex Lie subalgebra of $\mathfrak{X}$, and verify some properties of $\mathfrak{X}_{z_{0}}$. To this end, we begin with a technical lemma.

Lemma 6.3. Let $\nu \in \boldsymbol{Z}^{+}$and let $g_{i}, h_{i} \in H_{z_{0}}^{\prime} ; X_{i} \in \mathfrak{X}_{z_{0}} ; t_{i} \in \mathbb{C}, i=$ $1,2, \ldots, \nu$ and $g \in D^{\prime}\left(z_{0}\right)$. Then

$$
\begin{aligned}
& \text { (1) } g\left[\prod_{i=1}^{\nu} g_{i}\left(\exp t_{i} X_{i}\right) h_{i}^{-1}\right] \in D^{\prime}\left(z_{0}\right) \\
& \text { (2) } \lambda\left(g\left[\prod_{i=1}^{\nu}\left(g_{i}\left(\exp t_{i} X_{i}\right) h_{i}^{-1}\right]\left(z_{0}\right)\right)=\lambda\left(g\left(z_{0}\right)\right) .\right.
\end{aligned}
$$

Proof. By the definition of the $c$-Robin constant $\lambda(z)$ for $(D(z), e)$, to verify (2) it suffices to prove

$$
\left(2^{\prime}\right) \quad D^{\prime}\left(g\left[\prod_{i=1}^{\nu} g_{i}\left(\exp t_{i} X_{i}\right) h_{i}^{-1}\right]\left(z_{0}\right)\right)=D^{\prime}\left(g\left(z_{0}\right)\right) .
$$

We prove (1) and $\left(2^{\prime}\right)$ by induction. We will utilize some of the properties $a$. $-d$. mentioned after the proof of Theorem 6.1. Let $\nu=1$. By 2. in Proposition 6.1, we have $g g_{1} \in D^{\prime}\left(z_{0}\right)$. This together with property a. implies $C:=\left\{g g_{1} \exp t X_{1} \in G: t \in \mathbb{C}\right\} \subset D^{\prime}\left(z_{0}\right)$. We see from property III. of Proposition 6.2 and $h_{1}^{-1} \in H_{z_{0}}^{\prime}$ that $C h_{1}^{-1} \subset D^{\prime}\left(z_{0}\right) h_{1}^{-1}=D^{\prime}\left(h_{1}\left(z_{0}\right)\right)=$ $D^{\prime}\left(z_{0}\right)$, so that (1) for $\nu=1$ is proved. Furthermore, since $g g_{1} \in D^{\prime}\left(z_{0}\right)$ and $h_{1}^{-1}\left(z_{0}\right)=z_{0}$, it follows from property $\mathrm{b}$. that

$D^{\prime}\left(g g_{1}\left(\exp t_{1} X_{1}\right) h^{-1}\left(z_{0}\right)\right)=D^{\prime}\left(g g_{1}\left(\exp t_{1} X_{1}\right)\left(z_{0}\right)\right)=D^{\prime}\left(g g_{1}\left(z_{0}\right)\right)=D^{\prime}\left(g\left(z_{0}\right)\right)$,

which proves $\left(2^{\prime}\right)$ for $\nu=1$. Next we assume that $(1)$ and $\left(2^{\prime}\right)$ are true for $\nu \leq k$ and we shall prove them for $\nu=k+1$. We define, for $t \in \mathbb{C}$,

$$
k(t):=g\left[\prod_{i=1}^{k} g_{i}\left(\exp t_{i} X_{i}\right) h_{i}^{-1}\right]\left(g_{k+1}\left(\exp t X_{k+1}\right) h_{k+1}^{-1}\right) \in G,
$$

and we consider the curve $C:=\{k(t) \in G: t \in \mathbb{C}\}$ in $G$. It suffices to prove that $C \subset D^{\prime}\left(z_{0}\right)$ and $D^{\prime}\left(k(t)\left(z_{0}\right)\right)=D^{\prime}\left(g\left(z_{0}\right)\right), t \in \mathbb{C}$. Note that

$$
k(t)=k_{0} \cdot\left(\exp t X_{k+1}\right) h_{k+1}^{-1},
$$

where

$$
k_{0}:=g\left[\prod_{i=1}^{k-1} g_{i}\left(\exp t_{i} X_{i}\right) h_{i}^{-1}\right]\left(g_{k}\left(\exp t_{k} X_{k}\right)\left(g_{k+1}^{-1} h_{k}\right)^{-1}\right) .
$$


Then the induction hypothesis for (1) in the case $\nu=k$ implies that $k_{0} \in$ $D^{\prime}\left(z_{0}\right)$ since $g_{k+1}^{-1} h_{k} \in H_{z_{0}}^{\prime}$; thus property a. yields that $k_{0} \exp t X_{k+1} \in$ $D^{\prime}\left(z_{0}\right), t \in \mathbb{C}$. Since $h_{k+1}^{-1} \in H_{z_{0}}^{\prime}$, it follows from 2. in Proposition 6.1 that $C \subset D^{\prime}\left(z_{0}\right)$, so that (1) for the case $\nu=k+1$ is valid. Moreover, since $g \prod_{i=1}^{k} g_{i}\left(\exp t_{i} X_{i}\right) h_{i}^{-1} \in D^{\prime}\left(z_{0}\right)$ (by the induction hypothesis (1) in the case $\nu=k$ ) and $g_{k+1} \exp t X_{k+1} h_{k+1}^{-1} \in D^{\prime}\left(z_{0}\right)$ (which follows by (1) in the case $\nu=1$ for $g=e$ ), we again utilize property III of Proposition 6.2 (twice) to obtain

$$
\begin{aligned}
D^{\prime}\left(k(t)\left(z_{0}\right)\right) & =D^{\prime}\left(\left[g \prod_{i=1}^{k} g_{i}\left(\exp t X_{i}\right) h_{i}^{-1}\right]\left(g_{k+1} \exp t X_{k+1} h_{k+1}^{-1}\right)\left(z_{0}\right)\right) \\
& =D^{\prime}\left(\left(g_{k+1} \exp t X_{k+1} h_{k+1}^{-1}\right)\left(z_{0}\right)\right)\left[g \prod_{i=1}^{k} g_{i}\left(\exp t X_{i}\right) h_{i}^{-1}\right]^{-1} \\
& =D^{\prime}\left(z_{0}\right)\left[g \prod_{i=1}^{k} g_{i}\left(\exp t X_{i}\right) h_{i}^{-1}\right]^{-1} \\
& =D^{\prime}\left(\left[g \prod_{i=1}^{k} g_{i}\left(\exp t X_{i}\right) h_{i}^{-1}\right]\left(z_{0}\right)\right) \\
& =D^{\prime}\left(g\left(z_{0}\right)\right) .
\end{aligned}
$$

The last equality follows from $\left(2^{\prime}\right)$ in the case $\nu=k$. This proves $\left(2^{\prime}\right)$ for $\nu=k+1$.

Arguing as in (1) but using (6.10) and (6.9) for $A=\partial D^{\prime}\left(z_{0}\right),{\overline{D^{\prime}\left(z_{0}\right)}}^{c}$ instead of a. and III. in Proposition (6.2) for $D^{\prime}\left(z_{0}\right)$, we obtain

$$
g\left[\prod_{i=1}^{\nu} g_{i}\left(\exp t_{i} X_{i}\right) h_{i}^{-1}\right] \in A, \quad g \in A .
$$

We can now prove the following fundamental result.

\section{Lemma 6.4.}

1. $\mathfrak{X}_{z_{0}}$ is a complex Lie subalgebra of $\mathfrak{X}$.

2. Let $X \in \mathfrak{X}_{z_{0}}$ and $g \in H_{z_{0}}^{\prime}$. Then any $Y \in \mathfrak{X}$ which satisfies

$$
\left.\left[\frac{d \exp t Y\left(z_{0}\right)}{d t}\right]\right|_{t=0}=\left.\left[\frac{d g \exp t X\left(z_{0}\right)}{d t}\right]\right|_{t=0}
$$

belongs to $\mathfrak{X}_{z_{0}}$.

3. For $z_{1} \in D$ we have $\operatorname{dim} \mathfrak{X}_{z_{1}}=\operatorname{dim} \mathfrak{X}_{z_{0}}$.

Note that (6.14) says that the tangent vector of the curve $C_{Y}:=\left\{\exp t Y\left(z_{0}\right) \in\right.$ $M: t \in \mathbb{C}\}$ at $z_{0}$ in $M$ coincides with that of the curve $g\left(C_{X}\right)$ at $z_{0}$.

Proof. To verify 1 ., let $X, Y \in \mathfrak{X}_{0}$. Fix $\alpha, \beta \in \mathbb{C}$. If we set $t_{1}=\alpha t, t_{2}=$ $\beta t ; X_{1}=X, X_{2}=Y$; and $g_{1}=g_{2}=h_{1}=h_{2}=e$ in Lemma 6.3 (2), we obtain

$$
\lambda\left((\exp \alpha t X)(\exp \beta t Y)\left(z_{0}\right)\right)=\lambda\left(z_{0}\right), \quad \text { for } t \in \mathbb{C} .
$$


Then as we deduced formula (5.7) from (5.6), we obtain

$$
\left.\left[\frac{\partial^{2} \lambda\left(\exp (\alpha t X+\beta t Y)\left(z_{0}\right)\right)}{\partial t \partial \bar{t}}\right]\right|_{t=0}=\left.\left[\frac{\partial^{2} \lambda\left(z_{0}\right)}{\partial t \partial \bar{t}}\right]\right|_{t=0}=0 .
$$

Hence $\alpha X+\beta Y \in \mathfrak{X}_{z_{0}}$. Next, if we set $t_{4}=t_{3}=-\sqrt{t}, t_{2}=t_{1}=\sqrt{t} ; X_{4}=$ $X_{2}=Y, X_{3}=X_{1}=X ; g_{i}=h_{i}=e(i=1, \ldots, 4)$ in Lemma 6.3 (2), we obtain

$\lambda\left(\left[(\exp (-\sqrt{t}) Y)(\exp (-\sqrt{t}) X)(\exp \sqrt{t} Y)(\exp \sqrt{t} X)\left(z_{0}\right)\right]\right)=\lambda\left(z_{0}\right), \quad$ for $t \in \mathbb{C}$.

Then as we deduced (5.9) from (5.8), we obtain

$$
\left.\left[\frac{\partial^{2} \lambda\left((\exp t[X, Y])\left(z_{0}\right)\right)}{\partial t \partial \bar{t}}\right]\right|_{t=0}=\left.\left[\frac{\partial^{2} \lambda\left(z_{0}\right)}{\partial t \partial \bar{t}}\right]\right|_{t=0}=0
$$

This shows that $[X, Y] \in \mathfrak{X}_{z_{0}}$, and 1 . is proved.

To prove 2., we let $X \in \mathfrak{X}_{z_{0}}, g \in H_{z_{0}}^{\prime}$ and assume $Y \in \mathfrak{X}$ satisfies (6.14). By (2) of Lemma 6.3, for $g \in H_{z_{0}}^{\prime}$ we have

$$
\lambda\left(g \exp t X\left(z_{0}\right)\right)=\lambda\left(g\left(z_{0}\right)\right)=\lambda\left(z_{0}\right), \quad \text { for } \in \mathbb{C},
$$

so that $\left.\left[\frac{\partial^{2} \lambda\left(g \exp t X\left(z_{0}\right)\right)}{\partial t \partial \bar{t}}\right]\right|_{t=0}=0$. It follows from condition (6.14) and Proposition 5.1 that

$$
\left.\left[\frac{\partial^{2} \lambda\left(\exp t Y\left(z_{0}\right)\right)}{\partial t \partial \bar{t}}\right]\right|_{t=0}=0
$$

and hence $Y \in \mathfrak{X}_{z_{0}}$.

Assertion 3. is intuitively clear from the property that, for $g \in D^{\prime}\left(z_{0}\right)$,

$$
\lambda\left(g\left(z_{0}\right)\right)=\lambda\left(g \exp t X\left(z_{0}\right)\right)
$$

for all $t \in \mathbb{C}$ (this is c. listed after the proof of Theorem 6.1). The rigorous proof is fairly technical. We begin by setting $\operatorname{dim} \mathfrak{X}_{z_{0}}=m_{0}+m_{1}$ and $m_{2}=$ $m-\left(m_{0}+m_{1}\right)$ where $m=\operatorname{dim} G$ and $m_{0}=\operatorname{dim} \mathfrak{h}_{z_{0}}$. Note that $m_{0}$ is independent of the point $z_{0}$ but, apriori, $m_{1}$ may depend on $z_{0}$. We consider the following direct sum decompositions:

$$
\mathfrak{X}_{z_{0}}=\mathfrak{h}_{z_{0}}+\mathfrak{k}_{z_{0}} ; \quad \mathfrak{X}=\mathfrak{h}_{z_{0}} \dot{+} \mathfrak{k}_{z_{0}} \dot{+} \mathfrak{l}_{z_{0}} .
$$

Here $\mathfrak{k}_{z_{0}}$ and $\mathfrak{l}_{z_{0}}$ are defined by these relations. Let

$$
\left\{X_{1}, \ldots, X_{m_{2}}\right\},\left\{Y_{1}, \ldots, Y_{m_{1}}\right\} \text { and }\left\{Z_{1}, \ldots, Z_{m_{0}}\right\}
$$


be bases of $\mathfrak{l}_{z_{0}}, \mathfrak{k}_{z_{0}}$ and $\mathfrak{h}_{z_{0}}$. We fix canonical local coordinates on $V \subset \mathbb{C}^{m}$ for a neighborhood $\mathcal{V} \subset G$ of $e$ such that $g \in \mathcal{V}$ corresponds to $(\xi ; \eta ; \zeta) \in V$; precisely,

$$
\begin{aligned}
g & =\prod_{i=1}^{m_{2}} \exp a_{i} X_{i} \prod_{j=1}^{m_{1}} \exp b_{j} Y_{j} \prod_{k=1}^{m_{0}} \exp c_{k} Z_{k} \in \mathcal{V} \\
& \longleftrightarrow(\xi ; \eta ; \zeta)=\left(a_{1}, \ldots, a_{m_{2}} ; b_{1}, \ldots, b_{m_{1}} ; c_{1}, \ldots, c_{m_{0}}\right) \in V,
\end{aligned}
$$

where $\left|a_{i}\right|,\left|b_{j}\right|,\left|c_{k}\right|<\rho$ (for $\rho>0$ sufficiently small), $e$ corresponds to $0=$ $(0 ; 0 ; 0)$, and

$$
\psi_{z_{0}}(g) \longleftrightarrow(\xi ; \eta)=\left(a_{1}, \ldots, a_{m_{2}} ; b_{1}, \ldots, b_{m_{1}}\right) \in V^{\prime} \subset \mathbb{C}^{m_{2}+m_{1}}
$$

are local coordinates of the neighborhood $\mathcal{V}^{\prime}=\psi_{z_{0}}(\mathcal{V}) \subset M$ of $z_{0}$ where $z_{0}$ corresponds to $0=(0 ; 0)$.

Fix $z_{1} \in D$. Using 1. in Proposition 6.1 we can find $g_{1} \in D^{\prime}\left(z_{0}\right)$ with $g_{1}\left(z_{0}\right)=z_{1}$. As in (6.15) we consider the direct sums

$$
\mathfrak{X}_{z_{1}}=\mathfrak{h}_{z_{1}}+\mathfrak{k}_{z_{1}} ; \quad \mathfrak{X}=\mathfrak{h}_{z_{1}}+\mathfrak{k}_{z_{1}}+\mathfrak{l}_{z_{1}} \text {. }
$$

We set $\operatorname{dim} \mathfrak{X}_{z_{1}}=m_{0}+m_{1}^{\prime}$ where $m_{0}=\operatorname{dim} \mathfrak{h}_{z_{1}}$. To verify 3 . it suffices to show that $m_{1}=m_{1}^{\prime}$. Similar to before, we consider bases

$$
\left\{X_{1}^{\prime}, \ldots, X_{m_{2}^{\prime}}^{\prime}\right\},\left\{Y_{1}^{\prime}, \ldots, Y_{m_{1}^{\prime}}^{\prime}\right\} \text { and }\left\{Z_{1}^{\prime}, \ldots, Z_{m_{0}}^{\prime}\right\}
$$

of $\mathfrak{l}_{z_{1}}, \mathfrak{k}_{z_{1}}$ and $\mathfrak{h}_{z_{1}}$. Here, $m_{2}^{\prime}=m-\left(m_{0}+m_{1}^{\prime}\right)$. We fix canonical local coordinates on $V_{1} \subset \mathbb{C}^{m}$ for a neighborhood $\mathcal{V}_{1} \subset G$ of $e$ with

$$
\begin{aligned}
g & =\prod_{i=1}^{m_{2}^{\prime}} \exp a_{i} X_{i}^{\prime} \prod_{j=1}^{m_{1}^{\prime}} \exp b_{i} Y_{j}^{\prime} \prod_{k=1}^{m_{0}} \exp c_{k} Z_{k}^{\prime} \in \mathcal{V}_{1} \\
& \longleftrightarrow\left(\xi^{\prime} ; \eta^{\prime} ; \zeta^{\prime}\right)=\left(a_{1}, \ldots, a_{m_{2}^{\prime}} ; b_{1}, \ldots, b_{m_{1}^{\prime}} ; c_{1}, \ldots, c_{m_{0}}\right) \in V_{1},
\end{aligned}
$$

where $\left|a_{i}\right|,\left|b_{j}\right|,\left|c_{k}\right|<\rho$ for $\rho>0$ sufficiently small; $e$ corresponds to $0=$ $(0 ; 0 ; 0)$; and

$$
\psi_{z_{1}}(g) \longleftrightarrow\left(\xi^{\prime} ; \eta^{\prime}\right)=\left(a_{1}, \ldots, a_{m_{2}^{\prime}} ; b_{1}, \ldots, b_{m_{1}^{\prime}}\right) \in V_{1}^{\prime} \subset \mathbb{C}^{m_{2}^{\prime}+m_{1}^{\prime}}
$$

are local coordinates for the neighborhood $\mathcal{V}_{1}^{\prime}=\psi_{z_{1}}(\mathcal{V}) \subset M$ of $z_{1}$ where $z_{1}$ corresponds to $0=(0 ; 0)$.

Given $X+Y=\sum_{i=1}^{m_{2}} a_{i} X_{i}+\sum_{j=1}^{m_{1}} b_{j} Y_{j} \in \mathfrak{l}_{z_{0}}+\mathfrak{k}_{z_{0}}$, we consider the curve $C:=\left\{\exp t(X+Y)\left(z_{0}\right): t \in \mathbb{C}\right\}$ in $M$ with initial value $z_{0}$ and tangent vector $\tau:=\left.\left[\frac{d \exp t(X+Y)\left(z_{0}\right)}{d t}\right]\right|_{t=0} \in \mathbb{C}^{m_{2}+m_{1}}=\mathbb{C}^{m-m_{0}}$ at $z_{0}$ in $M$. Since

$$
\exp \left[t(X+Y)+O\left(t^{2}\right)\right]=\prod_{i=1}^{m_{2}} \exp t a_{i} X_{i} \prod_{j=1}^{m_{1}} \exp t b_{j} Y_{j}
$$


near $t=0$, we can write $\tau=\left(a_{1}, \ldots, a_{m_{2}} ; b_{1}, \ldots, b_{m_{1}}\right):=(\mathbf{a}, \mathbf{b})$ in terms of the canonical local coordinates of $V^{\prime}$. We note that this correspondence $X+Y \in \mathfrak{k}_{z_{0}}+\mathfrak{l}_{z_{0}} \longleftrightarrow \tau=(\mathbf{a} ; \mathbf{b}) \in \mathbb{C}^{m_{2}+m_{1}}$ is linear and one-to-one; moreover, $X+0(0+Y)$ corresponds to $(\mathbf{a} ; 0)((0 ; \mathbf{b}))$. We consider the image curve $g_{1}(C)$ with initial value at $z_{1}$ in $M$ and tangent vector $\tau^{\prime}:=\left.\left[\frac{d g_{1} \exp t(X+Y)\left(z_{0}\right)}{\partial t}\right]\right|_{t=0} \in$ $\mathbb{C}^{m_{2}^{\prime}+m_{1}^{\prime}}=\mathbb{C}^{m-m_{0}}$ at $z_{1}$ in $M$ (in terms of the local coordinates $\left(\xi^{\prime} ; \eta^{\prime}\right)$ for $V_{1}^{\prime}$ ). Then the correspondence $L: \tau \in \mathbb{C}^{m-m_{0}} \rightarrow \tau^{\prime} \in \mathbb{C}^{m-m_{0}}$ is linear and one-toone since $\tau \neq 0$ implies $\tau_{1} \neq 0$. We consider the case when $X=0$, that is, $\mathbf{a}=0$, and $Y=\sum_{j=1}^{m_{1}} b_{j} Y_{j} \neq 0$, so that $\tau=\left(0 ; b_{1}, \ldots, b_{m_{1}}\right) \neq(0 ; 0)$. Since $Y \in \mathfrak{X}_{z_{0}}$ and $g_{1} \in D^{\prime}\left(z_{0}\right)$, it follows from property c. that $\lambda(z)$ is constant on the curve $g_{1}(C)$. Now by a standard result in the theory of homogeneous spaces there exists $\tilde{Y} \in \mathfrak{X}$ such that the tangent vector $\left.\left[\frac{d \exp t \tilde{Y}\left(z_{1}\right)}{d t}\right]\right|_{t=0}$ of the curve $\left\{\exp t \tilde{Y}\left(z_{1}\right): t \in \mathbb{C}\right\}$ at $z_{1}$ in $M$ equals $\tau^{\prime}$ (in the local coordinates $\left(\xi^{\prime} ; \eta^{\prime}\right)$ for $\left.V_{1}^{\prime}\right)$; thus $\left.\left[\frac{\partial^{2} \lambda\left(\exp t \tilde{Y}\left(z_{1}\right)\right)}{\partial t \partial \bar{t}}\right]\right|_{t=0}=0$, so that $\widetilde{Y} \in \mathfrak{X}_{z_{1}}$. We conclude that $\tau^{\prime}$ is of the form $\tau^{\prime}=\left(0 ; b_{1}^{\prime}, \ldots, b_{m_{1}^{\prime}}^{\prime}\right) \in \mathbb{C}^{m_{2}^{\prime}+m_{1}^{\prime}}$. Since $L$ is linear and $\tau^{\prime} \neq(0 ; 0)$, we have $m_{1} \leq m_{1}^{\prime}$. Reversing the roles of $z_{0}$ and $z_{1}$ gives the reverse inequality; hence $m_{1}^{\prime}=m_{1}$.

Using 1. in Lemma 6.4 we can construct the connected Lie subgroup $\Sigma_{z_{0}}$ of $G$ which corresponds to $\mathfrak{X}_{z_{0}}$, and the subset $\sigma_{z_{0}}$ of $M$ :

$$
\begin{aligned}
\Sigma_{z_{0}} & =\left\{\prod_{i=1}^{\nu} \exp t_{i} X_{i} \in G: \nu \in \boldsymbol{Z}^{+}, t_{i} \in \mathbb{C}, X_{i} \in \mathfrak{X}_{z_{0}}\right\} ; \\
\sigma_{z_{0}} & :=\Sigma_{z_{0}}\left(z_{0}\right):=\left\{g\left(z_{0}\right) \in M: g \in \Sigma_{z_{0}}\right\} .
\end{aligned}
$$

We have the following.

\section{Corollary 6.2.}

1. If $g \in D^{\prime}\left(z_{0}\right)$, then $g \Sigma_{z_{0}} \subset D^{\prime}\left(z_{0}\right) ; \lambda(z)=$ const. $=\lambda\left(g\left(z_{0}\right)\right)$ on $g \sigma_{z_{0}}$; and $g \sigma_{z_{0}} \Subset D$.

2. For $z_{1} \in D$, we define $\Sigma_{z_{1}} \subset G$ and $\sigma_{z_{1}}=\Sigma_{z_{1}}\left(z_{1}\right) \subset M$ as in (6.16). Then we have, for $g \in D^{\prime}\left(z_{1}\right), \lambda=$ const. $=\lambda\left(g\left(z_{1}\right)\right)$ on $g \sigma_{z_{1}}$; and $g \sigma_{z_{1}} \Subset D$.

3. If $g \in \partial D^{\prime}\left(z_{0}\right)$, then $g \Sigma_{z_{0}} \subset \partial D^{\prime}\left(z_{0}\right)$ and $g \sigma_{z_{0}} \subset \partial D$.

Proof. The first assertion follows from Lemma 6.3 and (6.16). To verify 2., let $z_{1} \in D$ and take $g \in D^{\prime}\left(z_{1}\right)$ with $z_{1}=g\left(z_{0}\right)$. Using $1 ., z_{0}$ and $z_{1}$ play the same role as in 3. of Lemma 6.4, showing that 2. for $z_{1}$ as well as 2 . for $z_{0}$ are valid. The first formula in 3. follows from (6.13). The second formula follows from the first and the fact that $\left[\partial D^{\prime}\left(z_{0}\right)\right]\left(z_{0}\right)=\partial D$ (cf., (6.8) $)$. 
By 1. in Proposition 6.1 we thus have

$$
\begin{aligned}
D & =\bigcup_{g \in D^{\prime}\left(z_{0}\right)} g \sigma_{z_{0}} \quad \text { with } g \sigma_{z_{0}} \Subset D ; \\
\partial D & =\bigcup_{g \in \partial D^{\prime}\left(z_{0}\right)} g \sigma_{z_{0}} .
\end{aligned}
$$

Note that we have not proved that (6.17) provide foliations of $D$ and $\partial D$. Moreover, we remark that if $g \in{\overline{D^{\prime}\left(z_{0}\right)}}^{c}$, then $g \Sigma_{z_{0}} \subset{\overline{D^{\prime}\left(z_{0}\right)}}^{c}$ (as can be seen using an argument similar to that used to verify the first assertions in 1 . or 3$.$) ; however, it is not necessarily true that g \sigma_{z_{0}} \subset \bar{D}^{c}$. In Theorem 6.4 we will need a fairly technical argument to find a domain $K$ with $D \Subset K \subset M$ such that $g \sigma_{z_{0}} \Subset \bar{D}^{c}$ for $g \in K^{\prime}(z) \cap{\overline{D^{\prime}(z)}}^{c}$.

We introduce the following terminology. Let $\sigma$ be a subset of $M$. We call $\sigma$ a $\mu$-dimensional non-singular, generalized ( $f$-generalized) analytic set in $M$ if, for any point $z \in \sigma(z \in M)$, there exists a neighborhood $V$ of $z$ in $M$ such that each connected component of $V \cap \sigma$ is a $\mu$-dimensional nonsingular analytic set in $V$. Moreover, if $\sigma$ admits no non-constant bounded plurisubharmonic functions, then $\sigma$ is said to be parabolic. As a simple example to illustrate the difference between generalized and $f$-generalized analytic sets, let

$$
\Sigma=\left\{z=\left(z_{1}, z_{2}\right) \in \mathbb{C}^{2}: z_{2}=z_{1}\right\}
$$

and take $\sigma:=\Sigma \cap B$ where $B$ is the open unit ball

$$
B=\left\{z=\left(z_{1}, z_{2}\right) \in \mathbb{C}^{2}:\left|z_{1}\right|^{2}+\left|z_{2}\right|^{2}<1\right\} .
$$

Then $\sigma$ is a generalized analytic set in $\mathbb{C}^{2}$ but it is not an $f$-generalized analytic set in $\mathbb{C}^{2}$ because for $z=\left(z_{1}, z_{1}\right) \in \partial B$, there is no neighborhood $V$ of $z$ in $\mathbb{C}^{2}$ such that $V \cap \sigma$ is an analytic set in $V$.

We first prove the main theorem in case the isotropy subgroup $H_{z}$ is connected.

Theorem 6.3. Let $M$ be a complex homogeneous space of finite dimension $n$ and let $G$ be a connected complex Lie transformation group of finite dimension $m \geq n$ which acts transitively on $M$. Assume that the isotropy subgroup $H_{z}$ of $G$ for $z \in M$ is connected. Let $D$ be a pseudoconvex domain in $M$ with smooth boundary. Assume that $D$ is not Stein and fix $z_{0} \in D$. Then:

1-a. The subset $\sigma_{z_{0}}=\Sigma_{z_{0}}\left(z_{0}\right)$ of $M$ defined in (6.16) is a parabolic irreducible $m_{1}$-dimensional non-singular $f$-generalized analytic set in $M$ passing through $z_{0}$ satisfying the following properties:

(o) $\sigma_{z_{0}} \Subset D ; \Sigma_{z_{0}}=G \cap$ Aut $\sigma_{z_{0}} ; \Sigma_{z_{0}}$ acts transitively on $\sigma_{z_{0}}$; and $\sigma_{z_{0}} \approx \Sigma_{z_{0}} / H_{z_{0}}$. 
(i) $M(D)$ is foliated by cosets $g \sigma_{z_{0}}, g \in G\left(g \in D\left(z_{0}\right)\right)$ and each set $g \sigma_{z_{0}}$ is relatively compact in $M(D)$ :

$$
M=\bigcup_{g \in G} g \sigma_{z_{0}} \quad \text { and } \quad D=\bigcup_{g \in D\left(z_{0}\right)} g \sigma_{z_{0}} .
$$

(ii) Any parabolic non-singular generalized analytic set $\sigma$ in $M$ which is relatively compact in $D$ is contained in a set $g \sigma_{z_{0}}$ for some $g \in D\left(z_{0}\right)$.

1-b. Assume that $\sigma_{z_{0}}$ in 1-a. is closed in $M$. Then

(o) $\sigma_{z_{0}}$ is an irreducible, $m_{1}$-dimensional compact non-singular analytic set in $D$ and $\Sigma_{z_{0}}$ is a closed connected Lie subgroup of $G$.

(i) Let $M_{0}:=G / \Sigma_{z_{0}}$ and let $\pi_{0}: M=G / H_{z_{0}} \mapsto M_{0}$ be the canonical projection. Then $\pi_{0}^{-1}(\zeta) \approx \sigma_{z_{0}}$ (as complex manifolds) for each $\zeta \in M_{0}$; moreover, there exists a Stein domain $D_{0} \Subset M_{0}$ with smooth boundary such that $D=\pi_{0}^{-1}\left(D_{0}\right)$.

Proof. From the hypotheses of the theorem and 2. of Corollary 6.2 the function $-\lambda(z)$ is not strictly plurisubharmonic at $z_{0}$. We maintain the notation $\mathfrak{h}_{z_{0}}$ and $\mathfrak{X}_{z_{0}}$ utilized in the proof of Lemma 6.4. Since $H_{z_{0}}$ is assumed to be connected, i.e., $H_{z_{0}}=H_{z_{0}}^{\prime}$, the Lie subalgebra $\mathfrak{h}_{z_{0}}$ of $\mathfrak{X}$ corresponds to the Lie subgroup $H_{z_{0}}$ in $G ; D\left(z_{0}\right)$ is connected in $G$, i.e., $D\left(z_{0}\right)=D^{\prime}\left(z_{0}\right)$ and $H_{z_{0}} \subset D\left(z_{0}\right)$; moreover, each $D(z) \subset G, z \in D$, is connected. We let $\Sigma_{z_{0}}$ denote the connected Lie subgroup of $G$ corresponding to the Lie subalgebra $\mathfrak{X}_{z_{0}}$. We consider the direct sum decompositions from (6.15):

$$
\mathfrak{X}_{z_{0}}=\mathfrak{h}_{z_{0}} \dot{+} \mathfrak{k}_{z_{0}} ; \quad \mathfrak{X}=\mathfrak{h}_{z_{0}}+\mathfrak{k}_{z_{0}} \dot{+} \mathfrak{l}_{z_{0}} .
$$

Here $\operatorname{dim} \mathfrak{h}_{z_{0}}=m_{0}$ and we set $\operatorname{dim} \mathfrak{X}_{z_{0}}:=m_{0}+m_{1}$ and $\operatorname{dim} \mathfrak{l}_{z_{0}}:=m_{2}$ so that $m=m_{0}+m_{1}+m_{2}$. Let $X=\left\{X_{1}, \ldots, X_{m_{2}}\right\}, Y=\left\{Y_{1}, \ldots, Y_{m_{1}}\right\}$ and $Z=\left\{Z_{1}, \ldots, Z_{m_{0}}\right\}$ be bases of $\mathfrak{l}_{z_{0}}, \mathfrak{k}_{z_{0}}$ and $\mathfrak{h}_{z_{0}}$. Then we have

$$
\begin{aligned}
& H_{z_{0}}=\left\{\prod_{j=1}^{\nu} \exp t_{j} A_{j}: \nu \in Z^{+}, t_{j} \in \mathbb{C}, A_{j} \in Z\right\} \\
& \Sigma_{z_{0}}=\left\{\prod_{j=1}^{\nu} \exp t_{j} A_{j}: \nu \in Z^{+}, t_{j} \in \mathbb{C}, A_{j} \in Y \cup Z\right\} .
\end{aligned}
$$

Note first that $H_{z_{0}}$ is an $m_{0}$-dimensional, non-singular closed analytic set in $G$. From the (holomorphic) Frobenius theorem stated in the beginning of section $5, \Sigma_{z_{0}}$ is an $\left(m_{0}+m_{1}\right)$-dimensional non-singular $f$-generalized anaytic set in $G$ with the property that $G$ is foliated by the sets $\left\{g H_{z_{0}}\right\}_{g \in G}$ as well as by the sets $\left\{g \Sigma_{z_{0}}\right\}_{g \in G}$. Thus, if $g, g^{\prime} \in G$,

$$
\begin{aligned}
& g^{\prime} \in g H_{z_{0}} \Longleftrightarrow g H_{z_{0}} \cap g^{\prime} H_{z_{0}} \neq \emptyset \Longleftrightarrow g H_{z_{0}}=g^{\prime} H_{z_{0}} ; \\
& g^{\prime} \in g \Sigma_{z_{0}} \Longleftrightarrow g \Sigma_{z_{0}} \cap g^{\prime} \Sigma_{z_{0}} \neq \emptyset \Longleftrightarrow g \Sigma_{z_{0}}=g^{\prime} \Sigma_{z_{0}} .
\end{aligned}
$$


Given $g \in G$, we will call $g H_{z_{0}}\left(g \Sigma_{z_{0}}\right)$ an integral manifold for $\mathfrak{h}_{z_{0}}\left(\mathfrak{X}_{z_{0}}\right)$ in $G$ passing through $g$. Since $H_{z_{0}}$ is closed and connected in $G$, we have the following result on the integral manifolds for $\mathfrak{h}_{z_{0}}$ : if we fix $g_{0} \in G$, then there exists a neighborhood $V$ of $g_{0}$ in $G$ such that for $g, g^{\prime} \in V$,

$$
\left[\left(g H_{z_{0}}\right) \cap V\right] \cap\left[\left(g^{\prime} H_{z_{0}}\right) \cap V\right]=\emptyset \quad \Longrightarrow \quad\left(g H_{z_{0}}\right) \cap\left(g^{\prime} H_{z_{0}}\right)=\emptyset .
$$

The analogous property does not necessarily hold for $\Sigma_{z_{0}}$ since $\Sigma_{z_{0}}$ is not necessarily closed in $G$.

Fix $g^{0} \in G$. From item 3. in the discussion of the Frobenius theorem in section 5 , we have the following canonical local coordinates $\left(\Phi_{0}, V_{0}, \Delta_{0}\right)$ of $g^{0}$ in $G$, which we call $F$-local coordinates of $g^{0}$ :

i) $V_{0}$ is a neighborhood of $g^{0}$ in $G$;

ii) $\Delta_{0}=\Delta_{0}^{\prime} \times \Delta_{0}^{\prime \prime} \times \Delta_{0}^{\prime \prime \prime} \subset \mathbb{C}^{m_{2}} \times \mathbb{C}^{m_{1}} \times \mathbb{C}^{m_{0}}$ where

$$
\Delta_{0}^{\prime}=\prod_{i=1}^{m_{2}}\left\{\left|\xi_{i}\right|<r_{i}\right\}, \quad \Delta_{0}^{\prime \prime}=\prod_{i=1}^{m_{1}}\left\{\left|\eta_{i}\right|<r_{i}\right\}, \quad \Delta_{0}^{\prime \prime \prime}=\prod_{i=1}^{m_{0}}\left\{\left|\zeta_{i}\right|<r_{i}\right\} ;
$$

iii) $\Phi_{0}: g \in V_{0} \mapsto(\xi(g), \eta(g), \zeta(g)) \in \Delta_{0}$ is a holomorphic isomorphism with $\Phi_{0}\left(g^{0}\right)=0 \in \Delta_{0}$

which satisfies the properties:

i') given $\xi^{0}=\left(\xi_{1}^{0}, \ldots, \xi_{m_{2}}^{0}\right) \in \Delta_{0}^{\prime}$, the $\left(m_{0}+m_{1}\right)$-dimensional polydisk in $\Delta_{0}$ defined by the equations

$$
S_{\xi_{0}}: \quad \xi_{i}=\xi_{i}^{0}, \quad i=1, \ldots, m_{2},
$$

corresponds to an integral manifold for $\mathfrak{X}_{0}$ from the map $\Phi_{0}$, i.e., if $g \in \Phi_{0}^{-1}\left(S_{\xi_{0}}\right)$, then $\Phi_{0}^{-1}\left(S_{\xi_{0}}\right)=$ the connected component of $\left[g \Sigma_{z_{0}}\right] \cap V_{0}$ containing $g$;

ii') given $\left(\xi^{0}, \eta^{0}\right)=\left(\xi_{1}^{0}, \ldots, \xi_{m_{2}}^{0} ; \eta_{1}^{0}, \ldots, \eta_{m_{1}}^{0}\right) \in \Delta_{0}^{\prime} \times \Delta_{0}^{\prime \prime}$, the $m_{0}$-dimensional polydisk in $\Delta_{0}$ defined by the equations

$$
S_{\left(\xi^{0}, \eta^{0}\right)}: \quad \xi_{i}=\xi_{i}^{0}, \eta_{j}=\eta_{j}^{0}, \quad i=1, \ldots, m_{2} ; j=1, \ldots, m_{1},
$$

corresponds to an integral manifold for $\mathfrak{h}_{0}$ from the map $\Phi_{0}$, i.e., if $g \in \Phi_{0}^{-1}\left(S_{\left(\xi^{0}, \eta^{0}\right)}\right)$, then $\Phi_{0}^{-1}\left(S_{\left(\xi^{0}, \eta^{0}\right)}\right)=\left[g H_{z_{0}}\right] \cap V_{0}$.

From property ii') we have:

iii') fix $\left(\xi^{0}, \eta^{0}\right),\left(\xi^{1}, \eta^{1}\right) \in \Delta_{0}^{\prime} \times \Delta_{0}^{\prime \prime}$, and let $g^{0} \in \Phi_{0}^{-1}\left(S_{\left(\xi^{0}, \eta^{0}\right)}\right)$ and $g^{1} \in$ $\Phi_{0}^{-1}\left(S_{\left(\xi^{1}, \eta^{1}\right)}\right)$. If $\left(\xi^{0}, \eta^{0}\right) \neq\left(\xi^{1}, \eta^{1}\right)$ then $g^{0} H_{z_{0}} \cap g^{1} H_{z_{0}}=\emptyset$. 
Using the local coordinate system $F=\left\{\left(\Phi_{0}, V_{0}, \Delta_{0}\right)\right\}_{g^{0} \in G}$ for $G$ at $g^{0}$, we canonically obtain a local coordinate system $\mathbf{f}=\left\{\left(\phi_{0}, v_{0}, \Delta_{0}^{\prime} \times \Delta_{0}^{\prime \prime}\right)\right\}_{z^{0} \in M}$ for $M$ at a point $z^{0}$ as follows: fix $z^{0} \in M$ and take $g^{0} \in G$ with $\psi_{z_{0}}\left(g^{0}\right)=$ $g^{0}\left(z_{0}\right)=z^{0}$. Then we have $F$-local coordinates $\left(\Phi_{0}, V_{0}, \Delta_{0}\right)$ at $g^{0}$ for $G$. We set $v_{0}=\psi_{z_{0}}\left(V_{0}\right) \subset M$; this is a neighborhood of $z^{0}$ in $M$. Take $z^{\prime} \in v_{0}$ and choose $g^{\prime} \in V_{0}$ such that $\psi_{z_{0}}\left(g^{\prime}\right)=z^{\prime}$. Let $\Phi_{0}\left(g^{\prime}\right)=\left(\xi^{\prime}, \eta^{\prime}, \zeta^{\prime}\right) \in \Delta_{0}$ and consider the correspondence

$$
\phi_{0}: z^{\prime} \mapsto\left(\xi^{\prime}, \eta^{\prime}\right) \in \Delta_{0}^{\prime} \times \Delta_{0}^{\prime \prime} .
$$

Since $M=G / H_{z_{0}}$, we see from condition ii') that $\phi_{0}\left(z^{\prime}\right)$ does not depend on the choice of $g^{\prime} \in V_{0}$ with $\psi_{z_{0}}\left(g^{\prime}\right)=z^{\prime}$. Thus $\phi_{0}$ defines a mapping from $v_{0}$ onto $\Delta_{0}^{\prime} \times \Delta_{0}^{\prime \prime}$. We also see from iii') that $\phi_{0}$ is one-to-one. Moreover, $\phi_{0}$ is holomorphic. To see this, we consider a holomorphic section $\tau$ of $G$ over $v_{0}$ via $\psi_{z_{0}}$ such that

$$
\tau: z^{\prime} \in v_{0} \mapsto \tau\left(z^{\prime}\right) \in V_{0}
$$

and $\tau\left(z^{0}\right)=g^{0}$. We let $\Phi_{0}\left(\tau\left(z^{\prime}\right)\right)=\left(\xi\left(z^{\prime}\right), \eta\left(z^{\prime}\right), \zeta\left(z^{\prime}\right)\right) \in \Delta_{0}$, so that $z^{\prime} \rightarrow$ $\left(\xi\left(z^{\prime}\right), \eta\left(z^{\prime}\right), \zeta\left(z^{\prime}\right)\right)$ is a holomorphic mapping from $v_{0}$ into $\Delta_{0}$. On the other hand, by the definition of $\phi_{0}$ we have $\phi_{0}\left(z^{\prime}\right)=\left(\xi\left(z^{\prime}\right), \eta\left(z^{\prime}\right)\right) \in \Delta_{0}^{\prime} \times \Delta_{0}^{\prime \prime}$. Hence $\phi_{0}$ is holomorphic on $v_{0}$. Thus the family $\mathrm{f}:=\left\{\left(\phi_{0}, v_{0}, \Delta_{0}^{\prime} \times \Delta_{0}^{\prime \prime}\right)\right\}_{z^{0} \in M}$ gives a local coordinate system for $M$. We will call $\left(\phi_{0}, v_{0}, \Delta_{0}^{\prime} \times \Delta_{0}^{\prime \prime}\right) F$-local coordinates at $z^{0}$ for $M$ via $g^{0}$ (i.e., via $F$-local coordinates $\left(\Phi_{0}, V_{0}, \Delta_{0}\right)$ at $\left.g^{0}\right)$.

We recall from (6.16) the subset $\sigma_{z_{0}}:=\psi_{z_{0}}\left(\Sigma_{z_{0}}\right)=\Sigma_{z_{0}}\left(z_{0}\right)$ of $M$. Then:

(1') $\sigma_{z_{0}}$ is a parabolic, irreducible, $m_{1}$-dimensional non-singular f-generalized analytic set in D. Moreover, $\Sigma_{z_{0}}=G \cap$ Aut $\sigma_{z_{0}} ; \Sigma_{z_{0}}$ acts transitively on $\sigma_{z_{0}}$; and $\sigma_{z_{0}} \approx \Sigma_{z_{0}} / H_{z_{0}}$.

(2') $g \sigma_{z_{0}}=\psi_{z_{0}}\left(g \Sigma_{z_{0}}\right)$ for $g \in G$. Moreover, $M=\cup_{g \in G} g \sigma_{z_{0}}$ satisfies the property that if $g_{1} \sigma_{z_{0}} \cap g_{2} \sigma_{z_{0}} \neq \emptyset$ for $g_{1}, g_{2} \in G$ then $g_{1} \sigma_{z_{0}}=g_{2} \sigma_{z_{0}}$.

(3') fix $z^{0} \in M$ and take $g^{0} \in G$ with $\psi_{z_{0}}\left(g^{0}\right)=z^{0}$. Let $\left(\phi_{0}, v_{0}, \Delta_{0}^{\prime} \times \Delta_{0}^{\prime \prime}\right)$ be $F$-local coordinates at $z^{0}$ for $M$ via $g^{0}$. Take $z^{\prime} \in v_{0}$ with $\phi_{0}\left(z^{\prime}\right)=$ $\left(\xi^{\prime}, \eta^{\prime}\right) \in \Delta_{0}^{\prime} \times \Delta_{0}^{\prime \prime}$ and let $g^{\prime} \in G$ with $z^{\prime} \in g^{\prime} \sigma_{z_{0}}$. Then there exists a connected component $\sigma^{\prime}$ of $\left[g^{\prime} \sigma_{z_{0}}\right] \cap v_{0}$ such that $\sigma^{\prime}=\phi_{0}^{-1}\left(\left\{\xi^{\prime}\right\} \times \Delta_{0}^{\prime \prime}\right)$ in $M$.

Proof. The first assertion in (2') follows from the definition of $\sigma_{z_{0}}$. We next verify (3'). Let $z^{\prime} \in v_{0}$ with $\phi_{0}\left(z^{\prime}\right)=\left(\xi^{\prime}, \eta^{\prime}\right)$ and let $g^{\prime} \in G$ with $z^{\prime} \in$ $g^{\prime} \sigma_{z_{0}}$. We take $h^{\prime} \in V_{0}$ with $\psi_{z_{0}}\left(h^{\prime}\right)=h^{\prime}\left(z_{0}\right)=z^{\prime}$; then $\Phi_{0}\left(h^{\prime}\right)=\left(\xi^{\prime}, \eta^{\prime}, \zeta^{\prime}\right)$ for some $\zeta^{\prime} \in \Delta_{0}^{\prime \prime \prime}$. Since $z^{\prime} \in g^{\prime} \sigma_{z_{0}}$, we can find $s \in \Sigma_{z_{0}}$ such that $z^{\prime}=g^{\prime} s\left(z_{0}\right)$, 
and $\left(h^{\prime}\right)^{-1} g^{\prime} s \in H_{z_{0}}$. Since $\Sigma_{z_{0}}$ is a connected Lie subgroup of $G$ which contains $H_{z_{0}}$, it follows that

$$
g^{\prime} \Sigma_{z_{0}}=g^{\prime} s \Sigma_{z_{0}} \subset h^{\prime} H_{z_{0}} \cdot \Sigma_{z_{0}}=h^{\prime} \Sigma_{z_{0}} \quad \text { so that } \quad g^{\prime} \Sigma_{z_{0}}=h^{\prime} \Sigma_{z_{0}} .
$$

Since $\Phi_{0}\left(h^{\prime}\right)=\left(\xi^{\prime}, \eta^{\prime}, \zeta^{\prime}\right)$, we see from i') that the connected component of $\left[g^{\prime} \Sigma_{z_{0}}\right] \cap V_{0}=\left[h^{\prime} \Sigma_{z_{0}}\right] \cap V_{0}$ containing $h^{\prime}$ is equal to $\Phi_{0}^{-1}\left(\left\{\xi^{\prime}\right\} \times \Delta_{0}^{\prime \prime} \times \Delta_{0}^{\prime \prime \prime}\right)$. Together with the observation that $\Phi_{0}^{-1}(\xi, \eta, \zeta)\left(z_{0}\right)=\phi_{0}^{-1}(\xi, \eta)$ for $(\xi, \eta, \zeta) \in$ $\Delta_{0}$, this yields (3').

We now prove the first assertion in (1'). Fix $z^{0} \in \sigma_{z_{0}}$ and let $g^{0} \in G$ with $g^{0}\left(z_{0}\right)=z^{0}$. As before, we consider $F$-local coordinates $\left(\Phi_{0}, V_{0}, \Delta_{0}\right)$ at $g^{0}$ for $G$ and $\left(\phi_{0}, v_{0}, \Delta_{0}^{\prime} \times \Delta_{0}^{\prime \prime}\right)$ at $z^{0}$ for $M$ via $g^{0}$. Since $\phi_{0}\left(z^{0}\right)=(0,0) \in \Delta_{0}^{\prime} \times \Delta_{0}^{\prime \prime}$ and $z^{0} \in \sigma_{z_{0}}$, it follows from $\left(3^{\prime}\right)$ that $\phi_{0}^{-1}\left(\{0\} \times \Delta_{0}^{\prime \prime}\right)$ coincides with some connected component $\sigma^{\prime}$ of $\left[\sigma_{z_{0}}\right] \cap v_{0}$. Thus, $\sigma^{\prime}$ is an $m_{1}$-dimensional nonsingular analytic set in $v_{0}$. Now $\Sigma_{z_{0}}$ is connected; this implies that $\sigma_{z_{0}}$ is an irreducible $m_{1}$-dimensional non-singular $f$-generalized analytic set in $M$. Furthermore, using the representation for $\Sigma_{z_{0}}$ in (6.18), one can show that $\sigma_{z_{0}}=\Sigma_{z_{0}}\left(z_{0}\right)$ is parabolic. Now take $g \in D\left(z_{0}\right)$. Since $\lambda(z)$ is continuous on $D$, it follows from Lemma 6.3 and (6.18) that

$$
\lambda(z)=\text { const. }=\lambda\left(g\left(z_{0}\right)\right) \quad \text { for } z \in g \sigma_{z_{0}} .
$$

Since $-\lambda(z)$ is an exhaustion function for $D$, we must have $g \sigma_{z_{0}} \Subset D$, proving the first assertion in $\left(1^{\prime}\right)$.

To prove the second assertion in (2'), assume that $g^{\prime} \sigma_{z_{0}} \cap g^{\prime \prime} \sigma_{z_{0}} \neq \emptyset$ and take $z^{0} \in g^{\prime} \sigma_{z_{0}} \cap g^{\prime \prime} \sigma_{z_{0}}$. Then there exist $s^{\prime}, s^{\prime \prime} \in \Sigma_{z_{0}}$ with $z^{0}=g^{\prime} s^{\prime}\left(z_{0}\right)=$ $g^{\prime \prime} s^{\prime \prime}\left(z_{0}\right)$, so that we find $h \in H_{z_{0}}$ such that $g^{\prime} s^{\prime}=g^{\prime \prime} s^{\prime \prime} h$. Since $\Sigma_{z_{0}}$ is a group containing $H_{z_{0}}$, it follows that $g^{\prime}=g^{\prime \prime} s^{\prime \prime} h\left(s^{\prime}\right)^{-1} \in g^{\prime \prime} \Sigma_{z_{0}}$, and hence $g^{\prime} \Sigma_{z_{0}} \subset g^{\prime \prime} \Sigma_{z_{0}}$. We thus have $g^{\prime} \sigma_{z_{0}} \subset g^{\prime \prime} \sigma_{z_{0}}$, and hence $g^{\prime} \sigma_{z_{0}}=g^{\prime \prime} \sigma_{z_{0}}$.

For the second assertion in (1'), since $G \subset$ Aut $M$, it suffices to prove that $\left\{g \in G: g \sigma_{z_{0}}=\sigma_{z_{0}}\right\}=\Sigma_{z_{0}}$ and to verify that $\Sigma_{z_{0}}$ acts transitively on $\sigma_{z_{0}}$. Let $g \in \Sigma_{z_{0}}$. We have $g \sigma_{z_{0}}=\sigma_{z_{0}}$ by (2') which shows that $\Sigma_{z_{0}} \subset\{g \in$ $\left.G: g \sigma_{z_{0}}=\sigma_{z_{0}}\right\}$. To prove the reverse inclusion, fix $g \in G$ with $g \sigma_{z_{0}}=\sigma_{z_{0}}$. There exist $s \in \Sigma_{z_{0}}$ and $h \in H_{z_{0}}$ such that $g=s h$. Since $H_{z_{0}} \subset \Sigma_{z_{0}}$, it follows that $g \in \Sigma_{z_{0}}$, which proves $\left\{g \in G: g \sigma_{z_{0}}=\sigma_{z_{0}}\right\} \subset \Sigma_{z_{0}}$. To verify the transitivity, let $\zeta \in \sigma_{z_{0}}$. From (6.2) we can find $g \in D\left(z_{0}\right)$ with $g\left(z_{0}\right)=\zeta$. Using (2') we have $g \sigma_{z_{0}}=\sigma_{z_{0}}$ so that $g \in \Sigma_{z_{0}}$ as required.

Using (2'), (3') and (6.2) we have

$$
M=\bigcup_{g \in G} g \sigma_{z_{0}} \quad \text { and } \quad D=\bigcup_{g \in D\left(z_{0}\right)} g \sigma_{z_{0}},
$$

which provide foliations of $M$ and $D$. We see from (1') that each leaf $g \sigma_{z_{0}}$ in $D$ for $g \in D\left(z_{0}\right)$ is relatively compact in $D$, and hence each leaf $g \sigma_{z_{0}}$ in $M$ 
for $g \in G$ is relatively compact in $M$. Thus (i) in $1-a$. is proved. Assertions (1') and (2') verify (o) in $1-a$.

To prove (ii) in $1-a$. and $1-b$., we begin with the following two items:

$\alpha$. Let $z^{\prime} \in D$ and let $g^{\prime} \in G$ with $g^{\prime}\left(z_{0}\right)=z^{\prime}$. Let $l$ be a one-dimensional complex analytic curve passing through $z^{\prime}$ and contained in a neighborhood $U$ of $z^{\prime}$ in $M$ such that $\lambda(z)$ is constant on $l$. Then $l \subset g^{\prime} \sigma_{z_{0}}$.

$\beta . \Sigma_{z_{0}}$ is closed in $G$ if and only if $\sigma_{z_{0}}$ is closed in $M$.

Proof. We prove $\alpha$. by contradiction. It suffices to consider the case when $l$ intersects the $m_{1}$-dimensional generalized analytic set $g^{\prime} \sigma_{z_{0}}$ transversally at $z^{\prime}$. Indeed, assume that $\alpha$. is true for any $g^{\prime} \sigma_{z_{0}}$ which $l$ intersects transversally. Now assume that there exists some set $g^{\prime} \sigma_{z_{0}}$ such that $l$ does not intersect $g^{\prime} \sigma_{z_{0}}$ transversally at $z^{\prime}$. To verify $\alpha$. in such a case, proceeding by contradiction, we assume that $l \not \subset g^{\prime} \sigma_{z_{0}}$. Since we have a foliation $D=\cup_{g \in D\left(z_{0}\right)} g \sigma_{z_{0}}$ of $D$ in (6.21), we can find $g^{\prime \prime} \in D\left(z_{0}\right)$ sufficiently close to $g^{\prime}$ such that $g^{\prime \prime} \sigma_{z_{0}} \cap l \neq \emptyset ; g^{\prime \prime} \sigma_{z_{0}} \cap g^{\prime} \sigma_{z_{0}}=\emptyset$; and $l$ intersects $g^{\prime \prime} \sigma_{z_{0}}$ transversally. But then by assumption we have $l \subset g^{\prime \prime} \sigma_{z_{0}}$, which is a contradiction.

Thus we assume that $l \not \subset g^{\prime} \sigma_{z_{0}}$ and also that $l$ intersects $g^{\prime} \sigma_{z_{0}}$ transversally at $z^{\prime}$. Using standard results for homgeneous spaces, we can choose $X \in \mathfrak{X}$ such that the curve $C:=\left\{\exp t X\left(z^{\prime}\right): t \in \mathbb{C}\right\}$ in $M$ has the same complex tangent direction at $z^{\prime}$ as the line $l$. Call this direction $a$. Since $\lambda(z)=$ const. on $l$, we have $\left.\left[\frac{\partial^{2} \lambda\left(z^{\prime}+a t\right)}{\partial t \partial \bar{t}}\right]\right|_{t=0}=0$. It follows from the equivalent conditions o. $\sim$ iii. before Lemma 6.3 that $\lambda\left(\exp t X\left(z^{\prime}\right)\right)=$ const. for $t \in \mathbb{C}$. Since $\left(g^{\prime}\right)^{-1}\left(z^{\prime}\right)=z_{0} \in D$, we have $\left(g^{\prime}\right)^{-1} \in D\left(z^{\prime}\right)=D^{\prime}\left(z^{\prime}\right)$. Thus, from property c., we see that $\lambda\left(\left(g^{\prime}\right)^{-1} \exp t X\left(z^{\prime}\right)\right)=$ const. for $t \in \mathbb{C}$. However the curve $C^{\prime}:=\left(g^{\prime}\right)^{-1} C$ intersects $\sigma_{z_{0}}$ transversally at $z_{0}$ since the curve $C$ intersects $g^{\prime} \sigma_{z_{0}}$ transversally at $z^{\prime}$. We can choose $Y \in \mathfrak{X}$ such that the curve $\widehat{C}:=$ $\left\{\exp t Y\left(z_{0}\right): t \in \mathbb{C}\right\}$ has the same complex tangent direction at $z_{0}$ as $C^{\prime}$. This shows that $\widehat{C} \not \subset \sigma_{z_{0}}$. We again use the conditions o. $\sim$ iii. to conclude that $\lambda(z)=$ const. on $\widehat{C}$, so that $Y \in \mathfrak{X}_{z_{0}}$. Since $\sigma_{z_{0}}=\Sigma_{z_{0}}\left(z_{0}\right)$ and $\{\exp t Y$ : $t \in \mathbb{C}\} \subset \Sigma_{z_{0}}$, we have $\widehat{C} \subset \sigma_{z_{0}}$. This is a contradiction.

To prove the sufficiency in $\beta$., let $z^{\nu} \in \sigma_{z_{0}}, \nu=1,2, \ldots$, and let $z^{0} \in D$ with $z^{\nu} \rightarrow z^{0}$ as $\nu \rightarrow \infty$. We show that $z^{0} \in \sigma_{z_{0}}$. Fix $g^{0} \in G$ with $g^{0}\left(z_{0}\right)=z^{0}$. Consider $F$-local coordinates $\left(\Phi_{0}, V_{0}, \Delta_{0}\right)$ at $g^{0}$ for $G$ and $\left(\phi_{0}, v_{0}, \Delta_{0}^{\prime} \times \Delta_{0}^{\prime \prime}\right)$ at $z^{0}$ for $M$ via $g^{0}$. Since $z^{\nu} \in v_{0}$ for sufficiently large $\nu$, we can find $g^{\nu} \in V_{0}$ with $g^{\nu}\left(z_{0}\right)=z^{\nu}$. By taking a subsequence of $\left\{g^{\nu}\right\}_{\nu}$ if necessary, we may assume that there exists $g^{*} \in \overline{V_{0}} \subset G$ such that $g^{\nu} \rightarrow g^{*}$ as $\nu \rightarrow \infty$, so that $g^{*}\left(z_{0}\right)=z^{0}$. On the other hand, since $z^{\nu} \in \sigma_{z_{0}}$, we can find $s^{\nu} \in \Sigma_{z_{0}}$ such that $z^{\nu}=s^{\nu}\left(z_{0}\right), \nu=1,2, \ldots$. Hence $g^{\nu}\left(z_{0}\right)=s^{\nu}\left(z_{0}\right)$, so that $\left(s^{\nu}\right)^{-1} g^{\nu} \in$ 
$H_{z_{0}} \subset \Sigma_{z_{0}}$. Thus $g^{\nu} \in s^{\nu} \Sigma_{z_{0}}=\Sigma_{z_{0}}$. Since $g^{\nu} \rightarrow g^{*}$ as $\nu \rightarrow \infty$ and since $\Sigma_{z_{0}}$ is assumed to be closed in $G$, it follows that $g^{*} \in \Sigma_{z_{0}}$, so that $z^{0}=g^{*}\left(z_{0}\right) \in \sigma_{z_{0}}$. To prove the necessity in $\beta$., let $g^{\nu} \in \Sigma_{z_{0}}, \nu=1,2, \ldots$ and $g^{0} \in G$ with $g^{\nu} \rightarrow g^{0}$ as $\nu \rightarrow \infty$. We show that $g^{0} \in \Sigma_{z_{0}}$. We set $z^{\nu}=g^{\nu}\left(z_{0}\right) \in \sigma_{z_{0}}$ and $z^{0}=g^{0}\left(z_{0}\right) \in M$. Since $z^{\nu} \rightarrow z^{0}$ as $\nu \rightarrow \infty$ and since $\sigma_{z_{0}}$ is assumed to be closed in $M$, it follows that $z^{0} \in \sigma_{z_{0}}$. Thus we can find $s^{0} \in \Sigma_{z_{0}}$ with $s^{0}\left(z_{0}\right)=z^{0}$. Hence $g^{0}\left(z_{0}\right)=s^{0}\left(z_{0}\right)$, so that $g^{0} \in s^{0} H_{z_{0}} \subset s^{0} \Sigma_{z_{0}}=\Sigma_{z_{0}}$ and $\beta$. is proved.

Since $-\lambda(z)$ is a plurisubharmonic exhaustion function for $D$, assertion $\alpha$. implies (ii) of $1-a$., completing the proof of $1-a$.

To prove $1-b$. assume that $\sigma_{z_{0}}$ is closed in $M$. From $1-a$., $\sigma_{z_{0}}$ is an irreducible, $m_{1}$-dimensional compact non-singular analytic set in $D$. From $\beta ., \Sigma_{z_{0}}$ is closed, which proves (o) in $1-b$.

To prove (i) in $1-b$. we note that $\Sigma_{z_{0}}$ is closed in $G$, so that $\Sigma_{z_{0}}$ is an $\left(m_{0}+m_{1}\right)$-dimensional connected closed Lie subgroup of $G$ which corresponds to the Lie subalgebra $\mathfrak{X}_{z_{0}}$ of $\mathfrak{X}$. We consider the quotient space $M_{0}:=G / \Sigma_{z_{0}}$; this is an $m_{2}$-dimensional complex homogeneous space with Lie transformation group $G$ which acts transitively on $M_{0}$. Since $H_{z_{0}} \subset \Sigma_{z_{0}}$ as Lie groups, we have the canonical holomorphic projection

$$
\pi_{0}: z=g H_{z_{0}} \in M \mapsto w=\pi_{0}(z)=g \Sigma_{z_{0}} \in M_{0} .
$$

Just as we identified $H_{z_{0}} \subset G$ with the point $z_{0}$ in $M$, we may consider $w_{0}:=\Sigma_{z_{0}}$ as a point in $M_{0}$, i.e., $\Sigma_{z_{0}}$ is the isotropy subgroup of $G$ for the point $w_{0}$ in $M_{0}$. Then

$$
\begin{aligned}
& \pi_{0}\left(g\left(z_{0}\right)\right)=g\left(w_{0}\right), \quad \text { for all } g \in G \text {; } \\
& \pi_{0}^{-1}\left(g\left(w_{0}\right)\right)=g \sigma_{z_{0}}, \quad \text { for all } g \in G \text {. }
\end{aligned}
$$

Indeed, the first formula comes from the definition of $\pi_{0}$. For the second one, we first note that

$$
\pi_{0}\left(g \sigma_{z_{0}}\right)=\pi_{0}\left(g \Sigma_{z_{0}}\left(z_{0}\right)\right)=g \Sigma_{z_{0}}\left(w_{0}\right)=g\left(w_{0}\right),
$$

which proves the inclusion $g \sigma_{z_{0}} \subset \pi_{0}^{-1}\left(g\left(w_{0}\right)\right)$. To prove the inclusion

$$
\pi_{0}^{-1}\left(g\left(w_{0}\right)\right) \subset g \sigma_{z_{0}}
$$

take $z \in M$ with $\pi_{0}(z)=g\left(w_{0}\right)$. We can find $g^{\prime} \in G$ with $g^{\prime}\left(z_{0}\right)=z$. Thus, $g^{\prime}\left(w_{0}\right)=\pi_{0}\left(g^{\prime}\left(z_{0}\right)\right)=g\left(w_{0}\right)$ so that $g^{\prime} \in g \Sigma_{z_{0}}$. It follows that $g^{\prime}\left(z_{0}\right) \in g \sigma_{z_{0}}$, and $z \in g \sigma_{z_{0}}$. Thus, (6.22) is proved. 
Set $D_{0}:=\pi_{0}(D) \subset M_{0}$. We have $D_{0} \Subset M_{0}$ since $D \Subset M$; moreover, let $w \in D_{0}\left(\partial D_{0}\right)$ and take $g \in G$ such that $w=g\left(w_{0}\right)$. Then

$$
\pi_{0}^{-1}(w)=g \sigma_{z_{0}} \Subset D(\partial D) .
$$

Indeed, since the proof is similar, we only give the proof in the case $w \in D_{0}$. Since $g\left(z_{0}\right) \in D$, we find $g^{\prime} \in D\left(z_{0}\right)$ such that $g\left(z_{0}\right)=g^{\prime}\left(z_{0}\right)$, which implies $g=g^{\prime} h \in D\left(z_{0}\right)$. This together with (6.22) and (i) in 1-a. implies (6.23).

Therefore, we have

$$
D=\pi_{0}^{-1}\left(D_{0}\right) \quad \text { and } \quad \partial D=\pi_{0}^{-1}\left(\partial D_{0}\right) .
$$

To prove that $D_{0}$ is a Stein domain in $M_{0}$, we define a real-valued function $\lambda_{0}(w)$ on $D_{0}$ as follows. Fix $w \in D_{0}$ and take $z \in D$ with $\pi_{0}(z)=w$. We define $\lambda_{0}(w):=\lambda(z)$, which is well-defined by (6.23). In order to prove that $\lambda_{0}(w)$ is a strictly plurisubharmonic exhaustion function on $D_{0}$ we shall utilize the usual coordinates for $M$ and $M_{0}$. Fixing $g^{0} \in G$, by the Frobenius theorem we have $F$-local coordinates $\left(\Phi_{0}, V_{0}, \Delta_{0}\right)$ at $g^{0}$ for $G$ with the following properties:

$$
\Phi_{0}: g \in V_{0} \mapsto(\xi(g), \eta(g), \zeta(g)) \in \Delta_{0}:=\Delta_{0}^{\prime} \times \Delta_{0}^{\prime \prime} \times \Delta_{0}^{\prime \prime \prime} ;
$$

for a given $p=\left(\xi^{\prime}, \eta^{\prime}, \zeta^{\prime}\right) \in \Delta_{0}$, if we set $g^{\prime}:=\Phi_{0}^{-1}(p) \in V_{0}$, then

$$
\begin{aligned}
& {\left[g^{\prime} H_{z_{0}}\right] \cap V_{0}=\Phi_{0}^{-1}\left(\left\{\left(\xi^{\prime}, \eta^{\prime}\right)\right\} \times \Delta_{0}^{\prime \prime \prime}\right)} \\
& {\left[g^{\prime} \Sigma_{z_{0}}\right] \cap V_{0}=\Phi_{0}^{-1}\left(\left\{\xi^{\prime}\right\} \times \Delta_{0}^{\prime \prime} \times \Delta_{0}^{\prime \prime \prime}\right)}
\end{aligned}
$$

and

$$
\begin{aligned}
& \left(\left[g^{\prime} H_{z_{0}}\right] \cap V_{0}\right) \cap\left(\left[g^{\prime \prime} H_{z_{0}}\right] \cap V_{0}\right)=\emptyset \Longrightarrow\left[g^{\prime} H_{z_{0}}\right] \cap\left[g^{\prime \prime} H_{z_{0}}\right]=\emptyset ; \\
& \left(\left[g^{\prime} \Sigma_{z_{0}}\right] \cap V_{0}\right) \cap\left(\left[g^{\prime \prime} \Sigma_{z_{0}}\right] \cap V_{0}\right)=\emptyset \Longrightarrow\left[g^{\prime} \Sigma_{z_{0}}\right] \cap\left[g^{\prime \prime} \Sigma_{z_{0}}\right]=\emptyset .
\end{aligned}
$$

Note that $\Sigma_{z_{0}}$ and $H_{z_{0}}$ are closed in $G$. We set $U_{1}:=\left\{g H_{z_{0}} \in G: g \in V_{0}\right\} \subset$ $G$, and we regard $U_{1}$ as a neighborhood of $z^{0}=g^{0} H_{z_{0}}$ in $M$. Similarly, we regard $U_{2}:=\left\{g \Sigma_{z_{0}} \in G: g \in V_{0}\right\} \subset G$ as a neighborhood of $w^{0}=g^{0} \Sigma_{z_{0}}$ in $M_{0}$. For $g \in V_{0}$, we consider the correspondences

$$
\begin{array}{ll}
\varphi_{1}=\phi_{0} & : z=g H_{z_{0}} \in U_{1} \mapsto(\xi, \eta) \in \Delta_{0}^{\prime} \times \Delta_{0}^{\prime \prime} ; \\
\varphi_{2} & : w=g \Sigma_{z_{0}} \in U_{2} \mapsto \xi \in \Delta_{0}^{\prime} .
\end{array}
$$

Then $\left(\varphi_{1}, U_{1}, \Delta_{0}^{\prime} \times \Delta_{0}^{\prime \prime}\right)$ and $\left(\varphi_{2}, U_{2}, \Delta_{0}^{\prime}\right)$ define local coordinates at $z^{0}$ for $M$ and local coordinates at $w^{0}$ for $M_{0}$. In particular,

$$
\varphi_{1}\left(\left[g^{0} \sigma_{z_{0}}\right] \cap U_{1}\right)=\{0\} \times \Delta_{0}^{\prime \prime} ; \quad \varphi_{2}\left(\left[g^{0} \Sigma_{z_{0}}\right] \cap U_{2}\right)=0 .
$$


In terms of the local coordinates (6.27), if we restrict $\pi_{0}$ from the neighborhood $U_{1}$ of $z^{0}$ in $M$ to the neighborhood $U_{2}$ of $w^{0}$ in $M_{0}$, we have

$$
\pi_{0}:(\xi, \eta) \in \Delta_{0}^{\prime} \times \Delta_{0}^{\prime \prime} \mapsto \xi \in \Delta_{0}^{\prime} .
$$

From the local coordinate expression for $\pi_{0}$ and (6.24), we have that $\partial D_{0}$ is smooth in $M_{0}$. Moreover, using the local coordinates (6.27), $\lambda_{0}(w)$ is of the form $\lambda_{0}(\xi)=\lambda(\xi, \eta)$ for $(\xi, \eta) \in \Delta_{0}^{\prime} \times \Delta_{0}^{\prime \prime}$. In particular, $\lambda_{0}(\xi)=\lambda(\xi, 0)$ in $\Delta_{0}^{\prime}$. It follows from the plurisubharmonicity of $-\lambda(z)$ on $D$ that $-\lambda_{0}(w)$ is plurisubharmonic on $D_{0}$. Since $\partial D_{0}=\pi_{0}(\partial D)$, the fact that $-\lambda(z)$ is an exhaustion function for $D$ implies that $-\lambda_{0}(w)$ is an exhaustion function for $D_{0}$.

It remains to verify that $\lambda_{0}(w)$ is strictly plurisubharmonic on $D_{0}$. If not, there exists $w^{0} \in D_{0}$ and $a_{0} \in \mathbb{C}^{m_{2}}\left(a_{0} \neq 0\right)$ such that $\left.\left[\frac{\partial^{2} \lambda_{0}\left(w^{0}+a_{0} t\right)}{\partial t \partial \bar{t}}\right]\right|_{t=0}=0$. Take $z^{0} \in D$ and $g^{0} \in D\left(z_{0}\right)$ with $g^{0}\left(z_{0}\right)=z^{0}$ and $\pi_{0}\left(z^{0}\right)=w^{0}$. Using local coordinates $\left(\varphi_{1}, U_{1}, \Delta_{0}^{\prime} \times \Delta_{0}^{\prime \prime}\right)$ at $z^{0}$ for $M$ and $\left(\varphi_{2}, U_{2}, \Delta_{0}^{\prime}\right)$ at $w^{0}$ for $M_{0}$, we may assume that $w^{0}+a_{0} t,|t| \ll 1$ in $U_{2}$ is of the form $\xi=a_{0} t,|t| \ll 1$ in $\Delta_{0}^{\prime}$. Since $\lambda_{0}\left(a_{0} t\right)=\lambda\left(a_{0} t, 0\right),|t| \ll 1$, we have $\left.\left[\frac{\partial^{2} \lambda\left(a_{0} t, 0\right)}{\partial t \partial \bar{t}}\right]\right|_{t=0}=0$. Take $X \in \mathfrak{X}$ with the property that the integral curve $C^{0}:=\left\{\exp t X\left(z^{0}\right): t \in \mathbb{C}\right\}$ in $M$ has direction $\left(a_{0} ; 0\right) \in \mathbb{C}^{m_{1}} \times \mathbb{C}^{m_{2}}$ at $z^{0}$. From the equivalent conditions o. $\sim$ iii. we see that $\lambda(z)=$ const. on $C^{0}$. Since $z^{0} \in C^{0} \cap\left[g^{0} \sigma_{z_{0}}\right]$, it follows from $\alpha$. that $C^{0} \subset g^{0} \sigma_{z_{0}}$. On the other hand, by (6.28), $\left[g^{0} \sigma_{z_{0}}\right] \cap U_{1}$ corresponds to $\{0\} \times \Delta_{0}^{\prime \prime}$ using the local coordinates $\varphi_{1}$. Since the direction of $C^{0}$ at $z^{0}$ is $\left(a_{0} ; 0\right)$ with $a_{0} \neq 0$, this is a contradiction. Condition $1-b$. is proved.

We would like to prove a result, Theorem 6.4, corresponding to Theorem 6.3 in the case when the isotropy subgroup $H_{z}$ is not connected for use in studying special Hopf spaces. We begin with a discussion below which makes clear the difference between the connected case and the non-connnected case. The bulk of the proof of Theorem 6.4 can be found in Appendix B; we commence with some background and motivation. The next two results, Propositions 6.4 and 6.5, are standard. To keep the article self-contained, and since we will use some notation from the proofs later on, we include the brief proofs. One preliminary notational remark: if $A, B$ are subsets (not necessarily subgroups) of a group $G$, we write $A B:=\{a b: a \in A, b \in B\}$.

Proposition 6.4. Let $z_{0} \in M$ and suppose that $H_{z_{0}}$ is not connected. Consider the decomposition of $H_{z_{0}}$ into connected components $H_{z_{0}}^{(k)}, k=1,2, \ldots$, where $H_{z_{0}}^{(1)}=H_{z_{0}}^{\prime}\left(\right.$ see $(6.3)$ ). Then there exists a neighborhood $v_{0}$ of e in $G$ such that

$$
\text { 1. } v_{0} H_{z_{0}}^{(k)} \cap v_{0} H_{z_{0}}^{(l)}=\emptyset, \quad k \neq l ;
$$


2. if $a, b \in v_{0}$ and $a^{-1} b \in H_{z_{0}}$, then $a^{-1} b \in H_{z_{0}}^{\prime}$.

Proof. Since $H_{z_{0}}$ is closed in $G, 2$. automatically holds provided $v_{0}$ is a sufficiently small neighborhood of $e$ in $G$. To verify 1 . we first prove that there exists a neighborhood $v_{0}$ with property 2 . such that

$$
v_{0} H_{z_{0}}^{\prime} \cap\left(\bigcup_{k=2}^{\infty} v_{0} H_{z_{0}}^{(k)}\right)=\emptyset .
$$

If no such neighborhood exists, then for any neighborhood $v_{0}$ of $e$, for $j=$ $1,2, \ldots$, we can find $g_{j} \in v_{0}, h_{j} \in H_{z_{0}}^{\prime}$ and $g_{j k_{j}} \in v_{0}, h_{j k_{j}} \in H_{z_{0}}^{\left(k_{j}\right)}, k_{j} \geq 2$ such that $g_{j}, g_{j k_{j}} \rightarrow e$ as $j \rightarrow \infty$ and $g_{j} h_{j}=g_{j k_{j}} h_{j k_{j}}, j=1,2 \ldots$ Hence $g_{j k_{j}}^{-1} g_{j}=h_{j k_{j}} h_{j}^{-1}$. The left-hand side $g_{j k_{j}}^{-1} g_{j}$ tends to $e$ as $j \rightarrow \infty$ and the right-hand side $h_{j k_{j}} h_{j}^{-1}$ always belongs to $H_{z_{0}}$. Hence $h_{j k_{j}} h_{j}^{-1} \in H_{z_{0}}^{\prime}$ for sufficiently large $j$, so that $h_{j k_{j}} \in H_{z_{0}}^{\prime}$. This contradicts $h_{j k_{j}} \in H_{z_{0}}^{\left(k_{j}\right)}$ for all $k_{j} \geq 2$, verifying (6.29). We now prove that this neighborhood $v_{0}$ satisfies 1. If not, $v_{0} h_{k} H_{z_{0}}^{\prime} \cap v_{0} h_{l} H_{z_{0}}^{\prime} \neq \emptyset$ for some $k \neq l$. Then $v_{0}\left(h_{k} H_{z_{0}}^{\prime} h_{k}^{-1}\right) \cap$ $v_{0}\left(h_{l} h_{k}^{-1}\right)\left(h_{k} H_{z_{0}}^{\prime} h_{k}^{-1}\right) \neq \emptyset$. Since $H_{z_{0}}^{\prime}$ is a normal subgroup of $H_{z_{0}}$, we have $v_{0} H_{z_{0}}^{\prime} \cap v_{0}\left(h_{l} h_{k}^{-1}\right) H_{z_{0}}^{\prime} \neq \emptyset$. Since $k \neq l$ we have $\left(h_{l} h_{k}^{-1}\right) H_{z_{0}}^{\prime}=H_{z_{0}}^{(m)}$ for some $m \geq 2$ and $H_{z_{0}}^{(m)} \cap H_{z_{0}}^{\prime}=\emptyset$ by (6.3). Hence $v_{0} H_{z_{0}}^{\prime} \cap v_{0} H_{z_{0}}^{(m)} \neq \emptyset$, which contradicts (6.29). Condition 1 . is proved.

We consider the quotient space $M^{\prime}=G / H_{z_{0}}^{\prime}$ which is a homogeneous space with Lie transformation group $G$ which acts transitively on $M^{\prime}$. We note that $M^{\prime}$ depends on the point $z_{0}$ in $M$. The space $M^{\prime}$ has the same dimension, $m-n$, as $M$. We write $w_{0}$ for the point in $M^{\prime}$ which corresponds to $H_{z_{0}}^{\prime}$ and we introduce the notation $\widehat{H}_{w_{0}}:=H_{z_{0}}^{\prime} \subset G$. Then we have the projections

$$
\widehat{\psi}_{w_{0}}: g \in G \mapsto g\left(w_{0}\right) \in M^{\prime}, \quad \widehat{\pi}_{w_{0}}: g \in G \mapsto g \widehat{H}_{w_{0}} \in G / \widehat{H}_{w_{0}} .
$$

Recall we defined $\mathfrak{h}_{z_{0}}$ as the Lie subalgebra of $\mathfrak{X}$ which corresponds to $H_{z_{0}}^{\prime}$; we now write $\widehat{\mathfrak{h}}_{w_{0}}:=\mathfrak{h}_{z_{0}} \subset \mathfrak{X}$. Thus, $\widehat{H}_{w_{0}}$ is the connected isotropy subgroup of $G$ for $w_{0} \in M^{\prime}$ whose Lie subalgebra is $\widehat{\mathfrak{h}}_{w_{0}}$, and a point $w$ in $M^{\prime}$ is identified with $g \widehat{H}_{w_{0}}$ where $g\left(w_{0}\right)=w$.

We consider the mapping

$$
\widehat{\pi}: g H_{z_{0}}^{\prime} \in M^{\prime} \mapsto g H_{z_{0}} \in M
$$

We remark that $\widehat{\pi}$ depends on $z_{0}$ in $M$. This mapping is well-defined, holomorphic and surjective. Note that for any $w \in M^{\prime}$ and $g \in G$

$$
\widehat{\pi}(g w)=g \widehat{\pi}(w), \quad \widehat{\pi}^{-1}\left(g H_{z_{0}}\right)=\bigcup_{k=1}^{\infty} g h_{k} \widehat{H}_{w_{0}}
$$


with $h_{k} \in H_{z_{0}}^{(k)}$ as in (6.3) since $H_{z_{0}}^{(k)}=h_{k} \widehat{H}_{w_{0}}, k=1,2, \ldots$ by (6.3).

To verify (6.30), since $w=g^{\prime} H_{z_{0}}^{\prime}$ for some $g^{\prime} \in G$, we have, by definition, $\widehat{\pi}(g w)=\widehat{\pi}\left(g g^{\prime} H_{z_{0}}^{\prime}\right)=g g^{\prime} H_{z_{0}}=g \widehat{\pi}(w)$. This proves the first equality. By (6.3) we have $\widehat{\pi}\left(g H_{z_{0}}^{(k)}\right)=g H_{z_{0}}$, so that $\cup_{k=1}^{\infty} g H_{z_{0}}^{(k)} \subset \widehat{\pi}^{-1}\left(g H_{z_{0}}\right)$. Conversely, if we take $h \in G$ such that $\widehat{\pi}\left(h H_{z_{0}}^{\prime}\right)=g H_{z_{0}}$, then we have $g^{-1} h \in H_{z_{0}}=$ $\cup_{k=1}^{\infty} H_{z_{0}}^{(k)}$, and hence $h \in g H_{z_{0}}^{(k)}$ for some $k$. Thus, $h H_{z_{0}}^{\prime} \subset g H_{z_{0}}^{(k)} H_{z_{0}}^{\prime}=g H_{z_{0}}^{(k)}$. Since both sets $h H_{z_{0}}^{\prime}$ and $g H_{z_{0}}^{(k)}$ are irreducible, analytic sets in $G$ of the same dimension, we have $h H_{z_{0}}^{\prime}=g H_{z_{0}}^{(k)}$. Thus the reverse inclusion holds, and the second equality is proved.

Proposition 6.5. The pair $\left(\widehat{\pi}, M^{\prime}\right)$ gives a normal, unramified cover of $M$; i.e., if $z \in M, w \in \widehat{\pi}^{-1}(z)$, and $\gamma$ is a real, one-dimensional curve in $M$ starting at $z$, then there exists a unique curve $\gamma^{\prime}$ in $M^{\prime}$ which starts at $w$ and satisfies $\widehat{\pi}\left(\gamma^{\prime}\right)=\gamma$.

Proof. We use the notation in Proposition 6.4, in particular, $v_{0}$ is a neighborhood of $e$ in $G$ satisfying the conditions 1 and 2. Let $z \in M$; thus $z=g H_{z_{0}}$ for some $g \in G$, or equivalently $z=g\left(z_{0}\right)$. Then we have by (6.30)

$$
\widehat{\pi}^{-1}(z)=\left\{w_{k}\right\}_{k=1,2, \ldots} \quad \text { where } w_{k}=g H_{z_{0}}^{(k)}=g h_{k} H_{z_{0}}^{\prime} \in M^{\prime} .
$$

Note that $\left\{w_{k}\right\}_{k=1,2, \ldots}$ are isolated in $M^{\prime}$. We define

$$
V:=g v_{0} H_{z_{0}} \quad \text { and } \quad U_{k}:=g v_{0} H_{z_{0}}^{(k)}=g v_{0} h_{k} \widehat{H}_{w_{0}}=g v_{0} \widehat{H}_{w_{0}} h_{k},
$$

so that $V$ is a neighborhood of $z$ in $M$ and $U_{k}$ is a neighborhood of $w_{k}$ in $M^{\prime}$. It suffices to prove that

$$
\begin{aligned}
& \widehat{\pi}^{-1}(V)=\bigcup_{k=1}^{\infty} U_{k} \text { a disjoint union; } \\
& \widehat{\pi}: U_{k} \mapsto V \text { is bijective for each } k=1,2, \ldots
\end{aligned}
$$

The first equality, and the disjointness, follow from 1. in Proposition 6.4. The surjectivity of $\widehat{\pi}$ in the second assertion is clear by its definition. To verify injectivity, it suffices to show that $g a H_{z_{0}}=g b H_{z_{0}} \Longrightarrow g a H_{z_{0}}^{(k)}=g b H_{z_{0}}^{(k)}$ for $a, b \in v_{0}$, or, equivalently, since $H_{z_{0}}^{(k)}=h_{k} H_{z_{0}}^{\prime}, a^{-1} b \in H_{z_{0}} \Longrightarrow h_{k}^{-1}\left(a^{-1} b\right) h_{k} \in$ $H_{z_{0}}^{\prime}$. Since $H_{z_{0}}^{\prime}$ is a normal subgroup of $H_{z_{0}}$, this last implication follows from 2. in Proposition 6.4.

We set up some more notation which will be useful in discussing the case when $H_{z_{0}}$ is not connected. For our relatively compact domain $D$ in $M$, we take $z_{0} \in D$ and set $\widehat{D}:=\widehat{\pi}^{-1}(D)$ in $M^{\prime}$. Note that $\widehat{D}$ need not be relatively compact in $M^{\prime}$; moreover, $\widehat{D}$ may be disconnected. However, from 
Proposition 6.5, $\widehat{D}$ has no relative boundary over $D$; and from (6.33), for $w \in$ $\partial \widehat{D}$ we have $\widehat{\pi}(w) \in \partial D$. We decompose $\widehat{D}$ into its connected components $\widehat{D}_{j}$ in $M^{\prime}: \widehat{D}=\bigcup_{j=1}^{\infty} \widehat{D}_{j}$. We use the convention that $\widehat{D}_{1}$ is the connected component containing $w_{0}$. Recalling the notation $D^{\prime}\left(z_{0}\right), D^{(i)}\left(z_{0}\right)$ in (6.4) for the connected components of $D\left(z_{0}\right)$ in $G$, we will show in Appendix B that we have, after possibly relabeling indices,

$$
\widehat{D}_{k}=\left\{g\left(w_{0}\right) \in M^{\prime}: g \in D^{(k)}\left(z_{0}\right)\right\}, \quad k=1,2, \ldots
$$

We introduce the notation

$$
\widehat{\lambda}(w):=\lambda(\widehat{\pi}(w)), \quad w \in \widehat{D}_{1} .
$$

Note that this notation implies that $\widehat{\lambda}(w)$ is related to $\widehat{\pi}: M^{\prime}=G / \widehat{H}_{w_{0}} \mapsto$ $M=G / H_{z_{0}}$, but we caution the reader that $\widehat{\lambda}(w)$ is not necessarily the $c$ Robin function for $\widehat{D}_{1}$ in $M^{\prime}$. However, since $\widehat{D}_{1}$ is an unramified cover over $D$ without relative boundary, it follows that $-\widehat{\lambda}(w)$ is, indeed, a plurisubharmonic function on the domain $\widehat{D}_{1} \subset M^{\prime}$ such that for $w^{\prime} \in \partial \widehat{D}_{1}$ we have $\lim _{w \rightarrow w^{\prime}} \widehat{\lambda}(w)=-\infty$. Moreover, if $K \Subset D$ and we set $\widehat{K}_{1}:=\widehat{\pi}^{-1}(K) \cap \widehat{D}_{1}$, then $\widehat{\lambda}(w)$ is bounded in $\widehat{K}_{1}$.

We define

$$
\widehat{\mathfrak{X}}_{w_{0}}:=\left\{X \in \mathfrak{X}:\left.\left[\frac{\partial^{2} \widehat{\lambda}\left(\exp t X\left(w_{0}\right)\right)}{\partial t \partial \bar{t}}\right]\right|_{t=0}=0\right\} .
$$

With the notation above, we will show in Appendix B that

$$
\widehat{\mathfrak{X}}_{w_{0}}=\mathfrak{X}_{z_{0}}=\left\{X \in \mathfrak{X}: \exp t X\left(w_{0}\right) \in \widehat{D}_{1}, \widehat{\lambda}\left(\exp t X\left(w_{0}\right)\right)=\widehat{\lambda}\left(w_{0}\right), t \in \mathbb{C}\right\}
$$

and for $X \in \widehat{\mathfrak{X}}_{w_{0}}$ and $g \in \widehat{D}_{1}\left(w_{0}\right)$,

$$
g \exp t X\left(w_{0}\right) \in \widehat{D}_{1}, \quad \widehat{\lambda}\left(g \exp t X\left(w_{0}\right)\right)=\widehat{\lambda}\left(g\left(w_{0}\right)\right)
$$

for all $t \in \mathbb{C}$. Associated to $\widehat{\mathfrak{X}}_{w_{0}}$ is the Lie subgroup

$$
\widehat{\Sigma}_{w_{0}}=\left\{\prod_{i=1}^{\nu} \exp t_{i} X_{i} \in G: \nu \in \boldsymbol{Z}^{+}, t_{i} \in \mathbb{C}, X_{i} \in \widehat{\mathfrak{X}}_{w_{0}}\right\} .
$$

We now state the main theorem in case $H_{z}$ is not connected, and then we make a remark on the roles of the Lie subalgebra $\widehat{\mathfrak{X}}_{w_{0}}$ of $\mathfrak{X}$ and Lie subgroup $\widehat{\Sigma}_{w_{0}}$. In assertion (o) in $2-a$. in the theorem below, $\mathcal{H}^{\prime}\left(z_{0}\right)$ is the Lie subgroup of $H_{z_{0}}$ studied in Corollary 6.1; $\Sigma_{z_{0}}$ is the connected Lie subgroup of $G$ corresponding to the Lie subalgebra $\mathfrak{X}_{0}$ defined in (6.11), and

$$
\Sigma_{z_{0}} \mathcal{H}^{\prime}\left(z_{0}\right)=\left\{s h \in G: s \in \Sigma_{z_{0}}, h \in \mathcal{H}^{\prime}\left(z_{0}\right)\right\} .
$$

As mentioned, the proof of the theorem will be deferred to Appendix B. 
Theorem 6.4. Under the same notation as in Theorem 6.3; i.e., $M$ is a homogeneous space of dimension $n$ and the Lie transformation group $G$ of dimension $m$ acts transitively on $M$, assume that the isotropy subgroup $H_{z}$ of $G$ for $z \in M$ is not connected. Let $D$ be a pseudoconvex domain in $M$ with smooth boundary. Assume that $D$ is not Stein. Fix $z_{0} \in D$. Then:

2-a. The subset $\sigma_{z_{0}}=\Sigma_{z_{0}}\left(z_{0}\right)$ of $M$ defined in (6.16) is a parabolic $m_{1}$ dimensional non-singular $f$-generalized analytic set in $M$ passing through $z_{0}$ with the following properties:

(o) $\sigma_{z_{0}} \Subset D ; \Sigma_{z_{0}} \mathcal{H}^{\prime}\left(z_{0}\right)=D^{\prime}\left(z_{0}\right) \cap$ Aut $\sigma_{z_{0}} ; \Sigma_{z_{0}} \mathcal{H}^{\prime}\left(z_{0}\right)$ is a Lie subgroup of $G$ which acts transitively on $\sigma_{z_{0}}$; and

$$
\sigma_{z_{0}} \approx \Sigma_{z_{0}} \mathcal{H}^{\prime}\left(z_{0}\right) / \mathcal{H}^{\prime}\left(z_{0}\right)
$$

(i) There exists a domain $K$ with $D \Subset K \subset M$ such that $D(K)$ is foliated by the sets $g \sigma_{z_{0}}, g \in K^{\prime}\left(z_{0}\right)\left(g \in D^{\prime}\left(z_{0}\right)\right)$ and each such set $g \sigma_{z_{0}}$ is relatively compact in $K(D)$ :

$$
K=\bigcup_{g \in K^{\prime}\left(z_{0}\right)} g \sigma_{z_{0}} \quad \text { and } \quad D=\bigcup_{g \in D^{\prime}\left(z_{0}\right)} g \sigma_{z_{0}} .
$$

(ii) The same result as (ii) in 1-a. of Theorem 6.3 holds.

2-b. Assume that $\sigma_{z_{0}}$ is closed in $M$. Then

(o) The same result as (o) in 1-a. of Theorem 6.3 holds.

(i) There exists a complex manifold $K_{0}$ and a holomorphic map $\pi_{0}$ : $K \mapsto K_{0}$ such that $\pi_{0}^{-1}(\zeta) \approx \sigma_{z_{0}}$ (as complex manifolds) for each $\zeta \in K_{0}$; moreover, there exists a Stein domain $D_{0} \Subset K_{0}$ with smooth boundary such that $D=\pi_{0}^{-1}\left(D_{0}\right)$.

Remark 6.1. We first remark that, as in the connected case, we have

$$
\mathfrak{h}_{z_{0}} \subsetneq \mathfrak{X}_{z_{0}} \subsetneq \mathfrak{X}
$$

where $\mathfrak{h}_{z_{0}}$ is the Lie subalgebra of $\mathfrak{X}$ corresponding to $H_{z_{0}}^{\prime}$ and $\mathfrak{X}_{z_{0}}=\widehat{\mathfrak{X}}_{w_{0}}$ from (6.35). Also, in the proof of Theorem 6.4 the connected Lie subalgebra $\mathfrak{X}_{z_{0}}=\widehat{\mathfrak{X}}_{w_{0}}$ of $\mathfrak{X}$ defined by (6.35) and the connected Lie subgroup $\Sigma_{z_{0}}=\widehat{\Sigma}_{w_{0}}$ of $G$ which corresponds to $\mathfrak{X}_{z_{0}}$ will play essential roles. In the special cases when $H_{z_{0}} \subset \Sigma_{z_{0}}$ and $\Sigma_{z_{0}}$ is closed in $G$, we will see from the proof that $2-b$. in Theorem 6.4 implies that $K=M ; K_{0}=G / \Sigma_{z_{0}}$ (hence $K_{0}$ is a homogeneous space); $\pi_{0}: g H_{0} \in M \mapsto g \Sigma_{z_{0}} \in K_{0}$; and $D_{0}$ is a Stein domain in $K_{0}$ with smooth boundary. That is, in this case, the result is the same as in $1-b$. of Theorem 6.3. 
This special case described in Remark6.1 occurs when $M$ is a special Hopf manifold; we now turn our attention to this case. We let $\left(\mathbb{C}^{n}\right)^{*}:=\mathbb{C}^{n} \backslash\{0\}$ and we fix $\alpha \in \mathbb{C}$ with $|\alpha| \neq 1$. For $z, z^{\prime} \in\left(\mathbb{C}^{n}\right)^{*}$, we define the following equivalence relation in $\left(\mathbb{C}^{n}\right)^{*}$ :

$$
z \sim w \quad \text { iff } \quad w=\alpha^{k} z \text { for some integer } k .
$$

We consider the following space:

$$
\mathbb{H}_{n}=\left(\mathbb{C}^{n}\right)^{*} / \sim
$$

this is an $n$-dimensional compact manifold. The space $\mathbb{H}_{n}$ is called a special Hopf manifold. We write $[z] \in \mathbb{H}_{n}$ to denote the equivalence class of a point $z=\left(z_{1}, \ldots, z_{n}\right) \in\left(\mathbb{C}^{n}\right)^{*}$. In case $n=1, \mathbb{H}_{1}$ is the usual one-dimensional complex torus $T_{\alpha}$. The space $\mathbb{H}_{n}$ clearly has the following property.

\section{Proposition 6.6.}

1. The group $G L(n, \mathbb{C})$ is a Lie transformation group of $\mathbb{H}_{n}$ and acts transitively on $\mathbb{H}_{n}$; i.e., $\mathbb{H}_{n}$, equipped with the Lie group $G L(n, \mathbb{C})$, is a homogeneous space.

2. We have the canonical projection $\pi_{0}:\left[z_{1}, \ldots, z_{n}\right] \in \mathbb{H}_{n} \mapsto\left[z_{1}: \ldots\right.$ : $\left.z_{n}\right] \in \mathbb{P}^{n-1}$ such that $\pi_{0}^{-1}(\zeta) \approx T_{\alpha}$ for each $\zeta \in \mathbb{P}^{n-1}$.

We write $O:=[(1,0, \ldots, 0)] \in \mathbb{H}_{n}$ and call $O$ the base point of $\mathbb{H}_{n}$. Then the isotropy subgroup $H_{0}$ of $G L(n, \mathbb{C})$ for $O$ is

$$
H_{0}=\left\{\left(\begin{array}{c|c}
\alpha^{k} & (*) \\
\hline 0 & \\
\vdots & A \\
0 &
\end{array}\right) \in G L(n, \mathbb{C}): k \in Z,(*) \in \mathbb{C}^{n-1}, \operatorname{det} A \neq 0\right\},
$$

which is closed but not connnected in $G L(n, \mathbb{C})$. We let $H_{0}^{\prime}$ denote the connected component of $H_{0}$ which contains the identity $I_{n}$ in $G L(n, \mathbb{C})$, i.e.,

$$
H_{0}^{\prime}=\left\{\left(\begin{array}{c|c}
1 & (*) \\
\hline 0 & \\
\vdots & A \\
0 &
\end{array}\right) \in G L(n, \mathbb{C}):(*) \in \mathbb{C}^{n-1}, \operatorname{det} A \neq 0\right\} .
$$

Therefore,

$$
\mathbb{H}_{n} \approx G L(n, \mathbb{C}) / H_{0}=\left\{g H_{0}: g \in G L(n, \mathbb{C})\right\}
$$


To be precise, let

$$
g=\left(\begin{array}{ccc}
g_{11} & \cdots & g_{1 n} \\
\vdots & \ddots & \vdots \\
g_{n 1} & \cdots & g_{n n}
\end{array}\right) \in G L(n, \mathbb{C})
$$

Let $\mathbf{g}:=\left(g_{11}, \ldots, g_{n 1}\right) \in\left(\mathbb{C}^{n}\right)^{*}$ denote the first column vector of $g$. Then the mapping

$$
\alpha_{0}: g H_{0} \in G L(n, \mathbb{C}) / H_{0} \rightarrow g(O)=[\mathbf{g}] \in \mathbb{H}_{n}
$$

is bijective.

For local coordinates in a neighborhood $V$ of the base point $O$ we can take

$$
\left(\begin{array}{cccc}
1+t_{1} & & & \\
t_{2} & 1 & & \\
\vdots & & \ddots & \\
t_{n} & & & 1
\end{array}\right), \quad\left|t_{i}\right|<\rho, i=1, \ldots, n
$$

where the missing entries are all 0 . Equivalently, let $U:=\left\{\mathbf{t}=\left(t_{1}, \ldots, t_{n}\right) \in\right.$ $\left.\mathbb{C}^{n}:\left|t_{i}\right|<\rho\right\}$ where the base point $O$ of $\mathbb{H}_{n}$ corresponds to the origin 0 of $\mathbb{C}^{n}$. That is, let $g \in G L(n, \mathbb{C})$ in (6.39) be close to the identity $I_{n}$. Corresponding to $g H_{0} \in \mathbb{H}_{n}$ the point $\mathbf{t}(g):=\left(g_{11}-1, g_{21}, \ldots, g_{n 1}\right) \in \mathbb{C}^{n}$, is close to $(0,0, \ldots, 0)$. We have (i) if $g_{1} H_{0} \neq g_{2} H_{0}$ for $g_{1}, g_{2} \in V$, then $\mathbf{t}\left(g_{1}\right) \neq \mathbf{t}\left(g_{2}\right)$; (ii) given $\mathbf{t}^{\prime} \in U$, we can find $g \in G L(n, \mathbb{C})$ close to $I_{n}$ with $\mathbf{t}(g)=\mathbf{t}^{\prime}$. We call the local coordinates $\mathbf{t}$ at $O$ the standard local coordinates at $O$ in $\mathbb{H}_{n}$.

We consider the Lie algebra $\mathfrak{X}$ consisting of all left-invariant holomorphic vector fields $X$ on $G L(n, \mathbb{C})$. We identify $\mathfrak{X}$ with $M_{n}(\mathbb{C})$, the set of all $n \times n$ square matrices, as follows: to $X=\left(\lambda_{i j}\right) \in M_{n}(\mathbb{C})$ we associate a left-invariant holomorphic vector field $v_{X}$ on $G L(n, \mathbb{C})$ via, for $g=\left(x_{i j}\right) \in$ $G L(n, \mathbb{C})$

$$
v_{X}(g):=\sum_{i, j=1}^{n} \lambda_{i j} X_{i j}(g), \quad \text { where } \quad X_{i j}(g)=\sum_{k=1}^{n} x_{k i} \frac{\partial}{\partial x_{k j}} .
$$

Hence we identify the vector field $v_{X}$ on $G L(n, \mathbb{C})$ with the matrix $X=\left(\lambda_{i j}\right)$ in $M_{n}(\mathbb{C})$ as additive groups. The integral curve $C_{X}$ for $v_{X}$ with initial value $I_{n}$ is given by

$$
C_{X}=\{\exp t X \in G L(n, \mathbb{C}): t \in \mathbb{C}\},
$$

and the integral curve of $v_{X}$ with initial value $g \in G L(n, \mathbb{C})$ is given by $g C_{X} \in G L(n, \mathbb{C})$. 
We let $\mathfrak{h}_{0}$ denote the corresponding Lie subalgebra for $H_{0}^{\prime}$, so that

$$
\mathfrak{h}_{0}=\left\{\left(\begin{array}{cc}
0 & \\
\vdots & A \\
0 &
\end{array}\right) \in M_{n}(\mathbb{C}): A \in M_{n, n-1}(\mathbb{C})\right\}
$$

where $M_{n, n-1}(\mathbb{C})$ denotes the set of all $n \times(n-1)$-matrices. We have

$$
H_{0}^{\prime}=\left\{\prod_{i=1}^{\nu} \exp t_{i} X_{i} \in G L(n, \mathbb{C}): \nu \in Z^{+}, t_{i} \in \mathbb{C}, X_{i} \in \mathfrak{h}_{0}\right\} .
$$

Theorem 6.5. Let $D \Subset \mathbb{H}_{n}$ be a pseudoconvex domain with smooth boundary which is not Stein. Then there exists a Stein domain $D_{0}$ in $\mathbb{P}^{n-1}$ with smooth boundary such that $D=\pi_{0}^{-1}\left(D_{0}\right)$.

Proof. We may assume $D$ contains the base point $O$. Following Theorem 6.4 for such domains $D$ in a homogeneous space, we fix a Kähler metric $d s^{2}$ on $G=G L(n, \mathbb{C})$ and a strictly positive $C^{\infty}$ function $c$ on $G$ and we consider the $c$-Robin function $\lambda([z])$ for $D$. Define the following subset of the Lie algebra $\mathfrak{X}=M_{n}(\mathbb{C})$ :

$$
\mathfrak{X}_{0}=\left\{X \in \mathfrak{X}:\left.\left[\frac{\partial^{2} \lambda(\exp t X(O))}{\partial t \partial \bar{t}}\right]\right|_{t=0}=0\right\} .
$$

Under our assumptions for $D$ we showed that $\mathfrak{X}_{0}$ is a Lie subalgebra of $\mathfrak{X}$ with

$$
\mathfrak{h}_{0} \varsubsetneqq \mathfrak{X}_{0} \varsubsetneqq \mathfrak{X}
$$

(recall (6.12) and Remark 6.1). Fix $X \in \mathfrak{X}_{0} \backslash \mathfrak{h}_{0}$. We may assume that $X$ is of the form

$$
X=\left(\begin{array}{cc}
c_{1} & \\
\vdots & (*) \\
c_{n} &
\end{array}\right) \in M_{n}(\mathbb{C})
$$

where some $c_{i} \neq 0, i=1, \ldots, n$, and $(*)$ is a matrix in $M_{n, n-1}(\mathbb{C})$. We show that

$$
c_{j}=0, \quad j=2, \ldots, n
$$

We verify (6.43) by contradiction. Thus we assume that some $c_{j} \neq 0, j=$ $2, \ldots, n$. Take any $Y \in \mathfrak{h}_{0}$ of the form $Y=(\mathbf{0}, \ldots, \mathbf{0}, \mathbf{y}, \mathbf{0}, \ldots, \mathbf{0})$, where $\mathbf{0}$ 
is the zero $n$-colmun vector and $\mathbf{y}$ is an $n$-column vector in the $j$-th column. An elementary calculation yields

$$
[X, Y]=\left(\begin{array}{cc}
-y_{1} c_{j} & \\
\vdots & (0) \\
-y_{n} c_{j} &
\end{array}\right)+Y^{\prime}
$$

where $(0)$ is the zero matrix in $M_{n, n-1}(\mathbb{C})$ and $Y^{\prime} \in \mathfrak{h}_{0}$. Recall that $\mathfrak{X}_{0}$ is a Lie subalgebra of $\mathfrak{X}$ with $\mathfrak{h}_{0} \subset \mathfrak{X}_{0}$. Since the points $y_{j}, j=1, \ldots, n$, can be arbitrarily chosen in $\mathbb{C}$, if $c_{j} \neq 0$ it follows that $\mathfrak{X}_{0}=\mathfrak{X}$. This contradicts (6.42), which proves (6.43).

We thus see that if $\mathfrak{X}_{0} \neq \mathfrak{h}_{0}$, then, $\operatorname{dim} \mathfrak{X}_{0}=1+n(n-1)$ and

$$
\mathfrak{X}_{0}=\left\{\left(\begin{array}{cc}
x & \\
0 & \\
\vdots & (*) \\
0 &
\end{array}\right): x \in \mathbb{C},(*) \in M_{n, n-1}(\mathbb{C})\right\}
$$

To complete the proof of the theorem we let $\Sigma_{0}$ denote the integral manifold for $\mathfrak{X}_{0}$ in $G L(n, \mathbb{C})$ :

$$
\Sigma_{0}=\left\{\prod_{i=1}^{\nu} \exp t_{i} X_{i} \in G L(n, \mathbb{C}): \nu \in \boldsymbol{Z}^{+}, t_{i} \in \mathbb{C}, \quad X_{i} \in \mathfrak{X}_{0}\right\},
$$

so that

$$
\Sigma_{0}=\left\{\left(\begin{array}{c|c}
a & (*) \\
\hline 0 & \\
\vdots & A \\
0 &
\end{array}\right): a \in \mathbb{C}^{*} ;(*) \in \mathbb{C}^{n-1}, A \in G L(n-1, \mathbb{C})\right\} .
$$

Hence $\Sigma_{0}$ is a closed Lie subgroup of $G L(n, \mathbb{C})$ with $H_{0} \subset \Sigma_{0}$. Moreover, writing ${ }^{t} O$ for the transpose of the row vector $O=[(1,0, \ldots, 0)]$,

$$
\sigma_{0}:=\psi_{0}\left(\Sigma_{0}\right)=\Sigma_{0}\left({ }^{t} O\right)=\left\{[(a, 0, \ldots, 0)] \in \mathbb{H}_{n}: a \in \mathbb{C}^{*}\right\},
$$

which coincides with the torus $T_{\alpha}$. Since $G L(n, \mathbb{C}) / \Sigma_{0}=\mathbb{P}^{n-1}$ and the projection $g H_{0} \mapsto g \Sigma_{z_{0}}$ from $\mathbb{H}_{n}$ to $\mathbb{P}^{n-1}$ mentioned in Remark 6.1 coincides with $\pi_{0}$ in 2. of Proposition 6.6. Theorem 6.5 follows from Remark 6.1 (taking $K=\mathbb{H}_{n}$ and $\left.K_{0}=\mathbb{P}^{n-1}\right)$. 


\section{$7 \quad$ Flag space}

In this section, we apply $1-b$. in Theorem 6.3 to the flag space $\mathcal{F}_{n}$ to determine all pseudoconvex domains $D \Subset \mathcal{F}_{n}$ with smooth boundary which are not Stein (see Theorem 17.1). There are few known results on the Levi problem in $\mathcal{F}_{n}$ (cf., Y-T. Siu [14], K. Adachi [1]). By definition, the flag space $\mathcal{F}_{n}$ is the set of all nested sequences

$$
z:\{0\} \subset F_{1} \subset \ldots \subset F_{n-1} \subset \mathbb{C}^{n}
$$

where $F_{i}, i=1, \ldots, n-1$ is an $i$-dimensional vector subspace of $\mathbb{C}^{n}$. We describe the structure of $\mathcal{F}_{n}$ as a homogeneous space. Given $A=\left(a_{i j}\right) \in$ $G L(n, \mathbb{C})$ we shall define an isomorphism $\mathcal{A}$ of $\mathcal{F}_{n}$. Consider the linear transformation of $\mathbb{C}^{n}$ given by

$$
A:\left(\begin{array}{c}
Z_{1} \\
\vdots \\
Z_{n}
\end{array}\right)=\left(\begin{array}{ccc}
a_{11} & \cdots & a_{1 n} \\
\vdots & \ddots & \vdots \\
a_{n 1} & \cdots & a_{n n}
\end{array}\right)\left(\begin{array}{c}
z_{1} \\
\vdots \\
z_{n}
\end{array}\right) .
$$

For $z \in \mathcal{F}_{n}$ as in (7.1), we then define $\mathcal{A}(z) \in \mathcal{F}_{n}$ via

$$
\mathcal{A}(z): \quad\{0\} \subset A\left(F_{1}\right) \subset A\left(F_{2}\right) \subset A\left(F_{n-1}\right) \subset \mathbb{C}^{n} .
$$

In this way $G L(n, \mathbb{C})$ acts transitively on $\mathcal{F}_{n}$; i.e., $\mathcal{F}_{n}$ is a homogeneous space with Lie transformation group $G L(n, \mathbb{C})$.

We fix the following point $O$ in $\mathcal{F}_{n}$ :

$$
O: \quad\{0\} \subset F_{1}^{0} \subset F_{2}^{0} \subset \cdots \subset F_{n-1}^{0} \subset \mathbb{C}^{n},
$$

where

$$
F_{i}^{0}: z_{i+1}=\cdots=z_{n}=0, \quad i=1, \ldots, n-1 .
$$

We call $O$ the base point of $\mathcal{F}_{n}$. The isotropy subgroup $H_{0}$ of $G L(n, \mathbb{C})$ for the point $O$ is the set of all upper triangular non-singular matrices:

$$
H_{0}=\left\{\left(\begin{array}{cccc}
a_{11} & a_{12} & \cdots & a_{1 n} \\
0 & a_{22} & \cdots & a_{2 n} \\
& \ddots & \ddots & \\
0 & 0 & 0 & a_{n n}
\end{array}\right) \in G L(n, \mathbb{C})\right\} .
$$

In particular, $H_{0}$ is connected in $G L(n, \mathbb{C})$ and $\operatorname{dim} H_{0}=\frac{n(n+1)}{2}$. Since $\mathcal{F}_{n} \approx G L(n, \mathbb{C}) / H_{0}$, the flag space $\mathcal{F}_{n}$ is a compact manifold with $\operatorname{dim} \mathcal{F}_{n}=$ $N:=\frac{n(n-1)}{2}$. 
For local coordinates of a neighborhood of the base point $O$ in $\mathcal{F}_{n}$ we can take

$$
\left(\begin{array}{cccc}
1 & & & \\
& \ddots & 0 & \\
& t_{i j} & \ddots & \\
& & & 1
\end{array}\right), \quad t_{i j} \in \mathbb{C}, \quad 1 \leq j<i \leq n-1,
$$

where the point $O$ corresponds to the identity $I$ in $G L(n, \mathbb{C})$. Equivalently

$$
\begin{aligned}
\mathbf{t} & =\left(t_{21}, t_{31}, \ldots, t_{n 1} ; t_{32}, \ldots, t_{n 2} ; \ldots ; t_{n n-1}\right) \\
& \equiv\left(\mathbf{t}_{1} ; \mathbf{t}_{2} ; \ldots ; \mathbf{t}_{n-1}\right) \in \mathbb{C}^{N}
\end{aligned}
$$

where $\mathbf{t}_{j}, j=1, \ldots, n-1$ is an $(n-j)$-column vector and the base point $O$ corresponds to the origin $\mathbf{0}$ in $\mathbb{C}^{N}$. We call these local coordinates the standard local coordinates at $O$.

For any $A=\left(a_{i j}\right) \in G L(n, \mathbb{C})$ which is sufficiently close to $I$, each subspace $A\left(F_{k}^{0}\right), k=1, \ldots, n-1$, in (7.2) can be written as

$$
A\left(F_{k}^{0}\right):\left\{\begin{array}{ccc}
Z_{k+1} & =\alpha_{k+11}^{(k)} Z_{1}+\cdots & +\alpha_{k+1 k}^{(k)} Z_{k} \\
\vdots & \vdots & \vdots \\
Z_{n} & =\alpha_{n 1}^{(k)} Z_{1}+\cdots & +\alpha_{n k}^{(k)} Z_{k}
\end{array}\right.
$$

where

$$
\begin{aligned}
\left(\begin{array}{ccc}
\alpha_{k+11}^{(k)} & \cdots & \alpha_{k+1 k}^{(k)} \\
\vdots & \ddots & \vdots \\
\alpha_{n 1}^{(k)} & \cdots & \alpha_{n k}^{(k)}
\end{array}\right) & =\left(\begin{array}{ccc}
a_{k+11} & \ldots & a_{k+1 k} \\
\vdots & \ddots & \vdots \\
a_{n 1} & \ldots & a_{n k}
\end{array}\right)\left(\begin{array}{ccc}
a_{11} & \cdots & a_{1 k} \\
\vdots & \ddots & \vdots \\
a_{k 1} & \cdots & a_{k k}
\end{array}\right)^{-1} \\
& \equiv A_{n-k}^{k} A_{k}^{-1} .
\end{aligned}
$$

We call (7.3) the canonical representation of the $k$-dimensional vector space $A\left(F_{k}^{0}\right)$. Using the standard local coordinates $\mathbf{t}$, we let

$$
\gamma:=\left(\gamma_{21}, \ldots, \gamma_{n 1} ; \gamma_{32}, \ldots, \gamma_{n 2} ; \ldots ; \gamma_{n n-1}\right)
$$

denote the point $\mathcal{A}(O)$ in $\mathcal{F}_{n}$. Then

$$
\left(\begin{array}{c}
\gamma_{k+1 k} \\
\vdots \\
\gamma_{n k}
\end{array}\right)=\left(\begin{array}{c}
\alpha_{k+1 k}^{(k)} \\
\vdots \\
\alpha_{n k}^{(k)}
\end{array}\right), \quad k=1, \ldots, n-1,
$$

which is equal to the $k$-column vector determined by the coefficients of the variable $Z_{k}$ in the canonical representation (7.3) of $A\left(F_{k}^{0}\right)$. 
Recall that $M_{n}(\mathbb{C})$ denotes the set of all $n \times n$-matrices with coefficients in $\mathbb{C}$ and we associate $X=\left(\lambda_{i j}\right) \in M_{n}(\mathbb{C})$ to a left-invariant holomorphic vector field $v_{X}$ on $G L(n, \mathbb{C})$ as in (6.40): for $g=\left(x_{i j}\right) \in G L(n, \mathbb{C})$,

$$
v_{X}(g):=\sum_{i, j=1}^{n} \lambda_{i j} X_{i j}(g), \quad \text { where } \quad X_{i j}(g)=\sum_{k=1}^{n} x_{k i} \frac{\partial}{\partial x_{k j}} .
$$

Then $C_{X}=\{\exp t X \in G L(n, \mathbb{C}): t \in \mathbb{C}\}$ is the integral curve of $v_{X}$ with initial value $I$ and $A C_{X} \in G L(n, \mathbb{C})$ is the integral curve of $v_{X}$ with initial value $A \in G L(n, \mathbb{C})$.

This identification of left-invariant holomorphic vector fields and matrices will be heavily utilized. Also, unless otherwise stated, in the matrices occurring in this section all missing entries are 0 .

\section{Lemma 7.1 .}

1. Let $X=\left(\lambda_{i j}\right) \in M_{n}(\mathbb{C})$ and let $C_{X}(O):=\left\{(\exp t X)(O) \in \mathcal{F}_{n}: t \in \mathbb{C}\right\}$ be an analytic curve passing through the base point $O$ at $t=0$. Then the tangent vector of $C_{X}(O)$ at $O$, in terms of the standard local coordinates t at $O$, has the form

$$
\left(\lambda_{21}, \lambda_{31}, \ldots, \lambda_{n 1} ; \lambda_{32}, \ldots, \lambda_{n 2} ; \ldots ; \lambda_{n n-1}\right) .
$$

2. Let $X=\left(\lambda_{i j}\right) \in M_{n}(\mathbb{C})$ and let $X^{\prime} \in M_{n}(\mathbb{C})$ be the matrix

$$
X^{\prime}:=\left(\begin{array}{lllll}
0 & & & & \\
\lambda_{21} & 0 & & & \\
\lambda_{31} & \lambda_{32} & 0 & & \\
\vdots & \vdots & \ddots & \ddots & \\
\lambda_{n 1} & \lambda_{n 2} & \cdots & \lambda_{n n-1} & 0
\end{array}\right)
$$

For any $A \in H_{0}$, the direction of the analytic curve $A \exp t X(O)$ at $O$ in $\mathcal{F}_{n}$ is equal to that of the curve $A \exp t X^{\prime}(O)$.

Proof. We have

$$
\exp t X=\left(\begin{array}{ccc}
1+\lambda_{11} t & \cdots & \lambda_{1 n} t \\
\vdots & \ddots & \vdots \\
\lambda_{n 1} t & \cdots & 1+\lambda_{n n} t
\end{array}\right)+O\left(t^{2}\right) .
$$

In terms of the standard local coordinates at $O$, we write

$$
\mathbf{r}(t):=\left(\mathbf{r}_{1}(t) ; \mathbf{r}_{2}(t) ; \ldots ; \mathbf{r}_{n-1}(t)\right)
$$


for the point $\exp t X(O)$ in $\mathcal{F}_{n}$. Fix $1 \leq k \leq n-1$. Following (7.4) we consider the $(n-k, k)$-matrix $A_{n-k}^{k}(t)$ and $(k, k)$-matrix $A_{k}(t)^{-1}$ for $A(t)=\exp t X$ :

$$
\begin{aligned}
A_{n-k}^{k}(t) & =\left(\begin{array}{ccc}
\lambda_{k+11} t & \cdots & \lambda_{k+1 k} t \\
\vdots & \ddots & \vdots \\
\lambda_{n 1} t & \cdots & \lambda_{n k} t
\end{array}\right), \\
A_{k}(t)^{-1} & =\left(\begin{array}{ccc}
1+\lambda_{11} t & \cdots & \lambda_{1 k} t \\
\vdots & \ddots & \vdots \\
\lambda_{k 1} t & \cdots & 1+\lambda_{k k} t
\end{array}\right)^{-1} \\
& \equiv\left(\mathbf{d}_{1}^{(k)}(t), \ldots, \mathbf{d}_{k}^{(k)}(t)\right),
\end{aligned}
$$

where each $\mathbf{d}_{j}^{(k)}(t) j=1, \ldots, k$ is a $k$-column vector. We then have

$$
{ }^{t} \mathbf{r}_{k}(t)=A_{n-k}^{k}(t) \mathbf{d}_{k}^{(k)}(t)+O\left(t^{2}\right)
$$

so that

$$
\left.\left[\frac{d}{d t}{ }^{t} \mathbf{r}_{k}(t)\right]\right|_{t=0}=\left(A_{n-k}^{k}\right)^{\prime}(0) \mathbf{d}_{k}^{(k)}(0)=\left(A_{n-k}^{k}\right)^{\prime}(0)\left(\begin{array}{c}
0 \\
\vdots \\
0 \\
1
\end{array}\right)=\left(\begin{array}{c}
\lambda_{k+1 k} \\
\vdots \\
\lambda_{n-1 k} \\
\lambda_{n k}
\end{array}\right)
$$

which proves 1 .

To prove 2. we write $X=\left(\lambda_{i j}\right) \in M_{n}(\mathbb{C})$ and $A=\left(a_{i j}\right) \in H_{0}$. We denote by $\mathbf{v}:=\left(\mathbf{v}_{1} ; \mathbf{v}_{2} ; \cdots ; \mathbf{v}_{n-1}\right)$ and $\mathbf{v}^{\prime}:=\left(\mathbf{v}_{1}^{\prime} ; \mathbf{v}_{2}^{\prime} ; \cdots ; \mathbf{v}_{n-1}^{\prime}\right)$ the directions of the curves $A \exp t X(O)$ and $A \exp t X^{\prime}(O)$ at $O$ in $\mathcal{F}_{n}$. Following (7.4), for $k=1, \ldots, n-1$ we have

$$
{ }^{t} \mathbf{v}_{k}=\left(\begin{array}{ccc}
\sum_{i=k+1}^{n} a_{k+1 i} \lambda_{i 1}, & \ldots, & \sum_{i=k+1}^{n} a_{k+1 i} \lambda_{i k} \\
\sum_{i=k+2}^{n} a_{k+2 i} \lambda_{i 1}, & \ldots, & \sum_{i=k+2}^{n} a_{k+2 i} \lambda_{i k} \\
\vdots & \ddots & \vdots \\
a_{n n} \lambda_{n 1}, & \ldots & a_{n n} \lambda_{n k}
\end{array}\right)\left(\begin{array}{c}
a_{1 k}^{(k)} \\
a_{2 k}^{(k)} \\
\vdots \\
a_{k k}^{(k)}
\end{array}\right)
$$

where the $k$-column vector in the right-hand side is the $k^{\text {th }}$ column vector of the inverse matrix of $\left(a_{i j}\right)_{i, j=1, \ldots k}$. Hence, $\mathbf{v}_{k}$ does not depend on $\lambda_{i j}$ for $(i, j)$ satisfying $1 \leq i \leq j \leq n$, so that $\mathbf{v}_{k}=\mathbf{v}_{k}^{\prime}$. Thus 2 . is proved.

We define generalized flag spaces $\mathcal{F}_{n}^{\mathfrak{M}}$. Let

$$
\mathfrak{M}:=\left(m_{1}, \cdots, m_{\mu}\right)
$$


be a fixed, finite sequence of positive integers with $1 \leq m_{j} \leq n$ and $m_{1}+$ $\cdots+m_{\mu}=n$. Set $n_{j}:=m_{1}+m_{2}+\ldots+m_{j}$ for $j=1, \ldots, \mu$, and consider a nested sequence $\zeta$ of vector spaces in $\mathbb{C}^{n}$

$$
\zeta: \quad\{0\} \subset S_{n_{1}} \subset S_{n_{2}} \subset \cdots \subset S_{n_{\mu-1}} \subset \mathbb{C}^{n}
$$

where $S_{n_{j}}, j=1, \ldots, \mu-1$, is an $n_{j}$-dimensional vector space. Let $\mathcal{F}_{n}^{\mathfrak{M}}$ denote the set of all such sequences $\zeta$. We call $\mathcal{F}_{n}^{\mathfrak{m}}$ the $\mathfrak{M}$-flag space in $\mathbb{C}^{n}$. In particular, $\mathcal{F}_{n}^{\mathfrak{M}}$ coincides with $\mathcal{F}_{n}$ if $\mu=n$. Clearly $G L(n, \mathbb{C})$ acts transitively on $\mathcal{F}_{n}^{\mathfrak{M}}$. We fix the following point in $\mathcal{F}_{n}^{\mathfrak{M}}$ as the base point:

$$
O^{\mathfrak{M}}: \quad S_{n_{j}}=\left\{z_{n_{j}+1}=\ldots=z_{n}=0\right\}, \quad j=1, \ldots, \mu-1 .
$$

Thus the isotropy subgroup $H_{0}^{\mathfrak{M}}$ of $G L(n, \mathbb{C})$ for $O^{\mathfrak{M}}$ is the set of all matrices

$$
\left(\begin{array}{c|c|c}
h_{1} & (*) & (*) \\
\hline 0 & h_{j} & (*) \\
\hline 0 & 0 & h_{\mu}
\end{array}\right)
$$

where $h_{j} \in G L\left(m_{j}, \mathbb{C}\right), j=1, \ldots, \mu$. Hence

(i) $\mathcal{F}_{n}^{\mathfrak{M}} \approx G L(n, \mathbb{C}) / H_{0}^{\mathfrak{M}}$;

(ii) $H_{0} \subset H_{0}^{\mathfrak{M}}$ and $H_{0}^{\mathfrak{M}} / H_{0} \approx \mathcal{F}_{m_{1}} \times \cdots \times \mathcal{F}_{m_{\mu}}$, where $\mathcal{F}_{m_{j}}$ is the usual flag space in $\mathbb{C}^{m_{j}}$;

(iii) there exists a holomorphic projection $\pi_{\mathfrak{M}}: g H_{0} \in \mathcal{F}_{n} \mapsto g H_{0}^{\mathfrak{M}} \in \mathcal{F}_{n}^{\mathfrak{M}}$, such that

$$
\left(\pi_{\mathfrak{M}}\right)^{-1}(\zeta) \approx \mathcal{F}_{m_{1}} \times \cdots \times \mathcal{F}_{m_{\mu}}, \quad \zeta \in \mathcal{F}_{n}^{\mathfrak{M}}
$$

Our main result is the following.

Theorem 7.1. Let $D \Subset \mathcal{F}_{n}$ be a pseudoconvex domain with smooth boundary which is not Stein. Then there exists a unique sequence $\mathfrak{M}=\left(m_{1}, \ldots, m_{\mu}\right)$ with $1<\mu<n$ and a Stein domain $D_{0} \Subset \mathcal{F}_{n}^{\mathfrak{M}}$ with smooth boundary such that $D=\left(\pi_{\mathfrak{M}}\right)^{-1}\left(D_{0}\right)$. 
To prove the theorem, we isolate the main ingredient, Lemma 7.2. To specialists in Lie theory, this is the statement that any parabolic Lie subgroup of $G L(n, \mathbb{C})$ containing the Borel Lie subgroup $H_{0}$ must be of the form $H_{0}^{(\mathfrak{M})} \cap$ $G L(n, \mathbb{C})$ where $\mathfrak{M}$ is defined in (17.6). We give an elementary proof accessible to non-specialists. We first introduce some useful notation. For $1 \leq m \leq n-1$ and $q=n-m \geq 1$,

$O_{n}$ or $O\left(\right.$ resp. $\left.O_{q}\right)=$ the base point of $\mathcal{F}_{n}\left(\right.$ resp. $\left.\mathcal{F}_{q}\right)$;

$$
\begin{aligned}
M_{q}(\mathbb{C}) & =\text { the set of all } q \times q \text { square matrices; } \\
I_{q} & =\text { the identity in } M_{q} ; \\
M_{m, q}(\mathbb{C}) & =\text { the set of all } m \times q \text { matrices; } \\
H_{0}^{(q)} & =\text { the set of all upper triangular matrices in } M_{q}(\mathbb{C}) ; \\
M^{(m, n)} & =\left\{\left(\begin{array}{l|l}
X & Y \\
\hline 0 & 0
\end{array}\right) \in M_{n}(\mathbb{C}): X \in M_{m}(\mathbb{C}), Y \in M_{m, q}(\mathbb{C})\right\},
\end{aligned}
$$

and, for an element $X_{q} \in M_{q}(\mathbb{C})$ and a subset $\mathfrak{X}^{(q)} \subset M_{q}(\mathbb{C})$,

$$
\begin{aligned}
\omega_{n}\left(X_{q}\right) & =\left(\begin{array}{c|c}
0 & 0 \\
\hline 0 & X_{q}
\end{array}\right) \in M_{n}(\mathbb{C}) ; \\
\omega_{n}\left(\mathfrak{X}^{(q)}\right) & =\left\{\omega_{n}\left(X_{q}\right) \in M_{n}(\mathbb{C}): X_{q} \in \mathfrak{X}^{(q)}\right\} .
\end{aligned}
$$

We note that from (6.40), the Lie subalgebra $\mathfrak{H}_{0}$ of $\mathfrak{X}$ which corresponds to the isotopy group $H_{0}$ of $G L(n, \mathbb{C})$ for the identity $I$ can be written as $\mathfrak{H}_{0}=\left\{v_{X} \in \mathfrak{X}: X \in H_{0}^{(n)}\right\}$. We identify $\mathfrak{H}_{0}$ with $H_{0}^{(n)}$ just as we identify $\mathfrak{X}$ with $M_{n}(\mathbb{C})$. Then $H_{0}=H_{0}^{(n)} \cap G L(n, \mathbb{C})$.

Lemma 7.2. Let $\mathfrak{X}_{0} \subset M_{n}(\mathbb{C})$ have the following two properties:

(a) $\mathfrak{X}_{0}$ is a Lie subalgebra of $M_{n}(\mathbb{C})$ with $H_{0}^{(n)} \subset \mathfrak{X}_{0}$;

(b) let $X \in \mathfrak{X}_{0}$ and $A \in H_{0}$. Then any $Y \in M_{n}(\mathbb{C})$ which satisfies

$$
\left.\left[\frac{d A \exp t X\left(O_{n}\right)}{d t}\right]\right|_{t=0}=\left.\left[\frac{d \exp t Y\left(O_{n}\right)}{d t}\right]\right|_{t=0}
$$

belongs to $\mathfrak{X}_{0}$.

Assume $H_{0}^{(n)} \subsetneq \mathfrak{X}_{0} \subsetneq M_{n}(\mathbb{C})$. Then there exist a unique integer $\mathfrak{m}, 1 \leq \mathfrak{m} \leq$ $n-1$, and a unique subset $\mathfrak{X}_{0}^{(q)} \subset M_{q}(\mathbb{C})$ where $q=n-\mathfrak{m} \geq 1$ such that

(1) $\mathfrak{X}_{0}^{(q)}$ satisfies propeties $(a)$ and (b) with $n$ replaced by $q$; 
(2) $\mathfrak{X}_{0}=M^{(\mathfrak{m}, n)}+\omega_{n}\left(\mathfrak{X}_{0}^{(q)}\right)$.

Remark 7.1. According to T. Morimoto (private communication), condition $(a)$ implies condition $(b)$. We include $(b)$ as a hypothesized property to maintain the elementary character of the proof of the lemma. Note that $(b)$ is very similar in content with 2. of Lemma 6.4.

Proof. The uniqueness is clear. To verify the existence of $\mathfrak{X}_{0}^{(q)}$ satisfying (1) and (2) we divide the proof into seven short steps. For $1 \leq k \leq \nu \leq n$ we write $X_{\nu 1} \in M_{n}(\mathbb{C})$ for the matrix whose $(\nu, 1)$-entry is 1 and whose other entries are all 0 .

$1^{\text {st }}$ step. Fix $\nu$ with $2 \leq \nu \leq n$. If $X_{\nu 1} \in \mathfrak{X}_{0}$, then $\mathfrak{X}_{0}$ contains all matrices of the form

$$
Y_{\left(a_{2}, \ldots, a_{\nu}\right)}=\left(\begin{array}{c|l}
0 & \\
a_{2} & \\
\vdots &
\end{array}\right), \quad a_{j} \in \mathbb{C}, j=2, \ldots, \nu
$$

where the missing entries are all 0.

Proof. Let $a_{j} \in \mathbb{C}, j=1, \ldots, \nu$ with $a_{\nu} \neq 0$. We consider

$$
A=\left(\begin{array}{cccc|c}
1 & & & a_{1} & \\
& \ddots & & \vdots & \\
& & 1 & a_{\nu-1} & \\
& & a_{\nu} & \\
\hline & & & & I_{n-\nu}
\end{array}\right) \in H_{0} .
$$

We let

$$
\mathbf{a}(\mathbf{t}):=\left(\mathbf{a}_{1}(t) ; \mathbf{a}_{2}(t) ; \ldots ; \mathbf{a}_{n-1}(t)\right)
$$

represent the point $A \exp t X_{\nu 1}(O)$ in $\mathcal{F}_{n}$ in terms of the standard local coordinates at $O$. Then we have by (7.4)

$$
\begin{aligned}
& \mathbf{a}_{1}(t)=\frac{1}{1+a_{1} t}\left(a_{2} t, \ldots, a_{\nu-1} t, a_{\nu} t, 0, \ldots, 0\right) \\
& \mathbf{a}_{j}(t)=(0, \ldots, 0), \quad j=2, \ldots, n-1
\end{aligned}
$$


It follows that the direction of the curve $A \exp t X_{\nu 1}(O)$ at $O$ in $\mathcal{F}_{n}$ is

$$
\mathbf{v}=\left(a_{2}, \ldots, a_{\nu}, 0, \ldots, 0 ; 0, \ldots, 0 ; \ldots ; 0\right) .
$$

From 1. in Lemma 7.1 the curve $\exp t Y_{\left(a_{2}, \ldots, a_{\nu}\right)}(O)$ at $O$ in $\mathcal{F}_{n}$ has the same direction $\mathbf{v}$. Since $X_{\nu 1} \in \mathfrak{X}_{0}$, it follows from property (b) in Lemma 7.2 that $Y_{\left(a_{2}, \ldots, a_{\nu}\right)} \in \mathfrak{X}_{0}$. This proves the $1^{\text {st }}$ step in the case where $a_{\nu} \neq 0$. Suppose now $a_{\nu}=0$. From the previous case, we have $Y_{\left(a_{2}, \ldots, a_{\nu-1}, \varepsilon\right)} \in \mathfrak{X}_{0}$ for any $\varepsilon \in \mathbb{C} \backslash\{0\}$. Since $\mathfrak{X}_{0}$ is a vector subspace of $M_{n}(\mathbb{C})$, letting $\varepsilon \rightarrow 0$ we see that the $1^{\text {st }}$ step is true for $a_{\nu}=0$.

$2^{\text {nd }}$ step. Fix $\nu$ with $2 \leq \nu \leq n$. If $X_{\nu 1} \in \mathfrak{X}_{0}$, then $\mathfrak{X}_{0}$ contains all matrices of the form

$$
Z_{\left(a_{1}, \ldots, a_{\nu-1}\right)}=\left(\begin{array}{ccccccc}
0 & & & & & & \\
& 0 & & & & & \\
& & \ddots & & & & \\
& & & 0 & & & \\
a_{1} & a_{2} & \cdots & a_{\nu-1} & 0 & \cdots & 0 \\
& & & & & \ddots & \\
& & & & & & 0
\end{array}\right) .
$$

Proof. It suffices to prove $Z_{\left(1, a_{2}, \ldots, a_{\nu-1}\right)} \in \mathfrak{X}_{0}$ for any $a_{2}, \ldots, a_{\nu} \in \mathbb{C}$. We consider

$$
A=\left(\begin{array}{cccc|c}
1 & -a_{2} & \ldots & -a_{\nu} & \\
& 1 & & & \\
& & \ddots & & \\
& & 1 & \\
\hline & & & & I_{n-\nu}
\end{array}\right) \in H_{0} .
$$

We let

$$
\mathbf{a}(\mathbf{t}):=\left(\mathbf{a}_{1}(t) ; \mathbf{a}_{2}(t) ; \ldots ; \mathbf{a}_{n-1}(t)\right)
$$

denote the point $A \exp t X_{\nu 1}(O)$ in $\mathcal{F}_{n}$ in terms of the standard local coordinates at $O$. Then we have by (7.4)

$$
\mathbf{a}_{j}(t)=\frac{1}{1-a_{\nu} t}\left(0, \ldots, 0, a_{j} t, 0, \ldots, 0\right), \quad j=1,2, \ldots, \nu-1,
$$

where we set $a_{1}=1$ and $a_{j} t$ is located in the $(\nu-j)$-th slot. For $\nu \leq j \leq n$ we have $\mathbf{a}_{j}(t)=(0, \ldots, 0)$. It follows that the direction $\mathbf{v}$ of the analytic curve $A \exp t X_{\nu 1}(O)$ at $O$ in $\mathcal{F}_{n}$ is

$$
\mathbf{v}=\left(0, \ldots, 0, a_{1}, 0, \ldots, 0 ; \cdots ; 0, \ldots, 0, a_{\nu-1}, 0, \ldots, 0 ; 0, \ldots, 0 ; \cdots ; 0\right) .
$$


From 1. in Lemma 7.1 and (b) in Lemma 7.2 we have $Z_{\left(1, a_{2}, \ldots, a_{\nu-1}\right)} \in \mathfrak{X}_{0}$.

Let $1 \leq k<\nu \leq n$ and write $X_{\nu k} \in M_{n}(\mathbb{C})$ for the matrix whose $(\nu, k)$ entry is 1 and whose other entries are all 0 . By the $1^{\text {st }}$ and $2^{\text {nd }}$ steps we have:

$$
X_{\nu 1} \in \mathfrak{X}_{0} \text { implies } X_{i j} \in \mathfrak{X}_{0}, \quad 1 \leq j<i \leq \nu
$$

$3^{\text {rd }}$ step. Fix $\nu$ with $2 \leq \nu \leq n$. Assume that $\mathfrak{X}_{0}$ contains a matrix of the form

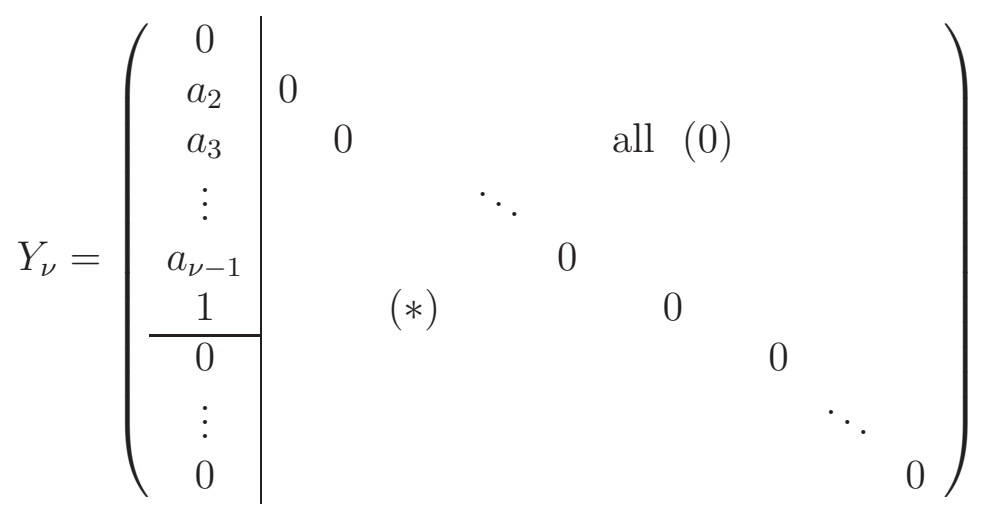

where the $\frac{(n-2)(n-1)}{2}$ entries $(*)$ are complex numbers. Then $\mathfrak{X}_{0}$ contains a matrix of the form

$$
Y_{\nu}^{*}=\left(\begin{array}{c|cccccccc}
0 & & & & & & & & \\
0 & 0 & & & & & & & \\
0 & & 0 & & & & \text { all }(0) & & \\
\vdots & & & \ddots & & & & & \\
0 & & & & 0 & & & & \\
1 & & (\star) & & & 0 & & & \\
\hline 0 & & & & & & 0 & & \\
\vdots & & & & & & & \ddots & \\
0 & & & & & & & & 0
\end{array}\right)
$$

where the $(\nu, 1)$-entry is 1 and the entries $(\star)$ are complex numbers (i.e., $Y_{\nu}^{*}$ is a matrix of the same form as $Y_{\nu}$ but with the entries $\left.a_{2}=\cdots=a_{\nu-1}=0\right)$. 
Proof. We consider

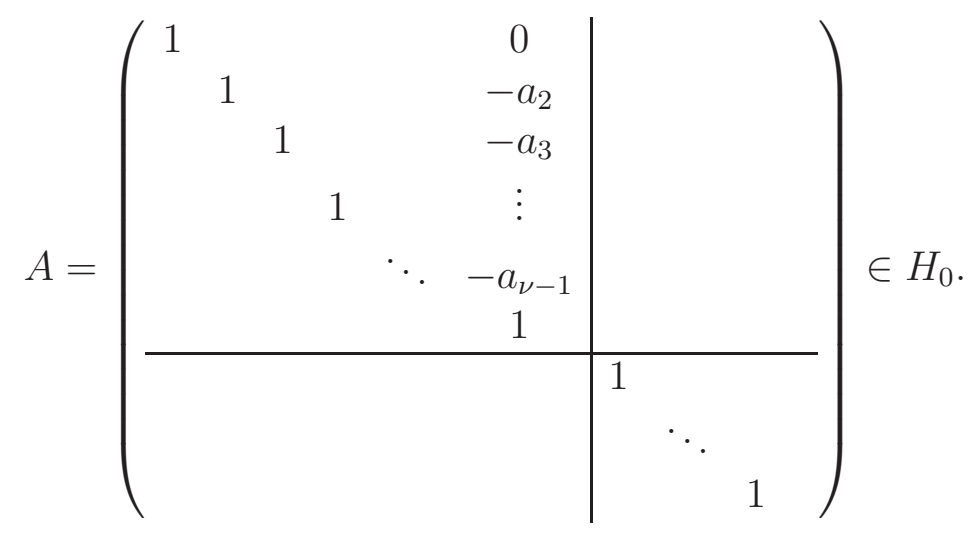

Then the direction $\mathbf{v}$ of the curve $A \exp t Y_{\nu}(O)$ at $O$ is of the form

$$
\mathbf{v}=\left(0, \ldots, 0,1,0 \ldots, 0 ; b_{32}, \ldots b_{n 2} ; \ldots ; b_{n n-1}\right)
$$

where $b_{i j}, 2 \leq j<i \leq n$ are complex numbers. From 1. in Lemma 7.1 and (b) in Lemma 7.2, $Y_{\nu}^{*}$ in (7.10) for $(\star)=\left(b_{i j}\right)$ belongs to $\mathfrak{X}_{0}$.

$4^{\text {th }}$ step. Fix $\nu$ with $2 \leq \nu \leq n$. If $\mathfrak{X}_{0}$ contains at least one matrix $Y_{\nu}^{*} \in M_{n}(\mathbb{C})$ of the form (7.10), then $X_{\nu 1} \in \mathfrak{X}_{0}$.

Proof. We set $(\star)=\left(a_{i j}\right), 2 \leq j<i \leq n-1$ in $Y_{\nu}^{*}$ in (7.10). For a given sequence

$$
\{\mathbf{M} ; \varepsilon\}: \quad M_{2} \gg \ldots \gg M_{\nu-1} \gg 1 \gg \varepsilon_{1} \gg \ldots \gg \varepsilon_{n-\nu}>0,
$$

we consider the diagonal matrix

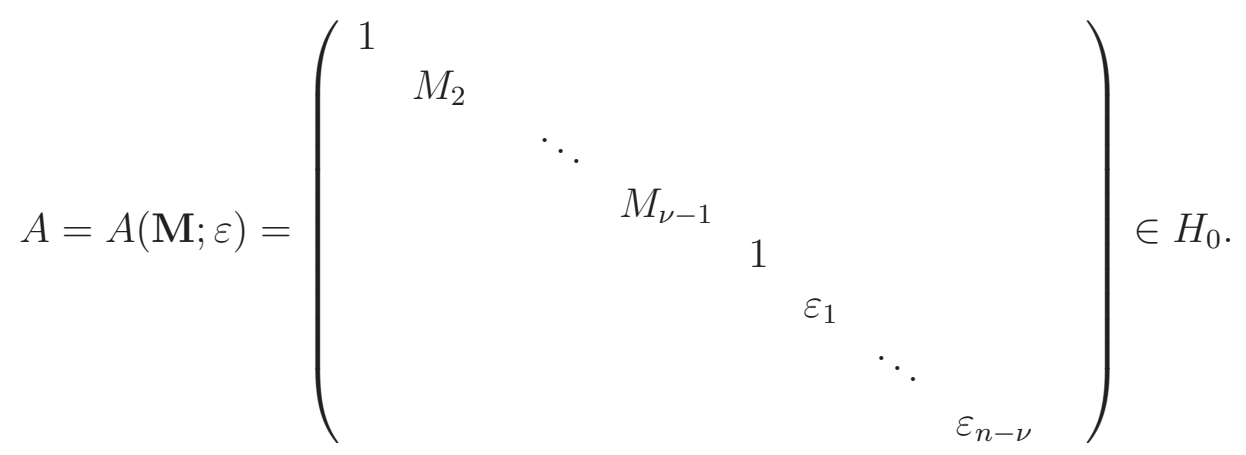


By direct calculation, $A \exp t Y_{\nu}^{*}$ is equal to

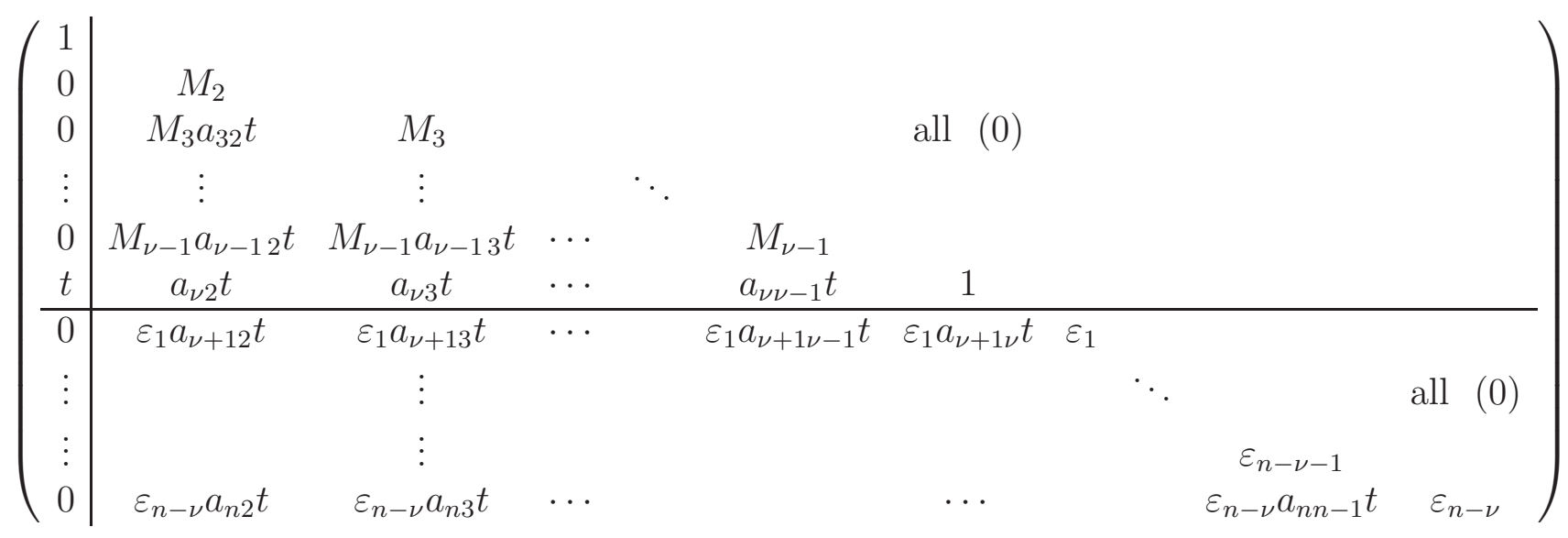

up to terms of order $O\left(t^{2}\right)$. We write

$$
\mathbf{v}=\left(\mathbf{v}_{1} ; \mathbf{v}_{2} ; \ldots: \mathbf{v}_{\nu} ; \ldots,: \mathbf{v}_{n-1}\right)
$$

for the direction of $A \exp t Y_{\nu}^{*}(O)$ at $O$. Using (7.4) we have $\mathbf{v}_{1}=(0, \ldots, 0,1,0, \ldots, 0)$, where 1 is in the $(\nu-1)$-st slot. We also have

$$
\mathbf{v}_{2}=\frac{1}{M_{2}}\left(M_{3} a_{32}, \ldots, M_{\nu-1} a_{\nu-12}, a_{\nu 2}, \varepsilon_{1} a_{\nu+1,2}, \ldots, \varepsilon_{n-\nu} a_{n 2}\right) .
$$

Thus by taking

$$
M_{2} \gg M_{3}, \ldots, M_{\nu-1}, \varepsilon_{1}, \ldots, \varepsilon_{n-\nu}>0
$$

we can make $\mathbf{v}_{2}$ as close to the $(n-2)$-row vector $\mathbf{0}$ as we like.

Similar results hold for $\mathbf{v}_{j}, j=3, \ldots, \nu-1$; i.e., by taking

$$
M_{j} \gg M_{j+1}, \ldots, M_{\nu-1}, \varepsilon_{1}, \ldots, \varepsilon_{n-\nu}>0
$$

we can make $\mathbf{v}_{j}$ as close to the $(n-j)$-row vector $\mathbf{0}$ as we like. We have

$$
\mathbf{v}_{\nu}=\left(\varepsilon_{1} a_{\nu+1 \nu}, \ldots, \varepsilon_{n-\nu} a_{n \nu}\right)
$$

thus by taking

$$
1 \gg \varepsilon_{1}, \ldots, \varepsilon_{n-\nu}>0
$$

we can make $\mathbf{v}_{\nu}$ as close to the $(n-\nu)$-row vector $\mathbf{0}$ as we like. We have

$$
\mathbf{v}_{\nu+1}=\frac{1}{\varepsilon_{1}}\left(\varepsilon_{2} a_{\nu+2 \nu+1}, \ldots, \varepsilon_{n-\nu} a_{n \nu+1}\right)
$$


Thus by taking

$$
\varepsilon_{1} \gg \varepsilon_{2}, \ldots, \varepsilon_{n-\nu}>0
$$

we can make $\mathbf{v}_{\nu+1}$ as close to the $(n-\nu-1)$-row vector $\mathbf{0}$ as we like.

Similar results hold for $\mathbf{v}_{j}, j=\nu+2, \ldots, n-1$. For example, for $\mathbf{v}_{n-1}$, we have $\mathbf{v}_{n-1}=\frac{1}{\varepsilon_{n-\nu-1}} \varepsilon_{n-\nu} a_{n n-1}$, so that, by taking $\varepsilon_{n-\nu-1} \gg \varepsilon_{n-\nu}>0$ we make $\mathbf{v}_{n-1}$ as close to the complex number 0 as we like.

Therefore by taking

$$
\{\mathbf{M} ; \varepsilon\}: \quad M_{2} \gg \ldots \gg M_{\nu-1} \gg 1 \gg \varepsilon_{1} \gg \ldots \gg \varepsilon_{n-\nu}>0
$$

and considering $A=A(\mathbf{M} ; \varepsilon) \in H_{0}$ as in (17.11), the direction $\mathbf{v}$ of $A \exp t Y_{\nu}^{*}(O)$ at $O$ in $\mathcal{F}_{n}$ can be made as close to

$$
\mathbf{U}:=(0, \ldots, 0,1,0, \ldots 0 ; 0, \ldots, 0 ; \cdots ; 0) .
$$

as we like. Since $\mathbf{U}$ is the direction of $\exp t X_{\nu 1}(0)$ at $t=0$, it follows from 1. in Lemma 7.1 and (b) in Lemma 7.2 that $X_{\nu 1} \in \mathfrak{X}_{0}$.

As the integer $\mathfrak{m}$ in Lemma 7.2 we take

$$
\mathfrak{m}:=\max _{X \in \mathfrak{X}_{0}}[\max \{i: \operatorname{each}(j, 1) \text {-entry of } X \text { with } j>i \text { is } 0\}] .
$$

Since $H_{0}^{(n)} \subsetneq \mathfrak{X}_{0}$ we have $\mathfrak{m} \geq 2$; on the other hand, from the $4^{\text {th }}$ step, we have $\mathfrak{m} \leq n-1$.

$5^{\text {th }}$ step. The number $\mathfrak{m}$ has the following properties:

(i) $\left\{\left(\begin{array}{c|c}X_{\mathfrak{m}} & 0 \\ \hline 0 & 0\end{array}\right) \in M_{n}(\mathbb{C}): X_{\mathfrak{m}} \in M_{\mathfrak{m}}(\mathbb{C})\right\}+H_{0}^{(n)} \subset \mathfrak{X}_{0} ;$

(ii) for any $X \in \mathfrak{X}_{0}$, each $(i, j)$-entry with $\mathfrak{m}+1 \leq i \leq n ; 1 \leq j \leq \mathfrak{m}$ is 0 .

Proof. By the $3^{\text {rd }}$ and the $4^{\text {th }}$ steps we have $X_{\mathfrak{m} 1} \in \mathfrak{X}_{0}$; then (17.8) implies assertion (i). We shall prove (ii) by contradiction. Assume that there exists $A=\left(a_{i j}\right) \in \mathfrak{X}_{0}$ with $a_{i_{0} j_{0}} \neq 0$ for some $\mathfrak{m}+1 \leq i_{0} \leq n ; 1 \leq j_{0} \leq \mathfrak{m}$. Since $1 \leq j_{0} \leq \mathfrak{m}$, it follows from (i) that $X_{j_{0}} \in \mathfrak{X}_{0}$. Now $\mathfrak{X}_{0}$ is a Lie subalgebra of $M_{n}(\mathbb{C})$; thus $\mathfrak{X}_{0}$ contains the Lie bracket $\left[A, X_{j_{0}}\right]$. A calculation shows that the $\left(i_{0}, 1\right)$-entry of $\left[A, X_{j_{0}}\right]$ is equal to $a_{i_{0} j_{0}} \neq 0$. Since $i_{0}>\mathfrak{m}$, this contradicts the definition of $\mathfrak{m}$.

Note that we only used the fact that $\mathfrak{X}_{0}$ is closed under Lie brackets and the structure of $H_{0}$ in the proof of Lemma 7.2. We set $q=n-\mathfrak{m} \geq 1$ and we consider $\mathbb{C}^{n}=\mathbb{C}^{\mathfrak{m}} \times \mathbb{C}^{q}$. 
$6^{\text {th }}$ step. We define

$$
\mathfrak{X}_{0}^{(q)}:=\left\{Y_{q} \in M_{q}(\mathbb{C}): \omega_{n}\left(Y_{q}\right) \in \mathfrak{X}_{0}\right\} .
$$

Then $\mathfrak{X}_{0}^{(q)}$ satisfies (1) in Lemma $\left[7.2\right.$, i.e., $\mathfrak{X}_{0}^{(q)}$ satisfies propeties $(a)$ and $(b)$ with $n$ replaced by $q$.

Proof. Property (a) with $n$ replaced by $q$ clearly holds. To prove (b), let $X_{q} \in \mathfrak{X}_{0}^{(q)}, A_{q} \in H_{0}^{(q)} \cap G L(q, \mathbb{C})$ and let $Y_{q} \in M_{q}(\mathbb{C})$ satisfy

$$
\left.\left[\frac{d A_{q} \exp t X_{q}\left(O_{q}\right)}{d t}\right]\right|_{t=0}=\left.\left[\frac{d \exp t Y_{q}\left(O_{q}\right)}{d t}\right]\right|_{t=0} .
$$

To prove our claim that $Y_{q} \in \mathfrak{X}_{0}^{(q)}$, we first write

$$
\mathbf{V}:=\left(\mathbf{v}_{1} ; \mathbf{v}_{2} ; \cdots ; \mathbf{v}_{q-1}\right) \text { and } \mathbf{v}_{k}=\left(v_{k+1 k}, \ldots, v_{q k}\right), 1 \leq k \leq q-1,
$$

for the direction of the curve $A_{q} \exp t X_{q}\left(O_{q}\right)$ at $O_{q}$ in $\mathcal{F}_{q}$ in terms of the standard local coordinates $\mathbf{t} \in \mathbb{C}^{Q}$. Here $Q=\frac{q(q-1)}{2}$. If we set

$$
Y_{q}^{\prime}:=\left(\begin{array}{lllll}
0 & & & & \\
v_{21} & 0 & & & \\
v_{31} & v_{32} & 0 & & \\
\vdots & \vdots & \ddots & \ddots & \\
v_{q 1} & v_{q 2} & \cdots & v_{q q-1} & 0
\end{array}\right) \in M_{q}(\mathbb{C})
$$

then 1. in Lemma 7.1 with $n$ replaced by $q$ together with (7.13) implies $Y_{q}=Y_{q}^{\prime}+B_{q}^{*}$ for some $B_{q}^{*} \in H_{0}^{(q)}$.

We next consider

$$
X:=\omega_{n}\left(X_{q}\right) \in M_{n}(\mathbb{C}), \quad A:=\left(\begin{array}{c|c}
I_{\mathfrak{m}} & 0 \\
\hline 0 & A_{q}
\end{array}\right) \in H_{0},
$$

so that $X \in \mathfrak{X}_{0}$. By (7.5), the direction of the curve $A \exp t X(O)$ at $O$ in $\mathcal{F}_{n}$ is

$$
\mathbf{U}=\left(\mathbf{0}_{n-1} ; \ldots ; \mathbf{0}_{n-\mathfrak{m}} ; \mathbf{v}_{1} ; \mathbf{v}_{2} ; \ldots ; \mathbf{v}_{q-1}\right),
$$

where $\mathbf{0}_{k}$ is the zero $k$-row vector. It follows from 1 . in Lemma 7.1 and (b) in Lemma 7.2 that $\omega_{n}\left(Y_{q}^{\prime}\right) \in \mathfrak{X}_{0}$. Consequently, $\omega_{n}\left(Y_{q}\right)=\omega_{n}\left(Y_{q}^{\prime}+B_{q}^{*}\right)=$ $Y^{\prime}+\omega_{n}\left(B_{q}^{*}\right) \in \mathfrak{X}_{0}$, which is what we needed to show.

$7^{\text {th }}$ step. Property (2) in Lemma 7.2 holds for $\mathfrak{X}_{0}^{(q)}$ defined in (7.12).

Proof. Let $X \in \mathfrak{X}_{0}$. By (ii) in the $5^{\text {th }}$ step, $X$ is of the form

$$
X=\left(\begin{array}{c|c}
X_{\mathfrak{m}} & X_{\mathfrak{m} q} \\
\hline 0 & X_{q}
\end{array}\right), \quad X_{\mathfrak{m}} \in M_{\mathfrak{m}}(\mathbb{C}), X_{\mathfrak{m} q} \in M_{\mathfrak{m}, q}(\mathbb{C}), X_{q} \in M_{q}(\mathbb{C}) .
$$


Since $\mathfrak{X}_{0}$ is a vector subspace of $M_{n}(\mathbb{C})$, it follows from (i) in the $5^{\text {th }}$ step that $\omega_{n}\left(X_{q}\right) \in \mathfrak{X}_{0}$; thus $X_{q} \in \mathfrak{X}_{0}^{(q)}$ and $X \in M^{(\mathfrak{m}, n)}+\omega_{n}\left(X_{q}\right)$, so that $\mathfrak{X}_{0} \subset$ $M^{(\mathfrak{m}, n)}+\omega_{n}\left(\mathfrak{X}_{0}^{(q)}\right)$. The reverse inclusion follows from (i) of the $5^{\text {th }}$ step and the definition of $\mathfrak{X}_{0}^{(q)}$. The $7^{\text {th }}$ step, and Lemma 7.2, are proved.

Proof of Theorem 7.1. It suffices to prove the theorem under the assumption that $D$ contains the base point $O$ in $\mathcal{F}_{n}$. We consider the $c$ Robin function $\Lambda(z)$ for $D$ and define

$$
\mathfrak{X}_{0}:=\left\{X \in M_{n}(\mathbb{C}):\left.\left[\frac{\partial^{2} \Lambda(\exp t X(O))}{\partial t \partial \bar{t}}\right]\right|_{t=0}=0\right\} .
$$

We see from the assumption for $D$ and Remark 6.1 that $H_{0}^{(n)} \subsetneq \mathfrak{X}_{0} \subsetneq M_{n}(\mathbb{C})$. Using 1. and 2. in Lemma 6.4 for general homogeneous spaces we see that $\mathfrak{X}_{0}$ satisfies properties (a) and (b) in Lemma 7.2. Using Lemma 7.2 inductively, we can find $\mathfrak{M}=\left(m_{1}, \cdots, m_{\mu}\right)$ with $1<\mu<n$ such that $\mathfrak{X}_{0}$ is the subset $H_{0}^{(\mathfrak{M})}$ of $M_{n}(\mathbb{C})$ which consists of all matrices of the form

$$
\left(\begin{array}{c|c|c}
h_{m_{1}} & (*) & (*) \\
\hline 0 & h_{m_{j}} & (*) \\
\hline 0 & 0 & h_{m_{\mu}}
\end{array}\right)
$$

where $h_{m_{j}} \in M_{m_{j}}(\mathbb{C}), j=1, \ldots, \mu$, and each $(*)$ is an arbitrary element in the corresponding space $M_{m_{j}, m_{k}}(\mathbb{C})$ (here $m_{j}<m_{k}$ ). It follows that the integral manifold $\Sigma_{0}$ of the Lie subalgebra $\mathfrak{X}_{0}$ passing through $O$ in $G L(n, \mathbb{C})$, i.e., the connected Lie subgroup of $G L(n, \mathbb{C})$ corresponding to $\mathfrak{X}_{0}$, is $H_{0}^{(\mathfrak{M})} \cap G L(n, \mathbb{C})$ and hence it is equal to the isotropy subgroup $H_{0}^{\mathfrak{M}}$ of $G L(n, \mathbb{C})$ at the identity $I$ for the generalized flag space $\mathcal{F}_{n}^{\mathfrak{M}}$. Thus, for the flag space $\mathcal{F}_{n}$, the space $M_{0}:=G L(n, \mathbb{C}) / \Sigma_{0}$, which was considered in the proof of Theorem 6.3 for general homogeneous spaces, coincides with the space $\mathcal{F}_{n}^{\mathfrak{M}}$. Consequently, the projection $\pi_{0}$ and the analytic set $\sigma \Subset D \subset M$ defined in $1-b$. in Theorem 6.3 coincide with the projection $\pi_{\mathfrak{M}}: A H_{0} \in$ $\mathcal{F}_{n} \rightarrow A \Sigma_{0}=A H_{0}^{\mathfrak{M}} \in \mathcal{F}_{n}^{\mathfrak{M}}$ (where $A \in G L(n, \mathbb{C})$ ) and the analytic set $H_{0}^{\mathfrak{M}} / H_{0} \Subset D \subset \mathcal{F}_{n}$. Therefore, $\pi_{\mathfrak{M}}^{-1}(\zeta) \approx \pi_{\mathfrak{M}}{ }^{-1}\left(O^{\mathfrak{M}}\right)=H_{0}^{\mathfrak{M}} / H_{0} \approx \prod_{j=1}^{\mu} \mathcal{F}_{m_{j}}$ for $\zeta \in \mathcal{F}_{n}^{\mathfrak{M}}$. Using $1-b$. in Theorem 6.3, there exists a Stein domain 
$D_{0} \Subset \mathcal{F}_{n}^{\mathfrak{M}}$ with smooth boundary such that $D=\pi_{\mathfrak{M}}^{-1}\left(D_{0}\right)$. Theorem 7.1 is completely proved.

The following remark is from T. Ueda. Consider two generalized flag spaces $\mathcal{F}_{n}^{\mathfrak{M}}$ and $\mathcal{F}_{n}^{\mathfrak{L}}$ in $\mathbb{C}^{n}$, where $\mathfrak{M}=\left(m_{1}, \ldots, m_{\mu}\right), \mathfrak{L}=\left(l_{1}, \ldots, l_{\nu}\right), \mu>\nu$, and

$$
l_{1}=m_{1}+m_{2}+\ldots+m_{j_{1}}, \ldots, l_{\nu}=m_{j_{\nu-1}+1}+\ldots+m_{\mu} .
$$

We introduce the notation $\mathfrak{M} \prec \mathfrak{L}$ for this situation. Then we have the canonical projection

$$
\pi_{\mathfrak{L}}^{\mathfrak{M}}: g H_{0}^{\mathfrak{M}} \in \mathcal{F}_{n}^{\mathfrak{M}} \mapsto g H_{0}^{\mathfrak{L}} \in \mathcal{F}_{n}^{\mathfrak{L}},
$$

where $H_{0}^{\mathfrak{M}}$ is the isotropy subgroup of $G$ for $\mathcal{F}_{n}^{\mathfrak{M}}$ at the base point $O^{\mathfrak{M}}$. Thus, for each $z \in \mathcal{F}_{n}^{\mathfrak{L}}$,

$$
\left(\pi_{\mathfrak{L}}^{\mathfrak{M}}\right)^{-1}(z) \approx \mathcal{F}_{l_{1}}^{\mathfrak{M}_{1}} \times \ldots \times \mathcal{F}_{l_{\nu}}^{\mathfrak{M}_{\nu}} \quad \text { as complex manifolds }
$$

where $\mathfrak{M}_{k}=\left(m_{j_{k}+1}, \ldots, m_{j_{k}}\right), k=1,2, \ldots, \nu$. If $\mathfrak{M}=(1, \ldots, 1)$, i.e., $\mathcal{F}_{n}^{\mathfrak{M}}=$ $\mathcal{F}_{n}$, we simply write $\pi_{\mathfrak{L}}^{\mathfrak{M}}=\pi_{\mathfrak{L}}$.

We have the following result.

Corollary 7.1. Let $D$ be a pseudoconvex domain with smooth boundary in $\mathcal{F}_{n}^{\mathfrak{M}}$ which is not Stein. Then there exists a unique $\mathfrak{L}$ such that $\mathfrak{M} \prec \mathfrak{L}$ and a Stein domain $D_{0}$ in $\mathcal{F}_{n}^{\mathfrak{L}}$ with smooth boundary such that $D=\left(\pi_{\mathfrak{L}}^{\mathfrak{M}}\right)^{-1}\left(D_{0}\right)=$ D.

Proof. We assume that the base point $O^{\mathfrak{M}}$ of $\mathcal{F}_{n}^{\mathfrak{M}}$ is contained in $D$. Define $\widetilde{D}:=\left(\pi_{\mathfrak{M}}\right)^{-1}(D) \subset \mathcal{F}_{n}$. Then $\widetilde{D}$ is a pseudoconvex domain with smooth boundary in $\mathcal{F}_{n}$. We consider the $c$-Robin function $\lambda(z)$ for $\widetilde{D}$ and define

$$
\mathfrak{X}_{0}=\left\{X \in \mathfrak{X}:\left.\left[\frac{\partial^{2} \lambda(\exp t X(O))}{\partial t \partial \bar{t}}\right]\right|_{t=0}\right\}
$$

where $O$ is the base point of $\mathcal{F}_{n}$ and $\mathfrak{X}$ is the Lie algebra consisting of all left-invariant holomorphic vector fields on $\mathcal{F}_{n}$. Let $\Sigma$ be the Lie subgroup of $G L(n, \mathbb{C})$ which corresponds to $\mathfrak{X}_{0}$. Finally, let $\mathfrak{g}_{0}^{\mathfrak{M}}$ be the Lie subalgebra corresponding to $H_{0}^{\mathfrak{M}} \subset G L(n, \mathbb{C})$. Since $\lambda=$ const. on the fiber $\left(\pi_{\mathfrak{M}}\right)^{-1}\left(O^{\mathfrak{M}}\right)$, we have $\mathfrak{g}_{0}^{\mathfrak{M}} \subset \mathfrak{X}_{0}$ (we identify both sets as subsets of $M_{n}(\mathbb{C})$ ). Hence $H_{0}^{\mathfrak{M}} \subset \Sigma(O)$. Fix $\zeta \in D$ and take a point $z \in\left(\pi_{\mathfrak{M}}\right)^{-1}(\zeta)$. Then

$$
\widehat{\lambda}(\zeta):=\lambda(z)
$$

is well-defined and $-\widehat{\lambda}$ is a plurisubharmonic exhaustion function on $D$. Since $D$ is not Stein, there exists $\zeta_{0} \in D$ and a direction $\mathbf{a} \in \mathbb{C}^{N} \backslash\{0\}$ (here 
$\left.N=\operatorname{dim} \mathcal{F}_{n}^{\mathfrak{M}}\right)$ such that $\left.\left[\frac{\partial^{2} \widehat{\lambda}(\zeta+\mathbf{a} t)}{\partial t \partial \bar{t}}\right]\right|_{t=0}=0$. Thus if we take $z_{0} \in\left(\pi_{\mathfrak{M}}\right)^{-1}\left(\zeta_{0}\right)$, then $\left.\left[\frac{\partial \lambda\left(\zeta_{0}+\mathbf{a}^{\prime} t\right)}{\partial t \partial \bar{t}}\right]\right|_{t=0}=0$ where $\pi_{\mathfrak{M}}\left(\zeta_{0}+\mathbf{a}^{\prime} t\right)=z_{0}+\mathbf{a} t$. If we take $X \in \mathfrak{X}$ such that $\left.\left[\frac{d \exp t X(O)}{d t}\right]\right|_{t=0}=\mathbf{a}^{\prime}$, then $X \in \mathfrak{X}_{0} \backslash \mathfrak{g}_{0}^{\mathfrak{M}}$. It follows from the proof of Theorem 7.1 that there exists $\mathfrak{L}$ with $\mathfrak{M} \prec \mathfrak{L}$ and a Stein domain $D_{0}$ with smooth boundary in $\mathcal{F}_{n}^{\mathfrak{L}}$ such that $\widetilde{D}=\left(\pi_{\mathfrak{L}}\right)^{-1}\left(D_{0}\right)$. Since $\mathfrak{M} \prec \mathfrak{L}$, it follows from the definition of $\widetilde{D}$ that $\pi_{\mathfrak{L}}^{\mathfrak{M}}=\left.\left.\pi_{\mathfrak{L}}\right|_{\widetilde{D}} \circ\left(\pi_{\mathfrak{M}}\right)^{-1}\right|_{D}$ is well-defined; $\pi_{\mathfrak{L}}^{\mathfrak{M}}: D \mapsto D_{0}$ is surjective; and $D=\left(\tilde{\pi}_{\mathfrak{L}}^{\mathfrak{M}}\right)^{-1}\left(D_{0}\right)$, as claimed.

T. Ueda has another proof of this corollary following ideas in the paper [1] (which is based on [19]).

\section{Appendix A}

We discuss the three point property and the spanning property for projective space, Grassmannian manifolds, and flag spaces. Our first observation is elementary:

1. Projective space $M=\mathbb{P}^{n}$ with Lie transformation group $G=G L(n, \mathbb{C})$ satisfies the three point property.

On the other hand, we have:

2. The Grassmannian manifold $M=G(k, n)$ with Lie transformation group $G=G L(n, \mathbb{C})$ and $n \geq 4, n-2 \geq k \geq 2$ does not satisfy the three point property.

Recall that $M$ is the set of all $k$-dimensional subspaces of $\mathbb{C}^{n}$; we use coordinates $x=\left(x_{1}, \ldots, x_{n}\right)$. Given $z \in M$, we may write $z$ as

$$
z:\left(\begin{array}{c}
x_{1} \\
\vdots \\
x_{n}
\end{array}\right)=\left(\begin{array}{ccc}
\alpha_{11} & \cdots & \alpha_{1 k} \\
& \ddots & \\
\alpha_{n 1} & \cdots & \alpha_{n k}
\end{array}\right)\left(\begin{array}{c}
\zeta_{1} \\
\vdots \\
\zeta_{k}
\end{array}\right), \quad \text { i.e., } \quad{ }^{t} x=\alpha{ }^{t} \zeta,
$$

where $\zeta=\left(\zeta_{1}, \ldots, \zeta_{k}\right) \in \mathbb{C}^{k}$. Then $g=\left(g_{i j}\right) \in G$ acts on $z$ as follows:

$$
g(z):{ }^{t} x=(g \cdot \alpha){ }^{t} \zeta \in M .
$$

We let $O$ denote the point in $M$ defined as $x_{k+1}=\ldots=x_{n}=0$. Then the isotopy subgroup $H_{0}$ of $G$ for the point $O$ consists of all elements in $G$ of the form $\left(\begin{array}{c|c}A_{k} & * \\ \hline O & B_{n-k}\end{array}\right)$, where $A_{k}$ and $B_{n-k}$ are nonsingular square matrices of order $k$ and $n-k$.

We prove that $(G, M)$ does not satisfy the three point property by contradiction. Thus we take the following three points:

$$
z_{0}=O ; z_{1}: x_{k}=x_{k+2}=\ldots=0 ; z_{2}: x_{k-1}=x_{k}=\ldots=0 .
$$


Then we have

$$
z_{0} \cap z_{1}: x_{k}=x_{k+1}=\ldots=0 \text { and } z_{0} \cap z_{2}: x_{k-1}=x_{k}=\ldots=0 .
$$

Assuming the three point property holds, we can find $g \in H_{0}^{\prime}$ so that $g(O)=$ $O$ and $g\left(z_{1}\right)=z_{2}$. Since $g$ is one-to-one, we have $g\left(z_{0} \cap z_{1}\right)=g\left(z_{0}\right) \cap g\left(z_{1}\right)=$ $z_{0} \cap z_{2}$, which is $(k-2)$-dimensional. On the other hand, $g\left(z_{0} \cap z_{1}\right)$ is $(k-1)$ dimensional, since $z_{0} \cap z_{1}$ is $(k-1)$-dimensional and $g$ is one-to-one. This is a contradiction.

3. The Grassmannian manifold $M=G(k, n)$ with Lie transformation group $G=G L(n, \mathbb{C})$ satisfies the spanning property.

For simplicity we set $n=p+q, k=p ; M=G(p+q, p)$ and $G=$ $G L(p+q, \mathbb{C})$. We set $O: x_{p+1}=\ldots=x_{p+q}=0$ to be our base point of $M$. Then the isotropy sugroup $H_{0}$ of $G$ for $O$ is

$$
H_{0}=\left\{\left(\begin{array}{cc}
A_{p} & * \\
O & B_{q}
\end{array}\right) \mid \operatorname{det} A_{p}, \operatorname{det} B_{q} \neq 0\right\} .
$$

To prove 3 . it suffices to prove that $M$ satisfies the spanning property for the point $O$ and $H_{0}$. We identify $M$ as the space $G / H_{0}$ of all cosets $\left\{g H_{0}: g \in\right.$ $G$ \}. Note that $\operatorname{dim} M=p q$ and $\operatorname{dim} H_{0}=(p+q)^{2}-p q$. As local coordinates in a neighborhood of $O$ in $M$ we can take

$$
T(t)=\left(\begin{array}{c|c}
I_{p} & 0 \\
\hline(t) & I_{q}
\end{array}\right)
$$

where $(t)=\left(t_{i j}\right)$ is a $q \times p$-matrix and the base point $O$ corresponds to the identity matrix $I_{p+q}$. We identify $T(t)$ with

$$
\mathbf{t}=\left(t_{11}, t_{12}, \ldots, t_{1 p} ; \ldots ; t_{q 1}, \ldots, t_{q p}\right) \in \mathbb{C}^{p q}
$$

and we call these local coordinates the standard local coordinates at $O$. Let $M_{p+q}(\mathbb{C})$ be the set of all $(p+q)$-square matrices $X$.

Let $X \in M_{p+q}(\mathbb{C})$ satisfy condition

$$
(\star):\left.\quad \lim _{t \rightarrow 0} \frac{d \exp t X(O)}{d t}\right|_{t=0} \neq \mathbf{0} \in \mathbb{C}^{p q} .
$$

We decompose $X=A+B$ in $M_{p+q}(\mathbb{C})$ where

$$
A=\left(\begin{array}{c|c}
0 & 0 \\
\hline(a) & 0
\end{array}\right), \quad B=\left(\begin{array}{c|c}
* & * \\
\hline 0 & *
\end{array}\right),
$$


$(a)=\left(a_{i j}\right)$ in $A$ is a $q \times p$-matrix and 0 in $B$ is the $q \times p$-zero matrix. Then we have $\exp t X=\exp t(A+B)=\left(I_{p+q}+t A\right)\left(I_{p+q}+t B\right)+O\left(t^{2}\right)$. Let $h \in H_{0}$ and let $|t| \ll 1$. Since

$$
h(\exp t X)(O)=h(\exp t X) H_{0}=h\left(I_{p+q}+t A\right) h^{-1}\left[h\left(I_{p+q}+t B\right) H_{0}\right]+O\left(t^{2}\right),
$$

it follows from $h\left(I_{p+q}+t B\right) H_{0} \in H_{0}$ that

$$
h(\exp t X)(O)=h\left(I_{p+q}+t A\right) h^{-1}(O)+O\left(t^{2}\right) \quad \text { as points in } M .
$$

In particular, if we take $h=I_{p+q} \in H_{0}$, condition $(\star)$ for $X$ implies that (a) $\not \equiv 0$, i.e., there exists some $a_{\lambda \nu} \neq 0$. We show that we can choose a finite number of $h \in H_{0}$ with

$$
h\left(I_{p+q}+t A\right) h^{-1}=\left(\begin{array}{c|c}
I_{p} & 0 \\
\hline(h(t)) & I_{q}
\end{array}\right),
$$

where $(h(t))=\left(h_{i j}(t)\right)$ is a $p \times q$-holomorphic matrix in $|t| \ll 1$ and the set of tangent vectors

$$
V[h]:=\left(\begin{array}{ccc}
h_{11}^{\prime}(0), & \ldots, & h_{1 p}^{\prime}(0) \\
\vdots & \ddots & \vdots \\
h_{q 1}^{\prime}(0), & \ldots, & \left.h_{q p}^{\prime}(0)\right)
\end{array}\right)
$$

form $p q$-linearly independent vectors in $\mathbb{C}^{p q}$. Note that $h\left(I_{p+q}+t A\right) h^{-1} \in H_{0}^{\prime}$.

We first show that we may assume $a_{11} \neq 0$. We have $a_{\lambda \nu} \neq 0$ for some $1 \leq \lambda \leq p ; 1 \leq \nu \leq q$; thus we consider the following matrix $\mathbf{h}_{\nu \lambda} \in H_{0}$ :

$$
\mathbf{h}_{\nu \lambda}=\left(\begin{array}{c|c|c|c}
I_{\nu}^{*} & & \multicolumn{2}{|}{} \\
\hline & I_{p-\nu} & \multicolumn{2}{|l}{} \\
\hline & & I_{\lambda}^{*} & \\
& & I_{q-\lambda}
\end{array}\right) .
$$

Here $I_{\nu}^{*}$ is the anti-diagonal identity matrix of degree $\nu$ and the empty blocks are 0 matrices. An elementary calculation yields

$$
\mathcal{A}(t):=\mathbf{h}_{\nu \lambda}\left(I_{p+q}+t A\right) \mathbf{h}_{\nu \lambda}{ }^{-1}=\left(\begin{array}{c|c}
I_{p} & 0 \\
\hline(*) & I_{q}
\end{array}\right),
$$

where

$$
(*)=\left(\begin{array}{cccccc}
a_{\lambda \nu} t & a_{\lambda \nu-1} t & \cdots & a_{\lambda 1} t & \cdots & a_{\lambda p} t \\
\vdots & \vdots & \vdots & \vdots & \vdots & \vdots \\
a_{1 \nu} t & a_{1 \nu-1} t & \cdots & a_{11} t & \cdots & a_{1 p} t \\
\vdots & \vdots & \vdots & \vdots & \vdots & \vdots \\
a_{q \nu} t & a_{q \nu-1} t & \cdots & a_{q 1} t & \cdots & a_{q p} t
\end{array}\right)
$$


Thus we see that if our claim were true for $\mathcal{A}(t)$, then it is also true for $I_{p+q}+t A$. Hence we may assume $a_{11} \neq 0$.

We next show that we can select a finite number of $h \in H_{0}$ so that the tangent vectors $V[h]$ in (8.2) span $\mathbb{C}^{p q}$ under the condition that $a_{11} \neq 0$ in $I_{p+q}+t A$. For let $1 \leq i \leq p$ and $1 \leq j \leq q$ be fixed, and let $K \gg 1$. Consider

$$
\mathbf{h}_{i j}(K)=\left(\begin{array}{l|l|l|l}
\mathbf{m} & & \multicolumn{2}{|}{} \\
\hline & I_{p-i} & & \\
\hline & & \mathbf{n} & \\
\cline { 2 - 3 } & & I_{q-j}
\end{array}\right) \in H_{0}
$$

where $\mathbf{m}$ is an $i$-square matrix and $\mathbf{n}$ is a $j$-square matrix with

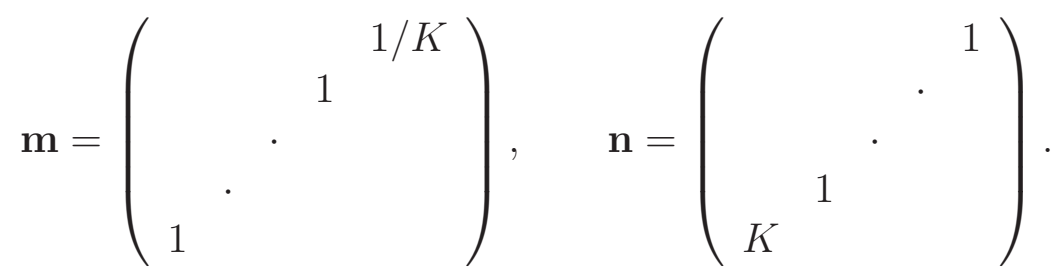

A calculation gives

$$
\mathbf{h}_{i j}(K)\left(I_{p+q}+t A\right) \mathbf{h}_{i j}(K)^{-1}=\left(\begin{array}{ccc|ccc}
1 & & & & & \\
& \ddots & & & \\
& & 1 & & \\
\hline & & & 1 & & \\
& D_{j i}(t) & & \ddots & \\
& & & & 1
\end{array}\right),
$$

where

$$
D_{j i}(t)=\left(\begin{array}{ccccccc}
a_{j i} t & a_{j i-1} t & \cdots & K a_{j 1} t & a_{j i+1} t & \cdots & a_{j p} t \\
\vdots & \vdots & \vdots & \vdots & \vdots & \vdots & \vdots \\
K a_{1 i} t & K a_{1 i-1} t & \cdots & K^{2} a_{11} t & K a_{1 i+1} t & \cdots & K a_{1 p} t \\
\vdots & \vdots & \vdots & \vdots & \vdots & \vdots & \\
a_{q i} t & a_{q i-1} t & \cdots & K a_{q 1} t & a_{q i+1} t & \cdots & a_{q p} t
\end{array}\right) .
$$

Thus the tangent vector $\mathbf{v}_{i j}:=V\left[\mathbf{h}_{i j}(K)\right]$ of the curve

$$
\mathbf{h}_{i j}(K)(I+t A) \mathbf{h}_{i j}(K)^{-1}(O)
$$


at $O$ in $K$ is

$$
\mathbf{v}_{i j}=\left(\begin{array}{ccccccc}
a_{j i} & a_{j i-1} & \cdots & K a_{j 1} & a_{j i+1} & \cdots & a_{j p} \\
\vdots & \vdots & \vdots & \vdots & \vdots & \vdots & \vdots \\
K a_{1 i} & K a_{1 i-1} & \cdots & K^{2} a_{11} & K a_{1 i+1} & \cdots & K a_{1 p} \\
\vdots & \vdots & \vdots & \vdots & \vdots & \vdots & \\
a_{q i} & a_{q i-1} & \cdots & K a_{q 1} & a_{q i+1} & \cdots & a_{q p}
\end{array}\right) \in \mathbb{C}^{p q}
$$

where $K^{2}$ occurs only in the $(j, i)$-entry. Thus if we take $K \gg 1$ sufficiently large, then $a_{11} \neq 0$ implies that $\left\{\mathbf{v}_{i j} \in \mathbb{C}^{p q}: i=1, \ldots, p ; j=1, \ldots, q\right\}$ are linearly independent in $\mathbb{C}^{p q}$ (under the identification (8.1)) and 3. is proved.

4. The flag space $M=\mathcal{F}_{n}$ for $n \geq 3$ with Lie transformation group $G=$ $G L(n, \mathbb{C})$ does not satisfy the spanning property.

We use the notation from section 7: $O:\{0\} \subset F_{1}^{0} \subset F_{2}^{0} \subset \ldots \subset F_{n-1}^{0}$ (the base point of $\mathcal{F}_{n}$ ); $H_{0}$ (the isotropy subgroup of $G$ for the point $O$ ); $\mathbf{t}=$ $\left(t_{21}, \ldots, t_{n 1} ; t_{32}, \ldots, t_{n 2} ; \ldots ; t_{n n-1}\right) \in \mathbb{C}^{n(n-1) / 2}$ where $n(n-1) / 2=\operatorname{dim} \mathcal{F}_{n}$ (the standard coordinates of a neighborhood of $O$ ). To show that $\mathcal{F}_{n}$ does not satisfy the spanning property, we take $z_{0}=O \in \mathcal{F}_{n}$ and $X=X_{21} \in$ $\mathfrak{X} \equiv M_{n}(\mathbb{C})$ such that the $(2,1)$-entry is 1 and all other entries are 0 . A calculation gives, for $t \in \mathbb{C}$,

$$
\exp t X(O): \quad\{0\} \subset F_{1}(t) \subset F_{2}(t) \subset \cdots \subset F_{n-1}(t) \subset \mathbb{C}^{n},
$$

where

$$
\begin{aligned}
& F_{1}(t): z_{2}=t z_{1}, z_{3}=\ldots=z_{n}=0 \\
& F_{j}(t): z_{j+1}=\ldots=z_{n}=0, \quad j=2, \ldots, n-1 .
\end{aligned}
$$

Fix $h \in H_{0}$. Since $h\left(F_{i}^{0}\right)=F_{i}^{0}, i=1, \ldots, n-1$, it follows that

$$
h \exp t X(O): \quad\{0\} \subset \widetilde{F}_{1}(t) \subset F_{2}^{0} \subset \ldots \subset F_{n-1}^{0} \subset \mathbb{C}^{n},
$$

where $\widetilde{F}_{1}(t)$ is a holomorphic function (depending on $h$ ) in $|t| \ll 1$. Using (7.4), this point $h \exp t X(O)$ may be written, in terms of the standard local coordinates $\mathbf{t}$, as

$$
\left(\widetilde{f}_{21}(t), \ldots, \widetilde{f}_{n 1}(t) ; 0, \ldots, 0 ; \ldots ; 0\right),
$$

where $\tilde{f}_{j 1}(t), j=2, \ldots, n$, are holomorphic functions in $|t| \ll 1$. Therefore,

$$
\left\{\left.\left[\frac{d(h \exp t X(O))}{d t}\right]\right|_{t=0} \in \mathbb{C}^{n(n-1) / 2}: h \in H_{0}\right\}
$$

contains at most $n-1$ linearly independent vectors in $\mathbb{C}^{n(n-1) / 2}$, so that $\mathcal{F}_{n}$ does not satisfy the spanning property. 


\section{Appendix B}

In this appendix, we complete the proof of Theorem 6.4. First we recall our notation. We write $H_{z_{0}}^{\prime}$ for the connected component of $H_{z_{0}}$ in $G$ containing the identity element $e$. We defined the homogeneous space $M^{\prime}:=G / H_{z_{0}}^{\prime}$ with Lie transformation group $G$. We write $w_{0}$ for the point in $M^{\prime}$ which corresponds to $H_{z_{0}}^{\prime}$ in $G / H_{z_{0}}^{\prime}$, so that the isotropy subgroup $\widehat{H}_{w_{0}}$ of $G$ for $w_{0}$ is equal to $H_{z_{0}}^{\prime}$. Since $\widehat{H}_{w_{0}}$ is a closed normal subgroup of $H_{z_{0}}$, we can consider the canonical projection $\widehat{\pi}: w=g \widehat{H}_{w_{0}} \in M^{\prime} \rightarrow z=g H_{z_{0}} \in M$, so that $\left(M^{\prime}, \widehat{\pi}\right)$ is a normal covering space over $M$. We defined $\widehat{D}=\widehat{\pi}^{-1}(D)=$ $\cup_{j=1}^{\infty} \widehat{D}_{j} \subset M^{\prime}$. Since $z_{0} \in D$, we have $w_{0} \in \widehat{\pi}^{-1}(D)$. We then focused on $\widehat{D}_{1}$, the connected component of $\widehat{\pi}^{-1}(D)$ containing $w_{0}$. We defined $\widehat{\lambda}(w):=$ $\lambda(\widehat{\pi}(w))$ for $w \in \widehat{D}_{1}$, which is a smooth plurisubharmonic exhaustion function on $\widehat{D}_{1}$.

We begin with the following.

Lemma 9.1. Let $D \Subset M$ be a domain with piecewise smooth boundary in $M$ and let $\mathcal{V}$ be a neighborhood of e in $G$. Then there exist a finite number of balls $V^{(i)}, i=1,2, \ldots, N$ centered at some point $z_{i} \in D$ such that

(1) $\bar{D} \Subset \bigcup_{i=1}^{N} V^{(i)} \Subset \mathcal{V}(D)$ where $\mathcal{V}(D)=\{g(z) \in M: g \in \mathcal{V}, z \in \bar{D}\}$;

(2) $\widehat{\pi}^{-1}\left(V^{(i)}\right)=\bigcup_{k=1}^{\infty} U_{k}^{(i)}$ (disjoint union) in $M^{\prime}$ and $\widehat{\pi}: U_{k}^{(i)} \rightarrow V^{(i)}$ is bijective;

(3) there exists a holomorphic section $\sigma_{k}^{(i)}: w \in U_{k}^{(i)} \rightarrow \sigma_{k}^{(i)}(w)$ of $G$ over $U_{k}^{(i)}$ via $\widehat{\psi}_{w_{0}}$ such that $\sigma_{i}^{(k)}\left(w^{\prime}\right)\left(\sigma_{i}^{(k)}\left(w^{\prime \prime}\right)\right)^{-1} \in \mathcal{V}$ for any $w^{\prime}, w^{\prime \prime} \in U_{i}^{(k)}$, $k=1, \ldots, N$ and $i=1,2, \ldots$

Proof. From the Borel-Lebesgue theorem it suffices to verify the following. Fix $z \in M$ and a neighborhood $\mathcal{V}$ of $e$ in $G$. We show that there exists a neighborhood $V$ of $z$ in $M$ satisfying

(1) $\widehat{\pi}^{-1}(V)=\bigcup_{k=1}^{\infty} U_{k}$ (disjoint union) in $M^{\prime}$ and $\widehat{\pi}: U_{k} \rightarrow V$ is bijective;

(2) there exists a holomorphic section $\sigma_{k}: w \in U_{k} \rightarrow \sigma_{k}(w)$ of $G$ over $U_{k}$ via $\widehat{\psi}_{w_{0}}$ such that $\sigma_{k}\left(w^{\prime}\right)\left(\sigma_{k}\left(w^{\prime \prime}\right)\right)^{-1} \in \mathcal{V}$ for any $w^{\prime}, w^{\prime \prime} \in U_{k}, k=1,2, \ldots$

To prove this, we first take $g \in G$ with $g\left(z_{0}\right)=z$. Let $v_{0}$ be the neighborhood of $e$ in $G$ stated in Proposition 6.4. Let $V \subset M, U_{k} \subset$ $M^{\prime}, \quad k=1,2, \ldots, w_{k}=g h_{k}\left(w_{0}\right)$ (the center of $\left.U_{k}\right)$ as in (6.32) and (6.31), where $\widehat{\pi}^{-1}(z)=\left\{w_{k}\right\}_{k=1,2, \ldots}$. We also want to insure that $v_{0}$ also satisfies 
$\left(g v_{0}\right)\left(g v_{0}\right)^{-1} \subset \mathcal{V}$. There exists a neighborhood $V_{0}$ of $z_{0}$ in $M$ and a holomorphic section $\sigma_{0}: \zeta \in V_{0} \rightarrow \sigma_{0}(\zeta)$ of $G$ over $V_{0}$ via $\psi_{z_{0}}$ such that $\sigma_{0}\left(z_{0}\right)=e$ and $\sigma_{0}\left(V_{0}\right) \subset v_{0}$. By redefining $v_{0}:=v_{0} \cap \pi_{z_{0}}^{-1}\left(V_{0}\right)$, we may assume that $\psi_{z_{0}}\left(v_{0}\right)=V_{0}$, so that $\sigma_{0}\left(V_{0}\right) \subset v_{0} \subset \sigma_{0}\left(V_{0}\right) H_{z_{0}}^{\prime}$, and hence $v_{0} H_{z_{0}}^{\prime}=\sigma_{0}\left(V_{0}\right) H_{z_{0}}^{\prime}$. In this situation, (6.32) implies that $V=g \sigma_{0}\left(V_{0}\right)\left(z_{0}\right)=g\left(V_{0}\right)$, and hence we have a bijection between $V$ and $V_{0}$ defined by $\zeta \in V_{0} \rightarrow \xi=g(\zeta) \in V$. Then

$$
\sigma: \xi \in V \rightarrow g \sigma_{0}(\zeta)=g \sigma_{0}\left(g^{-1}(\xi)\right) \in G
$$

is a holomorphic section of $G$ over $V$ via $\psi_{z_{0}}$ with $\sigma(z)=g \sigma_{0}\left(z_{0}\right)=g$ and $\sigma(V)=g \sigma_{0}\left(V_{0}\right)$. It follows that

$$
\begin{aligned}
U_{k} & =g v_{0} H_{z_{0}}^{\prime} h_{k}\left(w_{0}\right)=g \sigma_{0}\left(V_{0}\right) H_{z_{0}}^{\prime} h_{k}\left(w_{0}\right)=\sigma(V) H_{z_{0}}^{\prime} h_{k}\left(w_{0}\right) \\
& =\sigma(V) h_{k} H_{z_{0}}^{\prime}\left(w_{0}\right)=\sigma_{0}(V) h_{k}\left(w_{0}\right), \text { for } k=1,2, \ldots,
\end{aligned}
$$

and hence we have a bijection between $V$ and $U_{k}$ defined by $\zeta \in V \rightarrow w=$ $\sigma(\xi) h_{k}\left(w_{0}\right) \in U_{k}$ with $w_{k}=\sigma(z) h_{k}\left(w_{0}\right)=g h_{k}\left(w_{0}\right)$. Thus

$$
\sigma_{k}: w \in U_{k} \rightarrow \sigma(\xi) h_{k} \in G,
$$

is a holomorphic section of $G$ over $U_{k}$ via $\widehat{\psi}_{w_{0}}$ with $\sigma_{k}\left(w_{k}\right)=g h_{k}$. Since $\sigma_{0}(V) \subset v_{0}$, it follows from (9.1) that for $w^{\prime}, w^{\prime \prime} \in U_{k}$ there exist $\xi^{\prime}, \xi^{\prime \prime} \in V$ and $\zeta^{\prime}, \zeta^{\prime \prime} \in V_{0}$ with

$$
\begin{aligned}
\sigma_{k}\left(w^{\prime}\right) \sigma_{k}\left(w^{\prime \prime}\right)^{-1} & =\left(\sigma\left(\xi^{\prime}\right) h_{k}\right)\left(\sigma\left(\xi^{\prime \prime}\right) h_{k}\right)^{-1}=\sigma\left(\xi^{\prime}\right) \sigma\left(\xi^{\prime \prime}\right) \\
& =g \sigma_{0}\left(\zeta^{\prime}\right)\left(g \sigma_{0}\left(\zeta^{\prime \prime}\right)\right)^{-1} \in\left(g v_{0}\right)\left(g v_{0}\right)^{-1} \subset \mathcal{V},
\end{aligned}
$$

as required.

With the decomposition of $\widehat{D}$ into its connected components $\widehat{D}=\cup_{j=1}^{\infty} \widehat{D}_{j}$, where $\widehat{D}_{1}$ is the connected component containing $w_{0}$, and $D^{\prime}\left(z_{0}\right), D^{(k)}\left(z_{0}\right)$ in (6.4) are the connected components of $D\left(z_{0}\right)$ in $G$, we set $\mathbf{h}^{(k)} \in \mathcal{H}^{(k)}\left(z_{0}\right) \subset$ $D^{(k)}\left(z_{0}\right), k=2,3, \ldots$ as in equation (6.7) for $z=z_{0}$, so that $D^{(k)}\left(z_{0}\right)=$ $D^{\prime}\left(z_{0}\right) \mathbf{h}^{(k)}$. We next show that, after possibly relabeling indices,

$$
\widehat{D}_{k}=\left\{g\left(w_{0}\right) \in M^{\prime}: g \in D^{(k)}\left(z_{0}\right)\right\}, \quad k=1,2, \ldots
$$

To prove this, fix $k$ and the set $D^{(k)}\left(z_{0}\right)$. Then $\mathbf{h}^{(k)}\left(w_{0}\right) \in \widehat{\pi}^{-1}\left(z_{0}\right) \subset \widehat{\pi}^{-1}(D)$ and there exists a unique connected component of $\widehat{\pi}^{-1}(D)=\sum_{j=1}^{\infty} \widehat{D}_{j}$ which contains $\mathbf{h}^{(k)}\left(w_{0}\right)$. We call this component $\widehat{D}_{k}$ and we shall first prove equality of the two sides of (9.3) for this $k$.

We let $E_{k}$ denote the right-hand-side of (9.3). Using (6.7), we can write

$$
E_{k}=D^{\prime}\left(z_{0}\right) \mathbf{h}^{(k)}\left(w_{0}\right)=\left\{g^{\prime} \mathbf{h}^{(k)}\left(w_{0}\right) \in M^{\prime}: g^{\prime} \in D^{\prime}\left(z_{0}\right)\right\} .
$$


This is a connected set in $M^{\prime}$ which contains $\mathbf{h}^{(k)}\left(w_{0}\right)$ and satisfies $\widehat{\pi}\left(E_{k}\right) \subset D$ since $h^{(k)}\left(w_{0}\right)=z_{0}$. Hence $E_{k} \subset \widehat{D}_{k}$. To prove the reverse inclusion we set $w_{k}=\mathbf{h}^{(k)}\left(w_{0}\right) \in \widehat{D}_{k}$ and let $w \in \widehat{D}_{k}$. We take a continuous curve $\gamma: t \in$ $[0,1] \rightarrow w(t)$ in $\widehat{D}_{k}$ with $w(0)=w_{k}$ and $w(1)=w$. Then $\widehat{\pi}(\gamma): t \in[0,1] \rightarrow$ $z(t):=\widehat{\pi}(w(t))$ is a continuous curve in $D$ with $z(0)=\mathbf{h}^{(k)}\left(z_{0}\right)=z_{0}$. Using property 2. of complex homogeneous spaces from the beginning of section 6 , we can find a continuous section $\sigma: t \in[0,1] \rightarrow \sigma(t)$ in $D^{\prime}\left(z_{0}\right)$ over $\widehat{\pi}(\gamma)$ via $\psi_{z_{0}}$, i.e., $\sigma(t)\left(z_{0}\right)=z(t), t \in[0,1]$, with $\sigma(0)=e$. We set $g_{0}:=\sigma(1) \in D^{\prime}\left(z_{0}\right)$. We consider the continuous curve $\sigma \mathbf{h}^{(k)}\left(w_{0}\right): t \in[0,1] \rightarrow \sigma(t) \mathbf{h}^{(k)}\left(w_{0}\right)$ in $M^{\prime}$. Then $\widehat{\pi}(\gamma)=\widehat{\pi}\left(\sigma\left(w_{0}\right)\right) \subset D$. The two curves $\gamma$ and $\sigma \mathbf{h}^{(k)}\left(w_{0}\right)$ in $M^{\prime}$ start at the same point $w_{k}=\mathbf{h}^{(k)}\left(w_{0}\right) \in \widehat{D}_{1}$. It follows that $\gamma=\sigma \mathbf{h}^{(k)}\left(w_{0}\right)$ as curves in $M^{\prime}$, and hence $w=w(1)=\sigma(1) \mathbf{h}^{(k)}\left(w_{0}\right)=g_{0} \mathbf{h}^{(k)}\left(w_{0}\right)$. Thus $\widehat{D}_{k} \subset E_{k}$.

To finish the proof of (9.3) it remains to show that the sets $\left\{E_{k}\right\}_{k=1,2, \ldots}$ exhaust each set $\widehat{D}_{l}$. If not, by the previous argument, there exists a set $\widehat{D}_{l}$ which is not equal to any of the sets $E_{k}, k=1,2, \ldots$. From (6.6) we have $\cup_{k=1}^{\infty} \mathcal{H}_{k}\left(z_{0}\right)=H_{z_{0}}$. Since $\mathcal{H}_{k}\left(z_{0}\right) \subset D^{(k)}\left(z_{0}\right)$, we have $\left(\mathcal{H}_{k}\left(z_{0}\right)\right)\left(w_{0}\right) \subset$ $E_{k}=\widehat{D}_{k}$. Take a point $w^{*} \in \widehat{\pi}^{-1}\left(z_{0}\right) \cap \widehat{D}_{l}$. Then there exists $h^{*} \in H_{z_{0}}$ with $w^{*}=h^{*}\left(w_{0}\right)$. Since $h^{*} \in \mathcal{H}_{k}\left(z_{0}\right)$ for some $k$, we have $h^{*}\left(w_{0}\right) \in \widehat{D}_{k}$. Thus $h^{*}\left(w_{0}\right) \in \widehat{D}_{k} \cap \widehat{D}_{l}=\emptyset$, a contradiction. This proves (9.3).

Since $\widehat{D}_{1}$ is a domain in $M^{\prime}$ which contains $w_{0}$, as with our discussion of $D\left(z_{0}\right)$ we will consider the set:

$$
\widehat{D}_{1}\left(w_{0}\right):=\left\{g \in G: g\left(w_{0}\right) \in \widehat{D}_{1}\right\} \subset G .
$$

We have the following equalities:

$$
\widehat{D}_{1}=\left\{g\left(w_{0}\right) \in M^{\prime}: g \in D^{\prime}\left(z_{0}\right)\right\} ; \quad D^{\prime}\left(z_{0}\right)=\widehat{D}_{1}\left(w_{0}\right) .
$$

The first equality is the case $k=1$ in (9.3). The inclusion $D^{\prime}\left(z_{0}\right) \subset \widehat{D}_{1}\left(w_{0}\right)$ in the second equality follows from the first equality. To prove $\widehat{D}_{1}\left(w_{0}\right) \subset D^{\prime}\left(z_{0}\right)$, fix $g \in G$ with $g\left(w_{0}\right) \in \widehat{D}_{1}$. Take a continuous curve $\gamma: t \in[0,1] \rightarrow w(t) \in$ $\widehat{D}_{1}$ with $w(0)=w_{0}$ and $w(1)=g\left(w_{0}\right)$. Since $\widehat{D}_{1} \subset M^{\prime}=G / \widehat{H}_{w_{0}}$, we can find a continuous section $\sigma: t \in[0,1] \rightarrow \sigma(t)$ in $G$ over $\gamma$ via $\widehat{\psi}_{w_{0}}$ with $\sigma(0)=e$. Thus $\sigma(t)\left(w_{0}\right)=w(t), t \in[0,1]$. We set $g_{1}:=\sigma(1) \in G$. Since $g_{1}\left(w_{0}\right)=g\left(w_{0}\right)$, there exists $h \in \widehat{H}_{w_{0}}$ such that $g=g_{1} h$. Since $\widehat{H}_{w_{0}}=H_{z}^{\prime}$ is connected in $G$, we can find a continuous curve $\tau: t \in[1,2] \rightarrow \tau(t)$ in $\widehat{H}_{w_{0}}$ such that $\tau(1)=e$ and $\tau(2)=h$. Then $g_{1} \tau: t \in[1,2] \rightarrow g_{1} \tau(t)$ is a continuous curve in $G$ starting at $g_{1}$ at $t=1$ and satisfying $g_{1} \tau(t)\left(w_{0}\right)=$ $g_{1}\left(w_{0}\right)=g\left(w_{0}\right) \in \widehat{D}_{1}, t \in[1,2]\left(\right.$ since $\tau(t) \in \widehat{H}_{w_{0}}$ and $\left.\tau(t)\left(w_{0}\right)=w_{0}\right)$. We form the continuous curve $\Gamma: t \in[0,2] \rightarrow \Gamma(t)$ in $G$ via the concatenation

$$
\Gamma(t):=\sigma(t), t \in[0,1] ; \text { and } \Gamma(t):=g_{1} \tau(t), t \in[1,2] \text {. }
$$


This curve $\Gamma$ starts at $\Gamma(0)=e$ and terminates at $\Gamma(2)=g_{1} \tau(2)=g$. One can check that $\Gamma(t)\left(w_{0}\right) \in \widehat{D}_{1}, t \in[0,2]$, so that $\Gamma(t)\left(z_{0}\right) \in D, t \in[0,2]$. Since $\Gamma(0)=e \in D^{\prime}\left(z_{0}\right)$, we have $\Gamma(t) \in D^{\prime}\left(z_{0}\right), t \in[0,2]$. In particular, $g \in D^{\prime}\left(z_{0}\right)$, which verifies the inclusion $\widehat{D}_{1}\left(w_{0}\right) \subset D^{\prime}\left(z_{0}\right)$ and hence the second equality in (9.4).

Using (6.5) we set

$$
\mathcal{H}^{\prime}\left(z_{0}\right)=D^{\prime}\left(z_{0}\right) \cap H_{z_{0}}=\bigcup_{j=0}^{\infty} h_{j}^{\prime} \widehat{H}_{w_{0}} \quad \text { (disjoint union), }
$$

where $h_{1}^{\prime}=e$ and $h_{j}^{\prime} \in H_{z_{0}} \cap D^{\prime}\left(z_{0}\right), j=2,3, \ldots$ Thus, by (9.4) we have

$$
\widehat{\pi}^{-1}\left(z_{0}\right) \cap \widehat{D}_{1}=\left\{w_{0}, h_{2}^{\prime}\left(w_{0}\right), h_{3}^{\prime}\left(w_{0}\right), \ldots\right\} \equiv:\left\{w_{0}, w_{0}^{(2)}, w_{0}^{(3)}, \ldots\right\}
$$

and these points are distinct. Moreover, if $z \in D, w \in \widehat{\pi}^{-1}(z) \cap \widehat{D}_{1}$ and $g \in D^{\prime}\left(z_{0}\right)$ with $g\left(w_{0}\right)=w$ in $M^{\prime}$, then

$$
\widehat{\pi}^{-1}(z) \cap \widehat{D}_{1}=\left\{g\left(w_{0}\right), g\left(w_{0}^{(2)}\right), g\left(w_{0}^{(3)}\right), \ldots\right\} .
$$

We first verify that the right-hand side is contained in the left-hand side. Take any element $g\left(w_{0}^{(j)}\right)=g h_{j}^{\prime}\left(w_{0}\right)$ in the right-hand side. Then we have $\widehat{\pi}\left(g\left(w_{0}^{(j)}\right)\right)=g h_{j}\left(z_{0}\right)=g\left(z_{0}\right)=\widehat{\pi}\left(g\left(w_{0}\right)\right)=\widehat{\pi}(w)=z$. Since $g \in D^{\prime}\left(z_{0}\right)$ and $h_{j}^{\prime} \in D^{\prime}\left(z_{0}\right) \cap H_{z_{0}}$, we see from 2. in Proposition 6.1 that $g h_{j}^{\prime} \in D^{\prime}\left(z_{0}\right)$. Using (9.4) we have $g\left(w_{0}^{(j)}\right)=g h_{j}^{\prime}\left(w_{0}\right) \in \widehat{D}_{1}$, which proves this inclusion. To prove the reverse inclusion, let $w^{\prime} \in \widehat{D}_{1} \cap \widehat{\pi}^{-1}(z)$. Using (9.4) we can find $g^{\prime} \in D^{\prime}\left(z_{0}\right)$ with $g^{\prime}\left(w_{0}\right)=w^{\prime}$. Taking $\widehat{\pi}$ we have $g^{\prime}\left(z_{0}\right)=z=g\left(z_{0}\right)$ in $M$, so that there exists an $h \in H_{z_{0}}$ with $g^{\prime}=g h$. From 3. in Proposition 6.1 we have $h \in D^{\prime}\left(z_{0}\right) \cap H_{z}$. We thus have $h \in h_{j}^{\prime} \widehat{H}_{w_{0}}$ for some $j$, and hence $w^{\prime}=g h\left(w_{0}\right)=g h_{j}^{\prime}\left(w_{0}\right)=g\left(w_{0}^{(j)}\right)$, as claimed.

Let $E$ be a domain with piecewise smooth boundary in $M$ which contains $D$. We write $E^{\prime}\left(z_{0}\right)$ for the connected component of $E\left(z_{0}\right)$ containing $e$ in $G$. If $E^{\prime}\left(z_{0}\right) \cap H_{z_{0}}=D^{\prime}\left(z_{0}\right) \cap H_{z_{0}}=\mathcal{H}^{\prime}\left(z_{0}\right)$, then we say that $E$ and $D$ have the same isotropy class at $z_{0}$. As for $\widehat{D}_{1}$, we write $\widehat{E}_{1}$ for the connected component of $\widehat{\pi}^{-1}(E)$ containing $w_{0}$ in $M^{\prime}$.

Let $E$ be a domain containing $D$ with the same isotropy class as $D$ at $z_{0}$. Fix $z \in E$ and let $\mathcal{V}$ be a neighborhood of $e$ in $G$. Choose $g \in E^{\prime}\left(z_{0}\right)$ with $g\left(z_{0}\right)=z$. We can find a neighborhood $V$ of $z$ in $E$ which satisfies (1) and (2) in the proof of Lemma 9.1. For simplicity, if $h_{k} \in H_{z_{0}}$ is contained in $\mathcal{H}^{\prime}\left(z_{0}\right)$, say $h_{k}=h_{j}^{\prime}$, then we write $U_{j}^{\prime}=\sigma(V) h_{j}^{\prime}\left(w_{0}\right)$ for the set $U_{k}=\sigma(V) h_{k}\left(w_{0}\right)=$ $g \sigma_{0}\left(V_{0}\right) h_{k}\left(w_{0}\right)$ in (9.2). Since $g h_{j}^{\prime} \in E^{\prime}\left(z_{0}\right)$ by 2. in Proposition 6.1, it follows 
from (9.4) that the center $g h_{j}^{\prime}\left(w_{0}\right)$ of $U_{j}^{\prime}$ is contained in $\widehat{E}_{1}$. This together with $V \subset E$ implies that $U_{j}^{\prime} \subset \widehat{E}_{1} \cap \widehat{\pi}^{-1}(V)$. Moreover we show

$$
\widehat{\pi}^{-1}(V) \cap \widehat{E}_{1}=\left\{U_{1}^{\prime}=U_{1}, U_{2}^{\prime}, U_{3}^{\prime}, \ldots\right\} .
$$

It remains to prove that the left-hand side is contained in the right-hand side. Let $w^{\prime} \in \widehat{\pi}^{-1}(V) \cap \widehat{E}_{1}$. We can find $z^{\prime} \in V_{0}$ and $h_{k} \in H_{z_{0}}$ with $w^{\prime}=$ $g \sigma_{0}\left(z^{\prime}\right) h_{k}\left(w_{0}\right)$. Take a continuous curve $\gamma: t \in[0,1] \rightarrow f(t)$ in $\sigma_{0}\left(V_{0}\right)$ such that $f(0)=\sigma_{0}\left(z^{\prime}\right)$ and $f(1)=e$. Then $\widetilde{\gamma}: t \in[0,1] \rightarrow \widetilde{\gamma}(t):=g f(t) h_{k}\left(w_{0}\right)$ is a continuous curve in $M^{\prime}$ with $\widehat{\pi}(\widetilde{\gamma}(t)) \in g \sigma_{0}\left(V_{0}\right)\left(z_{0}\right)=g\left(V_{0}\right)=V \subset E$. Since $\widetilde{\gamma}(0)\left(w_{0}\right)=g \sigma_{0}\left(z^{\prime}\right) h_{k}\left(w_{0}\right)=w^{\prime} \in \widehat{E}_{1}$, it follows that $\widetilde{\gamma} \subset \widehat{E}_{1}$. In particular, we have $\widetilde{\gamma}(1)=g h_{k}\left(w_{0}\right) \in \widehat{E}_{1}$, so that $g h_{k} \in \widehat{E}_{1}\left(w_{0}\right)=E^{\prime}\left(z_{0}\right)$ by (9.4). Since $g \in E^{\prime}\left(z_{0}\right)$, this implies that $h_{k} \in \mathcal{H}^{\prime}\left(z_{0}\right)$ by 3. in Proposition 6.1, Thus (9.7) is proved.

We prove the following.

Lemma 9.2. Let $D \Subset E \Subset M$ and $z_{0} \in D$. Let $t \in[0,1] \rightarrow D(t) \Subset M$ be a one-parameter family of domains $D(t)$ satisfying $D \subset D(t) \subset E ; D(0)=$ $D ; D(1)=E$; and each boundary $\partial D(t)$ is piecewise smooth in $M$ with $\partial D(t)$ varying continuously with $t \in[0,1]$. Then

(i) $D$ and $E$ have the same isotropy class at $z_{0}$;

(ii) $\widehat{D}_{1}=\widehat{E}_{1} \cap \widehat{\pi}^{-1}(D)$ in $M^{\prime}$;

(iii) $D^{\prime}\left(z_{0}\right)=E^{\prime}\left(z_{0}\right) \cap D\left(z_{0}\right)$ in $G$.

Proof. Let $D \Subset M$ be a domain with piecewise smooth boundary $\partial D$. Let $z_{0} \in D$. Fix $z \in \partial D$. We choose a neighborhood $V=g\left(V_{0}\right)$ of $z$ in $M$ as constructed in the proof of Lemma 9.1 and we set $E:=D \cup V \subset M$. To prove Lemma 9.2 it suffices to verify (i), (ii) and (iii) for such $D$ and $E$. To prove (i), i. e.,

$$
E^{\prime}\left(z_{0}\right) \cap H_{z_{0}}=D^{\prime}\left(z_{0}\right) \cap H_{z_{0}},
$$

we consider $\widetilde{D}_{1}:=\widehat{\pi}^{-1}(D) \cap \widehat{E}_{1} \subset M^{\prime}$. Thus, if $g \in G$ with $g\left(w_{0}\right) \in \widetilde{D}_{1}$, then $g\left(z_{0}\right) \in D$. Using (6.33), we have

$$
\widehat{E}_{1} \cap \widehat{\pi}^{-1}(V)=\bigcup_{j=1}^{\infty} U_{k_{j}},
$$

where the disjoint union $\left\{U_{k_{j}}\right\}_{j}$ is a subsequence of $\left\{U_{k}\right\}_{k}$ in (6.33). To verify (9.8) it suffices to show that $E^{\prime}\left(z_{0}\right) \cap H_{z} \subset D^{\prime}\left(z_{0}\right)$. Fix $h \in E^{\prime}\left(z_{0}\right) \cap H_{z}$. To show that $h \in D^{\prime}\left(z_{0}\right)$, fix a continuous curve $\tau: t \in[0,1] \rightarrow \tau(t)$ in $E^{\prime}\left(z_{0}\right)$ 
with $\tau(0)=e$ and $\tau(1)=h$. Using (9.4) for the set $E$, the curve $\gamma:=\tau\left(w_{0}\right)$ defined by $t \in[0,1] \rightarrow w(t):=\tau(t)\left(w_{0}\right)$ is a continuous curve in $\widehat{E}_{1}$ with $w(0)=w_{0}$ and $w(1)=h\left(w_{0}\right)$. We can take a partition $0=t_{1}<t_{2}<\ldots<$ $t_{2 \mu-1}<t_{2 \mu}=1$ of $[0,1]$ such that

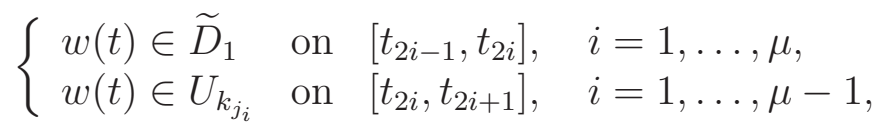

where each set $U_{k_{j_{i}}}$ is one of the sets in the disjoint union $\left\{U_{k_{j}}\right\}_{j=1,2, \ldots}$. For simplicity in notation we write $I_{i}:=\left[t_{2 i}, t_{2 i+1}\right]$ and $U_{i}:=U_{k_{j_{i}}}$ for $i=1, \ldots, \mu-1$. For $i=1, \ldots, \mu-1$, if we set $\gamma_{2 i}: t \in I_{i} \rightarrow w(t)$, then $\gamma_{2 i}$ is a continuous curve in $U_{i}$ with $w\left(t_{2 i}\right), w\left(t_{2 i+1}\right) \in \widetilde{D}_{1}$. For each $i$, we will construct a continuous curve $\widetilde{\tau}_{2 i}: t \in I_{i} \rightarrow \widetilde{\tau}_{2 i}(t)$ in $G$ such that $\widetilde{\tau}_{2 i}(t)\left(w_{0}\right) \in \widetilde{D}_{1}$ in $M^{\prime}$ and $\widetilde{\tau}_{2 i}\left(t_{2 i}\right)=\tau\left(t_{2 i}\right), \widetilde{\tau}_{2 i}\left(t_{2 i+1}\right)=\tau\left(t_{2 i+1}\right)$.

We first consider a continuous curve $\widetilde{\gamma}_{2 i}: t \in I_{i} \rightarrow \widetilde{w}(t)$ in $\widetilde{D}_{1} \cap U_{i}$ with $\widetilde{w}\left(t_{2 i}\right)=w\left(t_{2 i}\right), \widetilde{w}\left(t_{2 i+1}\right)=w\left(t_{2 i+1}\right)$. From the proof of Lemma 9.1, there is a holomorphic section $\sigma_{i}: w \in U_{i} \rightarrow \sigma_{i}(w)$ of $G$ over $U_{i}$ via $\widehat{\psi}_{w_{0}}$, i.e., $\sigma_{i}(w)\left(w_{0}\right)=w, w \in U_{i}$. Thus, for $t \in I_{i}, \sigma_{i}(w(t))\left(w_{0}\right)=w(t)=\tau(t)\left(w_{0}\right)$ in $M^{\prime}$. Hence we can find a continuous curve $t \in I_{i} \rightarrow h(t)$ in $\widehat{H}_{w_{0}}$ with $\tau(t)=\sigma_{i}(w(t)) h(t), t \in I_{i}$. Next we consider the continuous curve

$$
\widetilde{\tau}_{2 i}: t \in I_{i} \rightarrow \widetilde{\tau}_{2 i}(t):=\sigma_{i}(\widetilde{w}(t)) h(t)
$$

in $G$. Then we have $\widetilde{\tau}_{2 i}(t)\left(w_{0}\right)=\sigma_{i}(\widetilde{w}(t))\left(w_{0}\right)=\widetilde{w}(t) \in \widetilde{D}_{1}$, and

$$
\widetilde{\tau}_{2 i}\left(t_{2 i}\right)=\sigma_{i}\left(\widetilde{w}\left(t_{2 i}\right)\right) h\left(t_{2 i}\right)=\sigma_{i}\left(w\left(t_{2 i}\right)\right) h\left(t_{2 i}\right)=\tau\left(t_{2 i}\right) ;
$$

similarly $\widetilde{\tau}_{2 i}\left(t_{2 i+1}\right)=\tau\left(t_{2 i+1}\right)$. Thus $\widetilde{\tau}_{2 i}$ satisfies the desired properties.

Now for $i=1, \ldots, \mu$, let $\tau_{2 i-1}$ denote the restriction of $\tau$ to the subinterval $\left[t_{2 i-1}, t_{2 i}\right]$. Consider the concatenized curve

$$
T:=\tau_{1}+\widetilde{\tau}_{2}+\tau_{3}+\cdots+\widetilde{\tau}_{2 \mu-2}+\tau_{2 \mu-1}
$$

in $G$. Clearly $T$ is continuous on $[0,1]$ and $T(t)\left(w_{0}\right) \in \widetilde{D}_{1}$. Hence $T(t)\left(z_{0}\right) \in$ $D, t \in[0,1]$. Since $T(0)=e$, it follows from the definition of $D^{\prime}\left(z_{0}\right)$ that $T(t) \in D^{\prime}\left(z_{0}\right)$. In particular, $h=\tau(1)=T(1) \in D^{\prime}\left(z_{0}\right)$, which proves (i).

To prove (ii), note first that it is clear that the left-hand-side is contained in the right-hand-side. To prove the reverse inclusion, let $w \in \widehat{\pi}^{-1}(D) \cap \widehat{E}_{1}$, so that $z:=\widehat{\pi}(w) \in D$, and take $g \in E^{\prime}\left(z_{0}\right)=\widehat{E}_{1}\left(w_{0}\right)$ with $g\left(w_{0}\right)=w$. Thus $\widehat{\pi}\left(g\left(w_{0}\right)\right)=\widehat{\pi}(w)$; i.e., we have $g\left(z_{0}\right)=z$ in $M$. Using 1 . in Proposition 6.1 we can find $g^{\prime} \in D^{\prime}\left(z_{0}\right)$ such that $g^{\prime}\left(z_{0}\right)=z$. Thus there exists $h \in H_{z_{0}}$ with $g=g^{\prime} h$ in $G$. Since $g \in E^{\prime}\left(z_{0}\right)$ and $g^{\prime} \in D^{\prime}\left(z_{0}\right) \subset E^{\prime}\left(z_{0}\right)$, we apply 
3. in Proposition 6.1 to obtain $h \in E^{\prime}\left(z_{0}\right) \cap H_{z_{0}}$, so that $h \in D^{\prime}\left(z_{0}\right) \cap H_{z_{0}}$ by (i). It follows from 2. in Proposition 6.1 that $g=g^{\prime} h \in D^{\prime}\left(z_{0}\right)$. Hence $w=g\left(w_{0}\right) \in \widehat{D}_{1}$ by (9.4), which proves the reverse inclusion in (ii).

For (iii), again, that the left-hand-side is contained in the right-hand-side is clear. To prove the reverse inclusion, let $g \in E^{\prime}\left(z_{0}\right) \cap D\left(z_{0}\right)$. By (9.4) we have $w:=g\left(w_{0}\right) \in \widehat{E}_{1}$. We set $z:=g\left(z_{0}\right) \in D$. Since $\widehat{\pi}(w)=g\left(z_{0}\right)=z$, it follows that $w \in \widehat{E}_{1} \cap \widehat{\pi}^{-1}(z)$, so that $w \in \widehat{D}_{1}$ by (ii). Again using (9.4) we can find $g^{\prime} \in D^{\prime}\left(z_{0}\right)$ such that $w=g^{\prime}\left(w_{0}\right)$, and hence there exists $h \in H_{z_{0}}^{\prime}$ with $g=g^{\prime} h$. Therefore, 2. in Proposition 6.1 implies that $g \in D^{\prime}\left(z_{0}\right)$, which proves the reverse inclusion in (iii).

For the proof of Theorem 6.4 we next study the subset $\Sigma_{z_{0}} \mathcal{H}^{\prime}\left(z_{0}\right)$ of $G$ defined in (6.37).

\section{Lemma 9.3.}

1. If $h \in \mathcal{H}^{\prime}\left(z_{0}\right)$, then $h^{-1} \Sigma_{z_{0}} h=\Sigma_{z_{0}}$.

2. The set $\Sigma_{z_{0}} \mathcal{H}^{\prime}\left(z_{0}\right)$ is a Lie subgroup of $G$ such that

(i) $\Sigma_{z_{0}}$ is a normal Lie subgroup of $\Sigma_{z_{0}} \mathcal{H}^{\prime}\left(z_{0}\right)$;

(ii) $\Sigma_{z_{0}} \mathcal{H}^{\prime}\left(z_{0}\right)=\mathcal{H}^{\prime}\left(z_{0}\right) \Sigma_{z_{0}}$ and $D^{\prime}\left(z_{0}\right) \Sigma_{z_{0}} \mathcal{H}^{\prime}\left(z_{0}\right)=D^{\prime}\left(z_{0}\right)$;

(iii) using the notation $h_{j}^{\prime}, j=1,2, \ldots$ in (6.5), we have

$$
\Sigma_{z_{0}} \mathcal{H}^{\prime}\left(z_{0}\right)=\bigcup_{j=1}^{\infty} h_{j}^{\prime} \Sigma_{z_{0}}=\bigcup_{j=1}^{\infty} \Sigma_{z_{0}} h_{j}^{\prime}
$$

and these are disjoint unions;

(iv) $\Sigma_{z_{0}} \mathcal{H}^{\prime}\left(z_{0}\right)=D^{\prime}\left(z_{0}\right) \cap$ Aut $\sigma_{z_{0}}$, which acts transitively on $\sigma_{z_{0}}$; and $\sigma_{z_{0}}$ is isomorphic to $\Sigma_{z_{0}} \mathcal{H}^{\prime}\left(z_{0}\right) / \mathcal{H}^{\prime}\left(z_{0}\right)$ (as complex manifolds).

3. If $\sigma_{z_{0}}$ is closed in $M$, then $\Sigma_{z_{0}} \mathcal{H}^{\prime}\left(z_{0}\right)$ is a closed Lie subgroup of $G$.

Proof. We prove 1. and 2. in the lemma in steps $(1) \sim(6)$.

(1) Each of $\Sigma_{z_{0}} \mathcal{H}^{\prime}\left(z_{0}\right)$ and $\mathcal{H}^{\prime}\left(z_{0}\right) \Sigma_{z_{0}}$ are contained in $D^{\prime}\left(z_{0}\right)$.

To see this, let $s \in \Sigma_{z_{0}}$ and $h \in \mathcal{H}^{\prime}\left(z_{0}\right)$. To prove that $s h \in D^{\prime}\left(z_{0}\right)$ we take a continuous curve $\gamma: t \in[0,1] \rightarrow s(t)$ in $\Sigma_{z_{0}}$ with $s(0)=e$ and $s(1)=s$ (note that $\Sigma_{z_{0}}$ is connected and contains $e$ ). Then $\widetilde{\gamma}: t \in[0,1] \rightarrow \widetilde{\gamma}(t)=$ $s(t) h$ is a continuous curve in $G$. We have

$$
\widetilde{\gamma}(t)\left(z_{0}\right)=s(t) h\left(z_{0}\right)=s(t)\left(z_{0}\right) \in \Sigma_{z_{0}}\left(z_{0}\right)=\sigma_{z_{0}} \Subset D, \quad t \in[0,1] .
$$

Since $\widetilde{\gamma}(0)=h \in \mathcal{H}^{\prime}\left(z_{0}\right) \subset D^{\prime}\left(z_{0}\right)$, it follows that $\widetilde{\gamma}\left(z_{0}\right) \subset D^{\prime}\left(z_{0}\right)$. Hence sh $=\widetilde{\gamma}(1) \in D^{\prime}\left(z_{0}\right)$, so that $\Sigma_{z_{0}} \mathcal{H}^{\prime}\left(z_{0}\right) \subset D^{\prime}\left(z_{0}\right)$. 
To verify the inclusion $\mathcal{H}^{\prime}\left(z_{0}\right) \Sigma_{z_{0}} \subset D^{\prime}\left(z_{0}\right)$, let $h \in \mathcal{H}^{\prime}\left(z_{0}\right)$ and $s \in \Sigma_{z_{0}}$. We take the same continuous curve $\gamma: t \in[0,1] \rightarrow s(t)$ in $\Sigma_{z_{0}}$ with $s(0)=e$ and $s(1)=s$; then $\widehat{\gamma}: t \in[0,1] \rightarrow \widehat{\gamma}(t)=h s(t)$ is a continuous curve in $G$.We then have

$$
\widehat{\gamma}(t)\left(z_{0}\right)=h s(t)\left(z_{0}\right) \in h \sigma_{z_{0}} \Subset D, \quad t \in[0,1] .
$$

The last equality comes from $h \in D^{\prime}\left(z_{0}\right)$ and formula (6.17). Since $\widehat{\gamma}(0)=$ $h \in D^{\prime}\left(z_{0}\right)$, we have $h s=\widehat{\gamma}(1) \in D^{\prime}\left(z_{0}\right)$. Thus (1) is proved.

(2) If $h \in \mathcal{H}^{\prime}\left(z_{0}\right)$, then $h^{-1} \Sigma_{z_{0}} h=\Sigma_{z_{0}}$. In particular, $\mathcal{H}^{\prime}\left(z_{0}\right) \Sigma_{z_{0}}=$ $\Sigma_{z_{0}} \mathcal{H}^{\prime}\left(z_{0}\right)$.

Let $\Sigma^{\prime}:=h^{-1} \Sigma_{z_{0}} h$. Then $\Sigma_{z_{0}}$ and $\Sigma^{\prime}$ are Lie subgroups of $G$ of the same dimension. Since $H_{z_{0}}^{\prime}$ is normal in $H_{z_{0}}, \Sigma^{\prime}$ and $\Sigma_{z_{0}}$ each contain $H_{z_{0}}^{\prime}$, which is of dimension $m_{0}$. We write

$$
\mathfrak{h}_{0}=\left[X_{1}, \ldots, X_{m_{0}}\right] \text { and } \mathfrak{X}^{\prime}=\left[X_{1}, \ldots, X_{m_{0}}, X_{m_{0}+1}, \ldots, X_{m_{0}+m_{1}}\right]
$$

for the Lie algebras of $H_{z_{0}}^{\prime}$ and $\Sigma^{\prime}$. Thus

$$
\Sigma^{\prime}=\left\{\prod_{i=1}^{\nu} \exp t_{i} X_{i} \in G: \nu \in \boldsymbol{Z}^{+}, t_{i} \in \mathbb{C}, X_{i} \in \mathfrak{X}^{\prime}\right\} .
$$

To verify (2), it suffices to prove that $\mathfrak{X}^{\prime} \subset \mathfrak{X}_{z_{0}}$, or equivalently,

$$
X_{j} \in \mathfrak{X}_{z_{0}}, \quad j=m_{0}+1, \ldots, m_{0}+m_{1} .
$$

Fixing such a $j$, we consider the one-dimensional curve $C:=\left\{\exp t X_{j}\left(z_{0}\right) \in\right.$ $M: t \in \mathbb{C}\}$ in $M$. Since $\exp t X_{j} \in \Sigma^{\prime}$ and $h^{-1} \in \mathcal{H}\left(z_{0}{ }^{\prime}\right) \subset D^{\prime}\left(z_{0}\right)$, we have

$$
C \subset \Sigma^{\prime}\left(z_{0}\right)=h^{-1} \Sigma_{z_{0}} h\left(z_{0}\right)=h^{-1} \Sigma_{z_{0}}\left(z_{0}\right)=h^{-1} \sigma_{z_{0}} \Subset D .
$$

It follows that $\lambda=$ const. $=\lambda\left(z_{0}\right)$ on $C$, and hence $X_{j} \in \mathfrak{X}_{z_{0}}$ from the definition of $\mathfrak{X}_{z_{0}}$. This proves (2).

(3) $D^{\prime}\left(z_{0}\right) \Sigma_{z_{0}} \mathcal{H}^{\prime}\left(z_{0}\right)=D^{\prime}\left(z_{0}\right)$.

The inclusion $D^{\prime}\left(z_{0}\right) \subset D^{\prime}\left(z_{0}\right) \Sigma_{z_{0}} \mathcal{H}^{\prime}\left(z_{0}\right)$ is clear; we prove the reverse inclusion. Let $g \in D^{\prime}\left(z_{0}\right), s \in \Sigma_{z_{0}}, h \in \mathcal{H}^{\prime}\left(z_{0}\right)$. Since $\Sigma_{z_{0}}$ is connected and contains $e$ and $s$, we can find a continuous curve $l: t \in[0,1] \rightarrow l(t):=g s(t)$ in $G$ with $s(t) \in \Sigma_{z_{0}}, t \in[0,1] ; l(0)=g$ and $l(1)=g s$. Since $l(0)=g \in D^{\prime}\left(z_{0}\right)$, we have $l \subset D^{\prime}\left(z_{0}\right)$, and hence $g s=l(1) \in D^{\prime}\left(z_{0}\right)$. Since $h \in \mathcal{H}^{\prime}\left(z_{0}\right)=$ $D^{\prime}\left(z_{0}\right) \cap H_{z_{0}}$, it follows from 2. in Proposition 6.1 that $g s h \in D^{\prime}\left(z_{0}\right)$, as required.

(4) We have 2. (iii) in the lemma. 
From (6.5) we have

$$
\begin{array}{rlr}
\Sigma_{z_{0}} \mathcal{H}^{\prime}\left(z_{0}\right) & =\Sigma_{z_{0}} \bigcup_{j=1}^{\infty} h_{j}^{\prime} H_{z_{0}}^{\prime} & \\
& =\bigcup_{j=1}^{\infty} \Sigma_{z_{0}} h_{j}^{\prime} H_{z_{0}}^{\prime} & \\
& =\bigcup_{j=1}^{\infty} h_{j}^{\prime} \Sigma_{z_{0}} H_{z_{0}}^{\prime} & (\text { by }(2)) \\
& =\bigcup_{j=1}^{\infty} h_{j}^{\prime} \Sigma_{z_{0}} &
\end{array}
$$

To prove that this is a disjoint union, assume that $h_{j}^{\prime} \Sigma_{z_{0}} \cap h_{k}^{\prime} \Sigma_{z_{0}} \neq \emptyset$. Then $h_{j}^{\prime} s_{j}=h_{k}^{\prime} s_{k}$ for some $s_{j}, s_{k} \in \Sigma_{z_{0}}$. If we define $\alpha:=\left(h_{k}^{\prime}\right)^{-1} h_{j}^{\prime}=s_{k}\left(s_{j}\right)^{-1}$, then $\alpha \in \mathcal{H}^{\prime}\left(z_{0}\right) \cap \Sigma_{z_{0}}$, since both $\mathcal{H}^{\prime}\left(z_{0}\right)$ and $\Sigma_{z_{0}}$ are subgroups in $G$. It follows that $h_{j}^{\prime} \Sigma_{z_{0}}=h_{k}^{\prime} \alpha \Sigma_{z_{0}} \in h_{k}^{\prime} \Sigma_{z_{0}}$, and hence $h_{j}^{\prime} \Sigma_{z_{0}}=h_{k}^{\prime} \Sigma_{z_{0}}$, and (4) is proved.

(5) The set $\Sigma_{z_{0}} \mathcal{H}^{\prime}\left(z_{0}\right)$ is a Lie subgroup of $G$, and $\Sigma_{z_{0}}$ is a normal Lie subgroup of $\Sigma_{z_{0}} \mathcal{H}^{\prime}\left(z_{0}\right)$.

By (4) the set $\Sigma_{z_{0}} \mathcal{H}^{\prime}\left(z_{0}\right)$ is a disjoint union of $\left(m_{0}+m_{1}\right)$-dimensional nonsingular $f$-generalized analytic sets which are equivalent to $\Sigma_{z_{0}}$. Thus we need only verify that $\Sigma_{z_{0}} \mathcal{H}^{\prime}\left(z_{0}\right)$ is a subgroup of $G$. Since $e \in \Sigma_{z_{0}} \mathcal{H}^{\prime}\left(z_{0}\right)$, it suffices to prove that for any $s_{1}, s_{2} \in \Sigma_{z_{0}}$ and $h_{1}, h_{2} \in \mathcal{H}^{\prime}\left(z_{0}\right)$, we have $x:=\left(s_{1} h_{1}\right)\left(s_{2} h_{2}\right)^{-1} \in \Sigma_{z_{0}} \mathcal{H}^{\prime}\left(z_{0}\right)$. To see this,

$$
\begin{aligned}
& x=s_{1} h_{1} h_{2}^{-1} s_{2}^{-1} \\
& \in \Sigma_{z_{0}} \mathcal{H}^{\prime}\left(z_{0}\right) \Sigma_{z_{0}} \quad \text { (since } \mathcal{H}^{\prime}\left(z_{0}\right) \text { is a group) } \\
& \subset \Sigma_{z_{0}} \Sigma_{z_{0}} \mathcal{H}^{\prime}\left(z_{0}\right) \quad(\text { by }(2)) \\
& =\Sigma_{z_{0}} \mathcal{H}^{\prime}\left(z_{0}\right) \quad \text { (since } \Sigma_{z_{0}} \text { is a group), }
\end{aligned}
$$

so that $\Sigma_{z_{0}} \mathcal{H}^{\prime}\left(z_{0}\right)$ is a Lie subgroup of $G$. This with (2) implies that $\Sigma_{z_{0}}$ is a normal Lie subgroup of $\Sigma_{z_{0}} \mathcal{H}^{\prime}\left(z_{0}\right)$.

(6) We have 2. (iv) in the lemma.

For the first equality in (iv) it suffices to show that

$$
\Sigma_{z_{0}} \mathcal{H}^{\prime}\left(z_{0}\right)=D^{\prime}\left(z_{0}\right) \cap \text { Aut } \sigma_{z_{0}} .
$$

Let $g=s h \in \Sigma_{z_{0}} \mathcal{H}^{\prime}\left(z_{0}\right)$. Then $g \sigma_{z_{0}}=s h \sigma_{z_{0}}=\operatorname{sh} \Sigma_{z_{0}}\left(z_{0}\right)=s \Sigma_{z_{0}} h\left(z_{0}\right)=$ $\Sigma_{z_{0}}\left(z_{0}\right)=\sigma_{z_{0}}$. This together with (3) proves $\Sigma_{z_{0}} \mathcal{H}^{\prime}\left(z_{0}\right)=\subset D^{\prime}\left(z_{0}\right) \cap$ Aut $\sigma_{z_{0}}$. For the reverse inclusion, let $g \in D^{\prime}\left(z_{0}\right)$ with $g \sigma_{z_{0}}=\sigma_{z_{0}}$. Then $g\left(z_{0}\right)=s\left(z_{0}\right)$ for some $s \in \Sigma_{z_{0}}$, so that $g=s h$ for some $h \in H_{z_{0}}$. Since $g, s \in D^{\prime}\left(z_{0}\right)$, we have $h \in \mathcal{H}^{\prime}\left(z_{0}\right)$ by 3 . in Proposition 6.1. Hence $g \in \Sigma_{z_{0}} \mathcal{H}^{\prime}\left(z_{0}\right)$, as claimed. To prove the transitivity of $\Sigma_{z_{0}} \mathcal{H}^{\prime}\left(z_{0}\right)$ on $\sigma_{z_{0}}$, let $\zeta \in \sigma_{z_{0}}$; there exists $s \in \Sigma_{z_{0}}$ with $\zeta=s\left(z_{0}\right)$. Since $\sigma_{z_{0}} \subset D$, we have $g \in D^{\prime}\left(z_{0}\right)$ with $g\left(z_{0}\right)=\zeta$, hence we can find $h \in H_{z_{0}}$ with $g=s h$; again, this implies $h \in \mathcal{H}^{\prime}\left(z_{0}\right)$. 
Finally to show $\Sigma_{z_{0}} \mathcal{H}^{\prime}\left(z_{0}\right) / \mathcal{H}^{\prime}\left(z_{0}\right) \approx \sigma_{z_{0}}$, it suffices to prove that the isotropy subgroup $\left\{g \in \Sigma_{z_{0}} \mathcal{H}^{\prime}\left(z_{0}\right): g\left(z_{0}\right)=z_{0}\right\}$ of $\Sigma_{z_{0}} \mathcal{H}^{\prime}\left(z_{0}\right)$ for $z_{0} \in \sigma_{z_{0}}$ is equal to $\mathcal{H}^{\prime}\left(z_{0}\right)$. This follows from the definition $\mathcal{H}^{\prime}\left(z_{0}\right)=H_{z_{0}} \cap D^{\prime}\left(z_{0}\right)$ and (3).

This completes the proofs of $(1) \sim(6)$, and hence 1 . and 2 . in the lemma are proved. To prove 3. we assume that $\sigma_{z}$ is closed in $M$. Let $s_{n} h_{n} \in \Sigma_{z} \mathcal{H}^{\prime}(z), n=1,2, \ldots$, and take $g \in G$ with $s_{n} h_{n} \rightarrow g$ as $n \rightarrow \infty$. Since $s_{n} h_{n}(z)=s_{n}(z) \in \Sigma_{z}(z)=\sigma_{z} \Subset D$, and since $\sigma_{z}$ is assumed to be closed in $G$, the limit $g(z)=\lim _{n \rightarrow \infty} s_{n} h_{n}(z)$ is contained in $\sigma_{z}$. Here, if necessary, we take a subsequence of $\left\{s_{n} h_{n}(z)\right\}_{n}$. Thus there exists $s^{*} \in \Sigma_{z}$ with $g(z)=s^{*}(z)$. By definition of the isotropy subgroup $H_{z}$, there exsists $h^{*} \in H_{z}$ such that $g=s^{*} h^{*}$. Since $g(z) \in D$, we have $g \notin \partial[D(z)]$. On the other hand, by (1) we have $s_{n} h_{n} \in D^{\prime}(z)$, so that $g \in D^{\prime}(z) \cup \partial\left[D^{\prime}(z)\right]$. Using $\partial\left[D^{\prime}(z)\right] \subset \partial[D(z)]$, we have $g \in D^{\prime}(z)$. Moreover, we have $s^{*} \in \Sigma_{z} \subset D^{\prime}(z)$. Applying 3. in Proposition 6.1 to $g=s^{*} h^{*}$, we have $h^{*} \in \mathcal{H}^{\prime}(z)$. Hence $g \in \Sigma_{z} \mathcal{H}^{\prime}(z)$, and 3 . in the lemma is proved.

From 2. (ii) in Lemma 9.3 we can show that the union stated in (6.17):

$$
D=\bigcup_{g \in D} g \sigma_{z_{0}} \text { is a disjoint union. }
$$

To see this, take $s_{1}, s_{2} \in \Sigma_{z_{0}}$ with $g_{1} s_{1}\left(z_{0}\right)=g_{2} s_{2}\left(z_{0}\right)$. Then we can find $h \in H_{z_{0}}$ with $g_{1} s_{1}=g_{2} s_{2} h$. From 2. (ii) in Lemma 6.1 we have $g_{1} s_{1}, g_{2} s_{2} \in$ $D^{\prime}\left(z_{0}\right)$; then from 3. in Proposition 6.1 we have $h \in \mathcal{H}^{\prime}\left(z_{0}\right)$. Next, from 1 . in Lemma 9.3 we have $g_{1} s_{1}=g_{2} h s_{2}^{\prime}$ for some $s_{2}^{\prime} \in \Sigma_{z_{0}}$. Since $\Sigma_{z_{0}}$ is a group, it follows from 1 . in Lemma 9.3 that

$$
\begin{aligned}
g_{1} \sigma_{z_{0}} & =g_{1} \Sigma_{z_{0}}\left(z_{0}\right)=g_{1} s_{1} \Sigma_{z_{0}}\left(z_{0}\right)=g_{2} h s_{2}^{\prime} \Sigma_{z_{0}}\left(z_{0}\right) \\
& =g_{2} h \Sigma_{z_{0}}\left(z_{0}\right)=g_{2} \Sigma_{z_{0}} h\left(z_{0}\right)=g_{2} \Sigma_{z_{0}}\left(z_{0}\right)=g_{2} \sigma_{z_{0}},
\end{aligned}
$$

as claimed.

Recall the notation $\hat{\lambda}(w)$ and $\widehat{\Sigma}_{w_{0}}$ in (6.34) and (6.35):

$$
\begin{aligned}
& \widehat{\lambda}(w)=\lambda(\widehat{\pi}(w)), \quad w \in \widehat{D}_{1} ; \\
& \widehat{\mathfrak{X}}_{w_{0}}=\left\{X \in \mathfrak{X}:\left.\left[\frac{\partial^{2} \widehat{\lambda}\left(\exp t X\left(w_{0}\right)\right)}{\partial t \partial \bar{t}}\right]\right|_{t=0}=0\right\} ; \\
& \widehat{\Sigma}_{w_{0}}=\left\{\prod_{i=1}^{\nu} \exp t_{i} X_{i} \in G: \nu \in \boldsymbol{Z}^{+}, t_{i} \in \mathbb{C}, X_{i} \in \widehat{\mathfrak{X}}_{w_{0}}\right\} .
\end{aligned}
$$

Lemma 9.4. With the above notation,

(1) $\widehat{\mathfrak{X}}_{w_{0}}=\mathfrak{X}_{z_{0}}$ and hence $\Sigma_{z_{0}}=\widehat{\Sigma}_{w_{0}}$;

(2) $\widehat{\mathfrak{X}}_{w_{0}}=\left\{X \in \mathfrak{X}: \exp t X\left(w_{0}\right) \in \widehat{D}_{1}, \widehat{\lambda}\left(\exp t X\left(w_{0}\right)\right)=\widehat{\lambda}\left(w_{0}\right), t \in \mathbb{C}\right\}$. 
Proof. If $|t| \ll 1$, then $\exp t X\left(w_{0}\right) \in \widehat{D}_{1}$ and $\exp t X\left(z_{0}\right) \in D$. From the definition of $\widehat{\lambda}$ and $\widehat{\pi}$ and (6.30), we have $\hat{\lambda}\left(\exp t X\left(w_{0}\right)\right)=\lambda\left(\exp t X\left(z_{0}\right)\right)$. Thus (1) follows from the definition of the sets $\widehat{\mathfrak{X}}_{w_{0}}$ and $\mathfrak{X}_{z_{0}}$. We write $\widetilde{\mathfrak{X}}_{w_{0}}$ for the right-hand side of (2). Let $X \in \widehat{\mathfrak{X}}_{w_{0}}$. By (1) we have $X \in \mathfrak{X}_{z_{0}}$, so that, in particular, $X$ satisfies condition i. in the definition of $\mathfrak{X}_{z_{0}}$ in (6.11). It follows from the definitions of $\widehat{D}_{1}$ and $\widehat{\lambda}$ that $X \in \widetilde{\mathfrak{X}}_{w_{0}}$, so that $\widehat{\mathfrak{X}}_{w_{0}} \subset \widetilde{\mathfrak{X}}_{w_{0}}$. The reverse inclusion is clear, and (2) is proved.

Using standard arguments this lemma implies the following results. From (1) in Lemma 9.4, $\widehat{\mathfrak{X}}_{w_{0}}$ is a Lie subalgebra of $\mathfrak{X}$ with $\widehat{H}_{w_{0}}=H_{z_{0}}^{\prime} \subsetneq \mathfrak{X}_{z_{0}}=\widehat{\mathfrak{X}}_{w_{0}}$. Now we let $\widehat{\mathfrak{h}}_{w_{0}}$ denote the Lie subalgebra of $\mathfrak{X}$ which corresponds to the closed Lie subgroup $\widehat{H}_{w_{0}}$ of $G$. Note that $\operatorname{dim} \widehat{\mathfrak{h}}_{w_{0}}=m_{0}=\operatorname{dim} H_{z_{0}}$, and let $\operatorname{dim} \widehat{\mathfrak{X}}_{w_{0}}=m_{0}+m_{1}=\operatorname{dim} \mathfrak{X}_{z_{0}}$. Recall we used the notation $\widehat{\Sigma}_{w_{0}}$ for the connected Lie subgroup of $G$ which corresponds to $\widehat{\mathfrak{X}}_{w_{0}}$, even though this is the same as $\Sigma_{z_{0}}$. From the Frobenius theorem, $\widehat{\Sigma}_{w_{0}}$ is an irreducible, $\left(m_{0}+m_{1}\right)$-dimensional non-singular $f$-generalized analytic set in $G$. Define

$$
\widehat{\sigma}_{w_{0}}:=\widehat{\psi}_{w_{0}}\left(\widehat{\Sigma}_{w_{0}}\right)=\widehat{\Sigma}_{w_{0}}\left(w_{0}\right)=\left\{g\left(w_{0}\right) \in M^{\prime}: g \in \widehat{\Sigma}_{w_{0}}\right\} \subset M^{\prime} .
$$

Since $\widehat{H}_{w_{0}}$ is a closed connected Lie subgroup of $G$, it follows from the inclusion $\widehat{H}_{w_{0}} \subset \widehat{\Sigma}_{w_{0}}$ that $\widehat{\sigma}_{w_{0}}$ is an irreducible, $m_{1}$-dimensional non-singular $f$-generalized analytic set in $M^{\prime}$ passing through $w_{0}$. Now to each tuple

$\left\{G ; M=G / H_{z_{0}} ; D \Subset M ; z_{0} \in D ; \mathfrak{X}_{z_{0}} \subset \mathfrak{X} ; \Sigma_{z_{0}} \subset D\left(z_{0}\right) \subset G ; \sigma_{z_{0}} \Subset D\right\}$ from Theorem 6.3 where $H_{z_{0}}$ is connected in $G$ (so that $H_{z_{0}} \subset \Sigma_{z_{0}}$ ) we assign a corresponding tuple

$$
\left\{G ; M^{\prime}=G / \widehat{H}_{w_{0}} ; \widehat{D}_{1} \subset M^{\prime} ; w_{0} \in \widehat{D}_{1} ; \widehat{\mathfrak{X}}_{w_{0}} \subset \mathfrak{X} ; \widehat{\Sigma}_{w_{0}} \subset D^{\prime}\left(z_{0}\right) \subset G ; \widehat{\sigma}_{w_{0}} \subset \widehat{D}_{1}\right\}
$$

from Theorem 6.4 where $\widehat{H}_{w_{0}}$ is connected in $G$ (so that $\widehat{H}_{w_{0}} \subset \widehat{\Sigma}_{w_{0}}$ ). Note that, in general, $\widehat{\sigma}_{w_{0}} \notin \widehat{D}_{1}$. Then by the same argument used to obtain the foliations of $M$ and $D$ in (6.21), we obtain the following foliations of $M^{\prime}$ and $\widehat{D}_{1}$

$$
M^{\prime}=\bigcup_{g \in G} g \widehat{\sigma}_{w_{0}} \quad \text { and } \quad \widehat{D}_{1}=\bigcup_{g \in D^{\prime}\left(z_{0}\right)} g \widehat{\sigma}_{w_{0}},
$$

despite the fact that $\widehat{D}_{1}$ is not necessarily relatively compact in $M^{\prime}$. Therefore, given $w \in M^{\prime}\left(\widehat{D}_{1}\right)$, we have a unique leaf $g \widehat{\sigma}_{w_{0}} \subset M^{\prime}\left(\widehat{D}_{1}\right)$ which passes through $w$. Here $g \in G\left(D^{\prime}\left(z_{0}\right)\right)$ is uniquely determined up to $\widehat{H}_{w_{0}}$. i.e., if another leaf $g^{\prime} \widehat{\sigma}_{w_{0}}$ passes through $w$, then $g^{\prime}=g h$ for some $h \in H^{\prime}\left(z_{0}\right)=\widehat{H}_{w_{0}}$.

With these preliminaries, we commence with the following lemma related to the representation of $D$ and $\partial D$ in (6.17): $D=\bigcup_{g \in D^{\prime}\left(z_{0}\right)} g \sigma_{z_{0}}$ and $\partial D=$ $\bigcup_{g \in \partial D^{\prime}\left(z_{0}\right)} g \sigma_{z_{0}}$. 


\section{Lemma 9.5.}

1. The set $\sigma_{z_{0}}$ in $M$ is an $m_{1}$-dimensional non-singular $f$-generalized analytic set in $M$ with $\sigma_{z_{0}} \Subset D$.

2. The union $\bigcup_{g \in D^{\prime}\left(z_{0}\right)} g \sigma_{z_{0}}$ is a foliation.

Proof. We verified that $\sigma_{z_{0}} \Subset D$ in (6.17). Since 1. follows from 2., we prove 2 . First we verify the following elementary equality:

$$
\text { for any } g \in D^{\prime}\left(z_{0}\right), \quad \widehat{\pi}^{-1}\left(g \sigma_{z_{0}}\right) \cap \widehat{D}_{1}=\bigcup_{k=1}^{\infty} g h_{k}^{\prime} \widehat{\sigma}_{w_{0}} \text {. }
$$

Indeed, letting $h_{k}^{\prime} \in \mathcal{H}^{\prime}\left(z_{0}\right)$, we have $h_{k}^{\prime} \widehat{\Sigma}_{w_{0}}=h_{k}^{\prime} \Sigma_{z_{0}} \subset D^{\prime}\left(z_{0}\right)=\widehat{D}_{1}\left(w_{0}\right)$ from 2 (ii). in Lemma 9.3 so that $h_{k}^{\prime} \widehat{\sigma}_{w_{0}}=h_{k}^{\prime} \widehat{\Sigma}_{w_{0}}\left(w_{0}\right) \subset \widehat{D}_{1}$. Using 1 . in Lemma 9.3 we obtain

$$
\widehat{\pi}\left(g h_{k}^{\prime} \widehat{\sigma}_{w_{0}}\right)=\widehat{\pi}\left(g h_{k}^{\prime} \widehat{\Sigma}_{w_{0}}\left(w_{0}\right)\right)=g h_{k}^{\prime} \Sigma_{z_{0}}\left(z_{0}\right)=g \Sigma_{z_{0}} h_{k}^{\prime}\left(z_{0}\right)=g \sigma_{z_{0}} .
$$

This proves that $\bigcup_{k=1}^{\infty} g h_{k}^{\prime} \widehat{\sigma}_{w_{0}} \subset \widehat{\pi}^{-1}\left(g \sigma_{z_{0}}\right) \cap \widehat{D}_{1}$. Conversely, let $w^{\prime} \in$ $\widehat{\pi}^{-1}\left(g \sigma_{z_{0}}\right) \cap \widehat{D}_{1}$. Using (9.4) we can find $g^{\prime} \in D^{\prime}\left(z_{0}\right)$ with $g^{\prime}\left(w_{0}\right)=w^{\prime}$. We thus have $g^{\prime}\left(z_{0}\right)=\widehat{\pi}\left(g^{\prime}\left(w_{0}\right)\right)=\widehat{\pi}\left(w^{\prime}\right) \in g \sigma_{z_{0}}=g \Sigma_{z_{0}}\left(z_{0}\right)$; hence we can find $s \in \Sigma_{z_{0}}$ and $h \in H_{z_{0}}$ with $g^{\prime}=g s h$ in $G$. From 2. (ii) in Lemma 9.3, $g s$ and $g^{\prime}$ are contained in $D^{\prime}\left(z_{0}\right)$. It follows from 3. in Propositon 6.1 that $h \in \mathcal{H}^{\prime}\left(z_{0}\right)$; hence there exists $h_{k}^{\prime}$ with $h \in h_{k}^{\prime} \widehat{H}_{w_{0}}$. Using 1 . in Lemma 9.3, we have

$$
w^{\prime}=g \operatorname{sh}\left(w_{0}\right) \in g \Sigma_{z_{0}} h\left(w_{0}\right)=g \Sigma_{z_{0}} h_{k}^{\prime}\left(w_{0}\right)=g h_{k}^{\prime} \Sigma_{z_{0}}\left(w_{0}\right)=g h_{k}^{\prime} \widehat{\sigma}_{w_{0}},
$$

proving the reverse inclusion.

Finally, we note that $\widehat{\pi}: M \rightarrow M^{\prime}$ is a normal covering and $\widehat{D}_{1}=$ $\bigcup_{g \in D^{\prime}\left(z_{0}\right)} g \widehat{\sigma}_{w_{0}}$ is a foliation of leaves of $m_{1}$-dimensional nonsingular generalized analytic sets $g \widehat{\sigma}_{w_{0}}$. It follows from formula (9.12) that $D=\bigcup_{g \in D^{\prime}\left(z_{0}\right)} g \sigma_{z_{0}}$ is a foliation of leaves of $m_{1}$-dimensional non-singular $f$-generalized analytic sets $g \sigma_{z_{0}}$; hence Lemma 9.5 is proved.

We construct a domain $K$ as in $2-a$ (i) of Theorem 6.4. in the following lemma.

Lemma 9.6. Let $D \Subset M$ and $f i x z_{0} \in D$ as above. Let $E$ be a domain with smooth boundary in $M$ such that $D \Subset E \subset M$ and $D$ and $E$ are of the same isotropy class at $z_{0}$ in $D$ (such $E$ exist by Lemma 9.2). Then there exists a domain $K$ in $M$ with $D \Subset K \subset E$ such that $K$ is foliated by $m_{1}$-dimensional non-singular $f$-generalized analytic sets $g \sigma_{z_{0}}$ in $M$ :

$$
K=\bigcup_{g \in K^{\prime}\left(z_{0}\right)} g \sigma_{z_{0}} \quad \text { with } g \sigma_{z_{0}} \Subset K .
$$


Proof. Fix a neighborhood $\mathcal{V}$ of $e$ in $G$ such that $f(\bar{D}) \Subset E$ for any $f \in \mathcal{V}$. We apply Lemma 9.1 for $D$ and $\mathcal{V}$ to obtain balls $V^{(i)}, i=1, \ldots, N$, centered at $z_{i} \in D$ in $M$ and $U_{k}^{(i)}$ in $M^{\prime}$ which satisfy conditions $(1) \sim(3)$ in the lemma as well as the additional condition that $V^{(i)} \Subset E, i=1, \ldots N$. We set $\widehat{\pi}^{-1}\left(z_{i}\right)=\left\{w_{k}^{(i)}\right\}_{k=1,2, \ldots} \in \widehat{\pi}^{-1}(D) \subset M^{\prime}$; i.e., $w_{k}^{(i)}$ is the center of $U_{k}^{(i)}$. We may assume that $A:=\cup_{i=1}^{N} V^{(i)}$ is a connected domain in $M$ with $D \Subset A \Subset E$. Since $E$ and $D$ are of the same isotropy class at $z_{0}$, so are $A$ and $D$; i.e., $D^{\prime}\left(z_{0}\right) \cap H_{z_{0}}=A^{\prime}\left(z_{0}\right) \cap H_{z_{0}}=E^{\prime}\left(z_{0}\right) \cap H_{z_{0}}$.

For each $i=1, \ldots, N$, we consider the sets $U_{k}^{(i)} \subset M^{\prime}$ defined in (2) in Lemma 9.1. We only consider the sets $U_{k}^{(i)}$ in $\left\{U_{k}^{(i)}\right\}_{k=1,2, \ldots}$ which are contained in $\widehat{E}_{1}$, the connected component of $\widehat{\pi}^{-1}(E)$ containing $w_{0}$ in $M^{\prime}$. By convention, we use the same notation $\left\{U_{k}^{(i)}\right\}_{i, k}$ for these sets. From (ii) in Lemma 9.2, $\widehat{A}_{1}=\widehat{E}_{1} \cap \widehat{\pi}^{-1}(A)$; thus the connected component $\widehat{A}_{1}$ of $\widehat{\pi}^{-1}(A)$ in $M^{\prime}$ containing $w_{0}$ may be decomposed as

$$
\widehat{A}_{1}=\bigcup_{i, k} U_{k}^{(i)}, \quad i=1, \ldots, N ; k=1,2, \ldots
$$

We claim that

$$
K:=\bigcup_{g \in A^{\prime}\left(z_{0}\right)} g \sigma_{z_{0}},
$$

satisfies the conclusion of Lemma 9.6. Note that $A^{\prime}\left(z_{0}\right)$ and $A$ in $M$ are domains in $G$ and $\sigma_{z_{0}}$ is connected in $M$; thus $K$ is a domain in $M$.

We begin by showing that

$$
K=\bigcup_{g \in K^{\prime}\left(z_{0}\right)} g \sigma_{z_{0}} \quad \text { with } \quad g \sigma_{z_{0}} \Subset K .
$$

We first show $A \subset K \subset E$. Indeed, $A \subset K$ follows from $A=\left\{g\left(z_{0}\right) \in M\right.$ : $\left.g \in A^{\prime}\left(z_{0}\right)\right\}$ and $z_{0} \in \sigma_{z_{0}}$. To prove $K \subset E$, we first show that

$$
\sigma_{k}^{(i)}(w) \sigma_{z_{0}} \Subset E, \quad w \in U_{k}^{(i)} ; i=1, \ldots, N ; k=1,2, \ldots,
$$

where $\sigma_{k}^{(i)}$ is the holomorphic section of $G$ over $U_{k}^{(i)}$ via $\widehat{\psi}_{w_{0}}$ defined in (3) in Lemma 9.1.

Since $\widehat{\pi}\left(w_{k}^{(i)}\right)=z_{i} \in D$, we see from (ii) in Lemma 9.2 that $\sigma_{k}^{(i)}\left(w_{k}^{(i)}\right)\left(w_{0}\right)=$ $w_{k}^{(i)} \in U_{k}^{(i)} \subset \widehat{E}_{1} \cap \widehat{\pi}^{-1}(D)=\widehat{D}_{1}$, so that $\sigma_{k}^{(i)}\left(w_{k}^{(i)}\right) \in \widehat{D}_{1}\left(w_{0}\right)=D^{\prime}\left(z_{0}\right)$ by (9.4). It follows from 2. in Lemma 9.5 that $\sigma_{k}^{(i)}\left(w_{k}^{(i)}\right) \sigma_{z_{0}} \Subset D$. Now let $w \in U_{k}^{(i)}$, and set $f:=\left[\sigma_{k}^{(i)}(w)\right]\left[\sigma_{k}^{(i)}\left(w_{k}\right)\right]^{-1} \in G$. Then by (3) in Lemma 9.1 we have $f \in \mathcal{V}$. It follows that $\sigma_{k}^{(i)}(w) \sigma_{z_{0}}=f\left[\sigma_{k}^{(i)}\left(w_{k}^{(i)}\right)\right] \sigma_{z_{0}} \subset f(D) \Subset E$, as required.

Next we show that $g \sigma_{z_{0}} \Subset E$ for any $g \in A^{\prime}\left(z_{0}\right)$. Let $g \in A^{\prime}\left(z_{0}\right)$. Since $g\left(w_{0}\right) \in \widehat{A}_{1}$, we have $w:=g\left(w_{0}\right) \in U_{k}^{(i)}$ for some $i, k$. Since $\sigma_{k}^{(i)}(w)\left(w_{0}\right)=$ 
$w=g\left(w_{0}\right)$, we can find an $h \in \widehat{H}_{w_{0}}$ such that $g=\sigma_{k}^{(i)}(w) h$. Since $h \sigma_{z_{0}}=\sigma_{z_{0}}$, we have $g \sigma_{z_{0}}=\left(\sigma_{k}^{(i)}(w) h\right) \sigma_{z_{0}}=\sigma_{k}^{(i)}(w) \sigma_{z_{0}} \Subset E$, as required.

Now we show that $K=\bigcup_{g \in K^{\prime}\left(z_{0}\right)} g \sigma_{z_{0}}$. Since $A \subset K$, we have the inclusion $K \subset \bigcup_{g \in K^{\prime}\left(z_{0}\right)} g \sigma_{z_{0}}$. To prove the reverse inclusion, let $g^{\prime} \in K^{\prime}\left(z_{0}\right)$. Then $g^{\prime}\left(z_{0}\right) \in K$, so that we can find $g \in A^{\prime}\left(z_{0}\right)$ and $s \in \Sigma_{z_{0}}$ with $g^{\prime}\left(z_{0}\right)=$ $g s\left(z_{0}\right)$. Thus there exists $h \in H_{z_{0}}$ with $g^{\prime}=g s h$ in $G$. We note that $g s \in K^{\prime}\left(z_{0}\right)$. To see this, since $\Sigma_{z_{0}}$ is connected and contains $e$ in $G$, there exists a continuous curve $\gamma: t \in[0,1] \rightarrow s(t)$ in $\Sigma_{z_{0}}$ with $s(0)=e$ and $s(1)=s$. The continuous curve $\widetilde{\gamma}: t \in[0,1] \rightarrow \widetilde{\gamma}(t):=g s(t)$ in $G$ satisfies $\widetilde{\gamma}(t)\left(z_{0}\right) \in g \sigma_{z_{0}} \subset K$. Since $\widetilde{\gamma}(0)=g \in A^{\prime}\left(z_{0}\right) \subset K^{\prime}\left(z_{0}\right)$, we have $\widetilde{\gamma} \subset K^{\prime}\left(z_{0}\right)$, and hence $g s=\widetilde{\gamma}(1) \in K^{\prime}\left(z_{0}\right)$.

It follows from 2. in Proposition 6.1 applied to $g^{\prime}=g s h \in K^{\prime}\left(z_{0}\right)$ that $h \in K^{\prime}\left(z_{0}\right) \cap H_{z_{0}}=E^{\prime}\left(z_{0}\right) \cap H_{z_{0}}=\mathcal{H}^{\prime}\left(z_{0}\right)$. Consequently,

$$
\begin{aligned}
g^{\prime} \sigma_{z_{0}} & =g \operatorname{sh} \sigma_{z_{0}}=g \operatorname{sh} \Sigma_{z_{0}}\left(z_{0}\right) \\
& =g s \Sigma_{z_{0}} h\left(z_{0}\right) \quad(\text { by } 1 . \text { in Lemma 9.3) } \\
& =g \Sigma_{z_{0}}\left(z_{0}\right) \quad\left(\text { since } \Sigma_{z_{0}} \text { is a group and } h\left(z_{0}\right)=z_{0}\right) \\
& =g \sigma_{z_{0}} \subset K,
\end{aligned}
$$

as claimed.

To prove that $g \sigma_{z_{0}} \Subset K$ for a given $g \in K\left(z^{\prime}\right)$, since $K$ is open it suffices to show that for a given $\zeta \in \partial \sigma_{z_{0}} \Subset D$ we have $g(\zeta) \in K$. Since $K^{\prime}\left(z_{0}\right)$ is an open set in $G$ containing $e$, there is a ball $B$ centered at $e$ with $g B \Subset$ $K^{\prime}\left(z_{0}\right)$. From the previous assertion we have $g B \sigma_{z_{0}} \subset K$. It thus suffices to show $g(\zeta) \in g B \sigma_{z_{0}}$, or equivalently, $\zeta \in B \sigma_{z_{0}}$. This is also equivalent to $B^{-1}(\zeta) \cap \sigma_{z_{0}} \neq \emptyset$. This is clear, since $\zeta \in \partial \sigma_{z_{0}}$ and $B^{-1}(\zeta)$ is a neighborhood of $\zeta$ in $M$.

Finally, we shall show that the union $K=\bigcup_{g \in K^{\prime}\left(z_{0}\right)} g \sigma_{z_{0}}$ is a foliation. Indeed, using a similar argument as in the proof of (9.12), under the hypothesis $K^{\prime}\left(z_{0}\right) \cap H_{z_{0}}=\mathcal{H}^{\prime}\left(z_{0}\right)$, we have the following fact:

$$
\widehat{\pi}^{-1}\left(g \sigma_{z_{0}}\right) \cap \widehat{K}_{1}=\bigcup_{k=1}^{\infty} g h_{k}^{\prime} \widehat{\sigma}_{w_{0}} \quad \text { for } g \in K^{\prime}\left(z_{0}\right) .
$$

Moreover, since $\widehat{\pi}: M \rightarrow M^{\prime}$ is a normal covering and $\widehat{K}_{1}=\cup_{g \in K^{\prime}\left(z_{0}\right)} g \widehat{\sigma}_{w_{0}}$ is a foliation of leaves of $m_{1}$-dimensional nonsingular generalized analytic sets $g \widehat{\sigma}_{w_{0}}$, it follows from the above fact that $K=\cup_{g \in K^{\prime}\left(z_{0}\right)} g \sigma_{z_{0}}$ is a foliation of leaves of $g \sigma_{z_{0}}$, and hence Lemma 9.6 is proved.

The domain $K$ in $M$ as defined in (9.14) completes the proof of items (o) and (i) in $2-a$ of Theorem 6.4. For item (ii), let $\sigma \Subset D$ be a parabolic nonsingular generalized analytic set in $D$. Then we have $\lambda(z)=$ const. on $\sigma$. 
Since $D=\bigcup_{g \in D^{\prime}\left(z_{0}\right)} g \sigma_{z_{0}}$ is a foliation, we use the same argument as in the proof of (ii) in $1-a$ of Theorem 6.3 to obtain $\sigma \subset g \sigma_{z_{0}}$ for some $g \in D^{\prime}\left(z_{0}\right)$, as required.

To prove $2-b$. we assume that $\sigma_{z_{0}}$ is closed in $M$. Since $\sigma_{z_{0}} \Subset D, \sigma_{z_{0}}$ is an $m_{1}$-dimensional, irreducible, nonsingular, compact analytic set in $D$. The outline of the proof of $2-b$. is as follows. First, using (i) in $2-a$, we may consider the quotient space $K_{0}:=K / \sigma_{z_{0}}$ which is an $\left(n-m_{1}\right)$-dimensional connected complex manifold. Moreover, $D_{0}:=D / \sigma_{z_{0}}$ is a subdomain of $K_{0}$, and $\partial D_{0}$ is smooth in $K_{0}$. This last fact follows from (6.17): $\partial D=$ $\cup_{g \in \partial D^{\prime}\left(z_{0}\right)} g \sigma_{z_{0}}$, together with the smoothness of $\partial D$.

To be precise, we work in the Lie group $G$. We use the notation $G_{1} \approx G_{2}$ to mean that $G_{1}$ and $G_{2}$ are isomorphic as complex manifolds. Since $\mathcal{H}^{\prime}\left(z_{0}\right)$ and $\Sigma_{z_{0}} \mathcal{H}^{\prime}\left(z_{0}\right)$ are closed Lie subgroups of $G$ by 2. in Lemma 9.3 , we may consider the quotient spaces

$$
\mathfrak{A}:=G / \mathcal{H}^{\prime}\left(z_{0}\right) \quad \text { and } \quad \mathfrak{B}:=G / \Sigma_{z_{0}} \mathcal{H}^{\prime}\left(z_{0}\right) ;
$$

then $\mathfrak{A}$ is an $n$-dimensional connected complex manifold and $\mathfrak{B}$ is an $\left(n-m_{1}\right)$ dimensional connected complex manifold. Since $\mathcal{H}^{\prime}\left(z_{0}\right) \subset \Sigma_{z_{0}} \mathcal{H}^{\prime}\left(z_{0}\right)$ we have the canonical projection:

$$
\pi_{b}^{a}: g \mathcal{H}^{\prime}\left(z_{0}\right) \in \mathfrak{A} \mapsto g \Sigma_{z_{0}} \mathcal{H}^{\prime}\left(z_{0}\right) \in \mathfrak{B},
$$

and the quotient space

$$
\mathfrak{C}:=\Sigma_{z_{0}} \mathcal{H}^{\prime}\left(z_{0}\right) / \mathcal{H}^{\prime}\left(z_{0}\right) \quad \text { with }\left(\pi_{b}^{a}\right)^{-1}(\zeta) \approx \mathfrak{C} \text { for } \zeta \in B
$$

From 2. (iv) in Lemma 9.3 we have $\mathfrak{C} \approx \sigma_{z_{0}}$.

Since $D^{\prime}\left(z_{0}\right) \mathcal{H}^{\prime}\left(z_{0}\right)=D^{\prime}\left(z_{0}\right)$ and $K^{\prime}\left(z_{0}\right) \mathcal{H}^{\prime}\left(z_{0}\right)=K^{\prime}\left(z_{0}\right)$, the quotients $D^{\prime}\left(z_{0}\right) / \mathcal{H}^{\prime}\left(z_{0}\right)$ and $K^{\prime}\left(z_{0}\right) / \mathcal{H}^{\prime}\left(z_{0}\right)$ are well-defined and define domains, say $D_{a}$ and $K_{a}$, in $\mathfrak{A}$. Since $\left(\partial D^{\prime}\left(z_{0}\right)\right) \mathcal{H}^{\prime}\left(z_{0}\right)=\partial D^{\prime}\left(z_{0}\right)$, we have $D_{a} \Subset K_{a}$ and $\partial D_{a}$ is smooth in $K_{a}$. Similarly, since $D^{\prime}\left(z_{0}\right) \Sigma_{z_{0}} \mathcal{H}^{\prime}\left(z_{0}\right)=D^{\prime}\left(z_{0}\right)$ and $K^{\prime}\left(z_{0}\right) \Sigma_{z_{0}} \mathcal{H}^{\prime}\left(z_{0}\right)=K^{\prime}\left(z_{0}\right)$ by 2. (ii) in Lemma 9.3, the quotients $D^{\prime}\left(z_{0}\right) / \Sigma_{z_{0}} \mathcal{H}^{\prime}\left(z_{0}\right)$ and $K^{\prime}\left(z_{0}\right) / \Sigma_{z_{0}} \mathcal{H}^{\prime}\left(z_{0}\right)$ define domains, say $D_{0}$ and $K_{0}$, in $\mathfrak{B}$. Since $g \in$ $\partial D^{\prime}\left(z_{0}\right)$ implies $g \Sigma_{z_{0}} \mathcal{H}^{\prime}\left(z_{0}\right) \subset \partial D^{\prime}\left(z_{0}\right)$, it follows that $D_{0} \Subset K_{0}$ and $\partial D_{0}$ is smooth in $K_{0}$. Thus, $\pi_{b}^{a}\left(D_{a}\right)=D_{0}$ and $\pi_{b}^{a}\left(K_{a}\right)=K_{0}$.

On the other hand, we have $D_{a} \approx D$ and $K_{a} \approx K$. To see this, since $\mathcal{H}^{\prime}\left(z_{0}\right) \subset H_{z_{0}}$, we have the canonical projection

$$
\pi_{1}: g \mathcal{H}^{\prime}\left(z_{0}\right) \in \mathfrak{A} \mapsto g H_{z_{0}}=g\left(z_{0}\right) \in M,
$$

so that $\left(\mathfrak{A}, \pi_{1}\right)$ is an unramified covering of $M$ (since $\widehat{H}_{w_{0}} \subset \mathcal{H}^{\prime}\left(z_{0}\right) \subset H_{z_{0}}$ and $\widehat{H}_{w_{0}}$ is a normal subgroup of $\left.H_{z_{0}}\right)$. Then the restriction $\pi_{1}: D_{a}\left(K_{a}\right) \mapsto$ 
$D(K)$ is bijective. We only give the proof for $\pi_{1}: K_{a} \mapsto K$. The surjectivity comes from 1. in Proposition 6.1 (applied to $K$ instead of $D$ ). To prove the injectivity, let $g_{1}, g_{2} \in K^{\prime}\left(z_{0}\right)$ with $g_{1}\left(z_{0}\right)=g_{2}\left(z_{0}\right)$. We can find $h \in H_{z_{0}}$ with $g_{1}=g_{2} h$. It follows from 3. in Proposition 6.1 that $h \in H_{z_{0}} \cap K^{\prime}\left(z_{0}\right)=$ $\mathcal{H}^{\prime}\left(z_{0}\right)$. Since $\mathcal{H}^{\prime}\left(z_{0}\right)$ is a group, we have $g_{1} \mathcal{H}^{\prime}\left(z_{0}\right)=g_{2} h \mathcal{H}^{\prime}\left(z_{0}\right)=g_{2} \mathcal{H}^{\prime}\left(z_{0}\right)$, as required.

Setting $\pi_{0}:=\left.\left.\pi_{b}^{a}\right|_{K_{A}} \circ\left(\pi_{1}\right)^{-1}\right|_{K}$, we have the holomorphic surjection

$$
\pi_{0}: K \mapsto K_{a} \mapsto K_{0}
$$

which satisfies $\pi_{0}(D)=D_{0}$. Moreover, let $z_{1}, z_{2} \in K$ and let $g_{1}, g_{2} \in K^{\prime}\left(z_{0}\right)$ with $z_{1}=g_{1}\left(z_{0}\right)$ and $z_{2}=g_{2}\left(z_{0}\right)$. Then

$$
\pi_{0}\left(z_{1}\right)=\pi_{0}\left(z_{2}\right) \quad \text { iff } \quad g_{1} \sigma_{z_{0}}=g_{2} \sigma_{z_{0}} .
$$

Assume first that $\pi_{0}\left(z_{1}\right)=\pi_{0}\left(z_{2}\right)$. Then by the definition of $\pi_{0}$ we have $g_{1} \Sigma_{z_{0}} \mathcal{H}^{\prime}\left(z_{0}\right)=g_{2} \Sigma_{z_{0}} \mathcal{H}^{\prime}\left(z_{0}\right)$. Since $g_{i} \Sigma_{z_{0}} \mathcal{H}^{\prime}\left(z_{0}\right)\left(z_{0}\right)=g_{i} \sigma_{z_{0}}, \quad i=1$, 2, we have $g_{1} \sigma_{z_{0}}=g_{2} \sigma_{z_{0}}$. Conversely, assume that $g_{1} \sigma_{z_{0}}=g_{2} \sigma_{z_{0}}$. Then we have $s_{1}, s_{2} \in \Sigma_{z_{0}}$ and $h \in H_{z_{0}}$ with $g_{1} s_{1}=g_{2} s_{2} h$. By (3) in Lemma 9.3 (replacing $D^{\prime}\left(z_{0}\right)$ by $\left.K^{\prime}\left(z_{0}\right)\right)$ we have $g_{i} s \in K^{\prime}\left(z_{0}\right), i=1,2$. It follows from 3 . in Proposition 6.1 that $h \in K^{\prime}\left(z_{0}\right) \cap H_{z_{0}}=\mathcal{H}^{\prime}\left(z_{0}\right)$. Since $\Sigma_{z_{0}} \mathcal{H}^{\prime}\left(z_{0}\right)$ is a group, we have $g_{1} \Sigma_{z_{0}} \mathcal{H}^{\prime}\left(z_{0}\right)=g_{2} \Sigma_{z_{0}} \mathcal{H}^{\prime}\left(z_{0}\right)$; i.e., $\pi_{0}\left(\zeta_{1}\right)=\pi_{0}\left(\zeta_{2}\right)$. Thus (9.16) is proved.

In particular, (9.16) implies that $\pi_{0}^{-1}(\zeta) \approx \sigma_{z_{0}}$ for each $\zeta \in K$, and $\pi_{0}^{-1}\left(D_{0}\right)=\cup_{g \in D^{\prime}\left(z_{0}\right)} g \sigma_{z_{0}}=D$.

To complete the proof of $2-b$. in Theorem 6.4, it remains to verify that $D_{0}$ is a Stein domain in $K_{0}$.

Fix $\zeta \in D_{0}$. Take a point $z \in D$ with $\pi_{0}(z)=\zeta$ and define $\lambda_{0}(\zeta):=$ $\lambda(z)$. If we take another point $z^{\prime} \in D$ with $\pi_{0}\left(z^{\prime}\right)=\zeta$, there exists $g \in$ $D^{\prime}\left(z_{0}\right)$ with $z=g\left(z_{0}\right)$, and hence $z^{\prime} \in g \sigma_{z_{0}}$ by (9.16). Since $\lambda\left(g \sigma_{z_{0}}\right)=$ const. $=\lambda\left(g\left(z_{0}\right)\right)=\lambda(z)$, it follows that $\lambda\left(z^{\prime}\right)=\lambda(z)$; thus $\lambda_{0}$ is a welldefined real-valued function on $D_{0}$. Since $\pi_{0}(D)=D_{0}$, it follows that $-\lambda_{0}$ is plurisubharmonic exhaustion function on $D_{0}$. Noting that assertion $\alpha$. in the proof of Theorem 6.3 holds under the conditions in Theorem 6.4, using the same method as in the proof of $1-b$. of Theorem 6.3, we see that $-\lambda_{0}$ is strictly plurisubharmonic on $D_{0}$; i.e., $D_{0}$ is Stein.

\section{References}

[1] K. Adachi, Le problème de Lévi pour les fibrés grassmanniens et les variétés de drapeaux, Pacific J. Mem. 116 (1985), 1-6. 
[2] L.V. Ahlfors and L. Sario, Riemann Surfaces, Princeton, New Jersey 1960 .

[3] A. Hirshowitz, Le problème de Levi pour les espaces homogenes, Bull. Soc. Math. France, 103 (1975), 191-201.

[4] K. Diederich and T. Ohsawa, A Levi problem on two-dimensional complex manifolds, Math. Ann., 261 (1982), 255-261.

[5] H. Grauert, Bemerkenswerte pseudokonvexe Mannigfaltigkeiten, Math. Zeitschr., 81 (1963), 377-391.

[6] J-C. Joo, On the Levenberg-Yamaguchi formula for the Robin function, to appear.

[7] H. Kazama, On pseudoconvexity of complex Lie groups, Mem. Fac. Sci. Kyushu Univ. 27 (1973), 241-247.

[8] H. Kazama, D. K. Kim and C. Y. Oh, Some remarks on complex Lie groups, Nagoya Math. J., 157 (2000), 47-57.

[9] N. Levenberg and H. Yamaguchi, The metric induced by the Robin function, Memoirs of the A. M. S. vol. 92 \#448 (1991), 1-156.

[10] K-T. Kim, N. Levenberg and H. Yamaguchi, Robin functions for complex manifolds and applications, to appear in 2007 Proceedings of the Kiselmanfest.

[11] N. Levenberg and H. Yamaguchi, Robin functions for complex manifolds and applications, CR Geometry and Isolated Singularities, Report of RIMS of Kyoto Univ. 1037, 138-142, 1998.

[12] D. Michel, Sur les ouverts pseudoconvex des espaces homogénes, C.R.Acad., Sci. Paris, Sér. A, Math., 283(1976), 779-783.

[13] M. Nakai and L. Sario, Classification Theory of Riemann Surfaces, Springer-Verlag, New York 1970.

[14] Y-T. Siu, Pseudoconvexity and the problem of Levi, Bulletin of AMS, 84 (1978), 481-512.

[15] T. Nishino, Function Theory in Several Complex Variables, Mathematical Monographs of the A.M.S. vol. 193, 2001.

[16] T. Ohsawa, On the Levi-flats in complex tori of dimension two, Publ. RIMS, 42 (2006), 361-377. Supplement, 379-382. 
[17] Z. Slodkowski and G. Tomassini, Minimal kernels of weakly complete spaces, J. of Functional Analysis, 210 (2004), 125-147.

[18] O. Suzuki, Remarks on examples of 2-dimensional weakly 1-complete manifolds which admit only constant holomorphic functions, J. Fac. Sci. Univ. Tokyo, Sect. IA Math. 25 (1979), no. 3, 253-261.

[19] T. Ueda, Pseudoconvex domains over Grassmann manifolds, J. of Math. Kyoto Univ., 20 (1980), 391-394.

[20] H. Yamaguchi, Variations of pseudoconvex domains over $\mathbf{C}^{n}$, Mich. Math. J. 36 (1989), 415-457. 